\title{
THE USE OF NANOSTRUCTURED CALCIUM SILICATE IN SOLAR CELLS
}

MASTERS THESIS

SUPERVISED BY:

DR THOMAS BORRMANN

DR MICHAEL RICHARDSON

SCHOOL OF CHEMICAL AND PHYSICAL SCIENCES 


\section{ABSTRACT}

Nanostructured calcium silicate (NCaSil) had previously been found to be photoactive and mildly semiconducting. Its use in solar cells was investigated in this project. Many different types of solar cells exist. Most common on the market are silicon-based cells, which generate charge separation through electric fields at $p / n$ junctions. Over the last decade, dye-sensitised solar cells (DSSCs) have been heavily researched. DSSCs depend on effective electron/hole separation at the dye and efficient transfer to the electron- and hole-conducting materials. An older and little-researched form of cells is the photogalvanic cell, of which there are two forms. One contains a semiconducting material, whereas the other comprises of either one or two redox couples, in which at least one species is photoactive. An example of the latter form of cell is the iodide/triiodide redox couple, which is commonly the electrolyte of choice in DSSCs and semiconductor-containing photogalvanic cells.

This project predominantly investigated the use of NCaSil in conjunction with the iodide/triiodide redox couple and its use in solar cells. The project ascertained that, when used with the iodide/triiodide, the NCaSil did not act as a semiconducting material (either as in a DSSC or semiconductor photogalvanic cell). Rather iodide/triiodide's photogalvanic process dominated the cell, despite the presence of NCaSil. Furthermore, the addition of the stable NCaSils to the iodide/triiodide (with $5 \mathrm{wt} \% \mathrm{CaCl}_{2}$ ) created "soggy sand electrolytes". These electrolytes showed increased conductivities, despite their higher viscosities, due to a synergistic effect. Soggy sand electrolytes show great promise in the development of more solid-like DSSCs.

Furthermore, the project observed that the performance of NCaSil cells was maximized with a 70 wt $\%$ ethanol (30 wt $\%$ water) solvated electrolyte, with $1.5 \mathrm{wt} \% \mathrm{CaCl}_{2}$ added to this electrolyte (or 5 wt $\% \mathrm{CaCl}_{2}$ in the water content). When used long-term in conjunction with Reinforced NCaSil, a gel was formed, which showed promising activity. This activity was attributed to the interaction of surface-bound $\mathrm{Ca}^{2+}$ to iodine. Similar gels formed from vanadium- and cerium-treated NCaSil also showed great cell performance. Cell performance was further enhanced by backing the cell with a reflective or light scattering material, such as Teflon tape.

Acknowledgements: William Georgetti Scholarship, Victoria Masters by Thesis Scholarship, Curtis Gordon Scholarship; Drs Thomas Borrmann and Michael Richardson; Michelle Lai, Teresa Vaughan and Sean O’Connor. 
LIST OF FIGURES ….................................................................

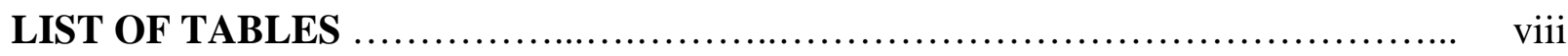

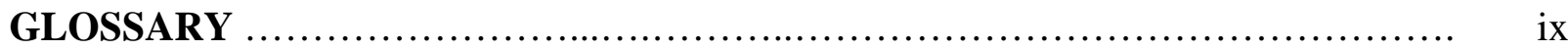

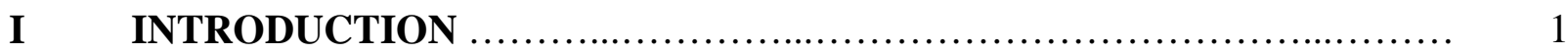

1 Dye-sensitised Solar Cells (DSSCs) .................................... 1

2 Solid-state DSSCs .......................................................... 25

$3 \quad$ Photogalvanic Solar Cells ............................................... 26

$4 \quad$ Nanostructured Calcium Silicate ........................................... $\quad 30$

$5 \quad$ Measuring Cell Performance …...................................... 35

II RESEARCH OBJECTIVES .............................................. 37

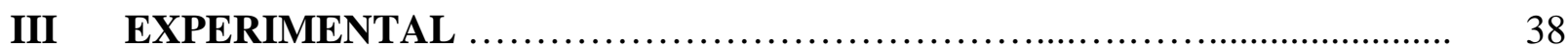

1 Synthesis and Development of Nanostructured Calcium Silicate ........... 38

2 Electrolytes Used ............................................................ 41

3 Synthesis of Tetraethoxyorthosilicate-Derived Gels .............................. 43

$4 \quad$ Sodium Silicate and Ethylene Glycol Gels ................................... 43

$5 \quad$ Gasket Cell Fabrication ................................................ 43

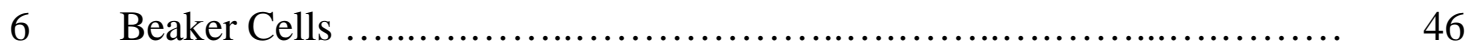

$7 \quad$ Quartz Cuvette Cells ................................................... 47

$8 \quad$ Testing the Effect of Cell Width and Depth .................................. 48

$9 \quad$ Stirring and Non-Stirring .................................................. 50

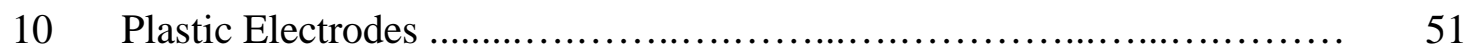

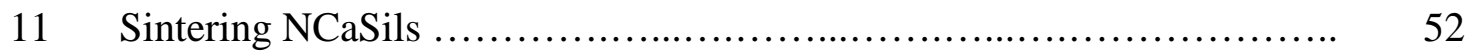

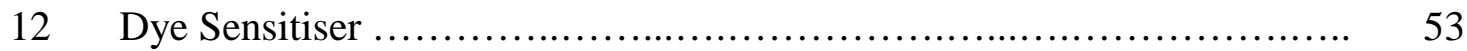

13 Light Source Used ............................................... 54

14 Cell Performance and Analysis ........................................... 54

15 Optical Properties .................................................... 55

16 Optical Density Filters ................................................... 55

17 Surface Area Measurements .................................................. 55

$18 \quad$ Particle Size Analysis ................................................. 56

19 Viscosity Measurements .............................................. 56

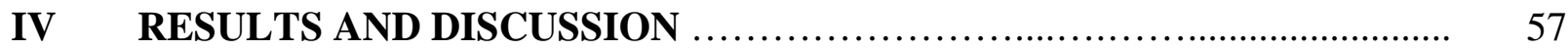

1 Different Forms of Nanostructured Calcium Silicate ...................... 57 


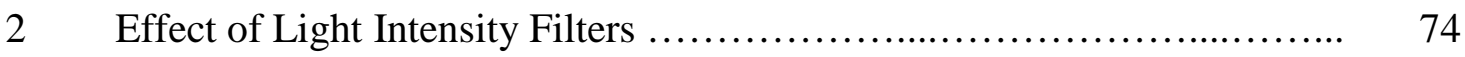

3 The Importance of the Silicate Backbone ............................ 77

$4 \quad$ The Electrolyte .......................................................... 82

$5 \quad$ Effect of Cell Surface Area and Sample Depth ........................ 100

$6 \quad$ Back-Reflection ....................................................... 110

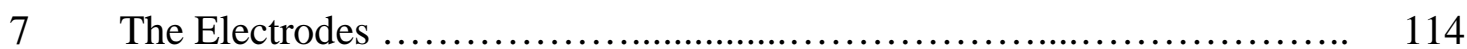

$8 \quad$ Long-Term Testing and Gel Formation ............................... 127

9 Performance and Analysis of NCaSil Gels .......................... 135

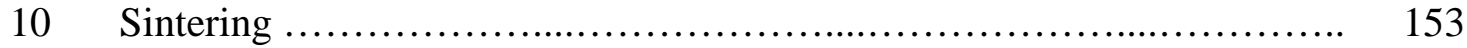

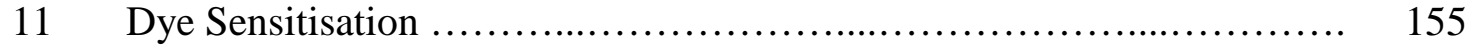

12 Conclusions on "Soggy Sand" Electrolytes ........................... 157

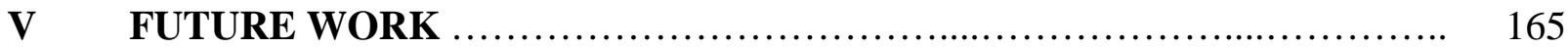

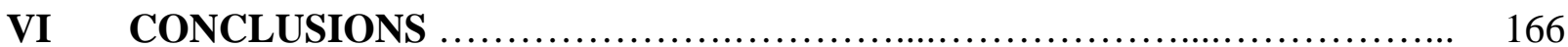

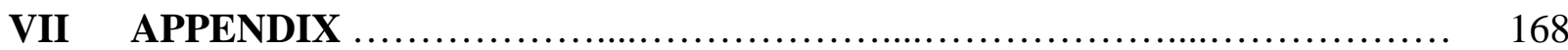

$1 \quad$ IR Spectra of RNCaSil and Differently Solvated Iodide/Triiodide ........ 168

2 UV-vis Transmission Spectrum of Laminated Plastic and ITO PET ...... 169

$3 \quad{ }^{1} \mathrm{H}$ and ${ }^{13} \mathrm{C}$ NMR Spectra of g-NCaSil .............................. 170

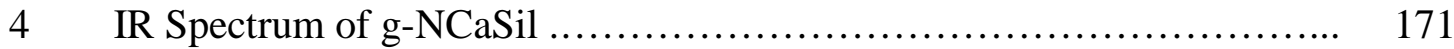

$5 \quad$ Gas IR Spectrum of Gas from TEOS Gel Formation .................... 171

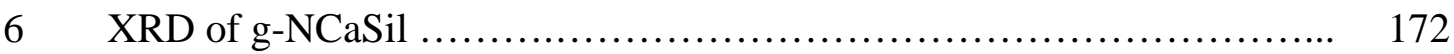

$7 \quad$ Solubility of $\mathrm{Ca}^{2+}$ at Different $\mathrm{pHs}$................................ 173

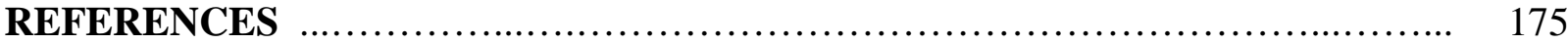




\section{LIST OF FIGURES}

Figure 1 Generalised Electron and Hole Orbital and Cell Movement in DSSCs ......... 3

Figure $2 \quad$ N719 and Black Dye used by Graetzel et al. .............................. 6

Figure 3 Spectral Absorbance Ranges of the N3 and Black Dye ................... 7

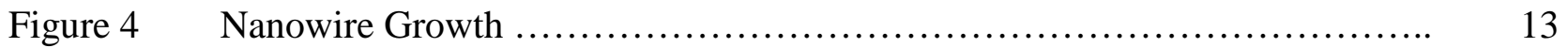

Figure 5 Effect of Cation on the Interfacial Energy of a Sensitised Electron-conducting Material ................................................................ 17

Figure $6 \quad p$-type Semiconductor Photogalvanic Cell ........................... 27

Figure $7 \quad$ Thin-Layer-Electrolyte Photogalvanic Cell f............................ 28

Figure $8 \quad$ Thin-Layer-Electrolyte Iodide/Triiodide Photogalvanic Cell ................ 29

Figure 9 Reinforced Nanostructured Calcium Silicate (at $\times 50000$ magnification ........ 30

Figure $10 \quad$ Absorbance of Dry and Wet Nanostructured Calcium Silicate ................ 31

Figure 11 Current versus Voltage Plots for a (a) Graetzel Type DSSC and (b)

Photogalvanic Cell ................................................. 35

Figure 12 Continuous Method Set Up ...................................... 40

Figure 13 "Gasket Cell" ................................................ 44

Figure $14 \quad$ "Beaker Cell" and Research Arc Lamp ............................... 46

Figure 15 Different Electrode Positions Tested (Birds-Eye View of Beaker Cell) .......... 47

Figure 16 Quartz Cuvette Testing: ITO Glass Front Electrode and Either a Pt-Coated or Pt-Wire Back Electrode .............................................. 48

Figure 17 Quartz Cuvette Testing: ITO Glass Back Electrode and a Pt-Wire Front Electrode, with or without ITO Glass in Front of the Cell .................. 48

Figure 18 Side Panel of the Slotted Vessel used to Test the Effect of Electrode Distance 49

Figure 19 Slotted Vessel used to Test the Effect of Electrode Distance ............... 50

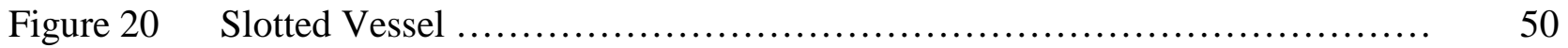

Figure $21 \quad$ Overhead Stirring of a Beaker Cell f.................................. 51

Figure 22 Representation of Laminated ITO-PET Cells ......................... 52

Figure 23 Tris(2,2'-bipyridyl)dichloro-ruthenium (II) hexahydrate ................... 53

Figure 24 Circuit Diagram of Set Up Used to Measure Cell Performance ............... 54

Figure 25 Different NCaSils with 0 or $5 \mathrm{wt} \% \mathrm{CaCl}_{2}$ in a water Iodide/Triiodide

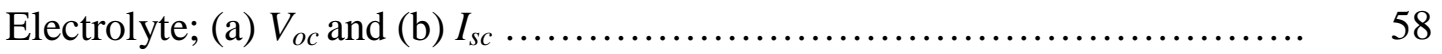

Figure 26 Current versus Voltage of Reinforced NCaSil in a Water Iodide/Triiodide Electrolyte with $5 \mathrm{wt} \% \mathrm{CaCl}_{2}\left(0.10 \mathrm{~g}: 1.0 \mathrm{~cm}^{3}\right)$ and of The Same Electrolyte Alone ............................................................................................ 60

Figure 27 Effect of Light Filters on the Reinforced Calcium Silicate Cell in Ethanol 
Iodide/Triiodide: (a) $V_{o c}$ and $I_{s c}$; (b) Power

Figure 28 UV-vis of the Water-Based and 70\% Ethanol $30 \%$ Water Iodide/Triiodide

Electrolytes, and RNCaSil with $100 \%$ Water-Based Iodide/Triiodide

Electrolyte

Figure 29 Cyclic Voltammetry of: (a) Water Iodide/Triiodide Electrolyte with Calcium

Chloride and NCaSils; and (b) $\mathrm{I}_{2}$-EENCaSil

Figure $30 \quad \mathrm{~V}$ - and Ce-Treated NCaSil; (a) $V_{o c}$ and (b) $I_{s c}$

Figure 31 Different RNCaSil Batches. Tested in a Normal Gasket with a C-coated Back

Electrode; $0.10 \mathrm{~g}$ RNCaSil: $1.0 \mathrm{~cm}^{3}$ Water Iodide/Triiodide (5 $\mathrm{wt} \% \mathrm{CaCl}_{2}$ )

Electrolyte; a) $V_{o c}$ and (b) $I_{s c}$

Figure 32 Particle Size Distribution Analysis of Three RNCaSils (Filter Cakes)

Figure 33 CM-RNCaSil in Water Iodide/Triiodide Electrolyte, with and without 5 wt\%

$\mathrm{CaCl}_{2}$; (a) $V_{o c}$ and (b) $I_{s c}$

Figure 34 Particle Size Distribution Analysis of Three Different Areas of the Same

Batch of CM-RNCaSil (Powder)

Figure 35 Effect of Light Intensity of the Cell Performance of RNCaSil in $70 \%$ Ethanol

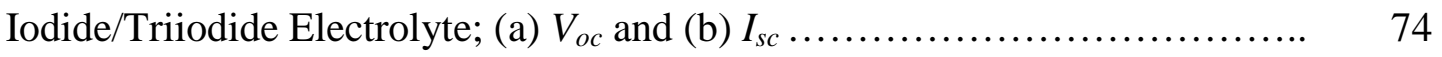

Figure $36 \quad$ UV-vis of the Optical Density Filters ................................ 75

Figure 37 Irradiance from the Ozone-Free, Xenon-Arc Discharge Lamp Used .......... 76

Figure 38 Spectral Irradiance from the Sun .................................. 76

Figure 39 Current versus Voltage of $\mathrm{CaI}_{2}$ in a Gasket Cell ......................... 78

Figure $40 \quad \mathrm{UV}$-vis of $\mathrm{CaI}_{2}$ in Water Compared to the Iodide/Triiodide Electrolyte ........ 78

Figure 41 Stirring of RNCaSil with Water Iodide/Triiodide Electrolyte in a Beaker Cell;

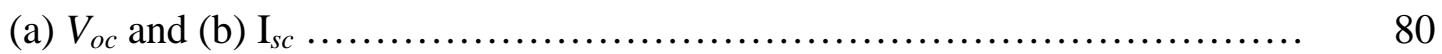

Figure 42 Varying RNCaSil Load in Water Iodide/Triiodide Electrolyte with 5 wt\%

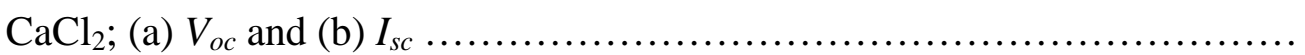

Figure 43 The Effect of $\mathrm{CaCl}_{2}$ content in the Iodide/Triiodide Electrolyte (100\%

Water); (a) $V_{o c}$ and (b) $I_{s c}$

Figure $44 \mathrm{Ca}^{2+}$ Solubility at Different $\mathrm{pHs}$

Figure 45 Iodide/Triiodide Electrolyte with Different Additives: 5 wt $\% \mathrm{CaCl}_{2}$ and, Relative to the $\mathrm{CaCl}_{2}$ Electrolyte, Equimolar Amounts of $\mathrm{SrCl}_{2}$ and $\mathrm{MgCl}_{2}$, and $55.7 \mathrm{~mol} \% \mathrm{Ca}^{2+}$; (a) $V_{o c}$ and (b) $I_{s c}$

Figure 46 RNCaSil with Tetrabutylammonium Hexafluorophosphate Compared to RNCaSil in Water Based Iodide/Triiodide Electrolyte $\left(5 \mathrm{wt} \% \mathrm{CaCl}_{2}\right) \ldots \ldots \ldots$

Figure 47 The Effect of Iodide/Triiodide Electrolyte Solvent - Plotted in Order of Increasing Viscosity of the Electrolyte Solution: (a) $V_{o c}$ and (b) $I_{s c} \ldots \ldots \ldots \ldots$ 
Figure 48 The Effect of Iodide/Triiodide Electrolyte Solvent - Plotted in Order of Increasing Dielectric Constant of the Dominant Solvent: (a) $V_{o c}$ and (b) $I_{s c} \ldots$

Figure 49 Beaker Tests of RNCaSil in Differently Solvated Iodide/Triiodide (30 wt\% water:70 wt $\%$ other); (a) $V_{o c}$, and (b) $I_{s c}$. Non-constant Illumination

Figure 50 The Effect of Adding $2 \mathrm{wt} \% \mathrm{CaCl}_{2}$ to the Electrolyte of Water or $70 \mathrm{wt} \%$ Ethanol (30\% Water) Iodide/Triidide Electrolytes, With and Without RNCaSil (Normal Gasket); (a) $V_{o c}$ and (b) $I_{s c}$

Figure 51 Effect of Solvent on RNCaSil Cells in $30 \mathrm{wt} \%$ Water Iodide/Triiodide, Including Dark Processes. Plotted in Order of Increasing Dielectric Constant of the Solvent; (a) $V_{o c}$ and (b) $I_{s c}$

Figure 52 The Effect of Cell Diameter on Cell Performance of RNCaSil in Water-Based Iodide/Triiodide ( $5 \mathrm{wt} \% \mathrm{CaCl}_{2}$ ) Electrolyte; (a) $V_{o c}$ and (b) $I_{s c} \ldots \ldots \ldots \ldots \ldots$

Figure 53 Effect of Electrode Distance with Different Loads of NCaSil to $1.0 \mathrm{~cm}^{3}$ Water-Based Iodide/Triiodide (5 wt $\% \mathrm{CaCl}_{2}$ ) Electrolyte; (a) $V_{o c}$ and (b) $I_{s c}$..

Figure 54 Effect of Electrode Distance with Different Loads of NCaSil to $1.0 \mathrm{~cm}^{3}$ Water-Based Iodide/Triiodide (5 wt $\% \mathrm{CaCl}_{2}$ ) Electrolyte, $I_{s c}\left(\mu \mathrm{A} / \mathrm{cm}^{3}\right)$

Figure 55 Effect of Electrode Distance on a RNCaSil and Water-Based Iodide/Triiodide $\left(5 \% \mathrm{CaCl}_{2}\right.$ ) Electrolyte Beaker Test (Ratio of $1.0 \mathrm{~g}: 10 \mathrm{~cm}^{3}$ ). Normal Set Up; the Electrodes were Perpendicular to the Light Beam with the C-coated ITO as the Dark Electrode

Figure 56 Effect of Electrode Distance on a RNCaSil and Water-Based Iodide/Triiodide $\left(5 \% \mathrm{CaCl}_{2}\right.$ ) Electrolyte Beaker Test (Ratio of $1.0 \mathrm{~g}: 10 \mathrm{~cm}^{3}$ ): (a) Electrodes Parallel to the Light Beam; and (b) Electrodes Perpendicular to the Light Beam with the C-coated ITO as the Photoelectrode

Figure 57 Slotted Vessel - The Gap Between the Quartz Glass and Photoelectrode can be Unfilled or Filled with Sample

Figure 58 Effect of Distance on (a) $V_{o c}$ and (b) $I_{s c}$ on RNCaSil in $70 \mathrm{wt} \% 30 \mathrm{wt} \%$

Water-Based Iodide/Triiodide Electrolyte

Figure 59 Speculated Reason Why the Open-Circuit Potential Appears to Increase Upon Back Reflection

Figure 60 Effect of Cell Backing with RNCaSil in a Water Iodide/Triiodide Electrolyte (5 wt $\% \mathrm{CaCl}_{2}$ ); (a) $V_{o c}$ and (b) $I_{s c}$ in $\mu \mathrm{A} / \mathrm{cm}^{2}$

Figure 61 Effect of Cell Backing with RNCaSil in a Water Iodide/Triiodide Electrolyte (5 wt $\% \mathrm{CaCl}_{2}$ ); $I_{s c}$ in $\mu \mathrm{A} / \mathrm{cm}^{3}$.

Figure 62

The Increase in Current Could Increase Corresponding Voltages ....

Figure 63 RNCaSil with Water-Based Iodide/Triiodide in Gasket Tests with Different 
Back-Electrode Coatings; (a) $V_{o c}$ and (b) $I_{s c}$

Figure 64 Current versus Voltage of RNCaSil in $70 \%$ Ethanol $30 \%$ Water

Iodide/Triiodide: C-Tape Coated Normal Glass Compared to C-coated ITO

Glass, Back Electrode

Figure 65 RNCaSil in Water Iodide/Triiodide Electrolyte (5 wt\% $\mathrm{CaCl}_{2}$ ). Beaker cells

with Electrode Distances of: (a) $0.5 \mathrm{~cm}$; (b) $2.0 \mathrm{~cm}$; and (c) $2.5 \mathrm{~cm}$

Figure 66 Current versus Voltage of $\mathrm{I}_{2}-\mathrm{RNCaSil}$ Beaker Sample, Compared to the

Equivalent Normal $\mathrm{I}_{2}-\mathrm{RNCaSil}$ and RNCaSil Cells. All After 24 Hours

Stirring in the Dark

Figure 67 RNCaSil with $70 \%$ Ethanol $30 \%$ Water Iodide/Triiodide Electrolyte in a

Quartz Cuvette, with Different Electrode Combinations; (a) $V_{o c}$ and (b) $I_{s c} \ldots$.

Figure 68

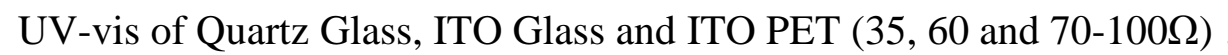

Figure $69 \mathrm{RNCaSil}$ in Water Iodide/Triiodide Electrolyte $\left(5 \mathrm{wt} \% \mathrm{CaCl}_{2}\right)$ in Different

Electrodes; (a) $I_{s c}$ and (b) $V_{o c}$

Figure 70 Side-On Representation of (a) ITO PET; and (b) ITO-Glass Cells

Figure 71

Current over Voltage Plots of g-NCaSil in ITO-PET cells; with Tin or Copper

Contacts, after 5 or 10 Days of Gel Formation, Laminated or Not

Figure 72 UV-vis of the Laminated Plastic Used to Laminate the Cell Compared to the ITO PET Used

Figure 73 Long-Term Testing of RNCaSil in $30 \mathrm{wt} \%$ Water $70 \mathrm{wt} \%$ Other Solvent

Iodide/Triiodide Electrolyte; (a) $V_{o c}$ and (b) $I_{s c}$

Figure 7472 Hours Illumination of RNCaSil in 70 wt\% Ethoxy Ethanol, Ethylene

Glycol and Ethanol Iodide/Triiodide Electrolytes

Figure 75 Constant Illumination of the $70 \mathrm{wt} \%$ Ethanol Iodide/Triiodide Electrolyte; (a) $V_{o c}$ and (b) $I_{s c}$

Figure 76 Constant Illumination of RNCaSil (Batch \#4) in $70 \mathrm{wt} \%$ Ethanol

Iodide/Triiodide Electrolyte, After 24 or 72 Hours Settling Time; (a) $V_{o c}$ and (b) $I_{s c}$

Figure 77 Constant Illumination of $\mathrm{I}_{2}$-EENCaSil in $70 \mathrm{wt} \%$ Ethanol Iodide/Triiodide.

After 24 and 72 Hours Settling; (a) $V_{o c}$ and (b) $I_{s c}$

Figure $78 \mathrm{~g}-\mathrm{NCaSil}$ 14, 21 and 28 Days After Formation, Compared to a $70 \mathrm{wt} \%$

Ethanol $30 \mathrm{wt} \%$ Water Iodide/Triiodide Electrolyte and an Equivalent

RNCaSil-Based Cell; (a) $I_{s c}$ and (b) $V_{o c}$

Figure 79 g-NCaSil (dried at $55^{\circ} \mathrm{C}$ ) and RNCaSil with Water-Based Iodide/Triiodide $(5$

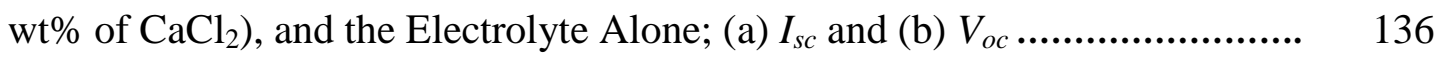

Figure 80 Cells from Gel-State V-NCaSil and Ce-NCaSil; (a) $V_{o c}$ and (b) $I_{s c} \ldots \ldots \ldots \ldots$ 
Figure 81

Figure 82

Figure 83

Figure 84

Figure 85

Figure 86

Figure 87

Figure 88

Figure 89

Figure 90

Figure 91

Figure 92

Figure 93

Figure 94

Figure 95

Figure 96

Figure 97

Figure 98

Figure 99

Figure 100

Figure 101

Figure 102

Figure 103

Figure 104

Figure 105

Figure 106

Figure 107
g-NCaSil after 144 hours Illumination; (a) $V_{o c}$ and (b) $I_{s c}$

139

${ }^{29} \mathrm{Si}$ Solid State NMR Spectra of g-NCaSil, RNCaSil and Untreated NCaSil ... 140 (a) $V_{o c}$ and (b) $I_{s c}$ of Cation Treated TEOS gels (Pt-Coated Back Electrode) ... 142 Silicate Derived Gels Infused with Different Metal Sources (Pt-

Coated Back Electrode); (a) $V_{o c}$ and (b) $I_{s c}$

(a) Structure of NCaSil; and (b) Proposed Structure of g-NCaSil

After 168 Hours Illumination

Ethanol (30 wt\%Water) Iodide/Triiodide

wt $\%$ Water Iodide/Triiodide

Iodide/Triiodide and (b) $I_{s c}$ 161

Particle Size Distribution Analysis of $\mathrm{TiO}_{2}$ and $\mathrm{SiO}_{2}$

IR Spectrum of RNCaSil Alone and With Water-Based Iodide/Triiodide (0 and $5 \mathrm{wt} \% \mathrm{CaCl}_{2}$ ) 


\section{LIST OF TABLES}

Table 1 Different Methods for Theoretical Porosity and $I_{s c} / V_{o c}$ Calculations ................. 11

Table 2 Hara et al.'s Results for Iodide Compared to Bromide in Acetonitrile with $\mathrm{TiO}_{2}$ DSSCs

Table 3 Effect of Cations on Cell Performance in Acetonitrile (Hara et al.) with $\mathrm{TiO}_{2}$ DSSCs

Table 4 Optical Properties of Different Types of NCaSil (data from PhD thesis of Dr Andy McFarlane, School of Chemical and Physical Sciences, Victoria University of Wellington)

Table 5 Formulation of Iodide/Triiodide Electrolytes from Different Solvents 41

Table 6 Formulation of the Normal Iodide/Triiodide Electrolyte Used 45

Table 7 Optical Density Filters and their Corresponding Light Intensities 55

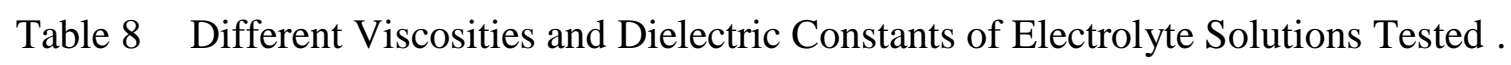
90

Table 9 Solubility of $\mathrm{Ca}^{2+}$ Dependent on the Concentration of $\mathrm{OH}^{-}$ 173 


\section{GLOSSARY}

Dark Current

Donor Number

Fill factor

Incident photon-to-current conversion efficiency

(IPCE)

Junction

Light path

Maximum power (mp)

Open-circuit voltage, $V_{o c}$

Photogalvanic

Photovoltaic
The dark current is a form of cell inefficiency and is the current through a device at a given bias, in the absence of light. It opposes the photocurrent.

This expresses a molecules ability to share the electron density of its lone electron pairs, i.e., it is a measure of lewis basicity.

The fill factor is a measure of cell performance, and is the $(I \times V)$ at maximum power, over the $\left(I_{s c} \times V_{o c}\right)$.

The quantum efficiency of photocurrent generation from incident photons, i.e., the number of electrons extracted per incident photon.

A junction is the interface shared by which two different materials.

The light path is the length, which a beam of light can travel within a cell.

Power is defined as $(I \times V)$, thus the maximum power is the maximum value of $(I \times V)$ the cell is capable of.

The open-circuit voltgate is the difference of electrical potential between two terminals of a device when there is no external load connected $(I=0)$.

Photogalvanic describes a process which converts light into energy via an electrochemical process. Charges are photochemically generated in a solution-phase redox couple.

Photovoltaic describes a process which converts light to electricity by the creation of an electron/hole pair. 
Power conversion

efficiency

Short-circuit current, $I_{s c}$

Trap State
The power conversion efficiency is a measure of overall cell efficiency, which compares the amount of power obtained from the cell relative to the amount of power put in.

The short-circuit current is measured when resistance and potential approach zero.

A trap state refers to a state of higher or lower energy, from in which an electron might never produce an output, unless there is a driving force. 


\section{INTRODUCTION}

The increasing demand for energy over the past fifty years has resulted in a drive for research into possible energy sources that are both commercially viable and do not create waste products that are detrimental to the environment. ${ }^{[1]}$ It has been projected that, by 2050 , worldwide energy consumption will increase to approximately $28 \mathrm{TW}$, from its 2006 level of around $11 \mathrm{TW}{ }^{[2]}$ With a potential of approximately $100000 \mathrm{TW}$, the solar energy that reaches the Earth's surface is the largest carbon-free energy source ${ }^{[2]}$ which could be harvested with photovoltaic or photogalvanic devices.

Current silicon based solar cells on the market, based on inorganic solid-state junction devices, are environmentally clean, but expensive ${ }^{[3,4]}$ due to large amounts of materials required for production, and generally heavy or cumbersome. ${ }^{[5]}$ Though silicon cells have relatively high reported power conversion efficiencies of between 15 and $20 \%,{ }^{[6]}$ the efficiencies achieved in laboratory studies are often not conveyed in commercially available applications, due to problems with scale up and the requirement of highly controlled conditions. ${ }^{[7]}$

Silicon-based cells have dominated the photovoltaic market for the last fifty years and much research is still made into enhancing existing systems. ${ }^{[7]}$ However, silicon cells have a theoretical maximum power conversion capacity of less than $29 \% .^{[8]}$ Alternate forms of cells are thus being heavily researched, particularly those using nanomaterials, due to the favourably small amount of material needed and the likely associated decrease in cost per watt.

\section{Dye-Sensitised Solar Cells (DSSCs)}

Dye-sensitised solar cells (DSSCs) are an alternative to silicon devices and have solid-liquid junctions. So far, relatively high levels of power conversion above $10 \%$ have been achieved with dye-sensitised $\mathrm{TiO}_{2}$ nanoparticle cells, ${ }^{[9]}$ but no DSSC has managed to achieve conversions able to compete with silicon based cells. For example, the most well known DSSC, Graetzel's Cell (a $\mathrm{TiO}_{2}$, ruthenium dyesensitised, I/I $\mathrm{I}^{-}$electrolyte cell), has been made with a power conversion efficiency of $12 \% .{ }^{[10]}$ DSSCs are additionally advantageous in their insensitivity to temperature changes. It has been shown that increasing the temperature from 20 to $60{ }^{\circ} \mathrm{C}$ has little effect on the power conversion of a DSSC, whereas silicon cells show a decrease in power conversion of about $20 \%$ under the same conditions. ${ }^{[11]}$ 
DSSCs comprise of three principle components: a light-absorbing material, an electron-conducting material and a hole-conducting material. In a non-dye-sensitised cell, the light absorbing and electronconducting materials are the same. In a dye-sensitised cell, it is the dye that absorbs the light, creating an electron/hole pair. ${ }^{[12]}$ Thus, the efficiency of solar cells depends on:
A. the spectral absorbance of the light-absorbing material and the electron/hole injection efficiency;
B. the electron transport and recombination rates, which are dependent on the electron-conducting material; and
C. the hole transport and stability, which is related to the hole conductor used.

\subsection{Electrical Contacts}

\subsubsection{Cell Make-up}

The three major components (semiconducting material, dye sensitiser and hole-transporting material) of a DSSC are sandwiched between two electrical contacts or electrodes. On illumination and creation of electron/hole pairs, electrons injected into an electron-conducting material flow through to the anode and return through the cathode creating an external current, which can be used by a load. Once at the cathode, the electron reduces the oxidised hole conductor, restoring it to its original state (Figure 1). The two electrical contacts are predominantly made of glass coated with either indium tin oxide (ITO) or fluorine-doped tin oxide $\left(\mathrm{F}_{\mathrm{x}} \bullet \mathrm{SnO}_{2}\right)$. Thin films of etched $\mathrm{ZnO}: \mathrm{Al}$ on glass have also been used for silicon-based solar cells. ${ }^{[13,14]}$ Recently, DSSC work has been carried out on plastic electrodes based on poly(ethylene terephthalate). ${ }^{[15]}$

\subsubsection{The Photoelectrode (Anode)}

The anode is the electron-conducting electrode, also named the photoelectrode as it is the illuminated electrode. Electron-conducting materials are usually placed onto the anode in such a way as to maximise contact. This is usually achieved by doctor blading, ${ }^{[6,16-19]}$ screen printing, ${ }^{[6,10,12,17,20]}$, spin coating, ${ }^{[21]}$ or sol-gel deposition of dissolved electron-conducting material together with a binder. ${ }^{[6,22]}$ The contact and electron-conducting material are fired (in air at 450 to $500{ }^{\circ} \mathrm{C}$ for $\mathrm{TiO}_{2}$ ) to sinter the electron-conducting particles to each other and to the substrate. ${ }^{[12,23,24]}$ Sintering removes the binder 
and solvent to create an electronically connected electron-conducting network. ${ }^{[15]}$ This is then usually immersed in a solution of the light sensitiser to coat the electron-conducting material with the sensitiser. ${ }^{[10,18,25]}$

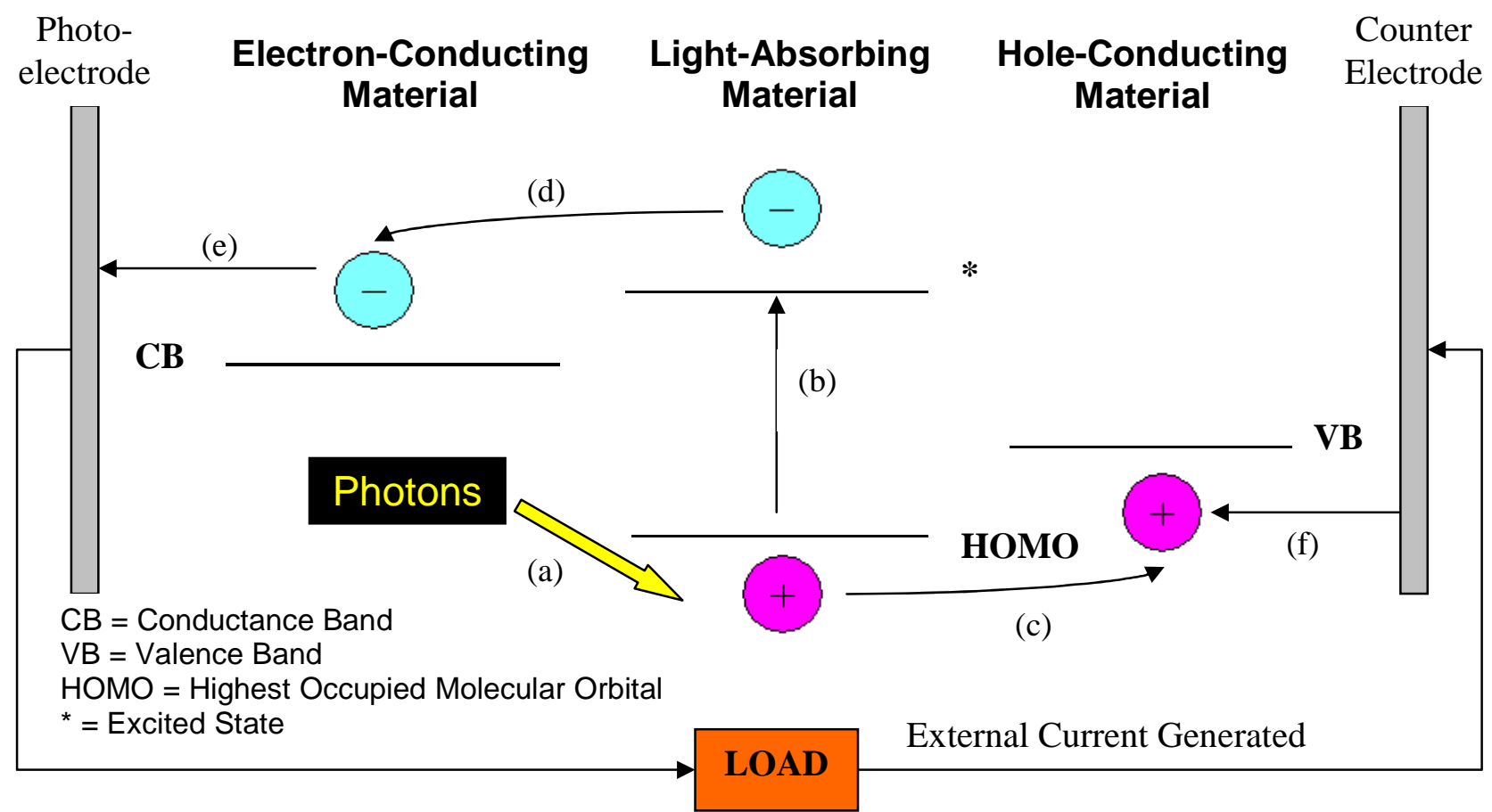

Figure 1. Generalised Electron and Hole Orbital and Cell Movement in DSSCs: (a) Photoexitation of the LightAbsorbing Material; (b) Electron/Hole Pair Photogeneration; (c) Movement of the Generated Hole from the HOMO of the Light-Absorbing Material to the VB of the Hole-Conducting Material via the Transfer of an Electron from the

Latter to the Former; (d) Electron Flow from the Excited State of the Light-Absorbing Material to the CB of the Electron Conducting Material; (e) Electron Flow Through the Photoelectrode and the External Circuit, Re-entering Through the Counter Electrode; (f) Regeneration of the Hole-Conducting Material.

\subsubsection{The Counter Electrode (Cathode)}

Both electrodes are exposed to the hole conductor in the cell. ${ }^{[26]}$ Interaction between the hole conductor and the anode is undesirable, as it results in recombination. Thus, interaction at the opposite electrode (the cathode) must be faster, to make it kinetically more favourable. Because of this, the cathode (for example, ITO glass) is usually coated with either carbon, ${ }^{[9]}$ platinum $^{[16,24,25,27]}$ or gold. ${ }^{[10,18,21]}$ Poly(3,4-ethylenedioxythiophene) (PEDOT) coated $\mathrm{F}_{\mathrm{x}} \bullet \mathrm{SnO}_{2}$ glass is also an efficient counter electrode used in $\mathrm{TiO}_{2}$ DSSCs. ${ }^{[26]}$ The coating on the cathode catalyses the formation of iodide from triiodide, regenerating the electrolyte. ${ }^{[9]}$ It has been found that coating the back electrode of DSSCs with platinum greatly improves cell performance. ${ }^{[28]}$ The cathode is adjusted to accommodate an equilibrium 
potential close to that of the hole conductor's, whereas the anode (the photoelectrode) is far from the equilibrium potential. ${ }^{[26]}$

It is possible that such a coating would not be required for cells that do not have iodide/triiodide based hole conductors. However, to date, reports of cells where polymer hole conductors have been used still required a coating of the cathode. ${ }^{[10,18,19,22]}$

\subsubsection{Plastic Electrodes}

A major drawback of using glass electrodes is that they lack flexibility and are relatively heavy. Because of this, lighter, impact resistant and more flexible plastic electrodes based on ITO-coated poly(ethylene terephthalate) (ITO PET) have been investigated. ${ }^{[15,29]}$ The use of flexible electrodes would also allow DSSCs to be made using a continuous roll-to-roll, high speed coating process and cells could be made in different shapes for specific surfaces. ${ }^{[15]}$ The ability to manufacture DSSCs in continuous steps, as opposed to the batch process required with glass, should lower production costs. ${ }^{[15]}$ That such composed cells could simply be laminated together has also been considered. ${ }^{[29]}$

So far, ITO PET DSSCs have not been able to compete with equivalent glass cells, but completely solid-state cells made with ITO PET have been reported to have achieve power conversion efficiencies of $5.5 \% .{ }^{[30]}$ A major challenge with plastic electrodes is that they cannot be sintered above $150{ }^{\circ} \mathrm{C}$ without polymer degradation, causing loss of transparency and shape. ${ }^{[15]}$ Consequently, there is poor electrical adhesion between the electron-conducting particles, low dye adsorption, and residues of the binder and solvent remain. ${ }^{[15]}$ Furthermore, though the PET may be flexible, the nanocrystalline semiconducting materials often are not, and have been known to "crack" or peel away from the conductive substrate. ${ }^{[31]}$ A possible solution to this is the use of nanowires grown on the substrate. ${ }^{[31]}$

\subsection{Light-Absorbing Materials}

To increase the range of spectral absorbance, dye sensitisers have been used and treated onto the surface of electron-conducting materials as a monolayer ${ }^{[11]}$ (multilayers of dye have been found to be unhelpful in increasing light harvesting). ${ }^{[32]}$ In DSSCs, it is the sensitising material that absorbs the light (photons) and photo-induced charge separation occurs at the interface between the electron conductor and "hole" carriers. ${ }^{[17]}$ This charge separation occurs because, on absorption of a photon, 
electrons are excited from the HOMO to an excited state in the dye, creating an electron/hole pair. The electrons move into the electron conductor, and the hole into the hole conductor. For separation at the sensitiser, the energy of the photon must be greater than the energy of the band gap in the material.

Unlike with silicon cells, which create charge separation through electric fields present in $p$ - and $n$ doped electron-conducting material, DSSCs achieve charge separation via kinetic competition (Part I 1.2.1), as in photosynthesis. ${ }^{[2]}$

The light-absorbing materials in solar cells must efficiently generate electrons and holes in the structure (see above, Figure 1(a) and (b)). ${ }^{[33]}$ Due to the second law of thermodynamics, not more than $33 \%$ efficiency can be achieved by a solar converter with only one pigment. ${ }^{[9,34,35]}$ When only a single pigments has been used, Ru-based dyes have achieved the best results. The ideal sensitiser would be able to absorb light at all wavelengths $(\lambda)$ below $920 \mathrm{~nm},{ }^{[1]}$ thus spanning across the range of light that reaches the Earth's surface, and maximising efficiency. Absorption in the near-IR or red region are preferred, ${ }^{[3]}$ as up to $80 \%$ of incident light that reaches Earth is red shifted. ${ }^{[36]}$ The concept of dyesensitising $\mathrm{TiO}_{2}$ had been considered for many years, ${ }^{[37]}$ but prior to Graetzel's cells ${ }^{[32]}$ no dye had proven to satisfy all the requirements of a sensitiser.

The absorbance range can be modified by the use of different sensitisers. ${ }^{[25]}$ In selecting a sensitiser, the energy level of the excited state must be higher than the lowest edge of the conduction band of the electron conductor. Additionally, the ground state must be below the upper edge of the valence band of the hole conductor. This is necessary so that the electron can be passed from the dye to the electron conductor, and the hole from the dye to the hole conductor.

The ideal sensitiser should be stable and be able to attach to the surface of the electron-conducting material (for example, attachment via carboxylate or phosphonate groups on the sensitiser to the $\mathrm{Ti}^{4+}$ in $\left.\mathrm{TiO}_{2}\right) .{ }^{[3]}$ Generally, carboxylate linkers have achieved greater efficiencies than phosphonate linkers. ${ }^{[3]}$ The carboxylate groups are able to form ester linkages with the oxides on the electron conductor's surface, creating a strong bond between the sensitiser and electron conductor. ${ }^{[3]}$ However, as the bond can be hydrolysed by water, the cells require non-aqueous conditions. ${ }^{[3]}$ Recent studies have shown that hydrophobic dyes are able to retard hydrolysis, preventing dye desorption. ${ }^{[38-40]}$ Furthermore, it is postulated that the hydrophobic sensitiser can prevent the hole conductor interacting with the surface of the electron-conducting material, thus hindering recombination of generated electron/hole pairs. ${ }^{[3]}$ 
Sensitisers should also have redox potentials sufficiently high, so that they can be regenerated by electron donation from the hole conductor. ${ }^{[17]}$

\subsubsection{Organometallic Dyes}

Sensitiser requirements are met by some organometallic dyes - usually ruthenium or osmium complexes. ${ }^{[1]}$ Both osmium and ruthenium are known to yield luminescent complexes that have have very favourable electrochemical, photophysical and photochemical properties. ${ }^{[41,42]}$ A variety of different complexes have been tested, but not all have had great success. Graetzel et al. ${ }^{[1,17]}$ have discovered three ruthenium based dyes that have proven to work well in the DSSC. These are the "N3" or "N719" dyes, (cis-bis(isothiocyanato)bis(4,4'-dicarboxylato-2,2'-bipyridyl)-Ru(II)) $)^{2-}$, where N3 is the acid form, the N719 has two tetra- $n$-butlyammonium (TBA) groups on the carboxylate groups; and "Black Dye" (tri(cyanato)-4,4',4”-tricarboxylato-2,2',2"'-terpyridyl- Ru(II)•2TBA) (Figure 2). These dyes have achieved power conversion efficiencies of over $10 \%$ and are able to sustain $10^{8}$ redox cycles and 20 years of continuous use in normal sunlight. ${ }^{[11]}$

Graetzel et al. ${ }^{[17]}$ achieved photocurrents of 16.5 and $20.5 \mathrm{~mA} / \mathrm{cm}^{2}$ with the $\mathrm{N} 3$ and Black Dye respectively. That the dyes achieve such good results is not surprising, given that their wide spectral absorbance ranges span the visible spectrum (Figure 3). This greatly enhances the spectral range of the cell, as the $\mathrm{TiO}_{2}$ absorbance falls off sharply at $400 \mathrm{~nm}$. Furthermore, it is not surprising that the Black Dye performs better than N3 dye, seeing that it absorbs all the way up to $>900 \mathrm{~nm}$, whereas N3 stops absorbing at below $800 \mathrm{~nm}$.
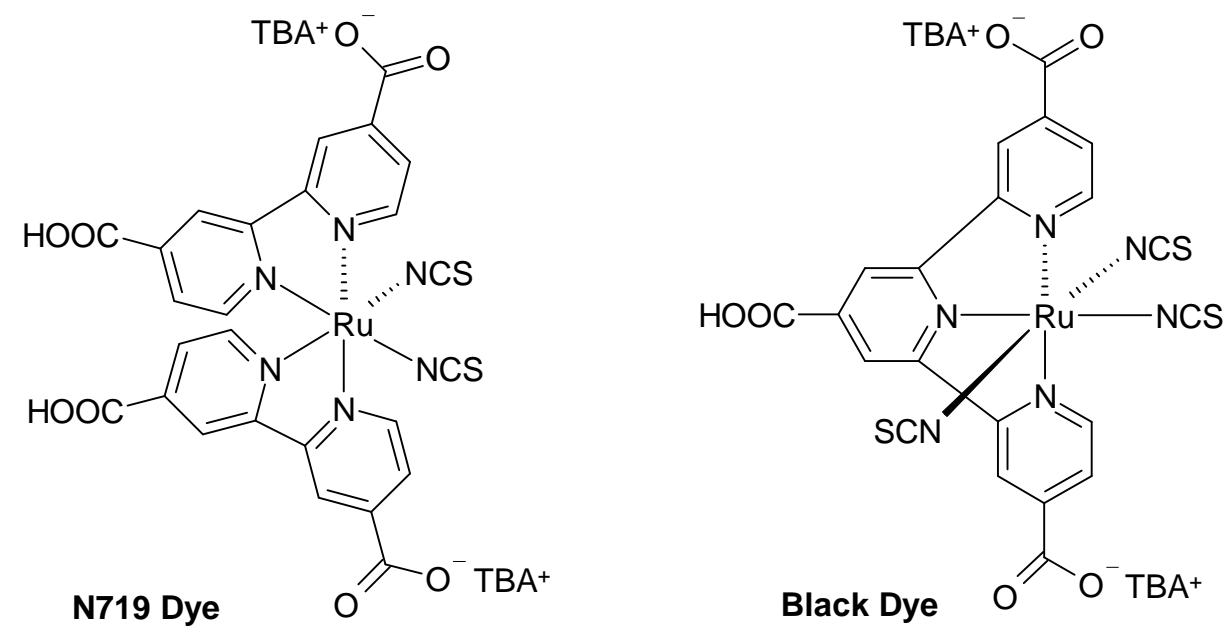

Figure 2. N719 and Black Dye used by Graetzel et al. 


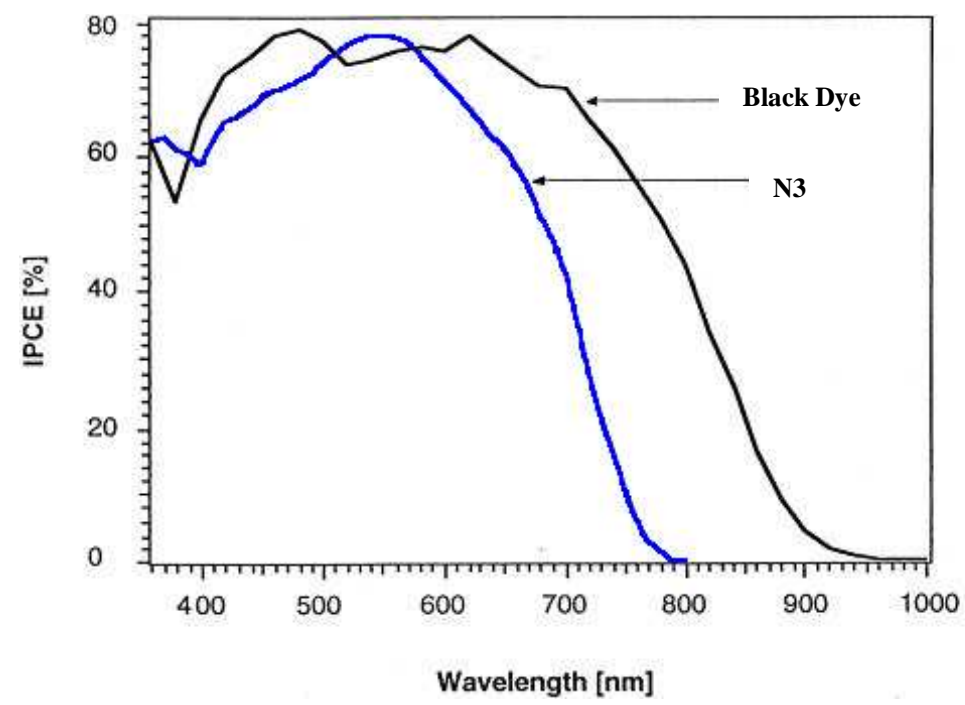

Figure 3. Spectral Absorbance Ranges of the N3 and Black Dye, as Inferred from the IPCE (IPCE is the Incident Photon-to-Current Conversion Efficiency).

These ruthenium dyes have good charge separation, ideal for photovoltaic use. Absorption of low energy visible region light by the dyes results in a metal-to-ligand charge transfer transition state, in which the excited electron is located at the imine group. ${ }^{[3]}$ Because it is the ligands that attach to the $\mathrm{TiO}_{2}$, the result is fast injection of the electron into the electron-conducting material. The hole (or positive charge density) remains on the dye, distributed over the metal and to some extent over the NCS ligands (Figure 2). ${ }^{[3]}$ Thus, there is spatial separation of the hole and electron pair, thereby retarding recombination. The driving force of the process is kinetic rather than thermodynamic, the latter of which favours recombination as determined by the reduction potential of the hole/dye. Once the electron is injected into the electron conductor, the oxidised sensitiser is stable within the time required for diffusion of the hole conductor and reduction by it. ${ }^{[43]}$ The oxidised sensitiser undergoes substitution of its NCS ligands with solvent molecules with a rate constant in the order of $10^{4} \mathrm{~s}^{-1}$, whereas hole conductance occurs within $10 \mathrm{~ns}^{[43]}$

Studies have also been carried out on the positioning of the linker groups on the ruthenium dyes. In N3 the linker groups are on the 4,4' positions of the bipyridyl ligand. 3,3' positioning has been assessed, and it has been shown that this results in a decrease in efficiency. ${ }^{[44]}$ This has been attributed to the 3,3' positioning requiring the complex to twist, changing its electrochemical properties. ${ }^{[44]}$ Systems based on N3/N719, but with variations of the carboxylate groups on the 4,4' position on one of the bipyridyl groups have proven to work quite well, ${ }^{[3,45,46]}$ for example: cis-bis(isothiocyanato)(4,4'-dicarboxylic acid-2,2'-bipyridine)(4,4'-bis( $p$-hexyloxystyryl)-2,2'-bipyridine) Ru(II), or “K-19”. ${ }^{45]}$ 
The $\pi$-conjugated systems in these dyes are important for charge separation, and expansion of the $\mathrm{C}=\mathrm{C}$ backbone can extend the absorption spectrum into the red region, thus improving cell performance. ${ }^{\text {[47] }}$ Doing so with $\pi$-conjugated rings both increases cell performance and cell stability. ${ }^{[23]}$ The type of ring used (for example, thiophene or phenyl) ${ }^{[23]}$ affects the redox potential of the dyes greatly, also affecting cell performance. ${ }^{[23]}$ If one of the 2,2'-bipyridyl-4,4-dicarboxylate groups on the N3 dye is exchanged for a styryl-substituted bipyridine, the extinction coefficient of the dye is increased and additionally the dye becomes more hydrophobic and, thus, less likely to be desorbed from the electron conductor. ${ }^{[40]}$

\subsection{Electron-Conducting Material}

In DSSCs, the electrons generated are transferred into an electron-conducting material (above, Figure 1(d)), where the electrons are free to flow through the conduction band to an electrode. Electrons are converted to electricity in a process termed the photovoltaic effect. The electron conductor must have a conduction band energy that allows for efficient electronic coupling with the sensitiser energy levels and charge separation, and to minimise recombination of the electron and sensitiser. An electronconductor system that has a low level of recombination and maintains a good electrical connectivity to the electrode/substrate is the most favourable. ${ }^{[25]}$

$\mathrm{TiO}_{2}$ (anatase) satisfies these restrictions and much research has been done with mesoporous $\mathrm{TiO}_{2}$ nanoparticles in photovoltaics, with the most successful being a $10 \mu \mathrm{m}$ thick porous film made up of particles of $15 \mathrm{~nm}$ in diameter. ${ }^{[25]}$ The success of the $\mathrm{TiO}_{2}$ DSSCs has been attributed to the fast electron injection from the sensitiser to the $\mathrm{TiO}_{2}(\sim 50 \mathrm{fs})$ and a much slower reversal process. ${ }^{[48]}$ This high speed is consistent with near $100 \%$ quantum efficiency. ${ }^{[28]}$ As the lifetime of the excited state of most dyes (for example, N3) is around $50 \mathrm{ns,} \mathrm{efficient} \mathrm{injection} \mathrm{could} \mathrm{still} \mathrm{take} \mathrm{place} \mathrm{if} \mathrm{the} \mathrm{injection}$ were magnitudes slower. There is no necessity that the injection be so much greater than the rate of recombination; this is known as "kinetic redundancy". ${ }^{[3]}$ Such an effect is actually inefficient and efficiency is maximised when the rate of injection is only just faster than the rate of recombination. ${ }^{[3]}$ The kinetic redundancy can be modified by changing the sensitiser-electron-conductor linker groups. ${ }^{[3]}$

Anatase is favoured over rutile because it is the low-temperature stable form (kinetically favoured) and forms transparent and colourless mesoscopic films. ${ }^{[12]}$ It has better light scattering properties than rutile and it has been found that the use of anatase over rutile improves efficiency by a factor of five. ${ }^{\text {[28] }}$ Furthermore, rutile has a surface area $25 \%$ lower than anatase and absorbs around $35 \%$ less dye. ${ }^{[28]}$ 
$\mathrm{TiO}_{2}$ is a wide band gap material, with a band gap of $3.2 \mathrm{eV} \cdot{ }^{[5]}$ Alternative wide band gap materials have also been studied, such as $\mathrm{ZnO}^{[16,25,27,49,50]}, \mathrm{Nb}_{2} \mathrm{O}_{5},{ }^{[16,51]}, \mathrm{SnO}_{2}{ }^{[16]}$ and $\mathrm{In}_{2} \mathrm{O}_{3} .{ }^{[16]}$ Wide band gap materials tend to be insensitive to the visible spectrum and, generally, only absorb high energy UV wavelengths. However, a wide band gap is desirable, as it implies strong chemical bonds and thus a stable material. ${ }^{[5]}$ It also implies that the material is transparent to visible light, allowing maximum uptake by the sensitiser in this region, which is desirable as sensitisers tend to be more efficient at creating stable electron/hole pairs than standard electron-conducting materials. If the electronconducting material itself had a wide spectral range, a sensitiser would not be needed.

Solar cells made of polycrystalline films (10 to $20 \mu \mathrm{m}$ thick $)^{[28]}$ of randomly connected oxide nanoparticles (10 to $30 \mathrm{~nm}$ in diameter ${ }^{[28]}$, such as in nanocrystalline $\mathrm{TiO}_{2}$, are more efficient than non-nanoparticle compacted layers, because the internal surface area is about one hundred times larger, as determined by their "roughness". ${ }^{[10]}$ A high roughness of particles means that these particles scatter light to a high degree. ${ }^{[10]}$ This scattering increases the optical path length of the incident light beam that passes through the sample, which results in a high optical density of the dye-coated nanoparticle layers. A greater surface area is favourable in dye-sensitised cells, as it also increases the area of the dye, which is absorbed onto the surface of the electron-conducting material. ${ }^{[3]}$ The film must be mesoporous so that the hole conductor can diffuse through it. ${ }^{[52]}$

\subsubsection{Cell Thickness}

Ito et $a l .{ }^{[53]}$ have found that, in a liquid-electrolyte $\mathrm{N} 719$-dyed $\mathrm{TiO}_{2}$ cell, the greater the cell thickness (and thus surface area), the more sites there are for charge recombination. The more particles there are the more difficult it is to coat the sample completely and evenly with the sensitiser. They found a disproportionately larger number of uncoated sites in materials with larger surface areas. Hence, they noted an increase in dark current causing the open-circuit voltage ( $V_{o c}$, see Glossary) to drop.

Ito et al. $^{[53]}$ also observed a rise in short-circuit current ( $I_{s c}$, see Glossary) with increasing cell thickness, with the current reaching a maximum at a certain thickness $(14.0 \mu \mathrm{m})$ before falling off. Beyond the maximum point, the $I_{s c}$ deceases as the electrons and holes have to travel greater distances. ${ }^{[10]}$ Overall power conversion efficiencies were found to improve on increasing cell thickness up to a thickness of $12.6 \mu \mathrm{m}$ while maintaining the cell's fill factor (ff, a measure of cell efficiency, see Part I 5.1). 
These findings were confirmed by Hara et al. ${ }^{[16]}$ who used a mercurochrome sensitiser. Hara et al. showed that as the cell thickness increases $V_{o c}$ drops and $I_{s c}$ increases. The rise in current was said to be due to an increase in the amount of absorbed dye for thicker cells. Conversely, the voltage decreases as the cell thickness increases. This was attributed to a loss of injected electrons due to an accretion in recombination and growing series resistance of the cell. ${ }^{[16]}$ The increase in recombination was argued to be due to there being more non-dye absorbed $\mathrm{TiO}_{2}$ sites able to interact with the hole conducting material. ${ }^{[16]}$

Cell thickness is also important in how it affects light scattering, optical path length and optical density. ${ }^{[10]}$ At $5 \mu \mathrm{m}$ thickness, it has been found that $\mathrm{TiO}_{2}$ reaches near $100 \%$ incident photon-tocurrent conversion efficiency (IPCE) in the blue spectral range. ${ }^{[10]}$

\subsubsection{Porosity Effects}

The internal surface area of an electron-conducting material is dependant on particle size and film thickness. ${ }^{[32]}$ Smaller particles will have greater accessible porosity. The average coordination number of a particle decreases when its porosity increases. For $\mathrm{TiO}_{2}$, coordination decreases from 6.6 to 2.8 when porosity advances from $40 \%$ to $80 \% .{ }^{[28]}$ The greater the porosity, the greater the dye-electronconductor contact area will be due to a larger surface area. However, this must be balanced against the ability of the hole conductor to diffuse through the pores. ${ }^{[32]}$

Porosity affects DSSC performance by changing both the light absorption coefficient $(\alpha)$ and electron diffusion coefficient (D). ${ }^{[54]}$ As $\alpha$ increases, both $I_{s c}$ and $V_{o c}$ increase due to the absorption of more photons. As D increases, $I_{s c}$ is enhanced and $V_{o c}$ decreases. This is because, as $\mathrm{D}$ increases, the diffusion path is extended, which causes more electrons to be extracted into the external circuit, increasing the current. However, electron density is lowered, so the cell voltage decreases. ${ }^{[54]}$ The diffusion coefficient can be changed by porosity to maximise cell power $(\mathrm{I} \times \mathrm{V}){ }^{[55]}$

When current and voltage are modelled by the "constant overlap" ${ }^{[54]}$ method (Table 1) for a porosity range of 0.41 to 0.70 , it is assumed that all the $\mathrm{TiO}_{2}$ nanoparticles are of the same spherical shape and have a $10 \mathrm{~nm}$ radius. As the porosity is lowered, $I_{s c}$ increases and $V_{o c}$ changes very little. Within this porosity range, there are more $\mathrm{TiO}_{2}$ nanoparticles in the porous thin film, resulting in a higher $\alpha$ and $\mathrm{D}$. The overall power output is enhanced as the porosity decreases. Results based on this theoretical model fit well with experimental results. ${ }^{[56,57]}$ 
For porosities below 0.41 , modelling is done using the "variable overlap" method. ${ }^{[54]}$ As porosity decreases there is an increase in particle overlap, which causes a reduction in internal surface area, resulting in a small $\alpha$. An increase in the overlap allows for better electron diffusion leading to a rise in D. The overall effect of a smaller $\alpha$ and a larger $\mathrm{D}$ is that both $I_{s c}$ and $V_{o c}$ decrease. As the overlap increases and pore size decreases, the ability of the hole conductor to mediate between the particles is limited. This in turn heightens the likelihood of recombination. Thus, the actual $I_{s c}$ and $V_{o c}$ may be lower than predicted by the variable overlap model. ${ }^{[54]}$

Table 1. Different Methods for Theoretical Porosity and $I_{s c} / V_{o c}$ Calculations.

\begin{tabular}{lcc}
\hline Method & $\begin{array}{c}\text { Absorption Coefficient } \\
(\boldsymbol{\alpha})\end{array}$ & Electron Diffusion Coefficient $(\mathrm{D})$ \\
\hline $\begin{array}{l}\text { Constant Overlap } \\
\text { Variable Overlap }\end{array}$ & $\alpha=2568(1-\mathrm{P})(\mathrm{P}+2.89)$ & $\mathrm{D}=a\left|\mathrm{P}-\mathrm{P}_{\mathrm{c}}\right|^{\mu}$ \\
& & \\
$a$ is a constant and is $4 \times 10^{-4} \mathrm{~cm}^{2} \mathrm{~s}^{-1}$. & $\mathrm{D}=1.69 \times 10^{-4}\left(-17.48 \mathrm{P}^{3}+7.39 \mathrm{P}^{2}-2.89 \mathrm{P}+2.15\right)$ \\
M is a constant and is 0.82. & $\mathrm{P}^{4} \mathrm{P}^{2}$ & $\mathrm{P}_{\mathrm{c}}$ is the critical porosity and is 0.76.
\end{tabular}

By combining the results obtained from the constant and variable overlap methods, it is possible to see the change in $I_{s c}$ and $V_{o c}$ as a function of porosity. Through this, a porosity for the maximum $I_{s c}$ and $V_{o c}$ can be found and, thus, so can the point of maximum power output. $I_{s c}$ peaks at a porosity of 0.41 and $V_{o c}$ continues to rise gradually after $0.41{ }^{[54]}$ Therefore, a porosity of around 0.41 should be maintained to achieve maximum power.

\subsubsection{Nanowires}

\subsubsection{Electron Transport}

Electron transport in nanoparticle DSSCs, such as $\mathrm{TiO}_{2}$ has been theorised to occur either by a series of "hopping" steps between trap states (see Glossary) on neighboring particles, ${ }^{[58]}$ or via diffusion within extended states slowed down by trapping/detrapping events. ${ }^{[25]}$ An electron has been estimated to cross between $10^{3}$ to $10^{6}$ particles while traveling through the nanoparticle electron conductor. ${ }^{[25]}$ Electrons within a nanoparticle have high mobility, but the disorder of the nanoparticle network slows down the 
transport of an electron through the bulk film. Thus, the trap-limited diffusion is slow and limits carrier collection efficiency, particularly at lower energies corresponding to red wavelengths. ${ }^{[59]}$ Electron hopping in the nanoparticle network is efficient, but there is a link between electron transport and recombination, meaning that any improvement in the transport rate also increases the rate of recombination, and so the solar cell performance remains unchanged. ${ }^{[25]}$ This link is hyphothesised to be a result of the nanoparticle morphology, ${ }^{[25]}$ which can result in recombination percentages as high as $40 \%$, limiting efficiency to about $45 \%$. ${ }^{[36]}$ Thus, nanowire morphologies are potentially favourable, as there is little electron hopping necessary.

$\mathrm{ZnO}$ has nearly the same band gap $(3.3 \mathrm{eV})$ and electron affinity as $\mathrm{TiO}_{2}{ }^{[25]}$ and there has been an abundance of research in making $\mathrm{ZnO}$ nanowires (compared to that for $\mathrm{TiO}_{2}$ nanowires). Thus, the use of $\mathrm{ZnO}$ nanowires is currently being investigated as an alternative to $\mathrm{TiO}_{2}$ nanoparticle films. A dense array of oriented, single-crystal $\mathrm{ZnO}$ nanowires offers a highly-ordered photoanode of continuous, crystalline media for the electrons to travel through. The $\mathrm{ZnO}$ nanowires have an electron diffusivity of 0.05 to $0.5 \mathrm{~cm}^{2} \mathrm{~s}^{-1}$, which is several hundred times larger than that for $\mathrm{TiO}_{2}$ or $\mathrm{ZnO}$ nanoparticle films. ${ }^{[59]}$ When looking at equivalent surface areas, the currents are higher for the nanowire cells than for $\mathrm{ZnO}$ nanoparticle cells, and the current densities are the same as those of $\mathrm{TiO}_{2}$ nanoparticle cells. ${ }^{[59]}$ Nanowires improve electron transport, as they provide a direct path from the point of photogeneration to the conducting substrate. Every electron injected is directly connected to the substrate with a minimal number of interfaces and grain boundaries in between. ${ }^{[1]}$

The nanowires do not need to be ordered or perfectly perpendicular to the substrate, as randomly nucleated and coiled wires would still carry the injected electron to the anode. ${ }^{[25]}$ But perpendicular, untangled wires are favoured, as there is less chance of an electron having to cross a boundary. Perpendicular wires would also increase the donor to acceptor interfacial area and create electron transport pathways toward the negative electrode that possess very high electron mobility. Higher length-to-diameter ratios are also preferred, as they increase external quantum efficiency. ${ }^{[25]}$

\subsubsection{Surface Area}

Growing nanowires and making electron-conductor morphologies with "dendrite-like" branched structures leads to an enhancement in surface area compared to a nanoparticle film. ${ }^{[25]}$ A greater surface area is favourable, as it also increases the surface area of an applied dye, absorbed onto the electron 
conductor. The largest increase in surface area occurs between the formation of first and second generation growth (see Figure 4). ${ }^{[25]}$

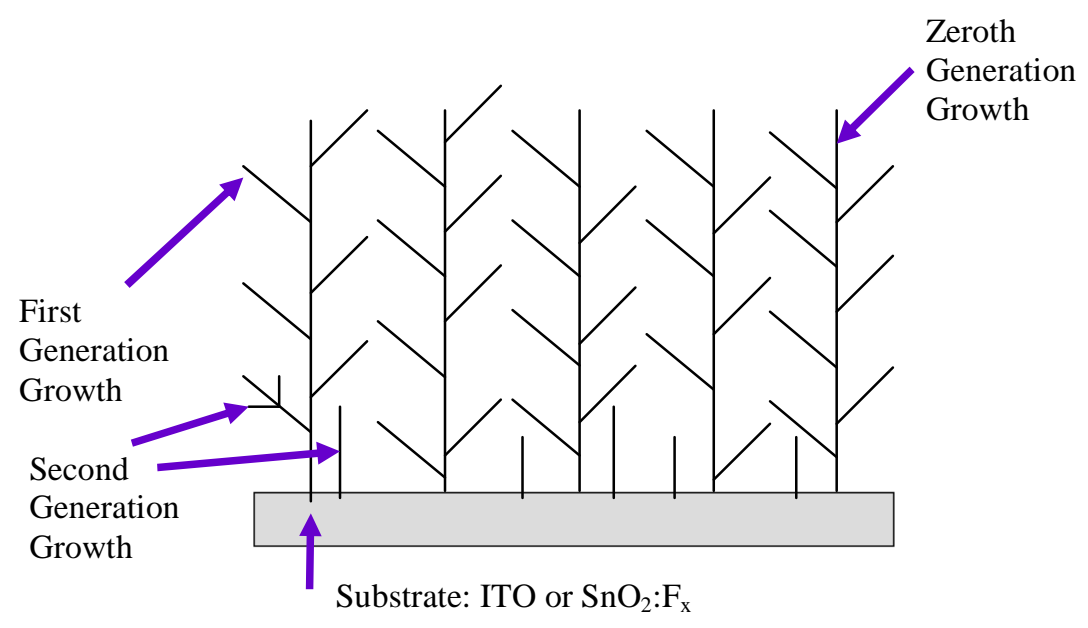

Figure 4. Nanowire Growth.

Thus far, the $I_{s c}, V_{o c}$ and overall efficiencies obtained for nanowire cells ${ }^{[25,27,50,59]}$ are relatively low when compared to $\mathrm{TiO}_{2}$ nanoparticle film cells. This is due to low light harvesting by the nanowire cells. DSSCs made from 10 to $15 \mu \mathrm{m}$ thick nanoparticle films usually absorb over $80 \%$ of incident light and have dye coverages of over $7.3 \times 10^{-8} \mathrm{~mol} / \mathrm{cm}^{2}$, compared to $9 \%$ and $9.0 \times 10^{-9} \mathrm{~mol} / \mathrm{cm}^{2}$ for nanowire-based solar cells. ${ }^{[25]}$ The surface area for the nanowires is limited by the growth method used. Growing longer and thinner nanowires with increased nucleation density should increase the surface area and would be more ideal. ${ }^{[25]}$ It is hypothesised that this could raise the current density to around $410 \mathrm{~mA} / \mathrm{cm}^{2} \cdot{ }^{[25]}$ An increase in the density of the nanowires could increase the effective path length of light, increasing absorbance of red wavelengths, where the dye's absorption is low. ${ }^{[25]}$

\subsection{Hole-Conducting Material}

The positively charged "holes" that are created as part of an electron/hole pair flow in the opposite direction to the electrons via a neighbouring bound electron moving into an existing hole, leaving a new hole behind (above, Figure 1(c)). The holes eventually pass into a hole conductor, the sensitiser is reduced and the circuit completed when the hole-conducting material is reduced by an electron that has traveled through the external circuit. The hole conductor can be liquid or solid, inorganic or organic. The photoexcited dye molecules are then regenerated by reduction. 
For efficiency, recombination of the generated electron/hole pair should be limited. This is particularly important at high light intensities, when large currents are forced through the cell. ${ }^{[60]}$ As sensitisers generate stable electron/hole pairs, it is desirable that the hole conductor not be capable of being photoactivated in the visible region, so as to maximise the sensitiser's light uptake in this region. Whether the electron and hole recombine in the dye sensitiser depends on the kinetics of the hole conductor. $^{[17]}$ The iodide ion has favourable kinetic properties, as its rate of reducing the sensitiser is greater than the rate of recombination for a typical sensitiser. Additionally, recapture of the electron by triiodide from the sensitiser is kinetically unfavourable. Because of the efficiency of the iodide/triiodide couple, most DSSCs use iodide as their reducing agent. For efficient hole transport to occur, the hole conductor must be well interpenetrated within the electron conductor's lattice. ${ }^{[3]}$ The redox properties of the hole conductor must also be fully reversible, which is fulfilled by the iodide/triiodide couple. ${ }^{[15]}$

Recombination can be prevented by use of an electrolyte additive, such as 4-tert-butylpyridine ${ }^{[52,61]}$ or pyridine ${ }^{[62]}$, as these adsorb onto the electron conductor surface, preventing the triiodide ion from interacting with it. Pyridine is also favourable as its low viscosity means that its addition does not decrease $I_{s c}$ noticeably. ${ }^{[63]}$

\subsubsection{Liquid Electrolytes}

Traditionally, in DSSC cells, liquid electrolytes (predominantly based on the iodide/triiodide redox couple) are used in an organic solvent (usually acetonitrile). ${ }^{[17]}$ The iodide donates an electron to the "hole" in the dye preventing recombination with the electron, ${ }^{[11]}$ reducing the sensitiser and becoming oxidised to triiodide. Iodide is subsequently regenerated by the reduction of triiodide ion at the counterelectrode with the circuit completion.

The iodide/triiodide couple is particularly favourable due to the largely irreversible nature of the iodide to triiodide transition; electrons are readily donated by iodide, but the reverse reaction is kinetically unfavourable. ${ }^{[64]}$ Substitution of the iodide/triiodide couple with other redox couples, such as ferrocenes, $(\mathrm{SCN})_{2} / \mathrm{SCN}^{[65]}$ or $\mathrm{Br}^{3-} / \mathrm{Br}^{-}$, does not show any improvement over the iodide couple. ${ }^{[12]}$ Hara et al. ${ }^{[20]}$ compared the $\mathrm{I}^{-} / \mathrm{I}_{3}{ }^{-}$couple to the $\mathrm{Br}^{-} / \mathrm{Br}_{2}$ couple. They found that the $V_{o c}$ was higher in the bromide system, which was attributed to $\mathrm{Br}^{-} / \mathrm{Br}_{2}$ having a redox potential of $0.65 \mathrm{~V}$ (vs. NHE), $0.25 \mathrm{~V}$ more positive than the potential of the iodide/triiodide couple. A smaller $I_{s c}$ was observed for the $\mathrm{Br}^{-}$ $/ \mathrm{Br}_{2}$, attributed to ineffective electron transfer from the $\mathrm{Br}^{-}$to the $\mathrm{HOMO}$ of the sensitiser due to a small energy gap between the $\mathrm{Br}^{-} / \mathrm{Br}_{2}$ redox potential and the $\mathrm{HOMO}$ of the sensitiser. 
Table 2. Hara et al.'s Results for Iodide Compared to Bromide in Acetonitrile with $\mathrm{TiO}_{2} \mathrm{DSSCs}^{[20]}$

\begin{tabular}{lcc}
\hline & $I_{s c}\left(\mathrm{~mA} / \mathrm{cm}^{2}\right)$ & $V_{o c}(\mathrm{mV})$ \\
\hline LiI & 3.42 & 0.52 \\
$\mathrm{LiBr}$ & 1.33 & 0.82 \\
\hline
\end{tabular}

Many polypyridyl cobalt(II/III) complexes have been tested and shown to potentially compete with the iodide/triiodide couple at low light intensity. ${ }^{[66]}$ However, mass transport limitations under full sunlight cause power efficiencies to drop below $4 \%$. ${ }^{[66]}$

Liquid, inorganic electrolytes have proven to be problematic because of their instability/degradation and propensity to evaporate under standard conditions. The volatile organic solvents used with the electrolytes require that the cells be completely sealed off from air. ${ }^{[3]}$ The liquid iodide/triiodide system is prone to photobleaching and makes the process of producing cells difficult because they have to be electrically connected, but chemically separated, preferably on a single substrate. ${ }^{[18]}$ Furthermore, the iodide/triiodide couple can be corrosive towards many electrode materials, such as platinum. ${ }^{[3]}$ All the DSSCs made that have achieved power conversion efficiencies of over $10 \%$ (the point at which they become economically viable) are not commercially viable because of their poor long term stability brought on by the liquid iodide/triiodide couple. ${ }^{[3,19]}$

Replacement of the liquid electrolyte with a solid-state analog (ionic solid ${ }^{[67]}$ or conducting polymer $^{[6 \text {, }}$ $15,18,19]$ ) increases stability. Recent research has looked at using a solid or gel hole acceptor, ${ }^{[6,38]}$ in which electronic, rather than ionic transport prevails. ${ }^{[10]}$ Ionic liquids have also been heavily researched. However, these have all proven to be less efficient than the liquid iodide/triiodide couple, and none have reached power conversion efficiencies over $10 \%$. For example: gel, organic and ionic polymer systems usually achieve power conversion efficiencies of 6,4 and $8 \%$ respectively. ${ }^{[3]}$ These lower efficiencies have been attributed to lower hole mobility, less favourable electron-transfer kinetics, and poor contact between these hole conductors and the electron-conducting material. ${ }^{[3]}$

\subsubsection{Electrolyte Concentration}

Hara et al. ${ }^{[20]}$ found that increasing the concentration of the $\mathrm{I}^{-}$source (from 0.007 to $3 \mathrm{~mol} / \mathrm{dm}^{3}$ ), while keeping the $\mathrm{I}_{2}$ source constant (at $0.03 \mathrm{~mol} / \mathrm{dm}^{3}$ ) affects both $I_{s c}$ and $V_{o c}$. On increasing concentrations of $\mathrm{I}^{-}, I_{s c}$ increases up to a certain point as there is an increase in conductivity of the electrolyte caused 
by both the $\mathrm{I}^{-}$and its cation. ${ }^{[20]}$ This infers that, up to the maximum $I_{s c}$, the rate determining step is the diffusion of the $\mathrm{I}^{-}$from the bulk and into the mesoporous oxide layer, and diffusion of the $\mathrm{I}_{3}{ }^{-}$from the oxide layer to the counter electrode. As the $\mathrm{I}^{-}$concentration increases beyond the maximum point, $I_{s c}$ decreases because of an increase in viscosity of the solution, which decreases ion mobility. ${ }^{[20]} V_{o c}$ also increases with greater $\mathrm{I}^{-}$concentration up to a maximum before decreasing. ${ }^{[20]}$ This was deemed to be due to kinetics.

According to equation 1 , as the concentration of $\mathrm{I}^{-}$increases, the concentration of $\mathrm{I}_{3}{ }^{-}$is also raised. This in turn pushes the reaction in equation 2 forward, enhancing the back electron transfer process, decreasing $V_{o c}{ }^{[20]}$ Thus, it is actually the $\mathrm{I}_{3}{ }^{-}$concentration that affects the cell performance. ${ }^{[52]}$

\section{Equation 1.}

$$
\mathrm{I}^{-}+\mathrm{I}_{2} \leftrightarrow \mathrm{I}_{3}^{-}
$$

Equation 2.

$$
\mathrm{I}_{3}{ }^{-}+2 \mathrm{e}_{\mathrm{cb}}{ }^{-} \leftrightarrow 3 \mathrm{I}^{-}
$$

$\mathrm{e}_{\mathrm{cb}}$ is the carrier (electron) density on the conduction band of the electron conductor

Hara et al. ${ }^{[20]}$ also looked at the effects of keeping the concentration of the $\mathrm{I}^{-}$source constant (at 0.3 $\left.\mathrm{mol} / \mathrm{dm}^{3}\right)$, while changing the $\mathrm{I}_{2}$ source $\left(0.005\right.$ to $\left.1 \mathrm{~mol} / \mathrm{dm}^{3}\right) . I_{s c}$ reached a maximum at $0.015 \mathrm{~mol} / \mathrm{dm}^{3}$ before decreasing with increasing $\mathrm{I}_{2}$ concentration. This was said to be due to increasing viscosity. $V_{o c}$ decreased as $I_{2}$ concentration increased because of enhanced back electron transfer. ${ }^{[20,68]}$

\subsubsection{Counter Cation Effect}

Liu et al. ${ }^{[68]}$ have reported the effects of the counter cation to the iodide on the overall cell performance. Having tested a variety of cations, they found that $V_{o c}$ is proportional to the ionic cation radius. This is because the cation species is absorbed onto the surface of the sensitised $\mathrm{TiO}_{2}$ resulting in a potential drop in the Helmholtz layer, the extent of which is dependent on the radius of the adsorbed cation and which increases as the radius decreases (Figure 5). The cation adsorbs onto the outside of the Helmholtz layer and does not affect the sensitiser/TiO ${ }_{2}$ interface. ${ }^{[69]}$ The potential drop caused by small cations results in a positive shift in the conduction band of the $\mathrm{TiO}_{2}$, the level of which again depends on the cation and its size. This positive shift of the $\mathrm{TiO}_{2}$ creates a larger energy gap between the excited state of the sensitiser and the conduction band (LUMO) of the $\mathrm{TiO}_{2}$, thus creating a larger photocurrent. $V_{o c}$ is dependant on the energy gap between the electron-conducting materials' 
conduction band (quasi-Fermi level) ${ }^{[52]}$ and the iodide/triiodide redox potential. A positive shift of the electron conductor's conductance band caused by small cations results in a smaller energy gap between the electron-conducting materials' conduction band and the iodide/triiodide redox potential, thus a smaller $V_{o c}$ is observed. Therefore, the cation affects the interfacial energy of the sensitised electron conductor. $^{[68]}$

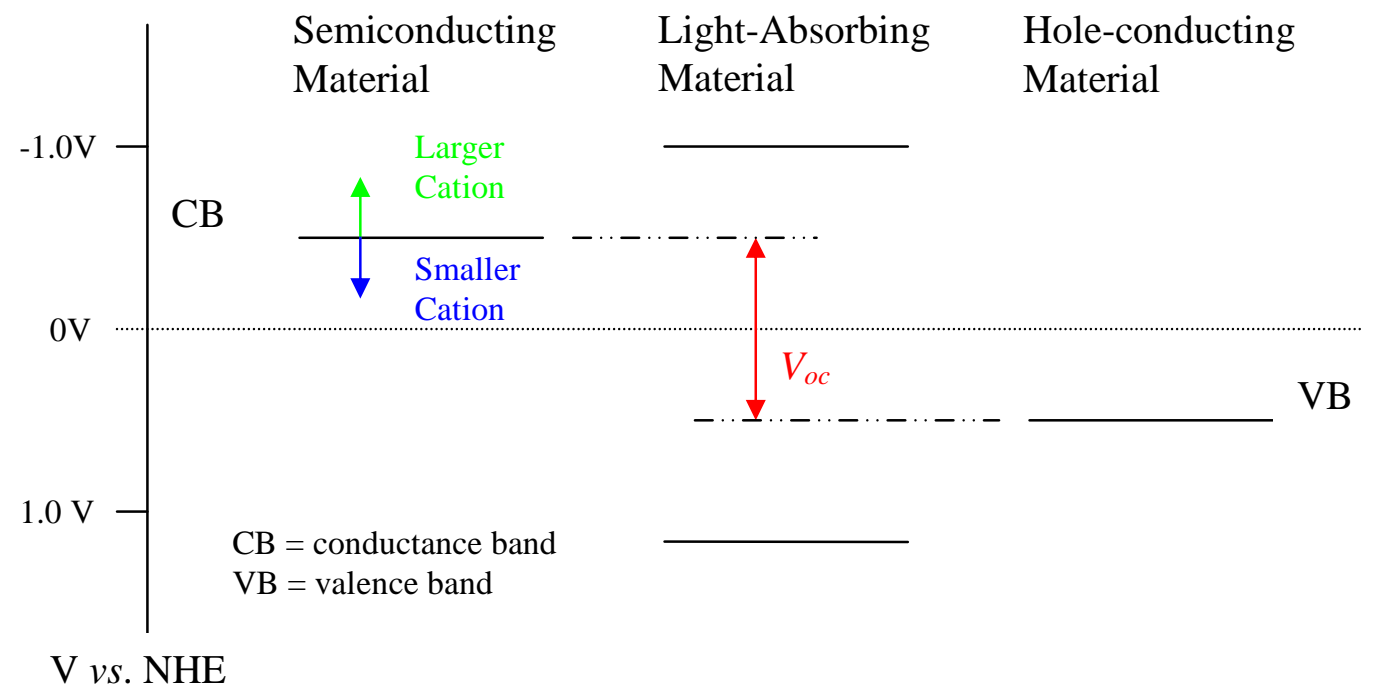

Figure 5. Effect of Cation on the Interfacial Energy of a Sensitised Electron-conducting Material.

Small, high charge density cations on the electron conductor's surface create deep traps sites, which prevent efficient transfer of electrons in the electron-conducting material. ${ }^{[52]}$ This results in an increase of recombination and lower $V_{o c}$, as the triiodide interacts more with the electron conductor's surface, which is made more positive by the interactions with the cation. Thus, the electron lifetime in the electron-conducting material is shortened. ${ }^{[70]}$

For example, Liu et al. ${ }^{[68]}$ studied $\mathrm{Li}^{+}, \mathrm{Na}^{+}, \mathrm{K}^{+}, \mathrm{Rb}^{+}$and $\mathrm{Cs}^{+}$iodides, and found that $\mathrm{Li}^{+}$gave the largest $I_{s c}$ and lowest $V_{o c}$. Of these cations, $\mathrm{Li}^{+}$has the smallest ionic radius, and as such leads to the largest potential drop in the Helmholtz layer, resulting in the largest positive shift of the electron conductor's conduction band. Thus, it has the largest gap between the sensitiser's excited state and the electron conductor's conduction band, leading to a high $I_{s c}$, but it has a smaller gap between the electron conductor's conduction band and the iodide/triiodide's redox potential, resulting in a smaller $V_{o c}$.

Hara et al. ${ }^{[20]}$ investigated LiI and $\operatorname{Pr}_{4} \mathrm{NI}$ as hole conducting electrolytes in mercurochrome sensitised $\mathrm{TiO}_{2}$ DSSCs (Table 3). Their results concur well with Liu et al.'s, ${ }^{[68]}$ as do Fredin et al.'s ${ }^{[70]}$ results. 
Table 3. Effect of Cations on Cell Performance in Acetonitrile (Hara et al.) with $\mathrm{TiO}_{2} \mathrm{DSSCs}^{\left[{ }^{[20]}\right.}$

\begin{tabular}{lcc}
\hline & $I_{s c}\left(\mathrm{~mA} / \mathrm{cm}^{2}\right)$ & $V_{o c}(\mathrm{~V})$ \\
\hline LiI & 3.42 & 0.52 \\
$\operatorname{Pr}_{4} \mathrm{NI}$ & 2.10 & 0.86 \\
\hline
\end{tabular}

Larger cations are less able to interact with the electron-conducting surface; their size causing decreased mobility between the gaps in the sensitiser coating. ${ }^{[68]}$ On irradiation, electron injection into the electron conductor's conductance band from the sensitiser raises its energy level, shifting it to more negative values, resulting in a larger $V_{o c}{ }^{[68]}$ A negative shift in the conduction band lowers the driving force for injection of electrons from the sensitiser, resulting in low injection efficiencies. ${ }^{[68]}$ Thus, $I_{s c}$ decreases as the cation radius increases. Furthermore, $I_{s c}$ is also affected by diffusion effects. Usually, the larger the cation, the less solvated it is and the more able it is to interact with iodide or triiodide, hindering their mobility. ${ }^{[68]}$ It is important to note that changes in ion mobility do not affect $V_{o c}$ greatly due to a logarithmic (rather than linear) relationship between the two.

Additionally, large cations with low charge density assist in smooth electron transfer in the electronconducting material by forming charge pairs to maintain a neutral interface, but allowing the negative charge to move through the electron conductor. ${ }^{[52]}$ Therefore, high $V_{o c}$ values are achieved, as there is less electron trapping and a longer electron lifetime. ${ }^{[70]}$ However, large cations result in the decrease of diffusion of the triiodide ion from the mesoporous oxide to the counter electrode, decreasing $I_{s c}{ }^{\text {[71] }}$ Paulsson et al. ${ }^{[72]}$ found that trap distribution is only marginally affected by the electrolyte cation, and more dependent on the bulk electron conductor.

As well as cation size, the strength of absorption onto the sensitised $\mathrm{TiO}_{2}$ surface also affects the $I_{s c}$ and $V_{o c}$, as the stronger the adsorption, the greater the electrostatic effect on the Helmholtz layer ${ }^{[69]}$ Hence, of two cations of comparable sizes, the cation with the stronger absorption will cause a greater potential drop in the Helmholtz layer, decreasing $V_{o c}$ and increasing $I_{s c}$. Larger cations also tend to improve the reductive capabilities of the iodide ion. ${ }^{[71]}$

Wang et al. ${ }^{[69]}$ looked at the dependence of $I_{s c}$ on the charge/radius ratio, which is effectively the charge density of a system, and found that the $I_{s c}$ increases as the ratio is raised. It does so linearly up to $1.5 \times 10 \mathrm{~nm}^{-1}$, after which the $I_{s c}$ continues to rise, but only slightly. This is in agreement with work 
done by Kelly et al., ${ }^{[73]}$ who found that electron injection from the sensitiser to the electron-conducting material increases with rising charge/radius ratio.

\subsubsection{Solvent Effects}

The effect of the solvent is dependent on its donor number (see Glossary). ${ }^{[60]}$ This is because the donor number affects which of the iodide species will predominantly exist in solution. Kebede et al. ${ }^{[60]}$ found that if a solvent has a low donor number, such as in Lewis acids like 1,2-dichloroethane, most of the $\mathrm{I}^{-}$ will exist as $I_{2}$ in the electrolyte by itself (not in a DSSC), ${ }^{[60]}$ as the solvent will not donate electron density readily, whereas, in a solvent with a high donor number, the majority of the $\mathrm{I}^{-}$will exist as $\mathrm{I}_{3}{ }^{-}$in the electrolyte. ${ }^{[60]}$ Kebede et al.'s findings appear to contradict those of other research groups, ${ }^{[20,61,63]}$ who have found that the higher donating ability results in a lower dark current, which is associated with a lower $\mathrm{I}_{3}{ }^{-}$content (Equations 1 and 2). Wu et al. ${ }^{[63]}$ proposed the following mechanism, based on the presumption that a higher donating ability results in a higher level of coupling with the oxidised $\mathrm{I}_{3}^{-}$:

\section{Equation 3.}

$$
\text { Solvent }+\mathrm{I}_{3}^{-} \rightarrow \text { Solvent- } \mathrm{I}_{3}^{-} \leftrightarrow \text { Solvent- } \mathrm{I}_{2}+\mathrm{I}^{-}
$$

Interestingly, Wu et al. ${ }^{[63]}$ discuss the findings of Kebede et al. ${ }^{[60]}$ but do not mention the inconsistency. It is possible that the differences are due to Kebede et al.'s data being from investigations of the electrolyte alone.

It has been found that larger $V_{o c}$ tend to be achieved with more basic (high donor number, DN) solvents, ${ }^{[20,61,63]}$ such as dimethylsulfoxide $(\mathrm{DN}=29.8),{ }^{[20,60]} \mathrm{N}, \mathrm{N}$-dimethylformamide $(\mathrm{DN}=26.6)^{[20 \text {, }}$ ${ }^{60]} \mathrm{N}$-methyl-2-pyrolidone $(\mathrm{DN}=27.3) .{ }^{[20,61]} V_{o c}$ increases are due to negative shifts of the electron conductor's conduction band by the basic solvents, which is more dominant as the solvent becomes more electron donating. ${ }^{[20,61,63]}$ The dark current at the electron-conductor-electrolyte junction is suppressed by the solvent molecules blocking access to the electron conductor's surface. ${ }^{[20,61]}$ However the basic nature of the solvents also causes sensitiser desorption from the electron-conducting material, which is not observed for the nitrile solvents. ${ }^{[20,61,63]}$

Lower $I_{s c}$ values are observed for high donor solvents because there is a decrease in the driving force for electron injection from the sensitiser to the electron conductor. ${ }^{[20,61,63]}$ Additionally, the high donor solvents tend to have greater viscosities, hindering ion mobility. ${ }^{[61]}$ For example, whereas the low 
donor number acetonitrile has a viscosity of $0.33 \mathrm{cP}$, the viscosity of the high donor dimethysulfoxide is $2.00 \mathrm{cP} .{ }^{[61]}$ The consequence of this is that larger $I_{s c}$ values are achieved in nitrile (low DN and viscosity) containing solvents, such as acetonitrile $(\mathrm{DN}=14.1)^{[60]}$ and methoxyacetonitrile $(\mathrm{DN}=$ 14.6). ${ }^{[20,63]}$ However, as a result of their lower donor numbers, lower $V_{o c}$ values are observed for nitrile-containing solvents. ${ }^{[20,61]}$

In alcoholic solvents, such as methanol $(\mathrm{DN}=19)^{[60]}$ and ethanol $(\mathrm{DN}=20)^{[60]}$, low $I_{s c}$ and high $V_{o c}$ values are observed. ${ }^{[20]}$ Hara et $a l .{ }^{[20]}$ observed that $V_{o c}$ was unstable in alcoholic solvents and decreased rapidly on constant irradiation. Dye desorption also occurred in alcoholic solvents.

Fukui et al. ${ }^{[61]}$ tested a mixture of a high donor solvent (dimethylsulfoxide, N,N-dimethylformamide, $\mathrm{N}$-methyl-2-pyrolidone and tetrahydrofuran) with acetonitrile to try and increase the $V_{o c}$ whilst maintaining a high $I_{s c}$. It was found that for the first three listed solvents, $V_{o c}$ and $I_{s c}$ increased with increasing donor number, thus indicating that dark current was suppressed, but the conduction band of the electron conductor was negative shifted, increasing the band gap. For the mixture with tetrohydrofuran, $I_{s c}$ remained quite constant with increasing donor number, while $V_{o c}$ increased. Hence, in the mixture with tetrahydrofuran, dark current was suppressed and there was no shift in the electron conductor's conduction band.

The donating ability of the solvent is related to the partial charge on the solvent. The greater the partial charge, the greater the $V_{o c}$ but the lower the $I_{s c}{ }^{\left[{ }^{61]}\right.}$ A large partial charge allows for a greater interaction between the solvent and electron conductor's surface, blocking the surface from $\mathrm{I}_{3}{ }^{-}$, but negatively shifting the electron conductor's conduction band, decreasing the driving force for electron injection from the sensitiser, thus decreasing $I_{s c}{ }^{[61]}$ For example, tetrahydrofuran does not have a great partial charge, so its interaction with electron conductor blocks the surface from the $\mathrm{I}_{3}{ }^{-}$, but does not affect the electron conductor's conduction band. ${ }^{[61]}$

The presence of water in a $\mathrm{TiO}_{2}$ DSSC with an acetonitrile solvent has been shown to increase $V_{o c}$ and decrease $I_{s c}{ }^{[68]}$ This change is not due to a negative shift in the conduction band of the $\mathrm{TiO}_{2}$, as it has also been shown that water causes a positive shift to the $\mathrm{TiO}_{2}$ conduction band. ${ }^{[74]}$ It was thus proposed that it was due to a decrease in back electron transfer. ${ }^{[68]}$ Water binds very strongly to the $\mathrm{TiO}_{2}$ surface, preventing the triiodide ion from interacting with the surface and recombining. Therefore, the blocking effect of the water is greater than the band shift effect. ${ }^{[68]}$ The decrease in $I_{s c}$ on water exposure was hypothesised to be because of hydrolysis or weakening of the $\mathrm{TiO}_{2}$-dye linkage, photosubstitution of 
the dye-ligands or a change in the adsorption properties of the dye as a consequence of polarity change. ${ }^{[68]}$ After exposure to water, the $V_{o c}$ cannot be restored to its prior value, whereas the $I_{s c}$ can. ${ }^{[68]}$

\subsubsection{Ionic Liquids}

The use of low viscosity, high volatility solvents inhibits the commercialisation of solar cells, because of the possible evaporation and degradation of the electrolyte. ${ }^{[6,19]}$ Sealing of the cells becomes problematic when cells are scaled up. ${ }^{[75]}$ Recently, room temperature molten salts or ionic liquids based on the imidazolium cation have been investigated as potential solvents, as they show favourable stability, ${ }^{[11]}$ are non-combustible, ${ }^{[2]}$ are able to form gels (with polymers, ${ }^{[76]}$ silica, ${ }^{[77]}$ small molecular weight gelators, ${ }^{[78,79]}$ carbon nanotubes, ${ }^{[80]}$ and even sub-micrometre anatase ${ }^{[81]}$ ) have a wide electrochemical window, ${ }^{[82]}$ and are considered to be more environmentally friendly than organic solvated electrolytes. ${ }^{[45]}$ Furthermore, ionic liquids only consist of ions, are high viscosity liquids over a wide range of temperatures (viscosity being proportional to the temperature) and show high ionic conductivities. $^{[2]}$ This high conductivity is attributed to the ability to promote the formation of polyiodides (for example: $\mathrm{I}_{3}^{-}, \mathrm{I}_{5}^{-}, \mathrm{I}_{7}^{-} \ldots$ ). ${ }^{[83]}$

Because of the favourable kinetics of the iodide/triiodide couple, most ionic liquids that have been tested are still based on this redox couple. Usually, rather than the source of iodide being one that requires solvation, a 1,3-dialkyimidazolium-based ionic liquid is used which has an iodide as its counter ion. Molecular iodine is added to the ionic liquid. 1,3-dialkyimidazolium ionic liquids are favoured because they are able to solvate ionic and molecular species despite being miscible in organic solvents and other ionic liquids. ${ }^{[45]}$ The ability to change the two alkyl groups quite readily also makes the 1,3-dialkyimidazolium ionic liquids attractive. ${ }^{[45]}$ For example: 1-methyl-3-hexyl imidazolium iodide, 1-methyl-3-propyl imidazolium iodide, 1,2-dimethyl-3-propyl imidazolium iodide. ${ }^{[12]} 1$-allyl-3ethylimidazolium iodide and 1-allyl-3-propylimidazolium iodide. ${ }^{[46]}$ Binary systems of two ionic liquids have also been investigated. ${ }^{[75,84,85]}$ A possible problem with imidazolium-based iodide salts is their hygroscopic nature, requiring that they be kept in absolutely dry conditions. ${ }^{[12]}$

\subsubsection{Diffusion}

As advantageous as it is to have a low volatile hole conductor, ionic liquids have a major drawback in their high viscosity, which is problematic for mass transport of ions, particularly at high light intensities. ${ }^{[72,86]}$ The viscosity of a typical ionic liquid is one hundred times greater than acetonitrile, 
and 30 time greater than water. ${ }^{[76]}$ The diffusion of the triiodide ion is usually the limiting factor in most DSSCs, with the diffusion coefficients of molten electrolytes being about an order of magnitude lower than those of liquid electrolytes. ${ }^{[72,76]}$ Consequently, they show decreases in efficiency as the cell thickness increases, as diffusion of iodide/triiodide from ionic liquids is much slower than that from a liquid system due to their high viscosity. ${ }^{[53]}$ Consequently, a thin nanocrystalline $\mathrm{TiO}_{2}$ film is required to achieve a high conversion efficiency. In almost all cases, the power conversion efficiency of liquid electrolyte cells is higher than that of ionic liquid cells. ${ }^{[53]}$

Kawano et al. ${ }^{[76,86,87]}$ have shown that high efficiency ionic liquid cells are achievable despite their high viscosity, because of the different diffusion mechanisms inherent to their systems. There are two contributions to the charge transport of the iodide/triiodide redox couple, physical diffusion and exchange-reaction based diffusion. It has been shown that exchange-reaction based diffusion (Equation 4) dominates at high iodide/triiodide concentrations, ${ }^{[87]}$ and is the reason why ionic liquids show relatively fast hole transport despite their extremely high viscosity. ${ }^{[86]}$ This exchange-reaction based diffusion has been shown to only occur in ionic liquids and not liquid electrolytes, because the redox couple in ionic liquids exists in a strong ionic field. ${ }^{[86]}$ Conversely, physical diffusion takes place in both ionic liquids and liquid electrolytes. ${ }^{[86]}$ A DSSC made up of an ionic liquid was shown to be capable to achieving photocurrent of up to $90 \%$ of equivalent liquid electrolyte cells. ${ }^{[76]}$

\section{Equation 4. Exchange-Reaction Diffusion.}

$$
\mathrm{I}_{3}^{-}+\mathrm{I}^{-} \leftrightarrow \mathrm{I}^{-}+\mathrm{I}_{3}^{-}
$$

Cell performance with ionic liquids is greatly dependent on the structure of the imidazolium cation and its ionic conductivity and viscosity. However, it has been found that the $I_{s c}$ does not quantitatively depend on these factors; other consequences, such as the ionic liquid's effect on the potential gap between the semiconductor's Fermi level and the redox couple's equilibrium potential, also contribute to the resulting $I_{s c}{ }^{[76]}$ Furthermore, as in liquid electrolyte cells, 4-tert-butylpyridine ${ }^{[52,61]}$ and LiI can also be added to ionic liquid cells to enhance $V_{o c}$ by preventing back electron transfer. ${ }^{[76]}$

\subsubsection{Dependence on the Iodide/Triiodide Couple.}

The dependence on the iodide triiodide couple is significant in ionic liquid-based DSSCs ${ }^{[45]}$ and, historically, it has proven to be the most efficient redox couple for DSSCs (as discussed above, Part I 
1.4.1). Even systems not using the imidazolium cation often use the iodide/triiodide couple; for example, trialkyl sulphonium iodide. ${ }^{[72]}$ Often, when imidazolium anions are not iodine based, they are used in conjunction with an iodide source. For example, 1-ethyl-3-methylimidazolium dicyanamide was used with iodide (dicyanamide was found to cause instability on illumination), ${ }^{.75]} 1$-ethyl-3methylimidazolium thiocyanate was used in a binary system with 1-propyl-3-methylimidazolium iodide, which achieved over $7 \%$ power conversion efficiency, ${ }^{[75]}$ and 1-ethyl-3-methylimidazolium tetracyanoborate was used in a binary ionic liquid system with 1-propyl-3-methylimidazolium iodide and this proved to be a stable low viscosity system with a reasonably high conversion efficiency of 7 $\% .{ }^{[84]}$

Interhalogen ionic liquids such as 1,3-dialkyimidazolium with $\mathrm{IBr}_{2}^{-}$or $\mathrm{I}_{2} \mathrm{Br}^{-}$(trihalide anions) have also been tested because of their lower viscosities, with some success. ${ }^{[82]}$ Compared to the iodide/triiodide couple, such interhalogen systems are favourable in their higher flexibility in redox species, due to equilibria between different anions in solution. ${ }^{[82]}$

\subsection{3 "Soggy Sand" Electrolytes}

Electrolytes consisting of (usually) liquid, non-aqueous salt solutions may also be used in conjunction with solid insulating particles. ${ }^{[88]}$ Such electrolytes have been termed soggy sand electrolytes, rather than just being solid-liquid composites, as their co-use is not merely additive, but synergistic with respect to overall conductivity. ${ }^{[88]}$ These electrolytes achieve this effect through the adsorption of the cations or anions from the salt onto the surface of the particles used, leaving the opposite ions free for moving charges. ${ }^{[88,89]}$ This is known as "interfacial conductivity enhancement".

Battacharyya et el. ${ }^{[88]}$ reported the conductivity of electrolytes comprising of $\mathrm{LiClO}_{4}$ with one of three different oxides $\left(\mathrm{TiO}_{2}, \mathrm{SiO}_{2}\right.$ or $\left.\mathrm{Al}_{2} \mathrm{O}_{3}\right)$, in either methanol or THF. They did this for increasing volume contents of the oxides. In methanol, the conductivities were found to be very dependant on the surface $\mathrm{pH}$ of the oxides. The more acidic the surface of the oxide was $\left(\mathrm{SiO}_{2}>\mathrm{TiO}_{2}>\mathrm{Al}_{2} \mathrm{O}_{3}\right)$, the higher the conductivity achieved. Thus, it was theorised that the anions were adsorbed onto the surfaces of the oxides, enhancing the $\mathrm{Li}^{+}$concentration in the space charge layer surrounding the oxide particles. The surface charge density was found to affect the conductivity in proportion to the oxide content. When there were only small amounts of $\mathrm{SiO}_{2}$ present, the double layer repulsion on its surface resulted in poor conductivities. Conversely, $\mathrm{Al}_{2} \mathrm{O}_{3}$ has a lower charge density, which gave rise to a smooth increase in conductivity as the oxide content was enhanced. At some volume fraction, different for each 
oxide $\left(\sim 0.2, \mathrm{Al}_{2} \mathrm{O}_{3}>\mathrm{TiO}_{2}>\mathrm{SiO}_{2}\right)$, the conductivity peaked, before dropping off. This decrease was attributed to porosity effects, namely that pathways become blocked by "dry" oxide particles. In THF, there was a greater degree of ion association than in methanol, which was due to the much lower dielectric constant of THF (7.4 compared to 32.6).

With $\mathrm{TiO}_{2}$ DSSCs, stable electrolytes utilising LiI in different solvents ${ }^{[89,90]}$ and, in particular, their amalgam with solid insulating particles ( such as $\mathrm{SiO}_{2}$ ) have been reported. ${ }^{[77,89-91]}$ An et al. ${ }^{[90]}$ assessed the use of $\mathrm{LiI} / \mathrm{I}_{2}$ in ethanol with $\mathrm{SiO}_{2}$ nanoparticles, in a $\mathrm{TiO}_{2}$ DSSC. They achieved high power conversion efficiencies $\left(6.1 \%\right.$ ), almost identical to those achieved with the purely liquid $\mathrm{LiI} / \mathrm{I}_{2}$ ethanol electrolyte, despite the much higher viscosity of the $\mathrm{SiO}_{2}$-containing electrolyte. This was because the ionic conductivity of the $\mathrm{SiO}_{2}$-containing electrolyte was only $16 \%$ less than that of its liquid counterpart. The identical conversion efficiencies obtained were attributed to the fact that the $\mathrm{SiO}_{2}$ particles used were smaller than the pores of $\mathrm{TiO}_{2}$, such that the gel electrolytes could still penetrate the mesoporous network and achieve good contact with the semiconducting material. Furthermore, triiodide and iodide were able to move freely in the channels of the $\mathrm{SiO}_{2}$ network.

Wang et al. ${ }^{\left[{ }^{[9]}\right.}$ reported similar results with LiI, 3-hydroxypropionitrile (HPN) and $\mathrm{SiO}_{2}$. It was found that the use of micro-sized $\mathrm{SiO}_{2}$ increased the ionic conductivity of the liquid electrolyte and improved the overall cell current, potential and conversion efficiency of a $\mathrm{TiO}_{2} \mathrm{DSSC}_{\text {. Using nano-sized particles }}$ enhanced this even further. In the LiI-HPN system, the $\mathrm{Li}^{+}$is coordinated to $\mathrm{O}$ and $\mathrm{N}$, thus it is the iodide that is the mobile charge carrier. Seeing as $\mathrm{SiO}_{2}$ has a relatively acidic surface and should interact with the anions, the conductivity should be reduced. Hence, the increase in conductivity was attributed to the formation of amorphous regions at the $\mathrm{SiO}_{2} / \mathrm{LiI}-\mathrm{HPN}$ interface, where the iodide ions had more mobility than when in the bulk. A power conversion efficiency of $5.4 \%$ was obtained.

Work has also been done on the use of nanoparticle $\mathrm{SiO}_{2}$, as a gelator, with ionic liquids, specifically 1methyl-3-propylimidazolium iodide. ${ }^{[77,92-95]}$ Wang et al. ${ }^{[77]}$ reported that, though the $\mathrm{SiO}_{2}$ electrolyte had a viscosity far greater than the equivalent liquid electrolyte, the two electrolytes showed almost identical steady-state voltammograms, indicating that triiodide and iodide were able to move freely in the channels of the silica network. The results obtained in a $\mathrm{TiO}_{2} \mathrm{DSSC}$ were very promising, achieving power conversion efficiencies up to $7 \%$, matching those achieved by their liquid counterparts. Berginc et al. ${ }^{[93]}$ reported increased charge transportation in their $\mathrm{SiO}_{2}$-containing ionic liquid electrolytes, which they found increased cell efficiency by up to $20 \%$. 


\section{Solid-State DSSCs}

DSSCs may be fabricated from solid-state dye-sensitised heterojunctions, consisting of two solid semiconducting materials, one $n$-type and the other $p$-type. Where liquid DSSCs are molecular electronic devices, which operate on the basis of chemical conversion on the molecular scale, solidstate DSSCs act as sensitised $p$ - $n$ heterojunctions with the behaviour of semiconductors. ${ }^{[96]}$ When the dye is excited, the electron flows into the $n$-type semiconductor, which has a wide band gap and is inorganic. The hole travels to the $p$-type semiconductor, which can be inorganic or organic. ${ }^{[17]}$

Having a solid-state hole conductor solves sealant issues, but poses inherent problems in achieving interpenetration into the electron conductor's surface. For good conduction, the sensitiser must be in good contact with both of the semiconducting materials, which is problematic in solids. ${ }^{[17]}$ This is particularly true as the cell gets thicker than $2 \mu \mathrm{m}$, as the filling of the pores gets more difficult as the cell thickness increases. ${ }^{[96]}$

The $p$-type material has the same requirements as all hole conductors, in that it must be transparent to the visible spectrum (have a wide band gap), there must be a way to deposit it onto the electronconducting material without degrading it, and its valence band must correlate well with the sensitiser's HOMO. ${ }^{[97]}$ In a typical solid cell, the $n$-type electron conductor would be the $\mathrm{TiO}_{2}$ and the $p$-type hole conductor would be, for example, $\mathrm{CuI}^{[97]}$ or $\mathrm{CuSCN} .{ }^{[28]} \mathrm{CuI}$ is a good choice because it has a wide band gap $(3.1 \mathrm{eV})$ and dissolves in organic solvents to allow deposition. ${ }^{[97]}$ Its valence band also aligns nicely, being located just above the HOMO of the N3 dye. ${ }^{[96]}$ With CuI power conversion efficiencies of $3 \%$ have been observed in a ruthenium sensitised $\mathrm{TiO}_{2}$ cell. ${ }^{[98]}$ The thiocyanate groups on the ruthenium dye bind well with the cuprous ion. ${ }^{[99]}$

CuSCN also has a wide band gap (3.6 eV) and good alignment of its valence band with the HOMO of the N3 dye. ${ }^{[100]}$ However, it is not very soluble in most organic solvents, and is only fully soluble in dipropyl sulfide, which is poisonous. A power conversion efficiency of $2 \%$ was obtained from a ruthenium sensitised $\mathrm{TiO}_{2}$ cell using $\mathrm{CuSCN} .{ }^{[100]}$

Spirobisfluororene-connected acrylamine (spiro OMeTAD) has also been tested. ${ }^{[101,102]}$ It is an amorphous material, so is able to form inside the electron conductor's pores, where it connects well 
with the sensitiser monolayer, reducing the oxidised sensitiser rapidly. ${ }^{[103]} \mathrm{A} \mathrm{TiO}_{2}$ DSSC using spiro OMeTAD has been reported to have a power conversion efficiency of over $5 \% .{ }^{[104]}$

A major hindrance of solid-state DSSCs, particularly from $\mathrm{CuI}$ and $\mathrm{CuSCN}$, is that they suffer from a large amount of recombination. ${ }^{[96]}$ This is due to the large interfacial area between the two semiconductors, compared to the liquid system where the triiodide ion is able to diffuse away from the electron conductor. ${ }^{[96]}$ This problem would not be so prevalent if a complete monolayer of the sensitiser could be deposited on the $n$-type electron conductor, however this is not possible due to the large size of the sensitiser and repulsion among the molecules. ${ }^{[96]}$ Thus, undesirable contacts between the two semiconductors are unavoidable in solid-state DSSCs.

Recombination at the sensitiser is not usually a problem, as reduction of the oxidised sensitiser happens faster than recapture of the ejected electron. ${ }^{[103]}$

\section{$3 \quad$ Photogalvanic Solar Cells}

Photogalvanic solar cells are based on photoelectrochemical processes, which involve charge separation by physical movement of species. ${ }^{[105]}$ In photogalvanic cells, current and voltage changes result from photochemically generated changes in the relative concentrations of reactants in a solution phase oxidation-reduction couple. These cells have not been researched as extensively since the discovery of the newer DSSCs, as they have, historically, tended to require the use of toxic and expensive materials, such as cadmium-selenide and iron-ruthenium. ${ }^{[37,105]}$

\subsection{Semiconductor Photogalvanic Cells}

In some ways, this form of photgalvanic cell resembles a DSSC without a sensitiser, except that the semiconducting material is on the electrode furthest from the light and the electrons do not travel through the semiconducting material but through the electrolyte solution. When light hits such as cell, it penetrates all the way through the cell to the back photoactive $p$-semiconductor electrode. Electrons are released, reducing the electrolyte. These reduced ions flow to the opposite electrode, where they are oxidised back to their original form by releasing the gained electrons. The hole created moves to the opposite electrode, where it meets released electrons flowing through an external circuit (usable by an 
external load). The circuit is completed by returning electrons to the semiconducting material (Figure 6). $n$-Type semiconductor photogalvanic cells are equally viable.

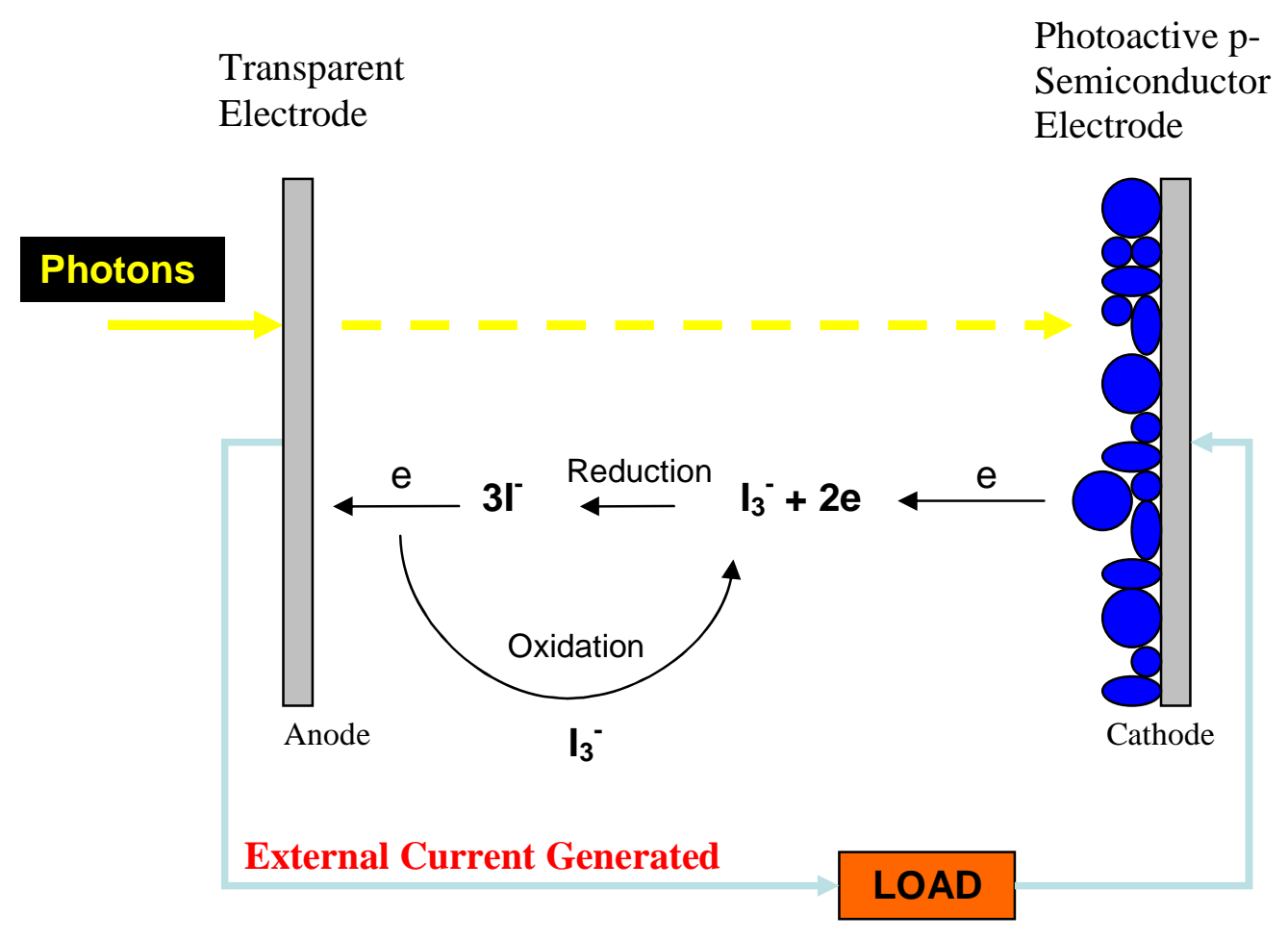

Figure 6. $p$-type Semiconductor Photogalvanic Cell.

Photogalvanic cells necessitate the separation of the photogenerated electron and hole and their respective associated ionic species. ${ }^{[106]}$ This is also true for DSSCs, except that the use of a dye sensitiser means that the electron and hole are physically separated, making recombination of the initially excited species less likely. In photogalvanic cells, physical separation is problematic, as it requires both fast electron injection into the electron transporter and fast electron and hole transfers. This transfer involves physical migration of ions. ${ }^{[106]}$ Hence, only thin films are viable for photogalvanic cells, ${ }^{[106]}$ and the illuminated electrode must be selective. ${ }^{[105]}$

Semiconductor photogalvanic cells tend to accumulate charge, resulting in oxidation or reduction of the semiconducting material instead of the desired redox reaction. ${ }^{[105]}$ Additionally, the greatest results of $12 \%$ power conversion efficiency have been achieved with single crystals, which is unhelpful for large scale application. ${ }^{[105]}$ An example is the GaAs cell with a selenide-polyselenide redox system. ${ }^{[107]}$ 


\subsection{Thin-Layer-Electrolyte Photogalvanic Cells}

In these photogalvanic cells no semiconducting material is employed, but merely a photoactive redox electrolyte, ${ }^{[106]}$ or a mixture of two redox couples, one of which can be photoactivated. Examples of the latter are iron-thionine systems, ${ }^{[105,108]}$ and iron-ruthenium systems. ${ }^{[37,105]}$ These photochemical systems have two redox reactions, each of which occurs at separate electrodes. ${ }^{[109]}$ Thus, physical separation of the activated species is again required.

To control which reactions take place at each electrode, the front electrode is transparent while the back electrode becomes the "dark electrode", which the light does not interact with (Figure 7). ${ }^{[105]}$ Because the cell medium is a fluid, the reverse reaction occurs rapidly in the absence of an inhibitor, or promoter of the desired reaction. ${ }^{[108]}$ Thus, for an efficient photogalvanic cell one of the electrodes must show selectivity for one of the redox reactions ${ }^{[37]}$ and the electrode kinetics of the two electrodes must be different. ${ }^{[108]}$

\section{Photons}

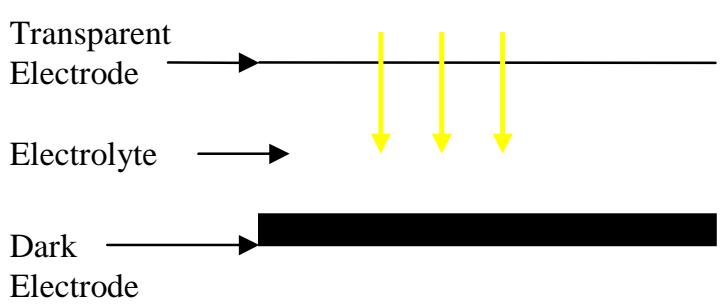

Figure 7. Thin-Layer-Electrolyte Photogalvanic Cell.

An example of a single electrolyte photogalvanic cell is the iodide/triiodide couple, as the triiodide ion is capable of being photoactivated (Figure 8). ${ }^{[106]}$ It was found that the iodide/triiodide couple has an efficiency peak at approximately $430 \mathrm{~nm}$, which tails off to a negligible value at $710 \mathrm{~nm}$. Unlike DSSCs or semiconducting photogalvanic cells, the distance between electrodes does not greatly affect cell performance. Stevenson et al. ${ }^{[106]}$ reported less than a $10 \%$ change in power output upon changing the cell width from 1 to $4 \mathrm{~mm}$.

That the iodide/triiodide couple can act in a photogalvanic capacity is interesting if one considers that one of the requirements of a DSSC hole conductor is that it be transparent to the visible spectrum so as 
not to affect the light uptake by the sensitiser in DSSCs (Part I 1.2). No publications on DSSCs refer to the fact that the iodide/triiodide couple can itself be photoactivated or the effects this may have on the overall cell performance. This is possibly because the currents and potentials obtained from these photogalvanic cells tend to be low compared to those achieved by published DSSCs.

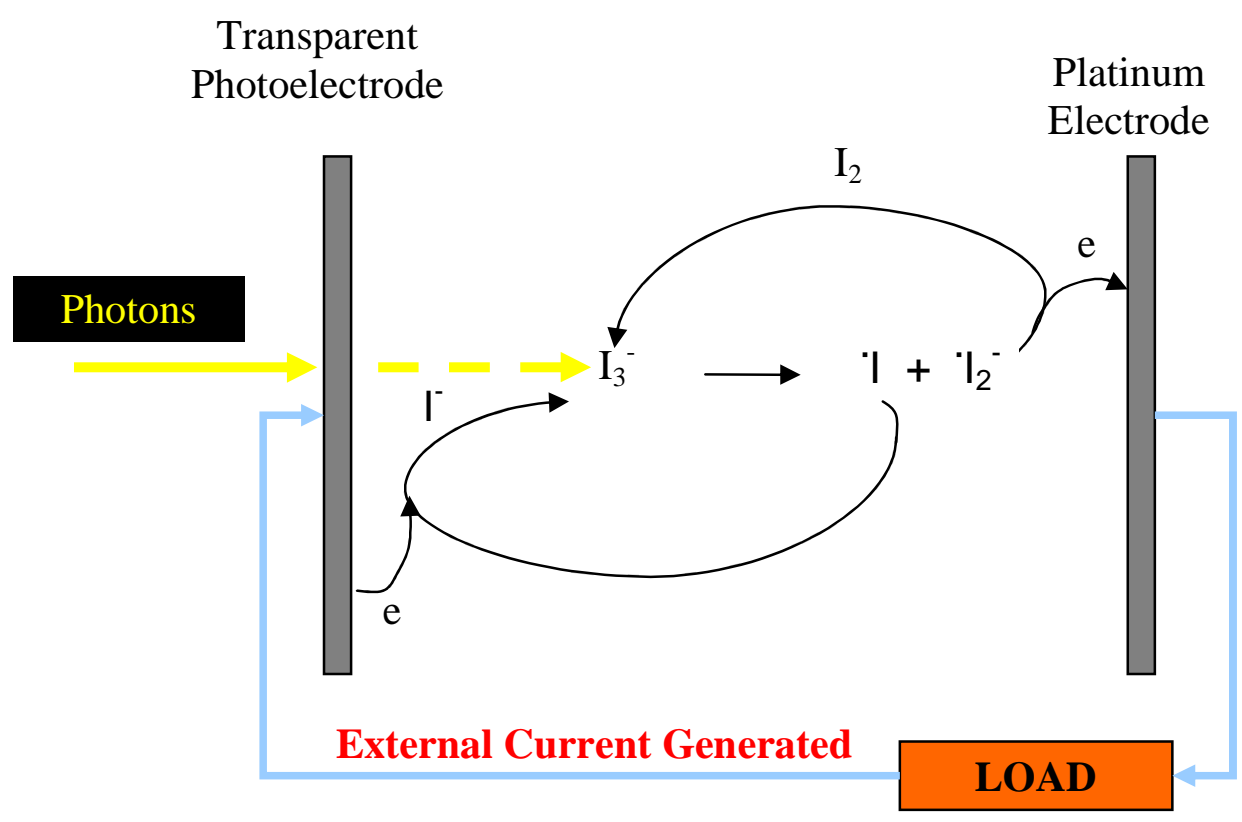

Figure 8. Thin-Layer-Electrolyte Iodide/Triiodide Photogalvanic Cell.

Some research has been carried out with dye sensitising these electrolyte photogalvanic cells, however this is minimal compared with research on DSSCs. ${ }^{[105]}$ Furthermore, the dyes investigated were not used to enhance the spectral range of the semiconducting material, were unstable and merely used to prevent corrosion of the electrodes. 


\section{$4 \quad$ Nanostructured Calcium Silicate}

Crystalline calcium silicate (wollastonite) does not have semiconductive properties and is not photoactive. However, a non-crystalline nanostructured calcium silicate (NCaSil) developed by researchers at Victoria University of Wellington has been found to have photoactive and semiconductive properties when in slurry form. ${ }^{[10-113]}$ Nanostructured calcium silicate particles are made up of nano-sized plates, 2 to $10 \mathrm{~nm}$ thick and approximately $100 \mathrm{~nm}$ wide, which come together to form a microstructure with a gypsum desert-rose appearance (Figure 9). ${ }^{[113]}$ These have meso- as well as nano-pores and a very high surface area of up to $600 \mathrm{~m}^{2} \mathrm{~g}^{-1} \cdot{ }^{[111]}$ Nanostructured calcium silicate also has a very low manufacturing cost, such that it could have commercial viability, even if it were unable to achieve power conversions as high as its $\mathrm{TiO}_{2}$ analogues.

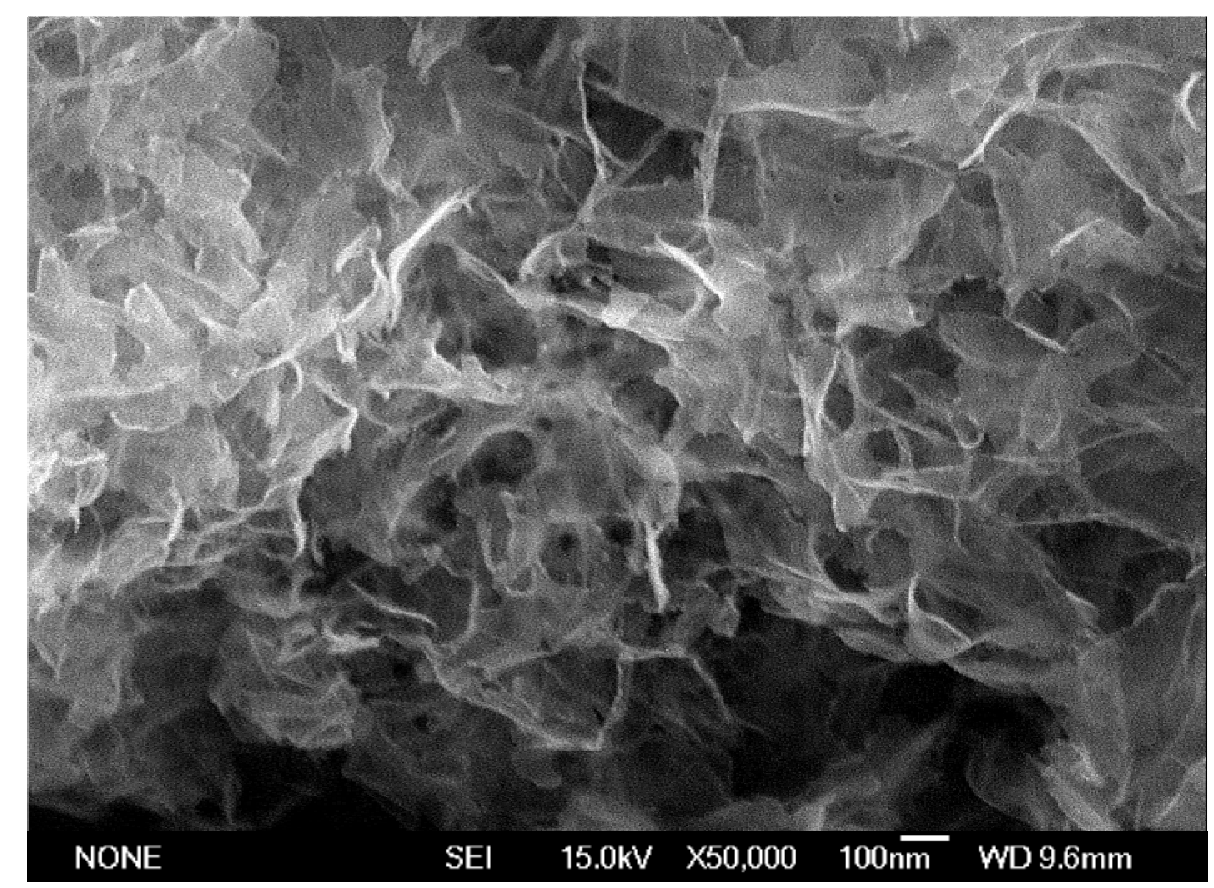

Figure 9. Reinforced Nanostructured Calcium Silicate (at $\times 50000$ magnification).

\subsection{Photoactive Properties}

At the low energy end of the UV-spectrum $(\lambda \leq 400 \mathrm{~nm})$, the most energy-rich visible light is found, at around $400 \mathrm{~nm}, \mathrm{E}=3.1 \mathrm{eV}$ ( $\mathrm{as} \mathrm{E}=\mathrm{hc} / \lambda$ ). Thus, it is preferable that the band gap of an electronconducting material be above $3 \mathrm{eV}$. This is so the semiconducting material does not compete with any sensitiser for light uptake and is not destabilised by visible light. In its dry form, NCaSil shows absorbance over the whole spectral range, peaking at $272 \mathrm{~nm}$ (Figure 10). ${ }^{[110]}$ 


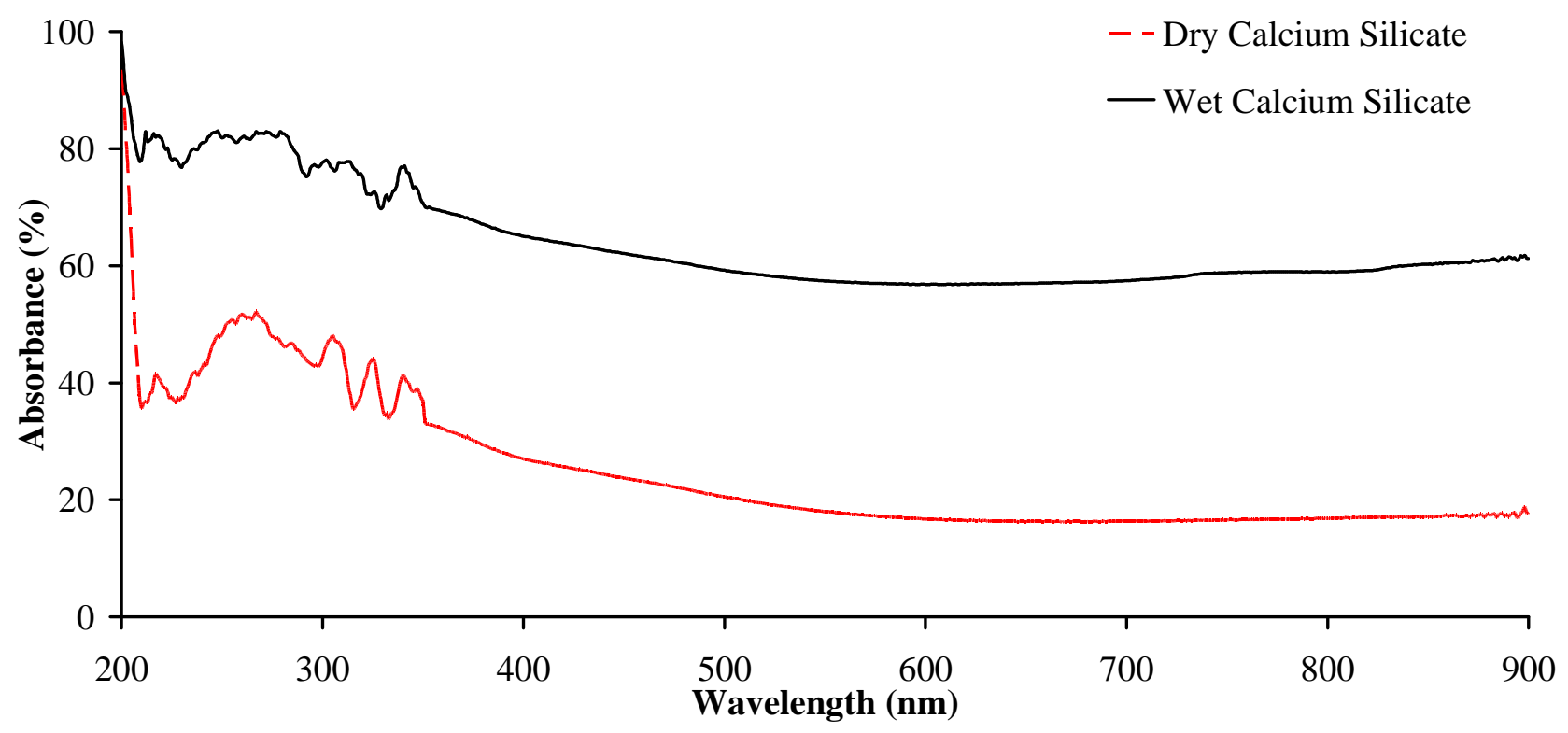

Figure 10. Absorbance of Dry and Wet Nanostructured Calcium Silicate. ${ }^{[110]}$

However, the material appears white (Table 4), scattering and reflecting most incident light. When wet, at the same concentration, the NCaSil shows the same spectral range, but has higher absorbances due to the reflective indices of water and the silicate being closer than those of silicate and air. This means the silicate appears more transparent, thus dispersing light more greatly and increasing the light path. ${ }^{[10]}$

Table 4. Optical Properties of Different Types of NCaSil (data from PhD thesis of Dr Andy McFarlane, School of Chemical and Physical Sciences, Victoria University of Wellington).

\begin{tabular}{lccccc}
\hline & $\mathrm{L}^{*}$ & $\mathrm{a}^{*}($ Red - & $\mathrm{b}^{*}($ Yellow & $457 \mathrm{~nm}$ & $577 \mathrm{~nm}$ \\
& $($ Luminescence) & $\begin{array}{c}\text { Green } \\
\text { Undertone) }\end{array}$ & $\begin{array}{c}\text { - Blue } \\
\text { Undertone) }\end{array}$ & $\begin{array}{c}\text { (TAPPI- } \\
\text { Brightness) }\end{array}$ & $\begin{array}{c}\text { (ISO- } \\
\text { Brightness) }\end{array}$ \\
\hline NCaSil & 98.83 & 0.11 & 0.04 & 96.80 & 97.13 \\
RNCaSil & 95.73 & -0.14 & -1.03 & 90.58 & 89.02 \\
EENCaSil & 95.72 & -0.72 & 2.74 & 85.97 & 89.85 \\
\hline
\end{tabular}

Luminescence is a form of cold body radiation.

The "undertone" relates to the light scattering ability of the material and how this varies as a function of wavelength, e.g., at the red-green or yellow-blue end of the visible spectrum.

TAPPI Brightness is the reflection at $457 \mathrm{~nm}$.

ISO-Brightness is the reflection at $577 \mathrm{~nm}$. 
The excitation spectrum of NCaSil peaks in the ultraviolet to blue region. The band gap has been estimated from the absorption and excitation spectra to be around $3.6 \mathrm{eV}$, making it a good candidate for use in solar cells, as it is not destabilised by visible light. By contrast, the band gaps of the iodide/triiodide electrolyte (from absorption data obtained by Kebede et al. ${ }^{[60]}$ ) are $2.48 \mathrm{eV}\left(\mathrm{I}_{2}\right), 3.44$ and $4.27 \mathrm{eV}\left(\mathrm{I}_{3}{ }^{-}\right)$, and $5.64 \mathrm{eV}\left(\mathrm{I}^{-}\right)$. Thus, $\mathrm{I}_{2}$ can be destabilised by visible light (likely resulting in photobleaching) and, furthermore, is able to compete with dye sensitisers for light uptake. This depends on the extinction coefficient of the $\mathrm{I}_{2}$, its concentration and the cell thickness.

No work has been done utilising NCaSil in a solar cell set up. It is not known whether, when used with the iodide/triiodide electrolyte, the NCaSil's photoactivty would dominate the cell's performance or if this would be dwarfed by the photogalvanic process of the iodide/triiodide couple. Furthermore, if the NCaSil's photoactivity does dominate the cell, it is not clear whether the NCaSil acts as a semiconducting material as in a DSSC or semiconductor photogalvanic cell.

\subsection{Semiconducting Properties}

When dried at temperatures above $300{ }^{\circ} \mathrm{C}$, NCaSil is only mildly semiconductive $\left(5 \times 10^{-9} \mathrm{Sm}^{-1}\right.$; the cut-off for semiconductivity is approximately $\left.10^{-10} \mathrm{Sm}^{-1}\right),{ }^{[110]}$ and is only sufficiently semiconductive in its slurry form. In water (10\% solid content, wt $\%$ ), conductivities of around $1.1 \times 10^{-1} \mathrm{Sm}^{-1}$ have been observed. ${ }^{[110]}$ This was possibly due to increasing ion mobility and the release of calcium and hydroxyl ions. Comparatively, values extrapolated from data obtained by Shedlovsky and Brown ${ }^{[114]}$ indicate the conductivity of $\mathrm{CaCl}_{2}$ electrolyte solution, of the same concentration of $\mathrm{Ca}^{2+}$ as in the NCaSil, would be closer to $1 \times 10^{3} \mathrm{Sm}^{-1}$.

It is not clear whether the silicate structure itself is semiconducting or if it is simply a polyelectrolyte. The residual conductivity observed of the dried calcium silicate was possibly due to loosely bound water on the silicate structure (even after heating at $300^{\circ} \mathrm{C}$ ), which allows some ion transport. ${ }^{[110]}$

\subsection{Surface Area Effects}

The high surface area afforded by the plate structure of NCaSil makes it a potentially good candidate for solar cell application from the perspective of maximum sensitiser surface. If only a monolayer of 
sensitiser were absorbed onto the surface of the semiconducting material, the greater the semiconductor surface area, the greater the dye surface and, theoretically, light uptake. The larger pores of around 200 $\mathrm{nm}$ in diameter also make NCaSil favourable for efficient diffusion of hole conductors. ${ }^{[13]}$ (Note: The pore size of NCaSil is by no means uniform. ${ }^{[115]}$ ) However, the inherently disordered nature associated with its high surface area is a potential disadvantage of calcium silicate, as higher ordered materials tend to transport electrons more efficiently. ${ }^{[28]}$

\subsection{Challenges Inherent to Nanostructured Calcium Silicate}

\subsubsection{Possible Stability Issues}

A significant issue with nanostructured calcium silicate is that it tends to lose some of its network structure when in liquid for extended periods of time. Reinforcement of the calcium silicate overcomes this to a large extent. Whether the calcium silicate can be used in cells for longer time periods is important for any practical application and as such long-term use is investigated in this project.

\subsubsection{Alkalinity}

Nanostructured calcium silicate creates a very different environment than $\mathrm{TiO}_{2}$, as it releases hydroxyl ions in water, resulting in an alkaline solution with $\mathrm{pH}$ values of approximately 10.5 to 11.5 . As a consequence, cells made up with the calcium silicate may remove the coatings (carbon, platinum or gold) off the back electrode, particularly in cells tested over long periods of time. Eventually, all the coating may be removed.

\subsubsection{Consistency of Nanostructured Calcium Silicate Formation}

The task of generating different batches of nanostructured calcium silicate showing consistent properties is challenging. Its amorphous and random structure means that slight differences in the methodology taken, such as mixing order, time of stirring and speed of addition and mixing, results in differences in particle size and surface areas. Great care has to be taken in keeping note of the exact methodology used to achieve repeatability to the level required for photovoltaic studies. 


\subsection{The Interaction of Nanostructured Calcium Silicate and Iodine}

It has been found that calcium silicate interacts with iodine in two different manners depending on the temperature. ${ }^{[115]}$ At temperatures below $32.5^{\circ} \mathrm{C}$, the calcium silicate acts as a container for iodine. The iodine is not incorporated into the structure, but merely enters its pores. ${ }^{[15]}$ This physical adsorbance is fully reversible, the rate of which is temperature dependent and is greater at 32 than $22^{\circ} \mathrm{C} .{ }^{[115]}$

At temperatures above $32.5{ }^{\circ} \mathrm{C}$, bonds are formed between the calcium silicate and iodine with the extent of bonding being dependent on the silicate structure. ${ }^{[115]}$ There are a limited number of uniform sites available for iodine interaction on the silicate structure, and the larger the surface area of the silicate, the more iodine can be taken up. ${ }^{[115]}$ Other sources of energy, such as friction and blending, have also been found to be effective in bonding iodine to calcium silicate. ${ }^{[115]}$ Such bonding is not fully reversible, even at temperatures above $600{ }^{\circ} \mathrm{C}$. The iodine is not fully removed until $720{ }^{\circ} \mathrm{C}$, the point at which the calcium silicate structure collapses and forms wollastonite, its thermodynamically favoured (but non-photoactive) form. ${ }^{[115]}$ X-ray Photoelectron Spectroscopy (XPS) indicates iodine interaction with calcium, oxygen and silicon, but the exact nature of the interaction has yet to be resolved. ${ }^{[15]}$

Such findings regarding the interaction of NCaSil and iodine are particularly important for the photovoltaic use of nanostructured calcium silicate, as most solar cells depend on the iodide/triiodide couple as their hole conductor. Long periods of illumination are likely to raise the temperature of the cell above $32.5^{\circ} \mathrm{C}$ or offer enough energy to the system for the calcium silicate to react with iodine. If calcium silicate is acting as a semiconductor, such an interaction may inhibit the photocurrent of calcium silicate cells, as the close contact of the electron conductor and hole conductor would likely result in recombination of an electron/hole pair. The use of a sensitiser could inhibit this interaction.

It is not clear how such an interaction would affect cell performance, particularly if the calcium silicate is not acting as a semiconductor, but merely supplying ions and a network in which the photogalvanic process of the triiodide ion could take place. It is possible that the interaction may not take place at all in the electrolyte solution. There may be insufficient $\mathrm{I}_{2}$ around, as it may all react with the $\mathrm{I}^{-}$to form $\mathrm{I}_{3}{ }^{-}$. 


\section{$5 \quad$ Measuring Cell Performance}

\subsection{Fill Factor}

From the plots of current and voltage the fill factor $(f f)$ of a sample can be measured. This is a measure of cell performance rather than cell efficiency, as it does not relate to the amount of energy entering the cell. It only looks at the cell output. Figure 11 shows the general curves obtained in a Graetzel type $\operatorname{DSSC}^{[9]}$ compared to curves obtained from a photogalvanic cell ${ }^{[106]}$.
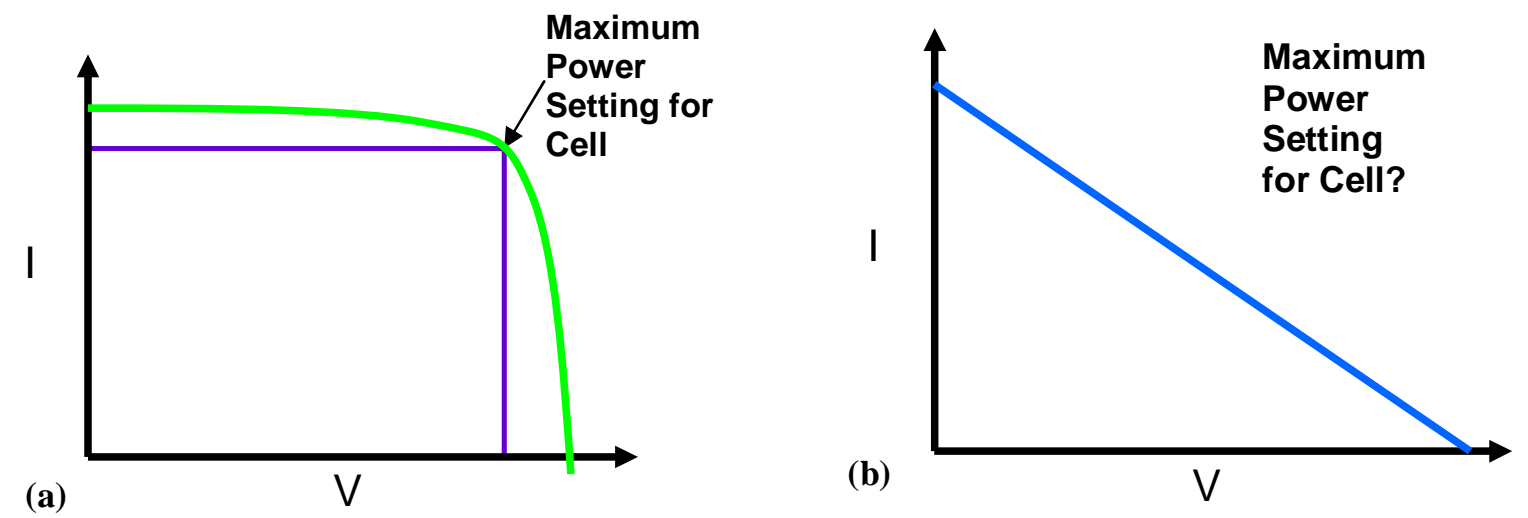

Figure 11. Current versus Voltage Plots for a (a) Graetzel Type DSSC and (b) Photogalvanic Cell.

The fill factor is the area within the point of maximum power $\left(V_{m p} \times I_{m p}\right)$, and is defined as a fraction of $V_{o c} \times I_{s c}$ (see equation 5). Thus, an ideal cell would have a rectangular curve, and would have a fill factor of 1. It can be seen in Figure 11 that the Graetzel type cell usually has a very rectangular curve, and cells often have fill factors over 0.85 . It can equally be observed that, though it is readily calculable for a Graetzel type cell, the linearity of the photogalvanic cell curve means that there is no obvious maximum power point and the fill factor is always around $0.25 .^{[106]}$

\section{Equation 5.}

$$
f f=\left(V_{m p} I_{m p}\right) /\left(V_{o c} I_{s c}\right)
$$

$V_{m p}$ is defined as the voltage at the maximum power.

$I_{m p}$ is defined as the current at the maximum power.

$\left(V_{m p} I_{m p}\right)$ is the point of maximum power output.

$V_{o c}$ is the voltage when there is no load under open-circuit conditions.

$I_{s c}$ is the maximum current through a load under short-circuit conditions. 
That the photogalvanic cells show ohmic resistance can be seen as a positive aspect, when one considers that this affords great flexibility in the conditions that the cell is able to have its maximum power setting in. By comparison, DSSCs have a nearly rectangular current over voltage curve, ${ }^{[28,116]}$ offering only a single point of operation. Though, assuming the same $I_{s c}$ and $V_{o c}$, the rectangular curves offer higher powers than the comparative linear curves for most voltages.

\subsection{Overall Power Conversion Efficiency}

The overall power conversion efficiency $\left(\eta_{g l o b a l}\right)$ measures how much energy is produced by the cell in comparison to the amount of light energy that reaches the sample. The values obtained for this calculation are always lower than those obtained for via the Incident Photon Current Efficiency (IPCE), and are the values usually quoted in papers for overall cell performance.

\section{Equation 6.}

$$
\eta_{g l o b a l}=\left(I_{s c} V_{o c} f f\right) / A_{c} I_{l}
$$

$I_{l}$ is defined as the light intensity of the incident light.

$A_{c}$ is defined as the surface area of the cell.

\subsection{Incident Photon-to-Current Conversion Efficiency}

The Incident Photon Current Efficiency (IPCE, $\eta$ ) differs from the calculations of the overall power conversion efficiency, as it measures how much of the incident light is converted into electron/hole pairs, by measuring the number of electrons generated, and does so at each specific wavelength. ${ }^{[10]}$ It depends on light absorption, electron injection and charge extraction under short circuit conditions. The IPCE is a measure of how efficient the light absorber is than of the whole cell as a unit. Values obtained for the IPCE of DSSCs are often high (over $90 \%$ ).

\section{Equation 7.}

$$
\mathrm{n}=\frac{\mathrm{N}_{\mathrm{el}}}{\mathrm{N}_{\mathrm{ph}}}=\frac{\mathrm{hc}}{\mathrm{e}} \times \frac{I_{s c}}{\mathrm{P}_{\text {light }} \lambda}
$$

$\mathrm{N}_{\mathrm{el}}$ is the number of detected electrons. $\quad \mathrm{e}$ is the elementary charge.

$\mathrm{N}_{\mathrm{p}}$ is the number of incident photons. $\quad \lambda$ is the wavelength of the incident light.

$\mathrm{h}$ is Planck's constant. $\quad I_{s c}$ is the measured short-circuit current optimised to a constant area.

$\mathrm{C}$ is the speed of light. $\quad \mathrm{P}_{\text {light }}$ is the incident light power area optimised to a constant area. 


\section{RESEARCH OBJECTIVES}

This project involves two objectives. The first investigates the intrinsic properties of nanostructured calcium silicate (NCaSil) when used in conjunction with the iodide/triiodide electrolyte. An assessment is made as to whether the NCaSil contributes to the photoactivity observed from such silicate-based cells. Where possible, the dominant photoactive components are ascertained and, if the NCaSil does act as the photoactive semiconducting material, it is important to discover whether it is doing so in the capacity of a DSSC or semiconductor photogalvanic cell. These issues are to be determined by testing NCaSil with the iodide/triiodide electrolyte within different parameters (for example, the effect of cell depth and width) and comparing results to the literature for DSSCs and photogalvanic cells. Additionally, results are compared to analogous tests of the electrolyte alone and attempts are made to ascertain whether the silicate backbone plays an integral part of the cell process.

If most of the activity observed is attributed to the iodide/triiodide electrolyte, an assessment is made as to whether NCaSil used with the iodide/triiodide acts as a soggy sand electrolyte. This is achieved through comparing the $\mathrm{NCaSil}$ and iodide/triiodide cells to $\mathrm{TiO}_{2}$ and $\mathrm{SiO}_{2}$ cells, tested under the same conditions, and comparing the results to the literature. Furthermore, the interaction of the iodide/triiodide electrolyte with NCaSil, particularly with long-term testing, will also be important towards this assessment.

Secondly, optimisation of the cell is undertaken in order to achieve as high a power output and light conversion as possible. This is to be attained by selectively changing different components of the cell make-up. The components to be varied include (not exclusively): the NCaSil itself (i.e., modified and doped NCaSils), solvent variation, NCaSil:solvent ratio, different electrolyte additive cations and content amount, cell width and depth, and the nature of the electrodes.

On many occasions both objectives are assessed simultaneously, as observing the effects of changing certain variables also elucidates the inherent properties of the nanostructured calcium silicate and its role when in a solar cell. Thus, a better understanding of the nanostructured calcium silicate's function when used in cells and the type of cells formed, as well as a reasonably optimised cell, is simultaneously sought throughout this project. 


\section{EXPERIMENTAL}

\section{Synthesis and Development of Nanostructured Calcium Silicate}

All forms of NCaSil were prepared according to the literature. ${ }^{[111,113]}$

\subsection{Batch Synthesis NCaSil}

NCaSil was formed by reacting sodium silicate with calcium hydroxide. ${ }^{[111,117]} \mathrm{Ca}(\mathrm{OH})_{2}(34.69 \mathrm{~g})$ was placed into a $1000 \mathrm{~cm}^{3}$ plastic beaker (silicate reacts with glass) with distilled water $(470 \mathrm{~g})$. The resulting slurry was mixed rigorously using an overhead stirrer. Concentrated $\mathrm{HCl}\left(24 \mathrm{~cm}^{3}, 33 \%\right)$ was gradually added, yielding a $\mathrm{pH}$ of 11.3 to 11.5 . The stirrer was positioned off-centre to achieve good mixing. Into a $1000 \mathrm{~cm}^{3}$ plastic beaker, sodium silicate (NaSil) solution was weighed $(87.8 \mathrm{~g}$, Orica, $28.7 \% \mathrm{SiO}_{2}$ ) and water was added to make up $500 \mathrm{~cm}^{3}$. The resulting solution was mixed well. The solutions were combined under intensive mixing and left stirring rapidly for five minutes, before the stirring speed was reduced and the solution left mixing. The typical NCaSil structure develops over several hours after the initial mixing. The NCaSil slurry was used for later preparations.

Water-washed (untreated, $\mathrm{Ca}_{12} \mathrm{O}_{12} \mathrm{Si}_{20} \mathrm{O}_{33} \cdot 4 \mathrm{CaCO}_{3} \cdot \mathrm{Na}_{2} \mathrm{O} \cdot 4 \mathrm{H}_{2} \mathrm{O}$ ) calcium silicate was obtained by filtering under vacuum and washing the filter cake extensively with at least $2000 \mathrm{~cm}^{3}$ of water. The washed filter cake was dried at $105^{\circ} \mathrm{C}$ for 48 hours.

On drying, untreated nanostructured calcium silicate exhibits a loss of surface area and pore volume due to the collapse of the microstructure caused by strong attractive forces between surface silanol and calcium groups and surface tension. ${ }^{[113]}$ Pretreatment of the calcium silicate before drying by (1) reinforcement; (2) the removal of some calcium and silanol groups ${ }^{\text {[113]; }}$ or (3) addition of a spacer compound and surface tension lowering agent, generally alleviates this collapse of the microstructure.

\subsection{Reinforcement of NCaSil}

In the reinforcement steps, sodium silicate was used as a reinforcing agent. Addition of this precipitated silica onto the plates, encapsulating and preventing the collapse of the structure and loss of the accessible surface area, resulting in reinforced nanostructured calcium silicate (RNCaSil, 
$\mathrm{Ca}_{6} \mathrm{O}_{6} \mathrm{Si}_{15} \mathrm{O}_{30} \cdot 2 \mathrm{CaCO}_{3} \cdot \mathrm{Na}_{2} \mathrm{O} \cdot 2 \mathrm{H}_{2} \mathrm{O}$ ). NaSil $\left(2.8 \mathrm{~g}\right.$ ) was added in distilled water (10 to $25 \mathrm{~cm}^{3}$ ) to every $50 \mathrm{~cm}^{3}$ of the slurry from 1.1. The resulting slurry was stirred for 1 minute, before $\mathrm{HCl}\left(5 \mathrm{~cm}^{3}, 2\right.$ $\mathrm{mol} / \mathrm{dm}^{3}$ ) was added drop-wise. The acid-treated mixture was stirred for 30 minutes, filtered under vacuum, washed extensively with water, and dried at $105^{\circ} \mathrm{C}$ for 48 hours.

Five batches of RNCaSil were made for the purposes of this project: JCL/1/53B, JCL/4/77A, JCL/5/07A, JCL/5/11A and JCL/5/37A, hereinafter called Batch \#1, 2, 3, 4 and 5, respectively, when differentiation is required.

\subsection{Acid Washed NCaSil}

Removal of calcium and silanol to form acid washed nanostructured calcium silicate (AWNCaSil, $\mathrm{Ca}_{2} \mathrm{O}_{2} \mathrm{Si}_{15} \mathrm{O}_{30} \cdot \mathrm{Na}_{2} \mathrm{O} \cdot 2 \mathrm{H}_{2} \mathrm{O}$ ) was achieved by washing with $\mathrm{HCl}$. Removing some of the surface functionality rendered the NCaSil plates less likely to interact with each other, increasing the stability of the NCaSil structure. In the first step of manufacturing AWNCaSil, $\mathrm{HCl}(33 \%)$ was dripped slowly into the slurry of calcium silicate from 1, until a $\mathrm{pH}$ of 7 was achieved. The final $\mathrm{pH}$ of 7 was maintained for 5 minutes without further addition of $\mathrm{HCl}$ to ensure completion. Then the treated silicate was filtered under vacuum, washed extensively with water and dried at $105{ }^{\circ} \mathrm{C}$ for 48 hours.

\subsection{Spacer-Compound Containing NCaSil}

2-Ethoxyethanol acted as a spacer compound between the silicate plates and lowered the surface tension of water, preventing collapse on drying. Silicates treated this way are referred to as ethoxyethanol treated NCaSil (EENCaSil, $\mathrm{Ca}_{12} \mathrm{O}_{12} \mathrm{Si}_{20} \mathrm{O}_{33} \cdot 4 \mathrm{CaCO}_{3} \cdot \mathrm{Na}_{2} \mathrm{O} \cdot 4 \mathrm{H}_{2} \mathrm{O}$ ). To make EENCaSil, the filter cake of the slurry from 1.1 was washed extensively with water on a Buchner filter. When there was only about $5 \mathrm{~mm}$ of water standing above the filter cake, 2-ethoxyethanol was added, the volume of which was approximately half the volume of the cake. The sample was dried at $105{ }^{\circ} \mathrm{C}$ for 48 hours.

\subsection{Continuous Method RNCaSil}

Two main types of reinforced calcium silicate were tested. The first was batch-processed reinforced calcium silicate, produced as outlined above (batch RNCaSil, referred to hereafter as RNCaSil). The second was a "continuous method" reinforced calcium silicate, in which $20 \mathrm{dm}^{3}$ batches of the starting 
materials were prepared and reacted in a small chamber, using a peristaltic pump to achieve a continuous flow of starting materials (Figure 12). The size of the reaction vessel allowed for better control over the stirring speed and consequently the particle size of the resulting $\mathrm{NCaSil} .{ }^{[111]}$ Continuous method RNCaSil (CM-RNCaSil) was prepared by Mr James Grinrod, of the School of Chemical and Physical Sciences, Victoria University of Wellington (JEG3-16B).

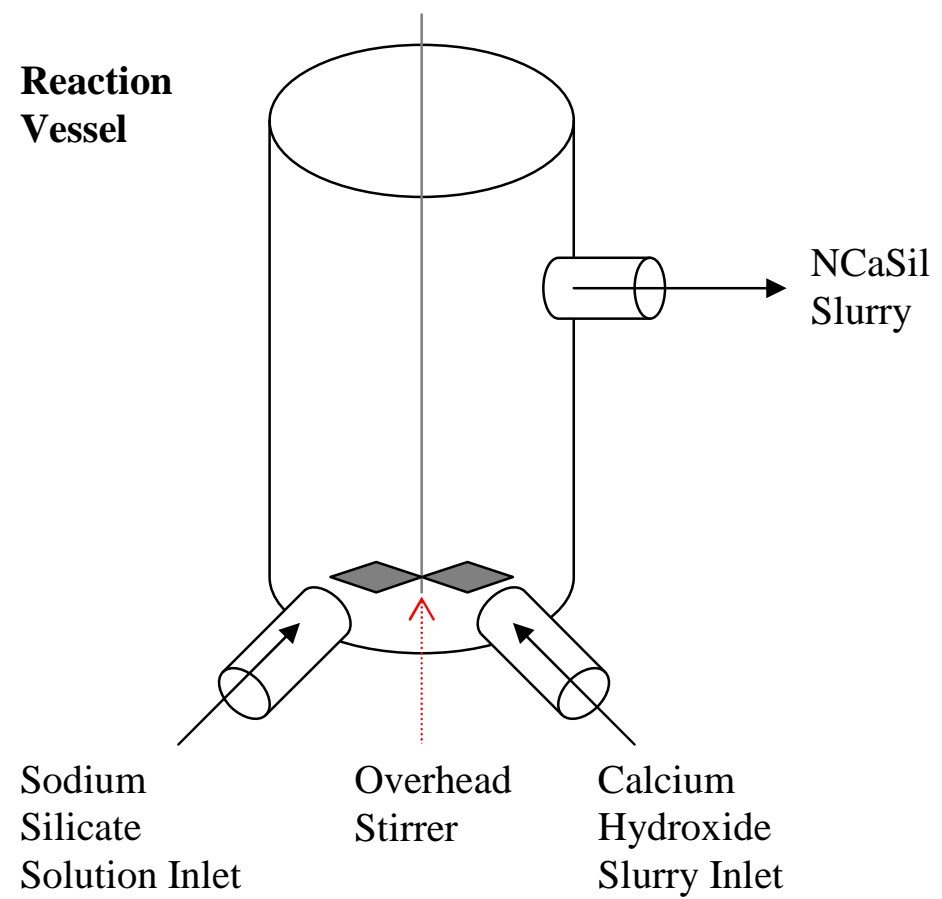

Figure 12. Continuous Method Set Up.

The sodium silicate and calcium hydroxide solutions were pumped into the reaction vessel at the same flow rate. The produced NCaSil continuously left the reaction vessel through an upper outlet. This CMNCaSil was reinforced by in a batch method to yield CM-RNCaSil.

\subsection{Other Treated NCaSils}

The treated NCaSils were prepared by Dr. Thomas Borrmann. V- and Ce-NCaSil (TB2004 and TB2005 respectively) by the addition of cerium chloride or sodium vanadate to the calcium hydroxide slurry prior to the reaction with sodium silicate. Similarly, Ru-containing NCaSil was prepared by addition of ruthenium trichloride (TBRu-EECaSil). I2 -treated EENCaSils (TB10/09/2007) was made by by reacting EENCaSil with iodine at $120^{\circ} \mathrm{C}$ for 48 hours. 


\section{$2 \quad$ Electrolytes Used}

The main electrolyte used $\left(0.588 \mathrm{~mol} / \mathrm{dm}^{3}\right.$ potassium iodide (Pure Science, $\left.99 \%\right), 0.096 \mathrm{~mol} / \mathrm{dm}^{3}$ iodine (Unilab, 99.5\%)) was prepared in de-ionised water ( $\mathrm{DI} \mathrm{H}_{2} \mathrm{O}$, purified by a Milli-Q Ultrapure Water System) or a 70:30 in weight mixture of an alternative solvent and $\mathrm{DI}_{2} \mathrm{O}$.

\subsection{Electrolyte Solvent}

Many different solvents were used to solvate the iodide/triiodide couple and tested with and without NCaSil. The iodine and iodide salt are insoluble in many organic solvents. Consequently, water/organic solvent mixtures were prepared in 30 to $70 \mathrm{wt} \%$ ratios (Table 5).

Table 5. Formulation of Iodide/Triiodide Electrolytes from Different Solvents.

\begin{tabular}{|c|c|c|c|c|c|c|c|}
\hline \multirow[b]{2}{*}{ Solvent } & \multicolumn{3}{|c|}{$\begin{array}{c}\mathrm{m}= \\
1.435 \mathrm{~g}\end{array}$} & \multirow{2}{*}{$\begin{array}{c}{[\mathrm{KI}]=0.588} \\
\mathrm{~mol} / \mathrm{dm}^{3}\end{array}$} & \multirow{2}{*}{$\begin{array}{l}\mathrm{Mr}(\mathrm{KI})= \\
166 \mathrm{~g} / \mathrm{mol} \\
\mathrm{m}(\mathrm{KI})(\mathrm{g})\end{array}$} & \multirow{2}{*}{$\begin{array}{c}{\left[\mathrm{I}_{2}\right]=0.096} \\
\mathrm{~mol} / \mathrm{dm}^{3}\end{array}$} & \multirow{2}{*}{$\begin{array}{c}\mathrm{Mr}\left(\mathrm{I}_{2}\right)= \\
253.8 \mathrm{~g} / \mathrm{mo} \\
\mathrm{m}\left(\mathrm{I}_{2}\right)(\mathrm{mg})\end{array}$} \\
\hline & $\begin{array}{l}\text { Density } \\
\left(\mathrm{g} / \mathrm{cm}^{3}\right)\end{array}$ & $\mathrm{V}\left(\mathrm{cm}^{3}\right)$ & $\begin{array}{c}\text { Total V } \\
\left(\mathrm{cm}^{3}\right)\end{array}$ & & & & \\
\hline Acetone & 0.785 & 1.83 & 2.44 & 1.44 & 0.239 & 0.235 & 59.5 \\
\hline Acetonitrile & 0.780 & 1.84 & 2.45 & 1.44 & 0.240 & 0.236 & 59.8 \\
\hline Ethanol & 0.785 & 1.83 & 2.44 & 1.44 & 0.239 & 0.235 & 59.5 \\
\hline $\begin{array}{l}\text { 2-Ethoxy } \\
\text { ethanol }\end{array}$ & 1.110 & 1.29 & 1.91 & 1.12 & 0.186 & 0.183 & 46.5 \\
\hline $\begin{array}{l}\text { Ethylene } \\
\text { Glycol }\end{array}$ & 0.930 & 1.54 & 2.16 & 1.27 & 0.211 & 0.207 & 52.6 \\
\hline Propan-1-ol & 0.800 & 1.79 & 2.41 & 1.42 & 0.235 & 0.231 & 58.7 \\
\hline Propan-2-ol & 0.781 & 1.84 & 2.45 & 1.44 & 0.239 & 0.235 & 59.8 \\
\hline THF & 0.889 & 1.61 & 2.23 & 1.31 & 0.218 & 0.214 & 54.3 \\
\hline
\end{tabular}

\subsection{Electrolyte Additive}

\subsubsection{Calcium Chloride}

Where stated, $0.527 \mathrm{~mol} / \mathrm{dm}^{3}$ (5 wt\%) calcium chloride (anhydrous, Panreac, $95 \%$ ) was added to the water-based electrolyte. The effect of changing the weight percentage of the $\mathrm{CaCl}_{2}$ was tested, for example: $0,1,1.5,2,5,10,15,20$ or $25 \mathrm{wt} \%$ were added to the iodide/triiodide electrolyte and tested with and without RNCaSil in normal gasket cells. 


\subsubsection{Alternatives to Calcium}

Different sources of the chloride were tested, to ensure that it was the calcium that was affecting cell performance and not the chloride. $\mathrm{SrCl}_{2} \cdot 6 \mathrm{H}_{2} \mathrm{O}$ (Sigma Aldrich, $\geq 99 \%$ ) and $\mathrm{MgCl}_{2} \cdot 6 \mathrm{H}_{2} \mathrm{O}(\mathrm{Sigma}$ Aldrich, $\geq 99 \%)$ were used in amounts equimolar in the metal $\left(\mathrm{Sr}^{2+}\right.$ and $\left.\mathrm{Mg}^{2+}\right)$ to the $\mathrm{Ca}^{2+}$ used in the 5 wt $\%$ solution.

\subsection{3 $\mathrm{Ca}^{2+}$ Content Equivalent to that from RNCaSil}

Cells were tested with the electrolyte alone with $\mathrm{Ca}^{2+}$ added, the content of which was equivalent to the $\mathrm{Ca}^{2+}$ present in normal RNCaSil cells $\left(0.10 \mathrm{~g} \mathrm{RNCaSil}: 1.0 \mathrm{~cm}^{3}\right.$ electrolyte), assuming that all the calcium from RNCaSil was available. RNCaSil can be described by the formula $\mathrm{Ca}_{6} \mathrm{O}_{6} \mathrm{Si}_{15} \mathrm{O}_{30} \cdot 2 \mathrm{CaCO}_{3} \cdot \mathrm{Na}_{2} \mathrm{O} \cdot 2 \mathrm{H}_{2} \mathrm{O}$ (plus $30 \%$ adsorbed water). In $3.0 \mathrm{~g}$ of RNCaSil (the amount used in beaker cells, in which these tests were run), there was only $2.1 \mathrm{~g}$ of dry RNCaSil, which means there was $0.4389 \mathrm{~g} \mathrm{Ca}^{2+}$ present. $1.2158 \mathrm{~g}$ of $\mathrm{CaCl}_{2}$ delivered an equimolar amount of $\mathrm{Ca}^{2+}$.

\subsection{4 $\mathrm{Ca}^{2+}$ Only Added to Electrolyte}

An iodide/triiodide electrolyte was prepared where only $\mathrm{Ca}^{2+}$ was present and not $\mathrm{Cl}^{-}$. The same ratios of water, iodine and iodide normally used in the electrolyte were maintained, but with the iodide content from $\mathrm{CaI}_{2}$ rather than $\mathrm{KI}$. The electrolyte solution was normally prepared using DI water $(4.10 \mathrm{~g}), \mathrm{I}_{2}(0.10 \mathrm{~g})$ and $\mathrm{KI}(0.40 \mathrm{~g})$. Thus, there was $3.4096 \times 10^{-3} \mathrm{~mol}$ of iodide present and the amount of $\mathrm{CaI}_{2}$ required was $1.205 \times 10^{-3} \mathrm{~mol}$, or $0.354 \mathrm{~g}$. The $\mathrm{Ca}^{2+}$ content in this electrolyte was 55.7 mol\% of that in the $5 \mathrm{wt} \% \mathrm{CaCl}_{2}$ iodide/triiodide electrolyte.

\subsubsection{Tetrabutylammonium Hexafluorophosphate $\left(\mathrm{PF}_{6}^{+} \mathrm{N}\left(\mathrm{C}_{4} \mathrm{H}_{9}\right)_{4}\right)$}

Tetrabutylammonium hexafluorophosphate (Sigma Aldrich, $98 \%$ ) was added to the iodide/triiodide electrolyte using the following ratio: $\mathrm{I}_{2}(0.10 \mathrm{~g}), \mathrm{KI}(0.20 \mathrm{~g})$, tetrabutylammonium hexafluorophosphate $(0.010 \mathrm{~g})$, water (2 drops). This was then mixed with RNCaSil $(0.020 \mathrm{~g})$, and tested with C-coated ITOglass electrodes, in normal gaskets. 


\section{Synthesis of Tetraethoxyorthosilicate-Derived Gels}

The methods divulged in the literature for the condensation of tetraethoxyorthosilicate (TEOS, also called tetraethoxysiloxane $)^{[118-122]}$ were adapted, as they tended to result in non-gel products due to the metal sources added. The gels were formed from the condensation of TEOS $\left(2.0 \mathrm{~cm}^{3}, 0.00896 \mathrm{~mol}\right.$,

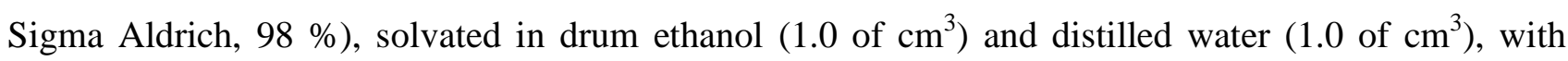
concentrated $\mathrm{HCl}\left(1.0\right.$ of $\mathrm{cm}^{3}, 0.01097 \mathrm{~mol}$, Pure Science Ltd, $\left.33.33 \%\right)$. This mixture was left stirring for 40 minutes. Drum ethanol $\left(0.5 \mathrm{~cm}^{3}\right)$ and distilled water $\left(0.5 \mathrm{~cm}^{3}\right)$ were then added to the mixture, prior to the metal additive $(0.00896 \mathrm{~mol}$ in the metal cation). The resultant mixture was then stirred for 2 hours, after which it was left overnight to set into a gel. The metal sources used were: $\mathrm{CaNO}_{3} \cdot 4 \mathrm{H}_{2} \mathrm{O}$ (Riedel-de Haën, $98 \%$ ), $\mathrm{SrCl}_{2} \cdot 6 \mathrm{H}_{2} \mathrm{O}$ (Sigma Aldrich, $99 \%$ ), $\mathrm{FeCl}_{3} \cdot 6 \mathrm{H}_{2} \mathrm{O}$ (Unilab, $96 \%$ ), $\mathrm{FeCl}_{2} \cdot 4 \mathrm{H}_{2} \mathrm{O}$ (Sigma Aldrich, $99 \%$ ), $\mathrm{CoCl}_{2} \cdot 6 \mathrm{H}_{2} \mathrm{O}$ (BDH Chemicals, $97 \%$ ), $\mathrm{MnSO}_{4} \cdot 4 \mathrm{H}_{2} \mathrm{O}(\mathrm{BDH}$ Chemicals, $>98$ $\%$ ), $\mathrm{CuNO}_{3} \cdot 3 \mathrm{H}_{2} \mathrm{O}$ (Riedel-de Haën, $99 \%$ ) and $\mathrm{ZnCl}$ (BDH Chemicals, > $98 \%$ ).

Some samples had slight variations to their solvent make-up, with respect to the ethanol content. For example: isopropyl alcohol (iPrOH) was used instead of ethanol; or a 50:50 ratio of ethanol and ethylene glycol was used instead of ethanol. In all samples, $50 \mathrm{wt} \%$ of the solvent was water to ensure solvation of the metal salts.

\section{$4 \quad$ Sodium Silicate and Ethylene Glycol Gels}

These were made from sodium silicate $\left(2.0 \mathrm{~cm}^{3}\right)$, water $\left(2.0 \mathrm{~cm}^{3}\right)$ and ethylene glycol $\left(3.0 \mathrm{~cm}^{3}\right)$, stirred for 2 minutes. $\mathrm{HCl}\left(0.5 \mathrm{~cm}^{3}, 1 \mathrm{~mol} / \mathrm{dm}^{3}\right)$ was then added, before stirring for a further 2 minutes and adding ethylene glycol $\left(3.0 \mathrm{~cm}^{3}\right)$. The metal solution $\left(\mathrm{CaI}_{2}(8.55 \mathrm{mmol})\right.$, the metal cation $(0.95 \mathrm{mmol})$ and water $\left.\left(1.0 \mathrm{~cm}^{3}\right)\right)$ was stirred rapidly into the mixture and the gel allowed to develop overnight. The source materials for the metals used were those listed above (Part III 3). A blank gel (or control) was tested, which only consisted of the sodium silicate, water and ethylene glycol, and no metal solution.

\section{$5 \quad$ Gasket Cell Fabrication}

Some tests were carried out between two small pieces of indium tin oxide coated glass (ITO glass, Sigma Aldrich, 70-100 $\Omega / \mathrm{sq}, 0.107 \mathrm{~cm}$ thickness), as shown in Figure 13. The surface area and volume 
of samples was controlled by a Teflon gasket of known dimensions. The Teflon gasket was held in place with petroleum wax. Contacts were soldered on with indium.

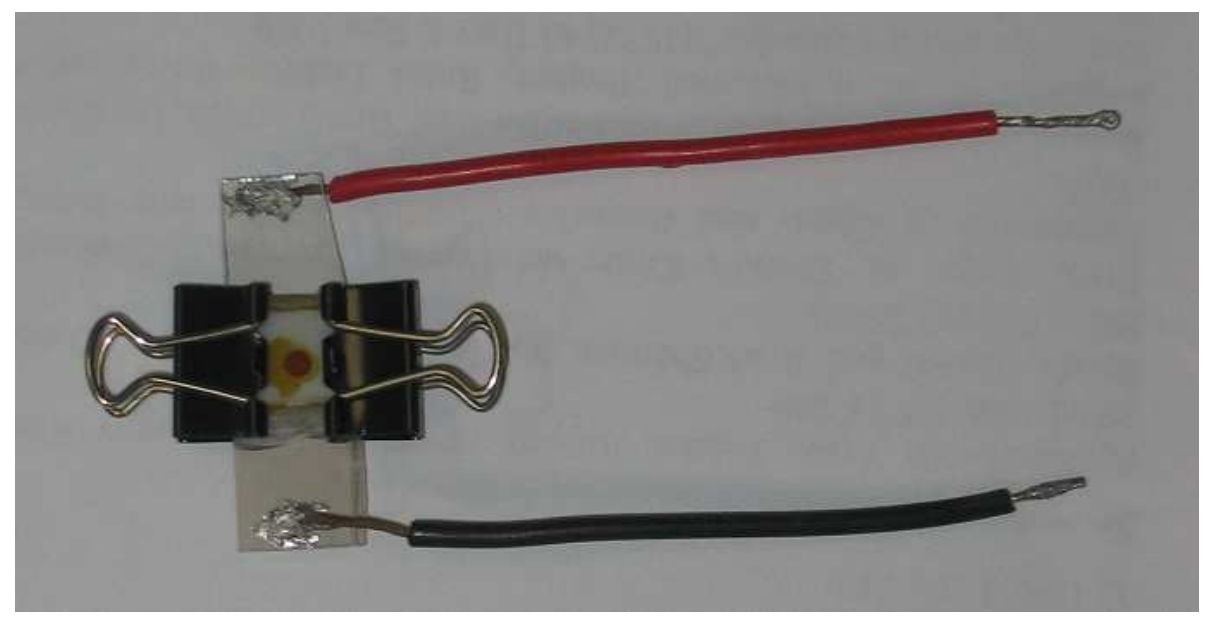

Figure 13. "Gasket Cell"

\subsection{Electrode Coating}

The back electrode (usually either ITO glass or ITO-coated PET) was carbon, platinum or gold coated. For most of the NCaSil cells, the back electrode was carbon coated using a JEOL, JEC 560, Auto C Coater, to give a coating of approximately $7 \mathrm{~nm}$ thickness. With the TEOS cells, the back electrode was platinum coated in a JEOL JFC-1500 Ion Sputtering Device, also at $7 \mathrm{~nm}$ thickness. Platinum coating was used instead of carbon coating where the sample's acidity or longer-term testing caused any carbon coating to be stripped off the electrode. Any gold coating was done with a Fisons Instruments Polaron SC500 Sputter Coater, set to $7 \mathrm{~nm}$. Gold coating was only used for comparative studies of different coatings. In some experiments, the back electrode was constructed using carbon tape on "normal" (nonITO-coated borosilicate) glass.

\section{2 "Normal" Gasket Cells}

Unless otherwise stated, the NCaSil was not sintered onto the photoelectrode, nor was any additional light-absorbing dye used. For most investigations, RNCaSil was chosen due to its higher stability compared to NCaSil. Values reported herein are predominantly averages of two or, in most cases, three repetitions. "Normal" gasket cells were fabricated with a $5.05 \mathrm{~mm}$ diameter and distance of $0.052 \mathrm{~cm}$ between the electrodes (i.e., sample areas of $0.200 \mathrm{~cm}^{2}$ and volumes of $0.0104 \mathrm{~cm}^{3}$ ). A ratio of RNCaSil:electrolyte solution of $0.10 \mathrm{~g}: 1.0 \mathrm{~cm}^{3}$ was used, unless otherwise indicated. 


\subsection{Cells From Slurry-Form NCaSil}

In most cases, the dry powder form of NCaSil was used and the ratio of NCaSil to electrolyte given as a weight:volume ratio. However, when a slurry or cake form was used, the sample was made up without any additional water and the solid content, $\mathrm{KI}$ and $\mathrm{I}_{2}$ ratio kept consistent with comparable experiments. For example: the most common powder NCaSil:electrolyte ratio used was $0.10 \mathrm{~g}$ NCaSil: $1.0 \mathrm{~cm}^{3}$ electrolyte. The normal electrolyte solution, as stated above, was prepared from $0.10 \mathrm{~g}$ $\mathrm{I}_{2}, 0.40 \mathrm{~g} \mathrm{KI}, 4.10 \mathrm{~g}$ DI water, $0.24 \mathrm{~g} \mathrm{CaCl}_{2}$, making a total weight of $4.84 \mathrm{~g} .1 .0 \mathrm{~cm}^{3}$ of this solution weighed $1.1325 \mathrm{~g}$.

Table 6. Formulation of the Normal Iodide/Triiodide Electrolyte Used.

\begin{tabular}{lccc}
\hline Electrolyte Solution & Mass $(\mathrm{g})$ & Mass $(w t \%)$ & In $1.0 \mathrm{~cm}^{3}(\mathrm{~g})$ \\
\hline $\mathrm{I}_{2}$ & 0.10 & 2.07 & 0.0234 \\
$\mathrm{KI}$ & 0.40 & 8.26 & 0.0935 \\
$\mathrm{DI}$ Water & 4.10 & 84.71 & 0.9593 \\
$\mathrm{CaCl}_{2}$ & 0.24 & 4.96 & 0.05617 \\
\hline
\end{tabular}

If a slurry was, for example, $7 \mathrm{wt} \%$ solids, then $1.4286 \mathrm{~g}$ of the slurry was required for $0.10 \mathrm{~g}$ of solid. For a $\mathrm{Ca}^{2+}$ free sample, $0.0234 \mathrm{~g}$ of $\mathrm{I}_{2}$ and $0.0935 \mathrm{~g}$ of KI was added to the NCaSil slurry. For a sample with added $\mathrm{Ca}^{2+}, 0.05617 \mathrm{~g} \mathrm{CaCl}_{2}$ was also mixed into the samples.

\subsection{TEOS-Gel Gasket Cells}

When TEOS-gel samples were tested with the $70 \%$ ethanol iodide/triiodide electrolyte, $0.40 \mu \mathrm{g}$ or 0.95 $\mu \mathrm{g}$ of the electrolyte was added to $0.0104 \mathrm{~cm}^{3}$ of the TEOS-gel sample. The samples were tested in normal gasket cells, with Pt-coated dark electrodes, as the acidity of the samples would have removed carbon coating easily.

\section{5 $\quad \mathrm{SiO}_{2}$ and $\mathrm{TiO}_{2}$ Testing}

$\mathrm{SiO}_{2}$ (precipitated, BDH Lab Reagent) and $\mathrm{TiO}_{2}$ (anatase, BDH Lab Reagents, $98 \%$ ) testing was conducted in the same fashion as NCaSil in normal gasket cells. ITO-glass electrodes were used, with 
the dark electrodes carbon coated. Cells were made up with $0.10 \mathrm{~g}$ of oxide and $1.0 \mathrm{~cm}^{3}$ of either 0 or 5 wt $\% \mathrm{CaCl}_{2}$ water-based iodide/triiodide electrolyte.

\section{Beaker Cells}

Other samples were tested in beakers, as shown in Figure 14. These cells were made up in Simax (borosilicate glass) beakers. Electrodes were used at distances of approximately 0.5, 2.0 and $2.5 \mathrm{~cm}$. The electrodes were kept in place by a plastic holder, with slits separated by the given distances. Unless otherwise stated, the beaker cells predominantly used an electrode distance of $2.0 \mathrm{~cm}$. All cells contained $3.0 \mathrm{~g}$ of $\mathrm{NCaSil}$ and $30 \mathrm{~cm}^{3}$ of electrolyte (the same ratio as in normal gasket cells).

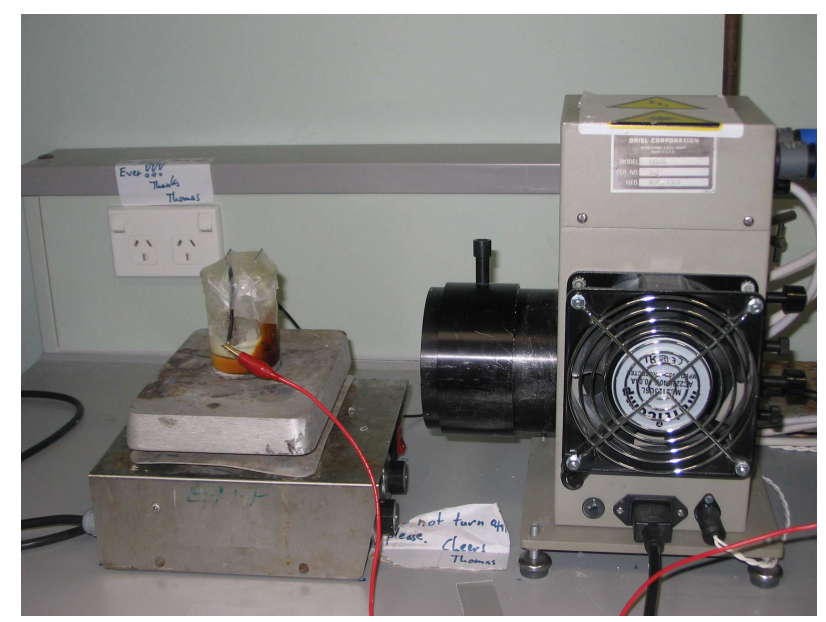

Figure 14. “Beaker Cell” and Research Arc Lamp

ITO glass is indium tin oxide coated onto normal glass (not quartz glass). Because of this, the samples in cells were cut off from all incoming light of wavelengths below approximately $295 \mathrm{~nm}$. Preliminary tests performed showed that, as a consequence of this, using a quartz beaker did not improve cell performance over using a normal glass beaker (SIMAX, borosilicate glass).

\subsection{Electrode Position}

The RNCaSil beaker cell was examined with the electrodes in three different positions relative to the light; with the electrodes either parallel to the light beam (Figure 15(a)), or perpendicular to the lamp with the C-coated ITO electrode towards the back of the cell (as a normal dark electrode, Figure 15(b)) or closest to the light (where the uncoated photoelectrode usually is, Figure 15(c)). This was done with three samples, with electrode distances of approximately $0.5,2.0$ and $2.5 \mathrm{~cm}$. 
(a)

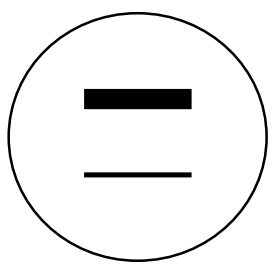

(b)

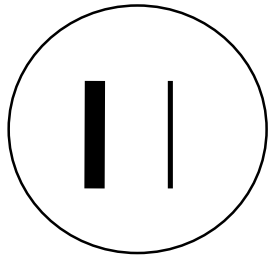

(c)

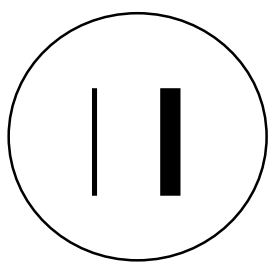

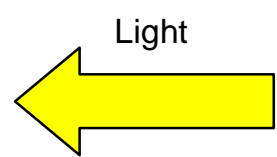
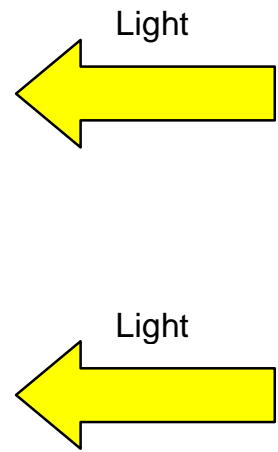

Figure 15. Different Electrode Positions Tested (Birds-Eye View of Beaker Cell): (a) Electrodes Parallel to the Light Source; Electrodes Perpendicular to the Light Source, (b) C-coated ITO to the Back, and (c) C-coated ITO to the Front.

\section{$7 \quad$ Quartz Cuvette Cells}

It was tested whether the back ITO-glass electrode was required (as is usually the case for Graetzel type DSSC), or whether a thin Pt-wire was sufficient (as in a photogalvanic cell). Tests done in quartz cuvettes used a ratio of RNCaSil:electrolyte of $1.0 \mathrm{~g}: 1.0 \mathrm{~cm}^{3}$, where the electrolyte used was $70 \mathrm{wt} \%$ ethanol $30 \mathrm{wt} \%$ DI water solvated iodide/triiodide. The cuvette had an (inside) depth of $0.994 \mathrm{~cm}$. ITOglass electrodes used were $2.50 \times 0.97 \mathrm{~cm}$. The Pt-wire used had a thickness of $0.30 \mathrm{~mm} .8 .0 \mathrm{~cm}$ of Ptwire was immersed in the samples.

Four different electrode variations were tested (Figures 16 and 17). First, two ITO-glass electrodes were used, the back electrode being platinum coated. This was a blank sample to compare to the Ptwire samples. Secondly, an ITO-glass photoelectrode and Pt-wire back electrode combination was tested. Thirdly, the Pt-wire was brought to the front of the cell and uncoated ITO glass used as the back electrode. And, finally, to determine the effect of the lower wavelengths of light let through by the quartz cuvette and the Pt-wire, a sheet of ITO glass was placed in front of cell-type three. 


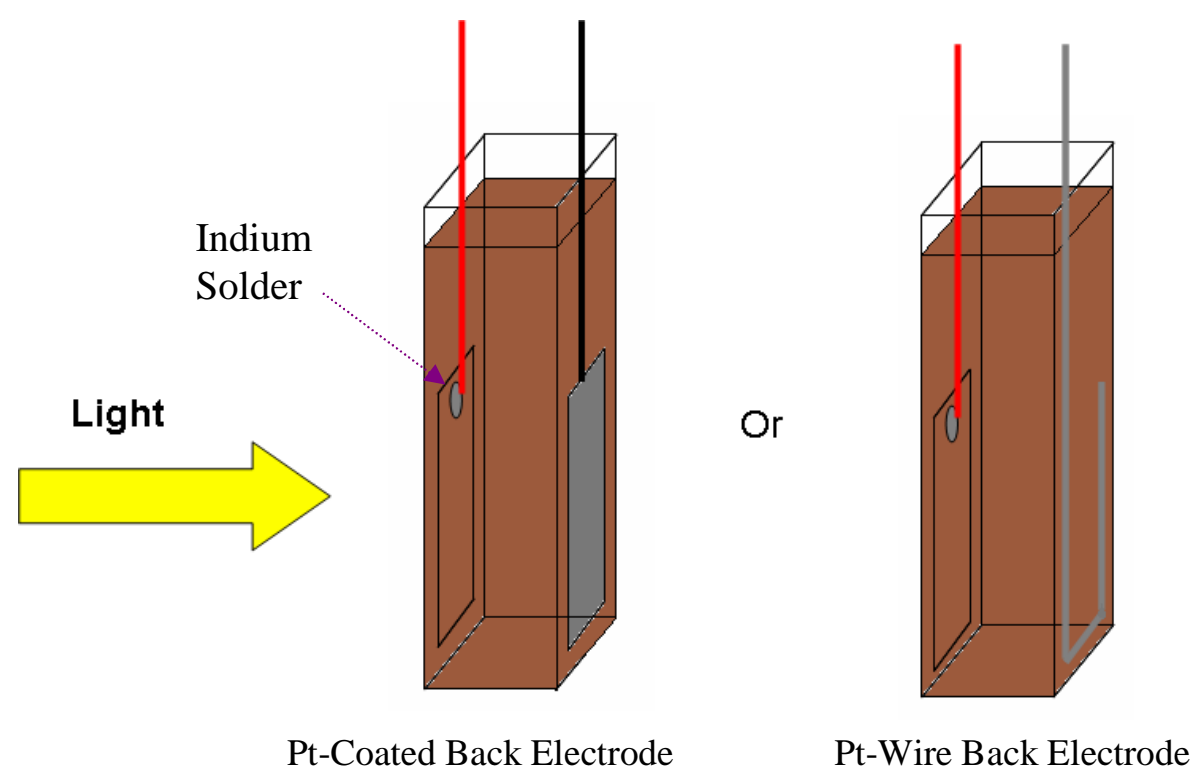

Figure 16. Quartz Cuvette Testing: ITO Glass Front Electrode and Either a Pt-Coated or Pt-Wire Back Electrode.

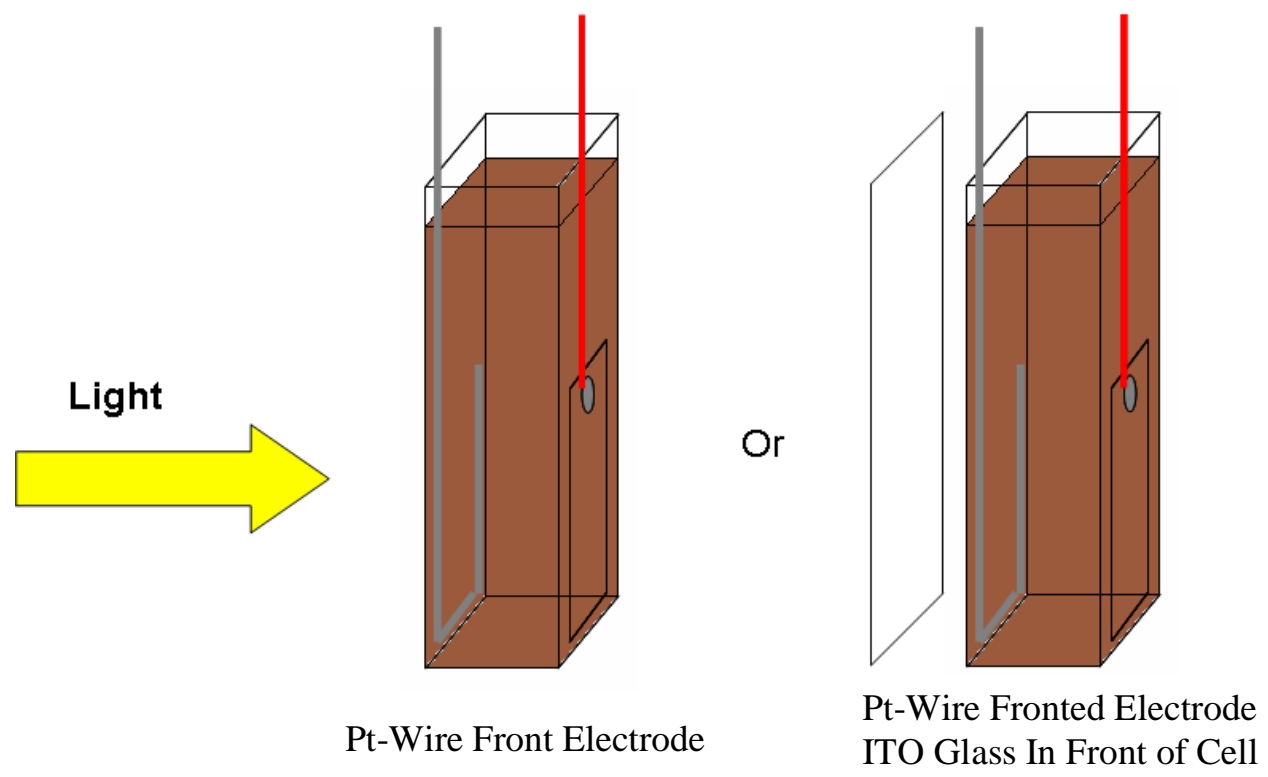

Figure 17. Quartz Cuvette Testing: ITO Glass Back Electrode and a Pt-Wire Front Electrode, with or without ITO Glass in Front of the Cell.

\section{$8 \quad$ Testing the Effect of Cell Width and Depth}

\subsection{Different Gasket Cells}

Different cell widths and depths were tested by varying the Teflon gasket used in the gasket cells to known dimensions. To test cell width, and thus the effect of surface area, the same Teflon used to make 
the "normal" gaskets was employed to make gaskets of different surface areas, but consistent depth. The diameters tested were: 2.53, 5.05 ("normal"), 7.50. 10.16 and $15.09 \mathrm{~mm}$.

In order to test the effect of cell depth, Telfon tape (Ceelon) of $0.08 \mathrm{~mm}$ thickness was used (compared to a normal gasket of $0.52 \mathrm{~mm}$ thickness). Due to a technical limitation, the sample surface area of the Teflon tape cells $\left(6.00 \mathrm{~mm}\right.$ diameter, $\left.1.13 \mathrm{~cm}^{2}\right)$ was not quite the same as that of the "normal" gaskets $\left(5.05 \mathrm{~mm}\right.$ diameter, $\left.0.80 \mathrm{~cm}^{2}\right)$.

\subsection{Slotted Vessel Testing}

To further investigate the effect of electrode distance on cell performance, a slotted vessel was constructed from Teflon and quartz glass. This water-tight vessel was cut with slits able to hold ITOcoated glass slides, allowing for the measurement of cell performance of cells with different electrode distances (Figure 18).

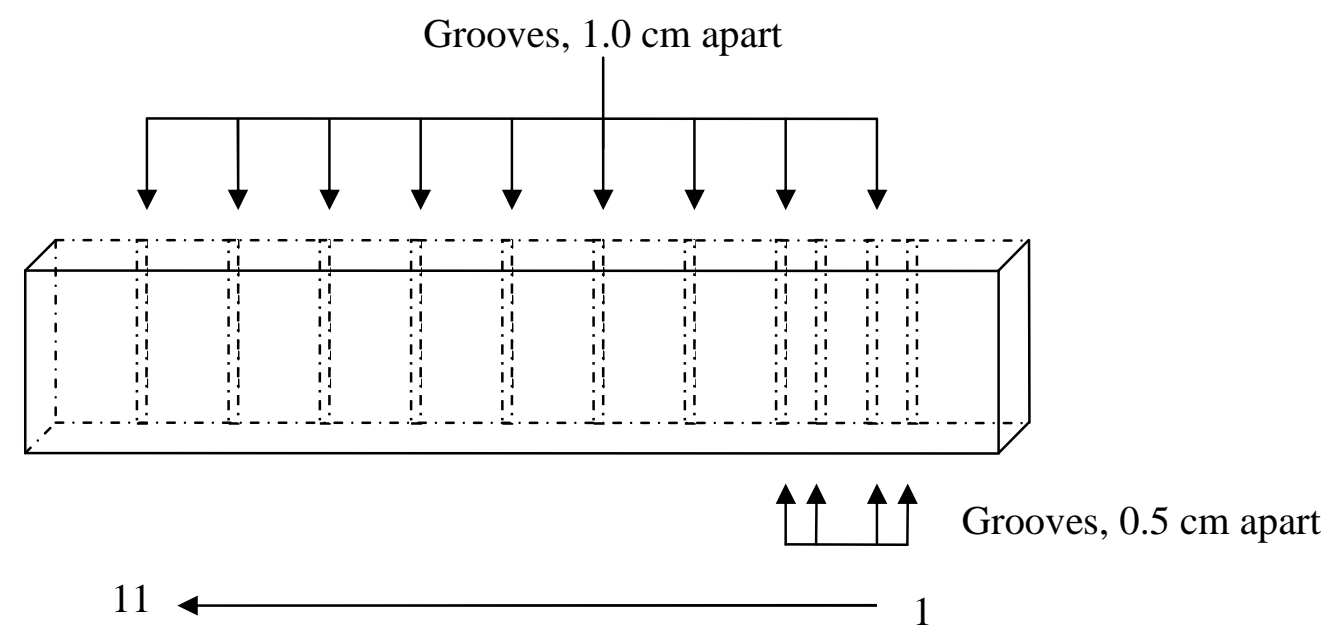

Figure 18. Side Panel of the Slotted Vessel used to Test the Effect of Electrode Distance.

The front panel (through which the light entered the vessel) was constructed from quartz glass. Given that the first slit had to hold the photoelectrode, the distances tested were $0.5,1.0,1.5,2.5,3.5,4.5,5.5$, 6.5, 7.5 and $8.5 \mathrm{~cm}$ (Figures 19 and 20). Tests were either performed with the "gap" between the quartz glass and the first slot (the photoelectrode) filled or unfilled. 


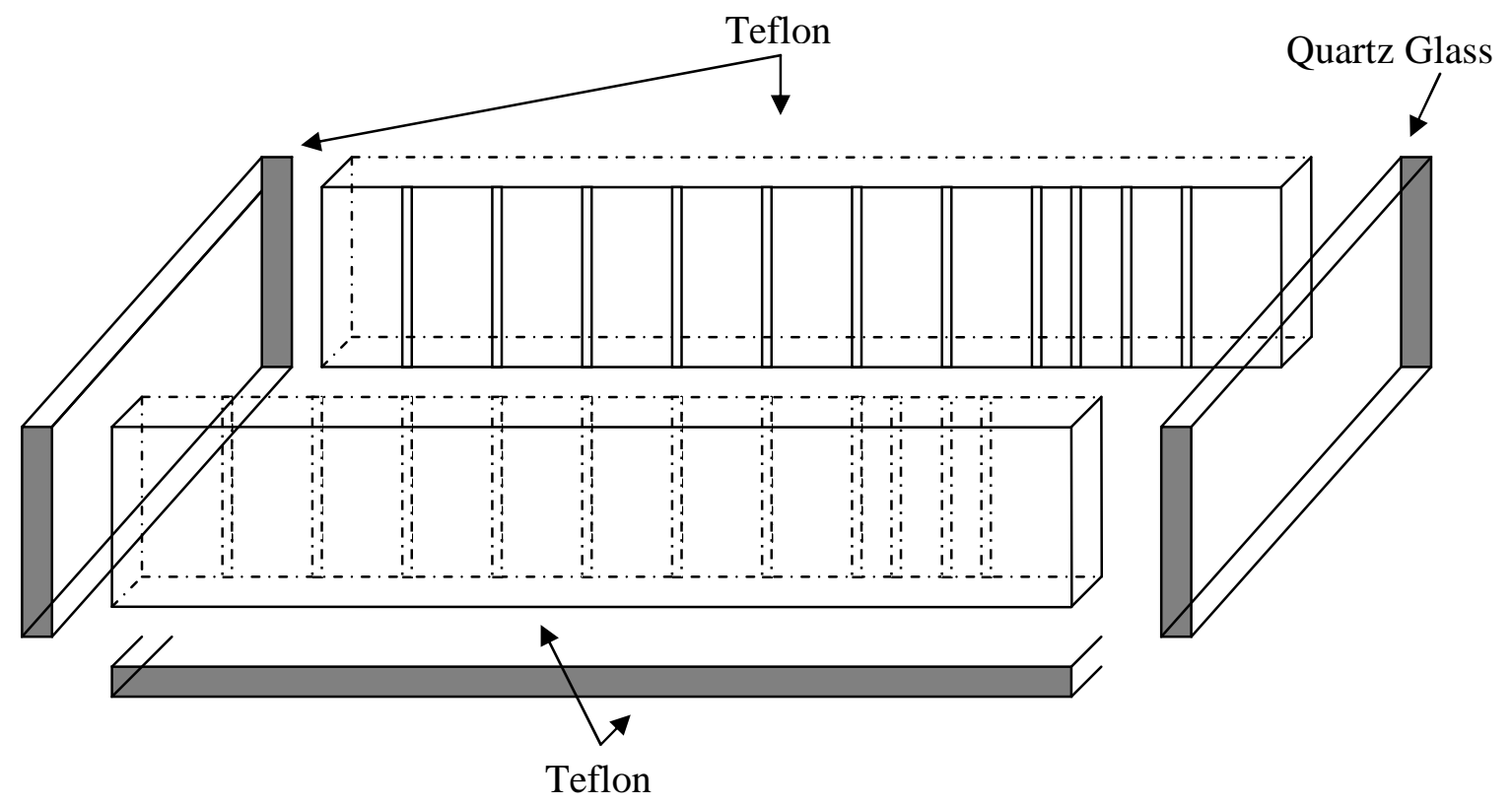

Figure 19. Slotted Vessel used to Test the Effect of Electrode Distance.

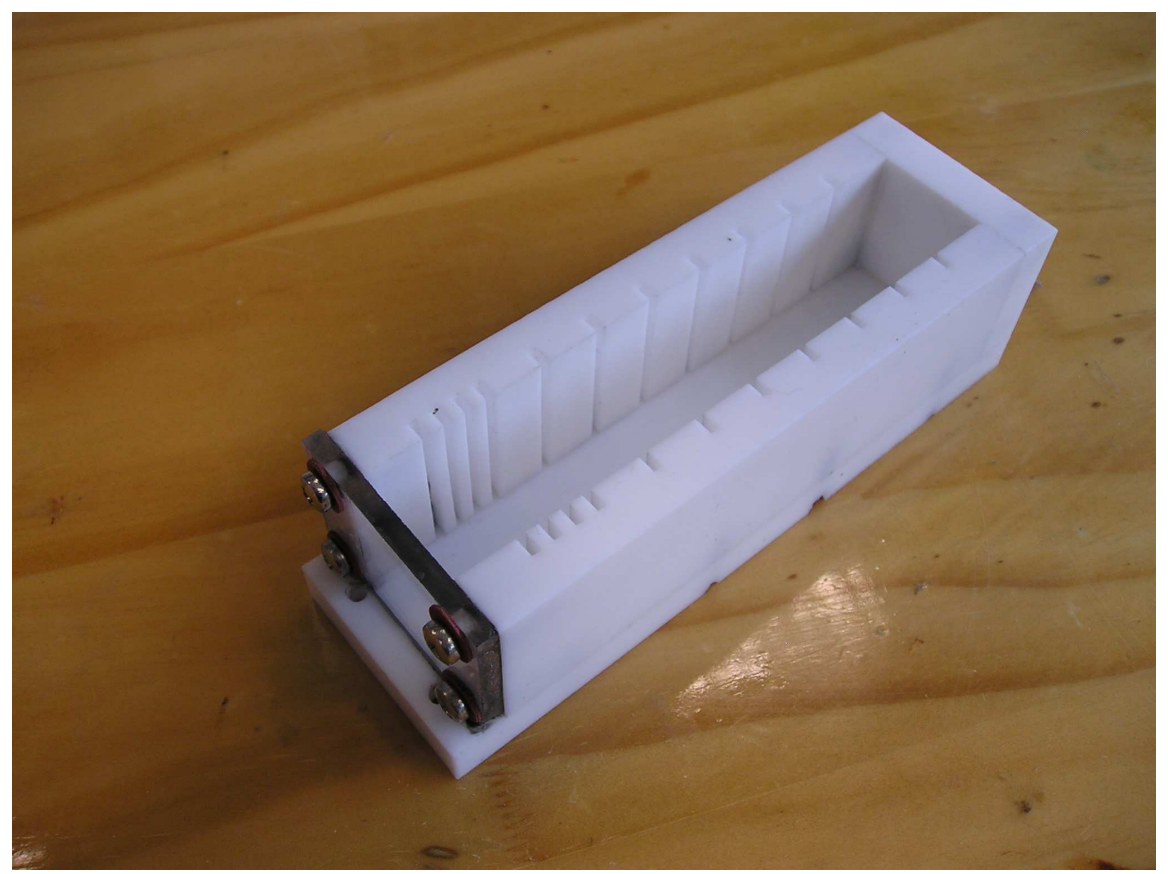

Figure 20. Slotted Vessel.

\section{$9 \quad$ Stirring and Non-Stirring}

Some beaker tests (Simax, borosilicate glass) were carried out using an overhead stirrer (Figure 21). This experiment employed RNCaSil in a water-based iodide/triiodide electrolyte $\left(3.0 \mathrm{~g}: 30 \mathrm{~cm}^{3}\right)$. ITOglass electrodes were used at distances of approximately $2.0 \mathrm{~cm}$, kept in place by a plastic holder. The back electrode was C-coated. 


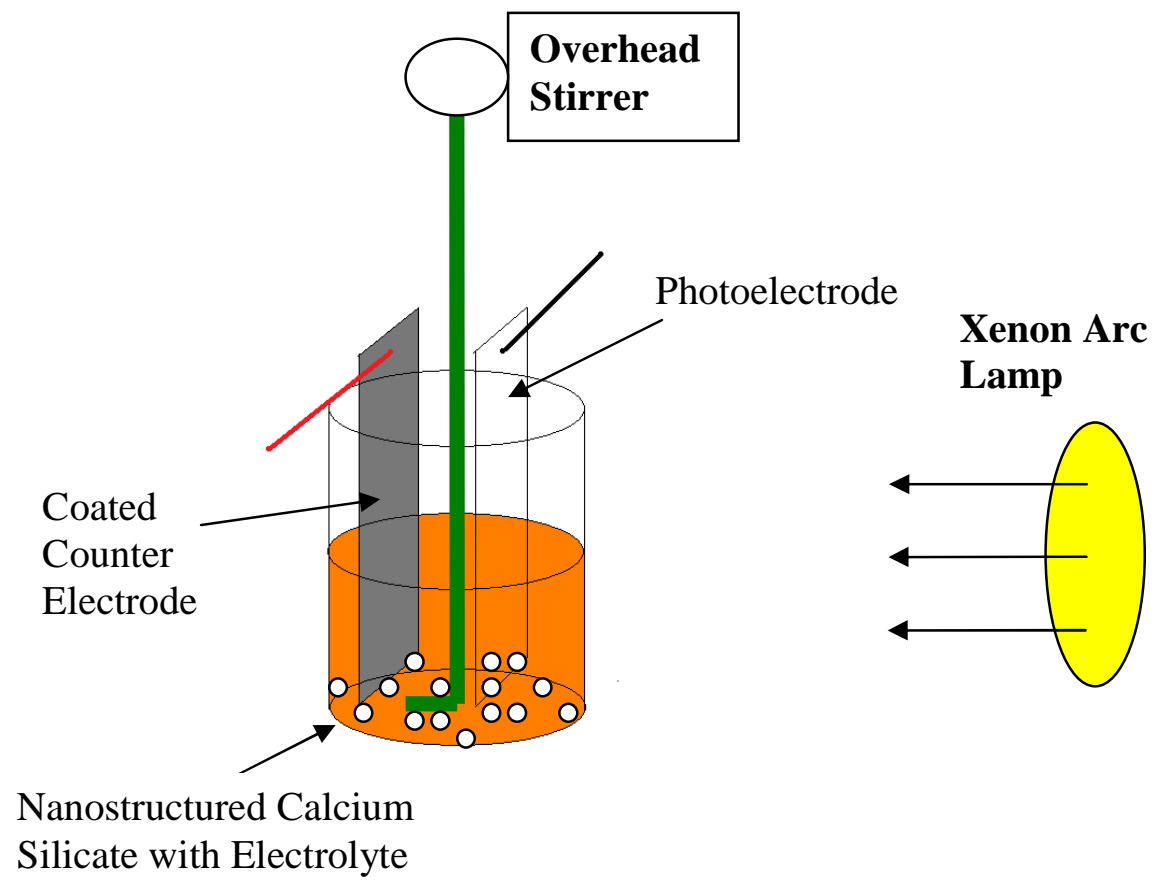

Figure 21. Overhead Stirring of a Beaker Cell.

\section{$10 \quad$ Plastic Electrodes}

\subsection{Normal Plastic Electrode Cells}

Cells were fabricated with ITO-coated polyethylene terephthalate (ITO PET, 35, 60 and $100 \Omega$, Sigma Aldrich) electrodes rather than ITO glass. These cells were fabricated in the same fashion as normal gasket cells (normal Teflon gasket, Pt-coated dark electrode, uncoated front electrode), except that the ITO PET was used rather than ITO glass.

\subsection{Laminated Plastic Electrode Cells}

The construction of these cells was almost identical to the normal ITO-PET cells, as outlined above, with the following exceptions:

- The Teflon gasket was glued to the Pt-coated ITO PET (100 $\Omega$ ), rather than held in place with petroleum wax, to avoid wax melting into the sample during the lamination process. 
- The normal gasket cells with ITO glass and normal ITO-PET cells utilised plastic coated $\mathrm{Cu}$ wire connections. However, due to size constraints of the laminator, the laminated cells used non-plastic coated $\mathrm{Sn}$ - or $\mathrm{Cu}$-wire connections.

- All dark electrodes were Pt-coated.

The entire cell, including the connections in their entirety, was laminated at $72{ }^{\circ} \mathrm{C}$ (Figure 22). The ends of the connecting wires were subsequently freed to make measurements.

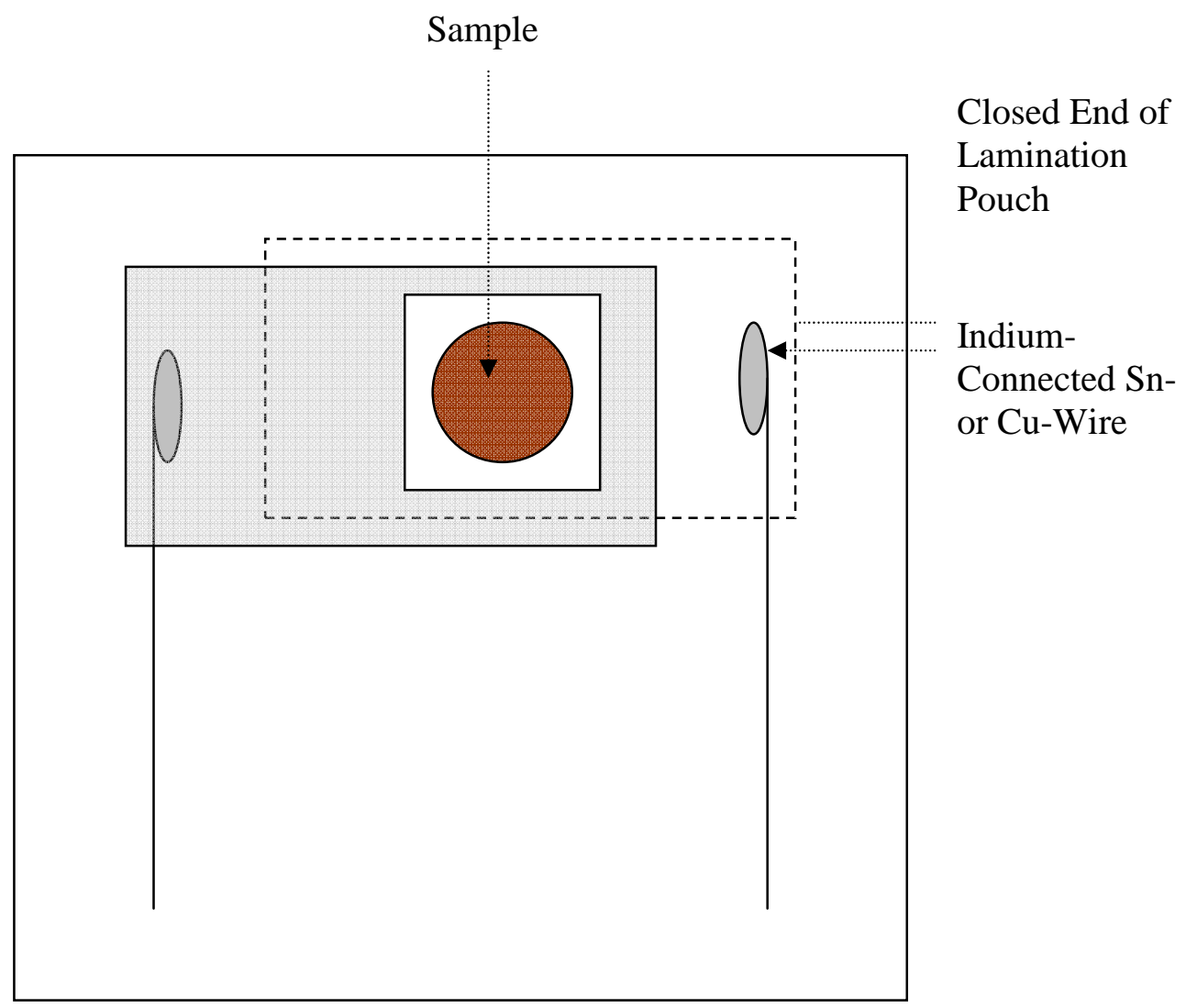

Figure 22. Representation of Laminated ITO-PET Cells.

\section{Sintering NCaSils}

\subsection{Silver Paint}

Silver paint was coated onto ITO glass, before RNCaSil was pressed into the paint. This was then allowed to dry, before being tested with a water iodide/triiodide electrolyte. 


\subsection{Using Sodium Silicate as a Binder}

Sodium silicate $\left(6.5 \mathrm{~g}\right.$, Orica, $\left.28.5 \% \mathrm{SiO}_{2}\right)$ was mixed with RNCaSil $(0.60 \mathrm{~g})$. ITO glass was coated with this mixture by leaving the glass in plastic Petri dishes $(5.3 \mathrm{~cm}$ diameter $)$, immersed in the mixture. Surfaces of the glass required for contacts were masked with Teflon tape. This was left for 48 hours, before testing when used in conjunction with a $70 \mathrm{wt} \%$ ethanol iodide/triiodide electrolyte solution (Pt-coated ITO-glass dark electrode).

\subsection{Hydrothermal Treatment}

A slurry of NCaSil (20 g) was prepared, such that overall the slurry consisted of $5 \mathrm{wt} \% \mathrm{NCaSil}$. Sodium silicate (Orica, $28 \mathrm{wt} \% \mathrm{SiO}_{2}$ ) was added to this, such that the amount was $10 \mathrm{wt} \%$ of the previous mixture (i.e., $2 \mathrm{~g}$ ). Teflon tape was used to safeguard any areas of the ITO glass required for connections. The glass was placed into the bomb $\left(200 \mathrm{~cm}^{3}\right)$, the ITO-coated side facing upwards, and the slurry was poured into the bomb over the glass. This was then autoclaved for 3 hours at $210^{\circ} \mathrm{C}$ in a McGregor kiln, before being allowed to cool overnight. This process was repeated for the same sheet of glass, resulting in a double-coated slide and a coating of NCaSil of approximately $15 \mu \mathrm{m}$.

\section{Dye Sensitiser}

As the ruthenium dye sensitiser is the most expensive component of the cell, a sensitiser was not used for every cell. Figure 23 shows the ruthenium complex used as a dye, tris(2,2'-bipyridyl)dichlororuthenium (II) hexahydrate (Sigma Aldrich, $99.95 \%$ ).

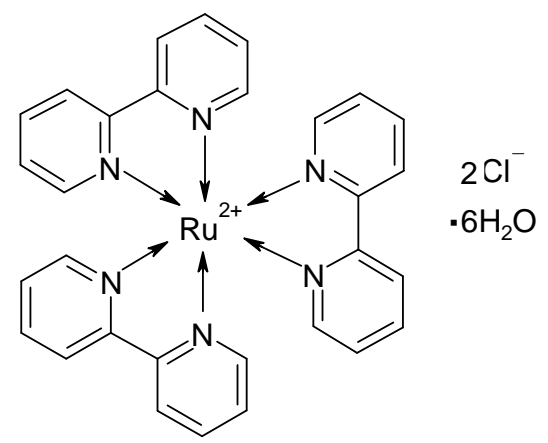

Figure 23. Tris(2,2'-bipyridyl)dichloro-ruthenium (II) hexahydrate (Sigma Aldrich). 
Using reported methods of dye-sensitising $\mathrm{TiO}_{2}$ Graetzel cells as a guideline,${ }^{[23,40]} \mathrm{RNCaSil}(0.10 \mathrm{~g})$ was sensitised by immersing it in an absolute ethanol solution of the dye $\left(10 \mathrm{~cm}^{3}, 250 \times 10^{-6} \mathrm{~mol} / \mathrm{dm}^{3}\right)$. This mixture was left in a Labnet Orbit 300 shaker, at 300 r.p.m for 20 hours, before filtering by vacuum and washing with drum ethanol. The remaining yellow-orange solid was placed in an oven at $105{ }^{\circ} \mathrm{C}$ for 72 hours.

The resulting dyed RNCaSil was tested with a $70 \mathrm{wt} \%$ ethanol $30 \mathrm{wt} \%$ water iodide/triiodide electrolyte $\left(0.10 \mathrm{~g}: 1.0 \mathrm{~cm}^{3}\right)$, in a normal gasket, ITO-glass cell, with a Pt-coated back electrode.

\section{Light Source Used}

The incident light from the Newport Oriel, ozone-free xenon-arc discharge lamp used had an electrical power output of $75 \mathrm{~W}$ (see Figure 37 below for the irradiance spectrum at $0.50 \mathrm{~m}$ ). ${ }^{[123]}$ The relevant area of the irradiance spectrum $(200-700 \mathrm{~nm}$, see Figure 27, which showed that no light uptake occurs above $700 \mathrm{~nm}$ ) was approximately $3.85 \mathrm{~W} / \mathrm{m}^{2}$. Samples were tested at $0.30 \mathrm{~m}$ and, as irradiance varies roughly in proportion to the distance squared, the irradiance was increased by a factor of $(0.50 / 0.30)^{2}=$ 2.79 , giving $10.74 \mathrm{~W} / \mathrm{m}^{2}$. This light was focused by 2.0 fold, resulting in an optical power of 21.47 $\mathrm{W} / \mathrm{m}^{2}$. No adjustments were made for energy loss through the lens, glass or size effects.

\section{Cell Performance and Analysis}

Cells were tested in a black box, free from external light. Cell performance was evaluated with a variable resistor to measure voltage and current on change in resistance (Figure 24).

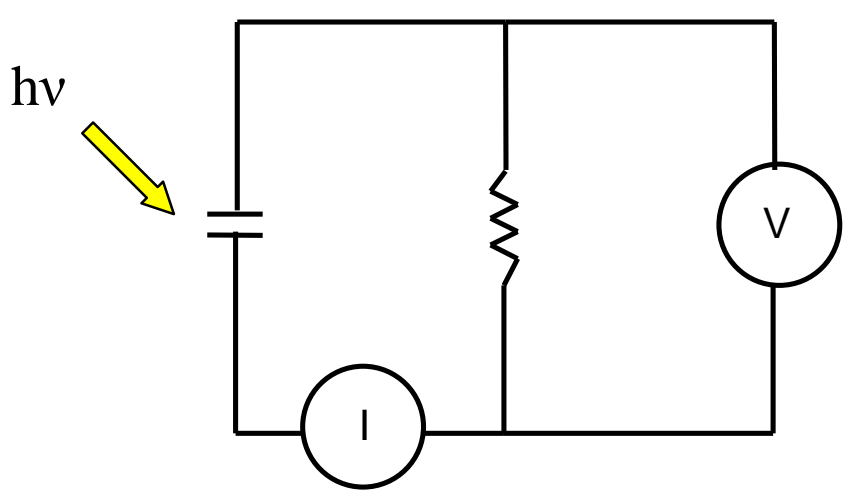

Figure 24. Circuit Diagram of Set Up Used to Measure Cell Performance. 
Current and voltage were plotted to measure the total power output and overall efficiency. Overall cell power conversion efficiencies were determined using equation 6 (see above), where the light intensity of the incident light was defined as $21.47 \mathrm{~W} / \mathrm{cm}^{2}$.

\section{Optical Properties}

Optical properties were measured with a HunterLab ColorQuest on the CIE scale and values were averaged over a minimum of 5 samples.

\section{Optical Density Filters}

Optical density filters (Edmund Industrial Optics) were employed. These were based on a logarithmic scale, such that optical density $(\mathrm{OD})=\log _{10}\left(\mathrm{I}_{\mathrm{o}} / \mathrm{I}\right)$, where $\mathrm{I}_{\mathrm{o}}$ is the initial intensity and $\mathrm{I}$ the resultant intensity. In tests where these filters were used, the voltage and current for cells with five different filters, which were sequentially placed in the light path, were compared to an unfiltered cell. The optical density of the filters and the corresponding light intensities are listed in Table 7.

Table 7. Optical Density Filters and their Corresponding Light Intensities.

\begin{tabular}{cc}
\hline OD Filter & Light Intensity (I) \\
\hline 0.00 & 1.0000 \\
0.15 & 0.7079 \\
0.30 & 0.5012 \\
0.40 & 0.3981 \\
0.90 & 0.1259 \\
2.50 & 0.0032 \\
\hline
\end{tabular}

\section{Surface Area Measurements}

Dry NCaSil surface areas were measured by nitrogen absorption/desorption using the Brunauer, Emmett and Teller (BET) Isotherm, with a Micrometrics Gemini V Surface Area and Pore Size Analyzer. 


\section{Particle Size Analysis}

Particle size analysis was carried out an a Malvern Instruments Mastersizer 2000 for spherical particles in water, at a particle refractive index of 1.610, dispersant refractive index of 1.330 , absorption of 0.1 , over the size range of 0.020 to $2000 \mu \mathrm{m}$ and averaging over 20000 samples.

\section{Viscosity Measurements}

The viscosities of the different electrolyte solutions were measured with a KSV Vibro Viscometer SV10 , using a blade method. 


\section{RESULTS AND DISCUSSION}

\section{Different Forms of Nanostructured Calcium Silicate}

In this chapter, unreinforced, reinforced and metal-doped nanostructured calcium silicates (NCaSils) tested with the iodide/triiodide electrolyte (with and without $5 \mathrm{wt} \% \mathrm{CaCl}_{2}$ ) in gasket cells (Experimental, Part III 5) are investigated. Cell efficiencies are compared, the absorbance range of NCaSil-containing cells assessed by UV-vis and the relationship between the electrolyte and NCaSil studied by UV-vis and cyclic voltammetry. The chapter concludes with a short discussion on the importance of mixing order when forming the NCaSils and issues with consistency of CM-RNCaSil.

\subsection{Unreinforced and Reinforced NCaSils}

Untreated, unreinforced NCaSil proved to have the lowest cell performance of all the different NCaSils tested, when the electrolyte was free from any $\mathrm{Ca}^{2+}$ additive (Figure 25). This was not due to its exhibited $V_{o c}$, which, as is discussed in the following paragraphs, did not vary greatly between the different NCaSils. The poor performance was the result of a low $I_{\mathrm{sc}}$, which was attributed to its lower surface area and the instability of its microstructure in the presence of the solvent. The $I_{\mathrm{sc}}$ was significantly improved upon the addition of $5 \mathrm{wt} \% \mathrm{CaCl}_{2}$ to the electrolyte solution. This increase was attributed to the enhanced presence of the mobile $\mathrm{Ca}^{2+}$ ion and was observed with all NCaSils tested.

NCaSil comprises of a silicate backbone with calcium and silanol groups on the surface. When dried, the silanol groups experience a strong attractive force to each other and to surface calcium. Combined with forces effected by the high surface tension of water, the silicate structure can collapse, as plates are pulled towards each other. NCaSil can be modified or reinforced to prevent loss of structure and surface area upon drying, and increase stability and cell performance. Consequently, reinforced (RNCaSil), acid washed (AWNCaSil) and ethoxyethanol-treated NCaSil (EENCaSil) were tested and compared to unreinforced NCaSil (dry and wet, filter cake-form).

$V_{o c}$ was not found to vary greatly between the different forms of NCaSils. Most variances observed fell within the uncertainties of the experiment. $V_{o c}$ increased slightly on addition of $5 \mathrm{wt} \% \mathrm{CaCl}_{2}$ to the electrolyte (Figure 25(a)). This could be attributed to the addition of the $\mathrm{Ca}^{2+}$ changing the inherent 
structure of the NCaSil and, hence, its band gap. However, as there was little evidence that NCaSil was acting as a semiconducting material (discussed further in the following section), it was more likely a surface effect. The interaction of $\mathrm{Ca}^{2+}$ with the NCaSil surface affected the iodide/triiodide-surface interaction, as will be discussed in greater depth below (Part IV 4.3.1, 9.6 and 12), increasing the $V_{o c}$. This affect on $V_{o c}$ was supported by cyclic voltammetry, discussed below (Part IV 1.1.5).
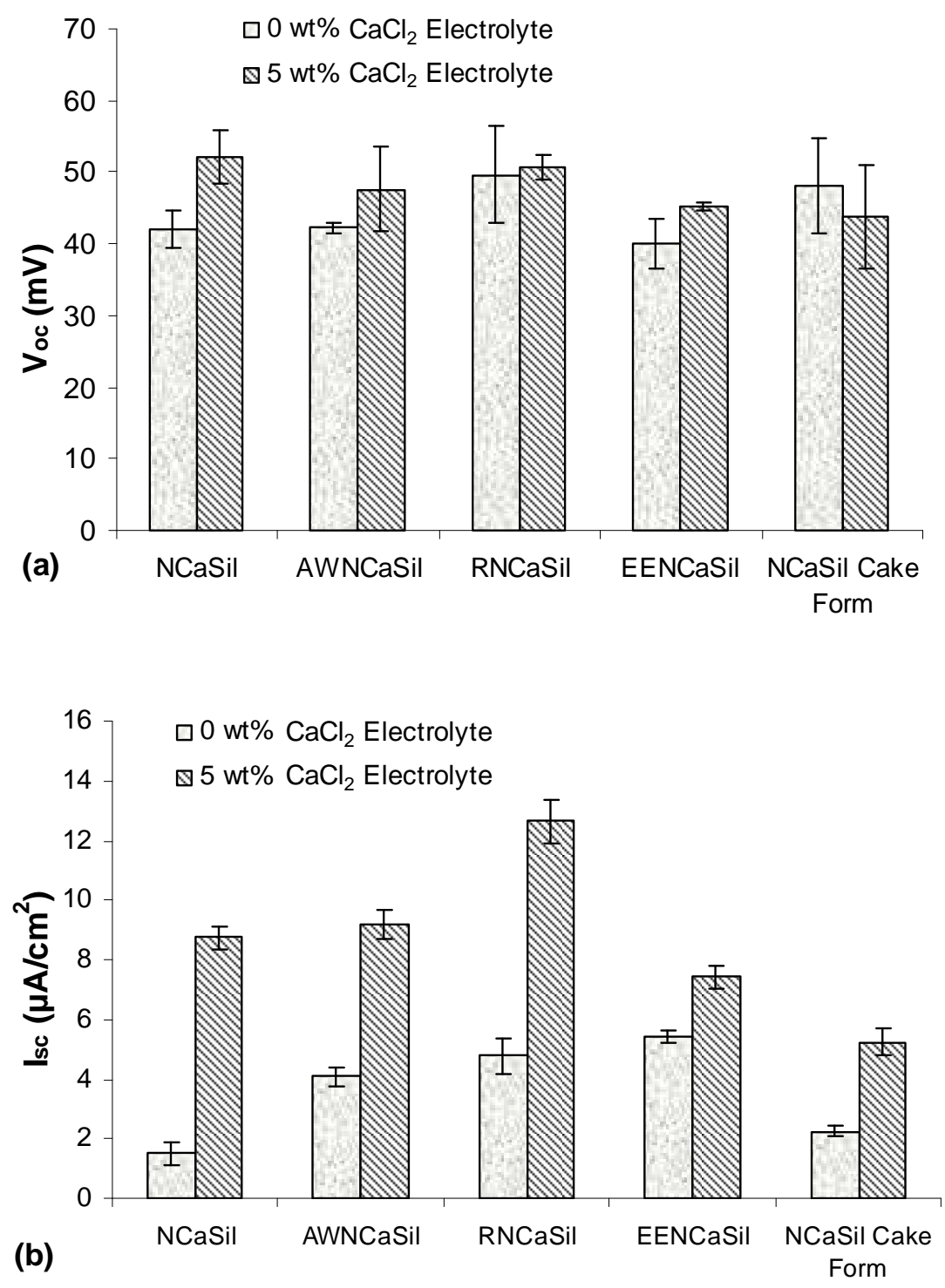

Figure 25. Different $\mathrm{NCaSils}$ with 0 or $5 \mathrm{wt} \% \mathrm{CaCl}_{2}$ in a water Iodide/Triiodide Electrolyte; (a) $V_{o c}$ and (b) $\boldsymbol{I}_{s c}$.

With respect to the $I_{\mathrm{sc}}$ (Figure 25(b)), when no $\mathrm{CaCl}_{2}$ was added to the electrolyte solution, RNCaSil achieved a higher $I_{\mathrm{sc}}$ than the unreinforced NCaSil (powder and cake form) and AWNCaSil, but not the EENCaSil. NCaSil in its cake form achieved a greater $I_{\mathrm{sc}}$ than the powder NCaSil, which was likely 
due to NCaSil maintaining a larger surface area when kept wet. Generally, a strong relationship between the surface area and the $I_{\mathrm{sc}}$ appeared to exist, suggesting that the NCaSil may have been acting as a surface polyelectrolyte. The EENCaSil form had the highest surface area and $\mathrm{Ca}^{2+}$ access of all the discussed NCaSils. Ethoxyethanol tends to be lost from the NCaSil when in solution, thus it was able to release previously encapsulated $\mathrm{Ca}^{2+}$. The high surface area and $\mathrm{Ca}^{2+}$ availability explain why, in a $\mathrm{CaCl}_{2}$ free electrolyte solution, the EENCaSil had the highest $I_{\mathrm{sc}}$.

To alleviate the effect of the calcium release and investigate other features of the NCaSil cells, 5 wt\% $\mathrm{CaCl}_{2}$ was added to the electrolyte solution, resulting in an increase in $I_{\mathrm{sc}}$ for all the different forms of NCaSil cells. This was likely due to the $\mathrm{Ca}^{2+}$ ions in the electrolyte increasing the overall ion mobility. With $5 \mathrm{wt} \% \mathrm{CaCl}_{2}$ added to the electrolyte, the inherent $\mathrm{Ca}^{2+}$ content of the different NCaSils was no longer the determining factor. Once the cells all had sufficient $\mathrm{Ca}^{2+}$ available, the stability of the silicate appeared to determine cell performance. Thus, RNCaSil became the dominant form, as RNCaSil was the most stable form over extended periods (days) of measurement. The EENCaSil cells did not change significantly compared to the other NCaSils due to their initially high $\mathrm{Ca}^{2+}$ availability. The second most stable NCaSil form was the acid washed, followed by the pure NCaSil and EENCaSil, the latter two of which were both relatively unstable.

Additionally, that the stability of the silicate determined cell performance, with $5 \mathrm{wt} \% \mathrm{CaCl}_{2}$ added to the electrolyte, supported the supposition that soggy sand electrolytes had been made. The retention of $\mathrm{Ca}^{2+}$ on the silicate surface would have allowed for the formation of soggy sand electrolytes and stable NCaSils would be more readily able to form such electrolytes (as discussed further below, Part IV 12).

\subsubsection{Fill Factor and Overall Power Conversion Efficiency}

NCaSil cell and iodide/triiodide photogalvanic cell curves (Figure 26) gave linear relationships in plots of their respective fill factors, which meant that the maximum power point was relatively low. The fill factors of a typical RNCaSil in a water iodide/triiodide electrolyte with $5 \mathrm{wt} \% \mathrm{CaCl}_{2}$ and the electrolyte alone were 0.27 and 0.24 respectively. The performance of the RNCaSil cell was superior to that of the electrolyte on its own. The RNCaSil cell also displayed enhanced operation stability, which, as discussed further below, was attributable to NCaSil having a greater band gap than the relevant triiodide band gap and the fact that iodine was easily photobleached, whereas NCaSil inhibited iodine transitions. 


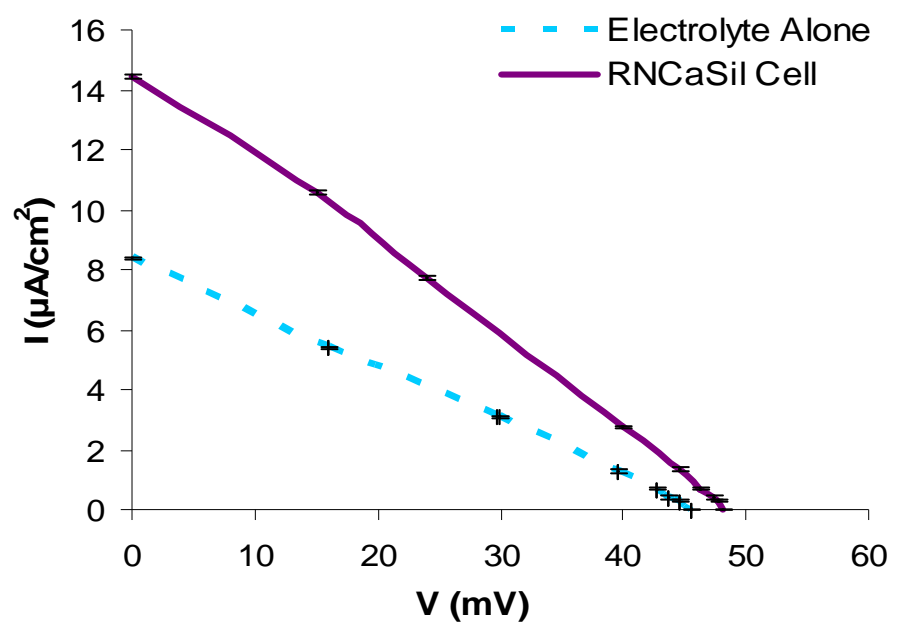

Figure 26. Current versus Voltage of Reinforced NCaSil in a Water Iodide/Triiodide Electrolyte with 5 wt\% $\mathrm{CaCl}_{2}$ $\left(0.10 \mathrm{~g}: 1.0 \mathrm{~cm}^{3}\right)$ and of The Same Electrolyte Alone.

The overall power conversion efficiencies were $8.6 \times 10^{-3} \%$ for the typical RNCaSil (in a water iodide/triiodide electrolyte with $5 \mathrm{wt} \% \mathrm{CaCl}_{2}$ ) and $4.3 \times 10^{-3} \%$ for the electrolyte alone (Figure 26). That the increase in cell performance, upon adding RNCaSil to the iodide/triiodide electrolyte, was relatively small implied that the RNCaSil was not acting as a semiconducting material, but as an insulating material in a soggy sand electrolyte when used in conjunction with the iodide/triiodide electrolyte. If the RNCaSil were acting as a semiconducting material, one would have expected to see a greater gain in cell performance.

\subsubsection{Possible Surface Area Effects}

Assuming that the NCaSils were acting as semiconducting materials, apart from the absence of additional light-absorbants/dyes, the low power conversion efficiencies observed were likely due to: (1) the disordered nature of amorphous NCaSil in general; and (2) the NCaSil not being sintered onto the photoelectrode.

As higher ordered materials tend to transport electrons more efficiently, ${ }^{[124]}$ the disordered nature of the NCaSil structure could have been unfavourable for cell performance. $\mathrm{In}_{\mathrm{TiO}_{2}}$ DSSCs, nanoparticles are sintered together onto the photoelectrode to achieve better connectivity between the particles and between the particles and substrate, to allow for efficient electron transfer. ${ }^{[28]}$ The inability to readily thermally sinter pure NCaSil particles, due to decomposition to the more thermally stable form wollastonite at $720{ }^{\circ} \mathrm{C},{ }^{[125]}$ resulted in poor inter-particle and particle-to-substrate connectivity and thus inefficient electron transfer. The plate structure of NCaSil (Figure 9) may have acerbated this in that it 
could have required a large number of hops for charges to get from their point of generation to the electrode. Hops would not only have been made between NCaSil particles, but also between the grain boundaries of the plates that made up the particles.

The difficulties in thermally sintering pure NCaSil also meant that there would not have been a layer of NCaSil covering the photoelectrode. Consequently, contact between the NCaSil and photoelectrode was poor and recombination was not inhibited, as the hole conductor and photoelectrode could easily interact.

\subsubsection{Effect of Wavelength Light Filters}

Preliminary tests to observe the effects of light filters on the cell performance of a reinforced calcium silicate in iodide/triiodide (not sensitised) indicated that calcium silicate had a wide spectral absorbance range, spreading into the visible region, meaning that, if it were acting as a semiconducting material, it could potentially be employed without the addition of a sensitiser (Figure 27(a)). This wide spectral range supported the absorbance spectra obtained from wet and dry calcium silicate, which showed absorbance over the whole spectral range as aforementioned (Part I 4.1).

Though the iodide/triiodide predominantly shows absorbances at around 350, 290 and $220 \mathrm{~nm},{ }^{[60]}$ and no absorbance peaks above $450 \mathrm{~nm}$ (see below, Figure 28), results of Stevenson et al. ${ }^{[106]}$ showed that the iodide/trriodide cell continues to function up to approximately $430 \mathrm{~nm}$, before cell performance starts to decline to approximately zero at $710 \mathrm{~nm}$. The data obtained for the RNCaSil and iodide/triiodide cell showed similar behaviour (Figure 27(b)). However, the shape of the curve observed with the RNCaSil and iodide/triiodide cell indicated that the cells did not simply exhibit the photogalvanic process of the electrolyte, as Stevenson et al. ${ }^{[106]}$ observed a concave curve relative to the origin, whereas the curve obtained with the RNCaSil cell was convex.

Therefore, the similarity between the curves obtained for RNCaSil and iodide/triiodide cell and electrolyte alone suggested that the iodide/triiodide photogalvanic process dominated even when RNCaSil was present, rather than the RNCaSil acting as a semiconductor, whether in the DSSC or photogalvanic capacity. If the RNCaSil were acting as a semiconductor, one would have expected the behaviour of the cells containing RNCaSil to be more distinct from those only consisting of the iodide/triiodide couple. That performance increased on adding RNCaSil to the iodide/triiodide, but the 
cell exhibited a different power-over-wavelength curve shape, suggested the formation of a soggy sand electrolyte.
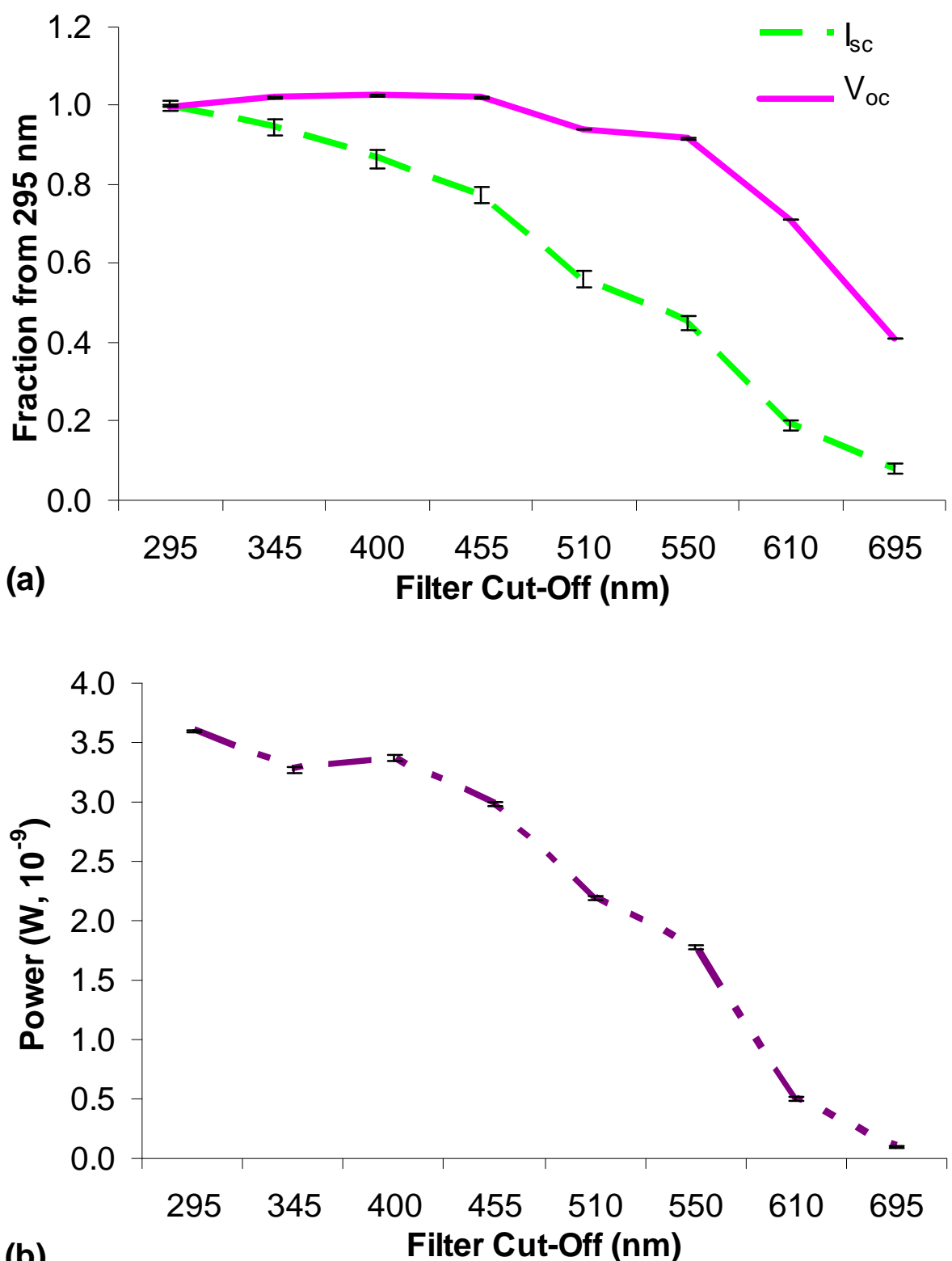

Figure 27. Effect of Light Filters on the Reinforced Calcium Silicate Cell in Ethanol Iodide/Triiodide: (a) $V_{o c}$ and $I_{s c}$; (b) Power.

\subsubsection{Spectra}

\subsubsection{Infrared Spectroscopy}

IR investigation of RNCaSil alone and with iodide/triiodide electrolytes showed that there was little difference in the spectra whether the electrolyte was present or not, whether $\mathrm{CaCl}_{2}$ was added to the 
electrolyte and what solvent was used for the electrolyte (see Appendix, Part VII 1, Figures 98 to 100). In all the spectra, large Si-O-H or $\mathrm{H}_{2} \mathrm{O}$ stretches $\left(1430 \mathrm{~cm}^{-1}\right)$, bicarbonate and carbonate stretches (1630 and $1450 \mathrm{~cm}^{-1}$, respectively $)^{[126]}$ and $\mathrm{Si}-\mathrm{O}$ stretches $\left(1045 \mathrm{~cm}^{-1}\right)$ were found. The only differences in the spectra obtained could be attributed to the solvents used (the effect on the cell performance of which is discussed below, Part IV 4.4). For example, in ethylene glycol, the sample's IR spectrum showed stretches at $2496 \mathrm{~cm}^{-1}$ and $2880^{-1}$, representing the $\mathrm{sp}^{3}-\mathrm{C}$ in the solvent, and a C-O stretch at $1086 \mathrm{~cm}^{-1}$. Similar differences were found in the IR spectrum of a sample solvated in 2-ethoxyethanol.

\subsubsection{UV-visible Spectra}

The UV-vis also showed little difference between different iodide/triiodide electrolyte formulations alone or when with RNCaSil. A water-based iodide/triiodide electrolyte showed absorbances at 348, 288 and $221 \mathrm{~nm}$ (Figure 28). The two highest peaks represented triiodide and the peak at $221 \mathrm{~nm}$ iodide. $^{[60]}$ There was no peak (at $500 \mathrm{~nm}$ ) for iodine, ${ }^{[60]}$ which was not surprising given that one expected all (or most) of the iodine to be turned into triiodide. The highest peak was shifted slightly higher with the addition of RNCaSil to $353 \mathrm{~nm}$. A $70 \%$ ethanol iodide/triiodide electrolyte exhibited higher triiodide absorbances, at 360 and $292 \mathrm{~nm}$, which remained unchanged when with RNCaSil.

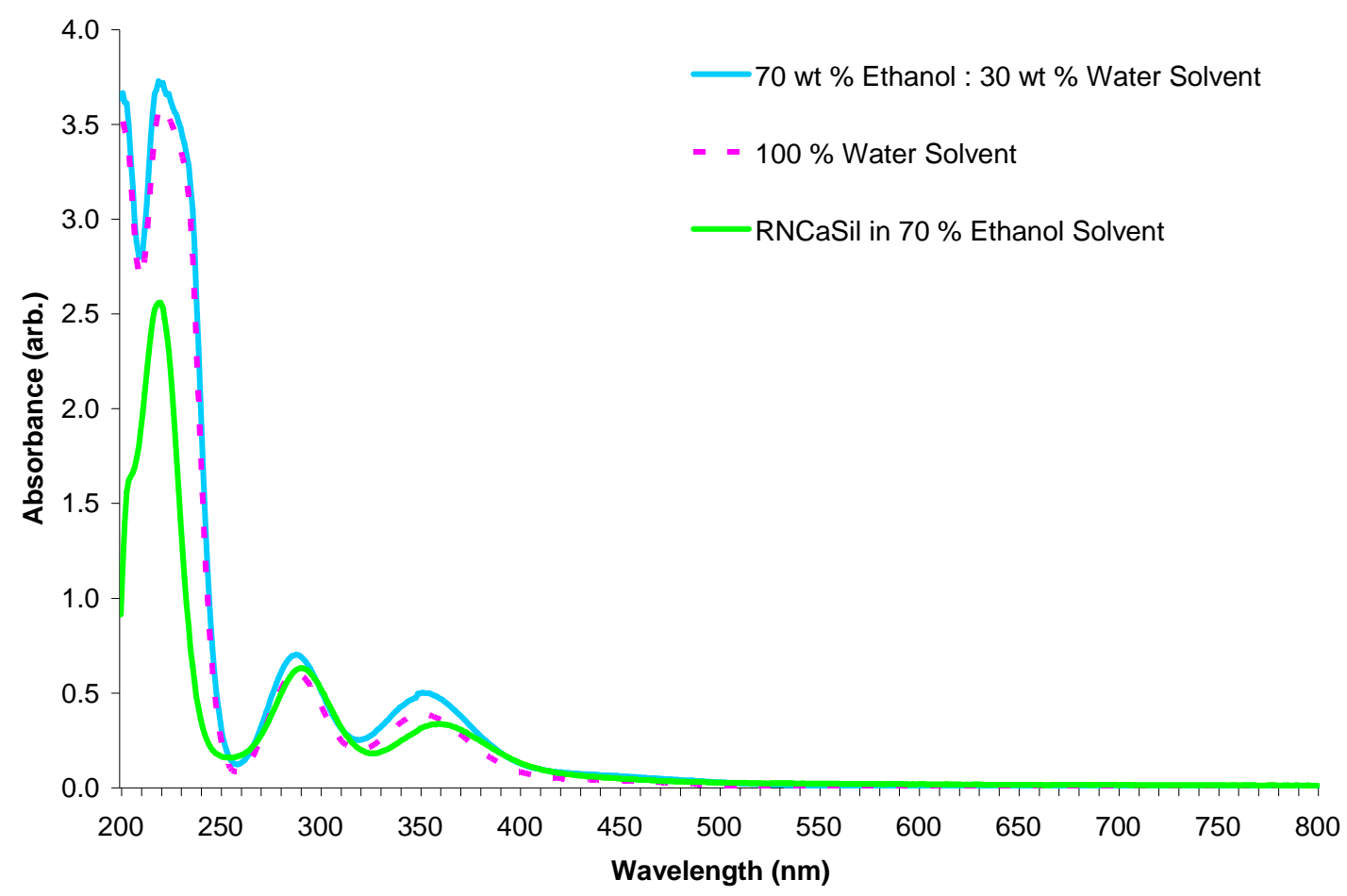

Figure 28. UV-vis of the Water-Based and 70\% Ethanol $30 \%$ Water Iodide/Triiodide Electrolytes, and RNCaSil with $100 \%$ Water-Based Iodide/Triiodide Electrolyte. 


\subsubsection{Cyclic Voltammetry}

Cyclic voltammograms of the water iodide/triiodide electrolyte were collected, with and without the addition of $\mathrm{CaCl}_{2}$, and for RNCaSil or NCaSil based systems (Figure 29(a)).
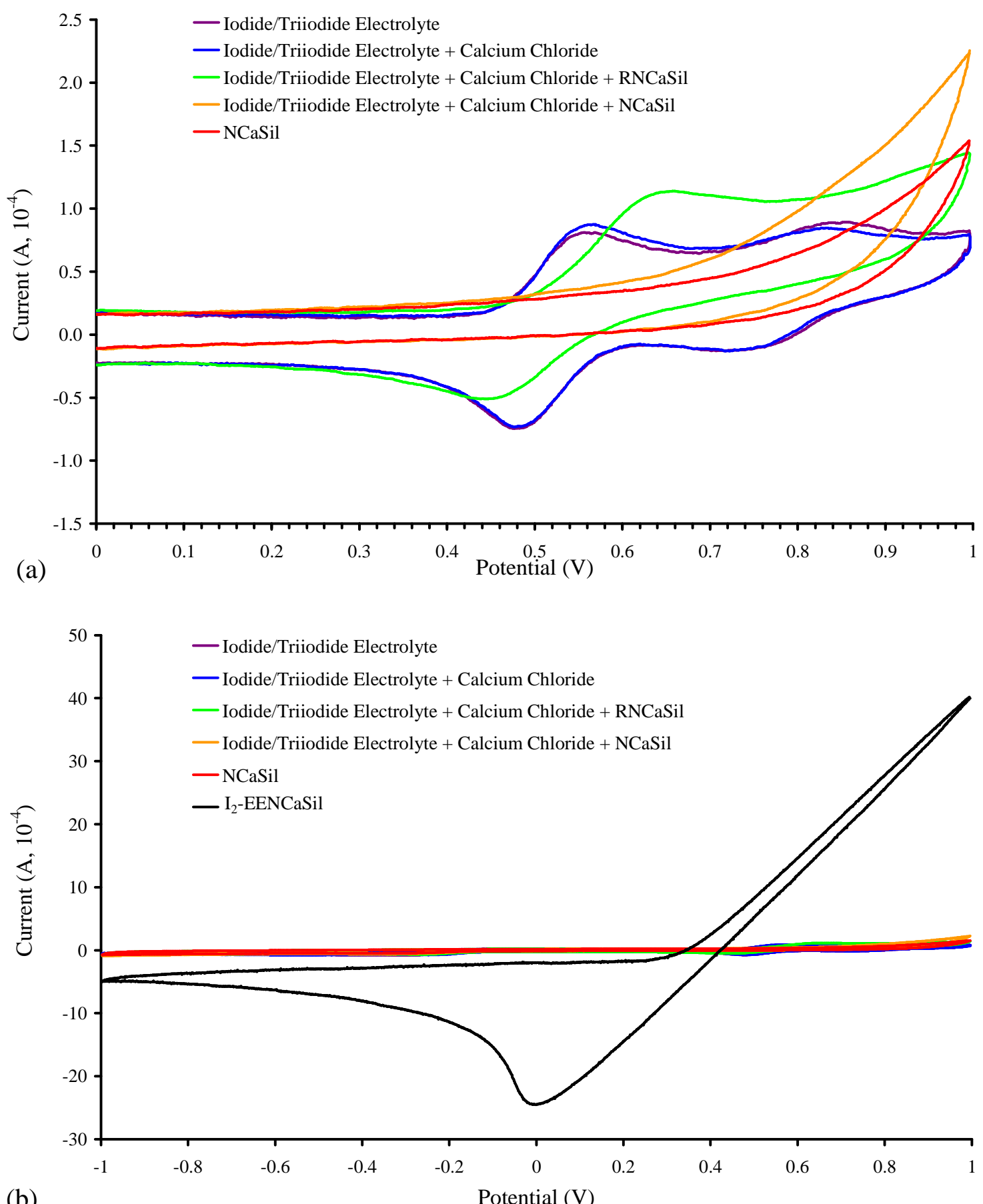

(b)

Figure 29. Cyclic Voltammetry of: (a) Water Iodide/Triiodide Electrolyte with Calcium Chloride and NCaSils; and (b) $\mathbf{I}_{2}$-EENCaSil. 
Little difference was observed with the electrolyte alone, whether or not $\mathrm{CaCl}_{2}$ was present. This was not surprising, given that $\mathrm{Ca}^{2+}$ is difficult to reduce $\left(E^{\theta}=-2.87 \mathrm{~V}\right)^{[127]}$ and should not have contributed to the processes observed in the potential range tested. The reversible oxidation peaks observed around $0.52 \mathrm{~V}$ represented the iodide/triiodide transitions $\left(3 \mathrm{I}^{-} \leftrightarrow \mathrm{I}_{3}{ }^{-}+2 \mathrm{e}\right)^{[128]}$ and the peaks around $0.79 \mathrm{~V}$ the triiodide/iodine transition $\left(2 \mathrm{I}_{3}{ }^{-} \leftrightarrow 3 \mathrm{I}_{2}+2 \mathrm{e}\right) .^{[128]}$

The cyclic voltammogram of the electrolyte with RNCaSil and NCaSil were particularly interesting because they showed the effect that the NCaSils had on the electrolyte's transitions. The presence of RNCaSil shifted the $0.52 \mathrm{~V}$ transition to $0.54 \mathrm{~V}$, lowered its intensity, and completely removed the transition at $0.79 \mathrm{~V}$. The shift of the iodide/triiodide transition to a more positive potential explained why RNCaSil cells tended to have slightly higher $V_{o c}$ than the electrolyte alone. Furthermore, the decrease in peak intensity rationalised why the current of cells often decreased on the addition of RNCaSil to the iodide/triiodide electrolyte (see below, Part IV 4.1), in the absence of other factors (such as ratio and $\mathrm{CaCl}_{2}$ content).

On testing NCaSil with the electrolyte, both transitions were removed and the voltammogram was more like that of NCaSil by itself than when with the iodide/triiodide electrolyte; the NCaSil suppressed the iodide/triiodide/iodine transitions more than the RNCaSil. The additional suppression of $3 \mathrm{I}^{-} \leftrightarrow \mathrm{I}_{3}{ }^{-}+2 \mathrm{e}$ was why NCaSil was less efficient than RNCaSil when used in conjunction with the redox couple, as the latter allowed this process to take place. The transition between iodide and triiodide was required for the photoactive process to take place. The formation of iodine was not important for the cell process and, thus, its inhibition by RNCaSil or NCaSil should not have affected cell efficiency.

It was suspected that the suppression of these transitions was related to the interaction of iodine with the NCaSils. If this was the case, then the NCaSil inhibiting the transitions was expected, as it has previously been observed that iodine interacted more readily with the surface of NCaSil than RNCaSil. ${ }^{[115]}$ The suppression of iodine transitions on the interaction of iodine with NCaSil was supported by the voltammogram of $\mathrm{I}_{2}$-treated EENCaSil (Figure 29(b)). This also showed a complete lack of the transitions observed with the iodide/triiodide electrolyte. The impediment of the iodide/triiodide and iodine transitions, by different NCaSils, further explained why photobleaching was limited in NCaSil-containing cells. 
It was not clear what the irreversible transition at $0 \mathrm{~V}$ represented. The $\mathrm{H}^{+} / \mathrm{H}^{2}$ transition occurs at this potential, however it usually requires a catalyst, such as platinum, and is reversible.

\subsection{Vanadium- and Cerium- Doped NCaSils}

Of particular interest were the transition metal vanadium- and lanthanide cerium-treated NCaSils, as these metals had been shown to improve NCaSil activity in other applications. ${ }^{[11]}$ As with the other NCaSils, there was no visible difference in $V_{o c}$ between the two metal-modified samples compared to the other NCaSils (Figure 30(a)). The fill factors for these modified NCaSils were similar to those of the RNCaSil cell, between 0.24 and 0.27 (Part IV 1.1.1), with linear current over voltage relationships. However, the cell efficiencies for the V- and Ce-containing cells were greater, due to a marked difference in the currents obtained for these samples (Figure 30(b)).

The V-NCaSil cell's performance, even without any $\mathrm{CaCl}_{2}$ added to the electrolyte, showed a greater $I_{s c}$ than the RNCaSil cell with $5 \mathrm{wt} \% \mathrm{CaCl}_{2}$ added, and the Ce-NCaSil cell yielded comparable values to the said RNCaSil cell. The overall power conversion efficiencies for the $\mathrm{V}$ - and Ce-treated NCaSil cells were $7.6 \times 10^{-3} \%$ and $6.2 \times 10^{-3} \%$, respectively, without any $\mathrm{CaCl}_{2}$ addition. As with the other NCaSils, the cell efficiencies of the V-and Ce-treated NCaSil cells increased upon addition of 5 wt\% $\mathrm{CaCl}_{2}$ to the electrolyte, due to the enhanced current. The $I_{s c}$ for the V-NCaSil cells increased to over twice that exhibited by comparative RNCaSil cells, and the Ce-NCaSil cell's $I_{s c}$ was nearly double that of the RNCaSil cells. The improvements were similar in proportion to the increases observed for the other NCaSils.

The overall power conversion efficiency for the $\mathrm{CaCl}_{2}$-containing RNCaSil cell was $4.3 \times 10^{-3} \%$, that for the V-treated NCaSil cell was $15.2 \times 10^{-3} \%$, and that for the Ce-treated NCaSil cell was $13.8 \times 10^{-3}$ $\%$. Energy dispersive spectrometry (EDS) undertaken with the two treated NCaSils indicated that that vanadium-content of V-NCaSil was around $2 \%$. However, in Ce-NCaSil, the cation additive was below the detection limit for this element and method of analysis and, therefore, no cerium was detected. That cerium was present was not doubted, as the silicate samples were visibly intensively yellowed by its presence. As the samples otherwise show similar silicon, calcium and oxygen contents, the higher vanadium levels could have been why V-NCaSil showed greater cell performance than CeNCaSil. Alternatively, it may have been due to the vanadium source being an anion (vanadate), whereas as cerium was as a cation $\left(\mathrm{Ce}^{2+}\right)$. As an anion, it would not have been well bound to the 
anionic surface of NCaSil, thus allowing it to be released into the electrolyte more readily, increasing the ionic atmosphere of the sample.
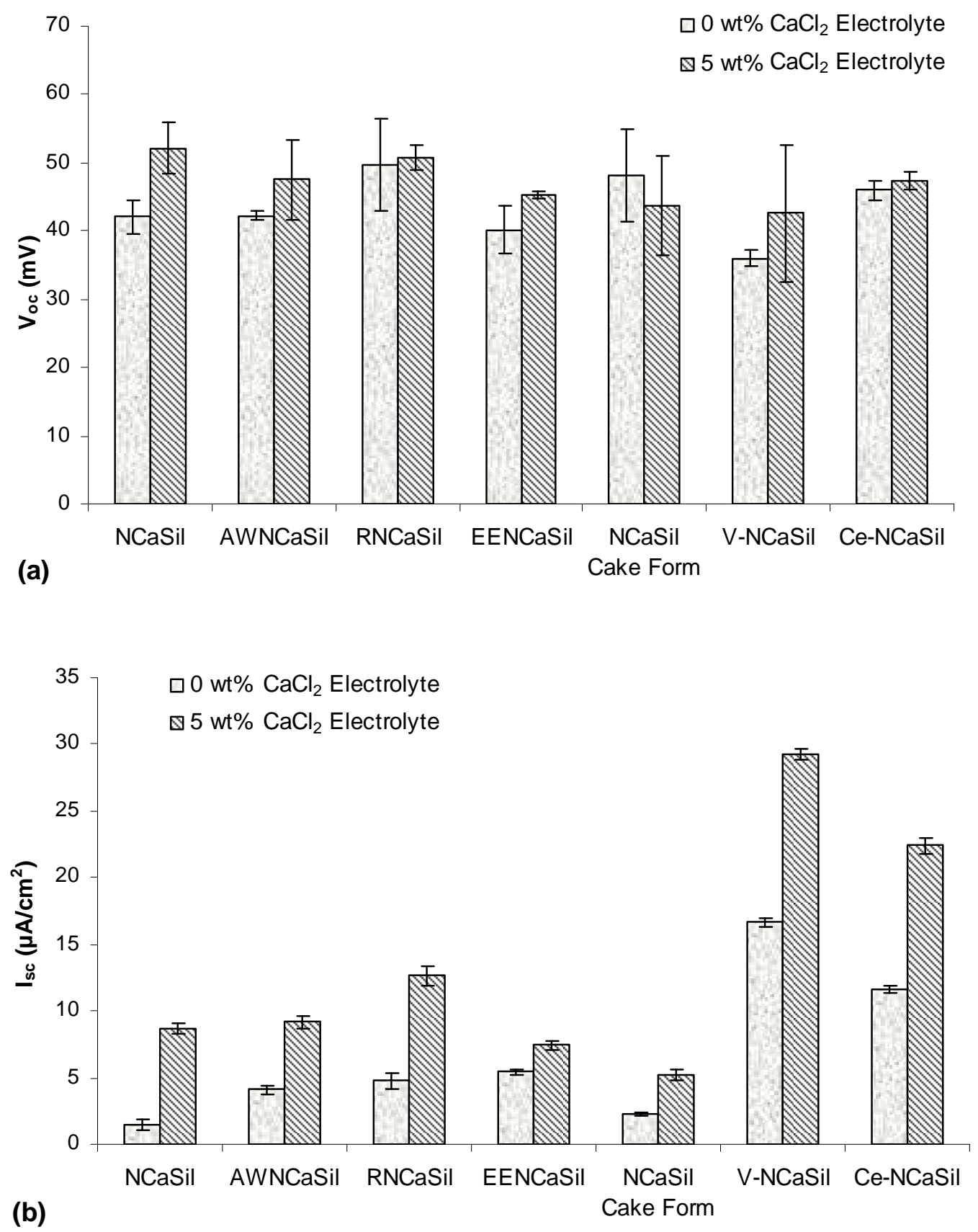

Figure 30. V- and Ce-Treated NCaSil; (a) $V_{o c}$ and (b) $I_{s c}$.

$\mathrm{I}_{2^{-}}$and Ru-treated EENCaSil were also tested, though (due to material constraints) in a different capacity in beaker cells (Experimental, Part III 6), and thus were not directly comparable to these VNCaSil and Ce-NCaSil results. However, they are mentioned here briefly for comparison. From the 
long-term testing, the $\mathrm{I}_{2}$-EENCaSil showed poor performance. Initially, the $V_{o c}$ and $I_{s c}$ observed were higher than those of equivalent $\mathrm{V}-\mathrm{NCaSil}$ and Ce-NCaSil cells. However, over time, both the $V_{o c}$ and $I_{s c}$ dropped off to become similar to the initial data obtained for RNCaSil. The Ru-EENCaSil also exhibited high initial perfomance, with a voltage over $100 \mathrm{mV}$ and current ten times higher than equivalent V-NCaSil and Ce-NCaSil cells and over six times higher than that of RNCaSil. However, this cell proved quite unstable, with the $V_{o c}$ and $I_{s c}$ decaying over time. These findings are discussed in more depth below (Part IV 8.5).

\subsection{Notes on the Synthesis of NCaSils}

\subsubsection{Preparation of RNCaSil}

After an initial batch of RNCaSil had been used up, a second batch was made and tested. This second batch did not seem as efficient in cell, as its currents were markedly lower (Figure 31). Interestingly, only the current was affected, not the voltage, indicating that the band gaps of the batches were either not greatly changed or did not significantly impact cell performance. Results already discussed (Part IV 1.1) suggested the latter. A third and fourth batch were made with similar results. It was not until a fifth RNCaSil batch was made that currents of comparable levels to the initial batch were achieved. The main components of NCaSil were sodium silicate $(\mathrm{NaSil})$ and $\mathrm{Ca}(\mathrm{OH})_{2}$. The only difference between Batches \#2, 3 and 4 and Batch \#5 was that, in the latter, the NaSil solution was added to a rapidly stirring $\mathrm{Ca}(\mathrm{OH})_{2}$ solution, rather than the reverse. The order of addition would have affected the relative concentrations of the components during particle formation. Adding $\mathrm{NaSil}$ to $\mathrm{Ca}(\mathrm{OH})_{2}$ resulted in a mixture that was initially $\mathrm{Ca}(\mathrm{OH})_{2}$ rich and NaSil poor. This must have, to some degree, affected the exact structure formed and thus the cell performance of the samples.

Particle size did not appear to be affected by the differences in mixing order of $\mathrm{NaSil}$ and $\mathrm{Ca}(\mathrm{OH})_{2}$ solutions. In either case, the main particle size was approximately $1 \mu \mathrm{m}$ (Figure 32 ). It was suspected that the reason why the initial RNCaSil batch (Batch \#1) exhibited a slightly different and wider particle distribution was due to its relative age when the analysis took place, because of structure collapse over time. Additionally, surface area measurements of the different RNCaSils did not indicate any particular variance dependant on the order of mixing the different components.

However, energy dispersive spectrometry (EDS) showed that, relative to silicon, when the NaSil solution was added to the $\mathrm{Ca}(\mathrm{OH})_{2}$, rather than the other way around, the calcium content of the 
material obtained was almost three times as great, but the oxygen content was lower. Therefore, the differences in cell performance of these RNCaSils was attributed to the variances in $\mathrm{Ca}^{2+}$ content.
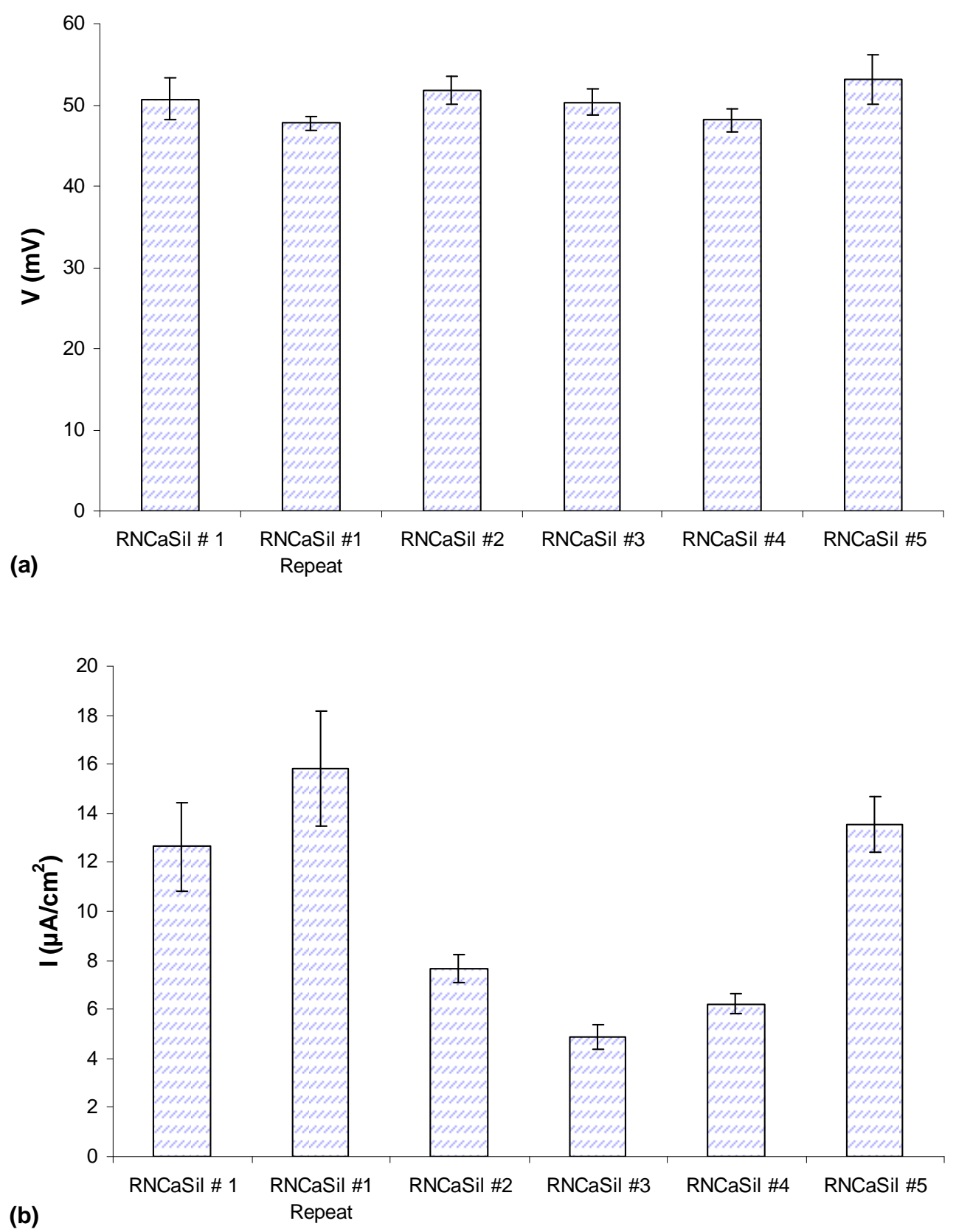

Figure 31. Different RNCaSil Batches. Tested in a Normal Gasket with a C-coated Back Electrode; $0.10 \mathrm{~g}$ RNCaSil: $1.0 \mathrm{~cm}^{3}$ Water Iodide/Triiodide $\left(5 \mathrm{wt} \% \mathrm{CaCl}_{2}\right.$ ) Electrolyte; a) $V_{o c}$ and (b) $I_{s c}$.

During the NaSil addition to $\mathrm{Ca}(\mathrm{OH})_{2}$, the relative concentration of $\mathrm{Ca}(\mathrm{OH})_{2}$ would have been greater than that of NaSil, resulting in more $\mathrm{Ca}^{2+}$ on the surface of the silicate particles and thus greater $\mathrm{Ca}^{2+}$ accessibility, both with regards to the EDS method used (with a relatively low penetration depth) and when used in cells with iodide/triiodide. Greater surface-Ca ${ }^{2+}$ content resulting in better cell 
performance was also supported by data already discussed (Part IV 1.1) and is discussed further below (Part IV 4.3.1 and 9.6).

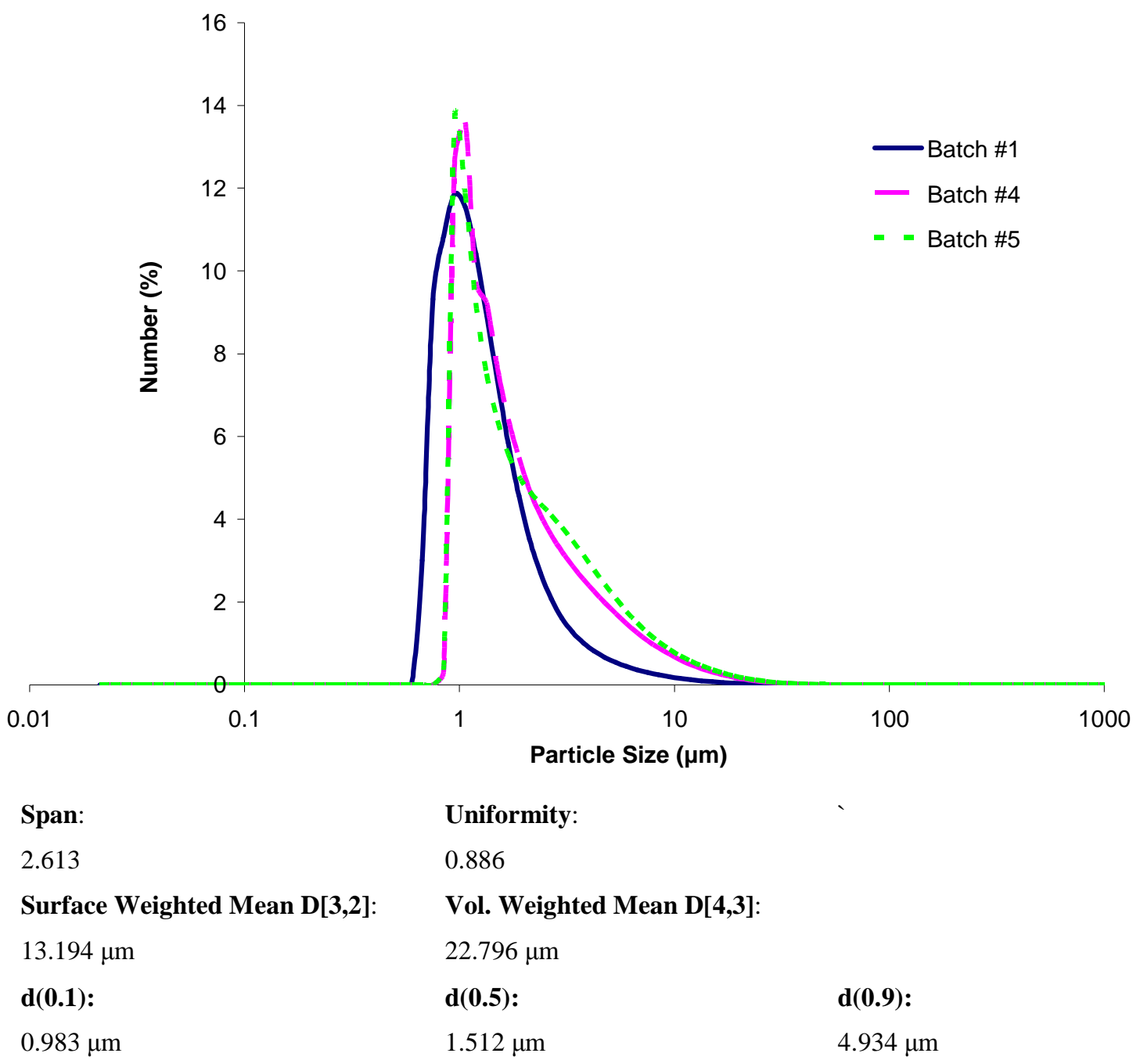

Figure 32. Particle Size Distribution Analysis of Three RNCaSils (Filter Cakes): Sodium Silicate Solution Added to the Calcium Hydroxide Solution in Batches \#1 and 5; and Calcium Hydroxide Solution Added to the Sodium Silicate Solution in Batch \#4.

The different order of mixing the two components of RNCaSil also affected whether a gel could be formed from the RNCaSil, which is discussed in detail below (Part IV 8.2.1).

\subsubsection{The Consistency of Continuous Method RNCaSil}

Continuous method RNCaSil (CM-RNCaSil) is reinforced NCaSil prepared in a continuous-flowstirred-tank reactor (CSTR), rather than in a batch reactor. Batch reactors are used for small scale 
reactions, wherein the reactants can be added simultaneously or sequentially (the latter in the case of RNCaSil) and stay in the reactor for a fixed time before the product is removed. Continuous method reactors differ in that reactants and products are continuously added and removed (Experimental, Part III 1.5).

The results shown (Figure 33) were from the same batch of CM-RNCaSil. The wide range of cell efficiencies observed with CM-RNCaSil (compared to the much more consistent RNCaSil) was related to the method of manufacture. With batch RNCaSil, individual batches were normally very selfconsistent, i.e., there was uniformity within the batch. However, from batch to batch, differences were observed, particularly in particle size and surface area. A CM-RNCaSil sample could be viewed as consisting of many batches within one mixture. For the flow-stirred method used, the reactants/products had a mean residence time in the reactor, determined by the flow rate and reactor volume. This mean residence time meant that some reactants/products could have entered the vessel and left quickly, while others may have been in the vessel for longer. Thus, there was potentially a wide range of residence times and even some un-reacted reactants exiting the vessel.

If one sample of CM-RNCaSil is considered as consisting of many "mini-batches", then there was more consistency between the mini-batches in CM-RNCaSil compared to between batches of non-CMRNCaSil. However, because the mini-batches were not completely uniform, within the one batch of CM-RNCaSil, there were differences in particle size and surface area. Consequently, there were great variances in cell-performance from the same CM-RNCaSil sample.

Each of the results shown in Figure 33 is representative of three cells prepared from the same batch of CM-RNCaSil, i.e., $0.01 \mathrm{~g}$ of CM-RNCaSil and $1.0 \mathrm{~cm}^{3}$ of iodide/triiodide were mixed, and the three repeated cells made up from this one sample. That there were mini-batches within a single batch of CM-RNCaSil was supported by these results, as, though there were large differences between the different samples, there was very little uncertainty within each result peak.

Particle size distribution measurements were undertaken on three samples from different areas of the same batch of CM-RNCaSil (Figure 34). This clearly showed that the particle size distribution differed between the mini-batches within one batch of CM-RNCaSil. The peak particle size of the first sample was around $1 \mu \mathrm{m}$ (typical of reinforced NCaSils), whereas the other two samples exhibited peak particle sizes around $3.4 \mu \mathrm{m}$. The larger particles remained even after sonication. 
Surface area measurements performed on different areas of the same batch of CM-RNCaSil did not show any variance outside of the expected experimental uncertainty. Hence, the differences in cell performance of mini-batches within a single batch of CM-RNCaSil were credited to inconsistencies in particle size rather than surface area. It thus appeared that particle size had more of a controlling effect on cell performance than surface area for NCaSils.

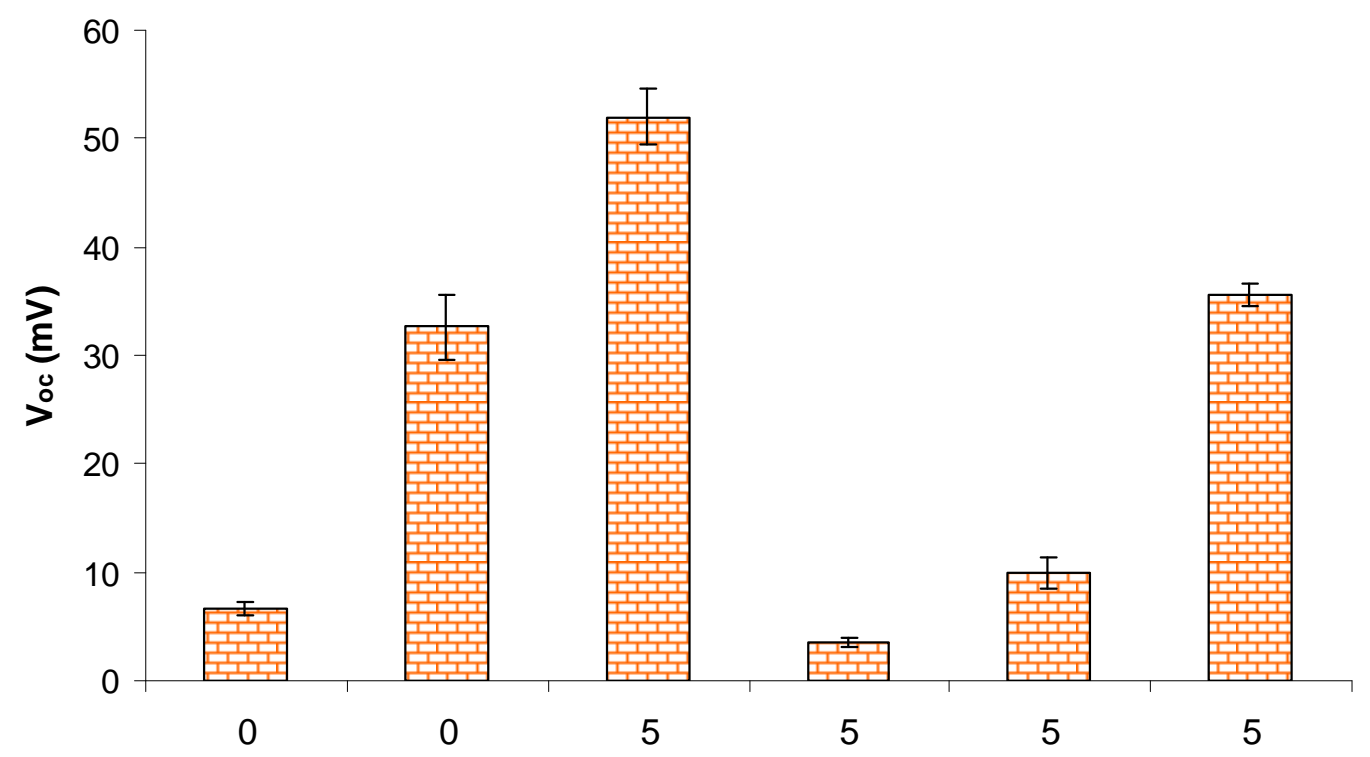

(a)

Electrolyte $\mathrm{CaCl}_{2}$ Content (wt\%)

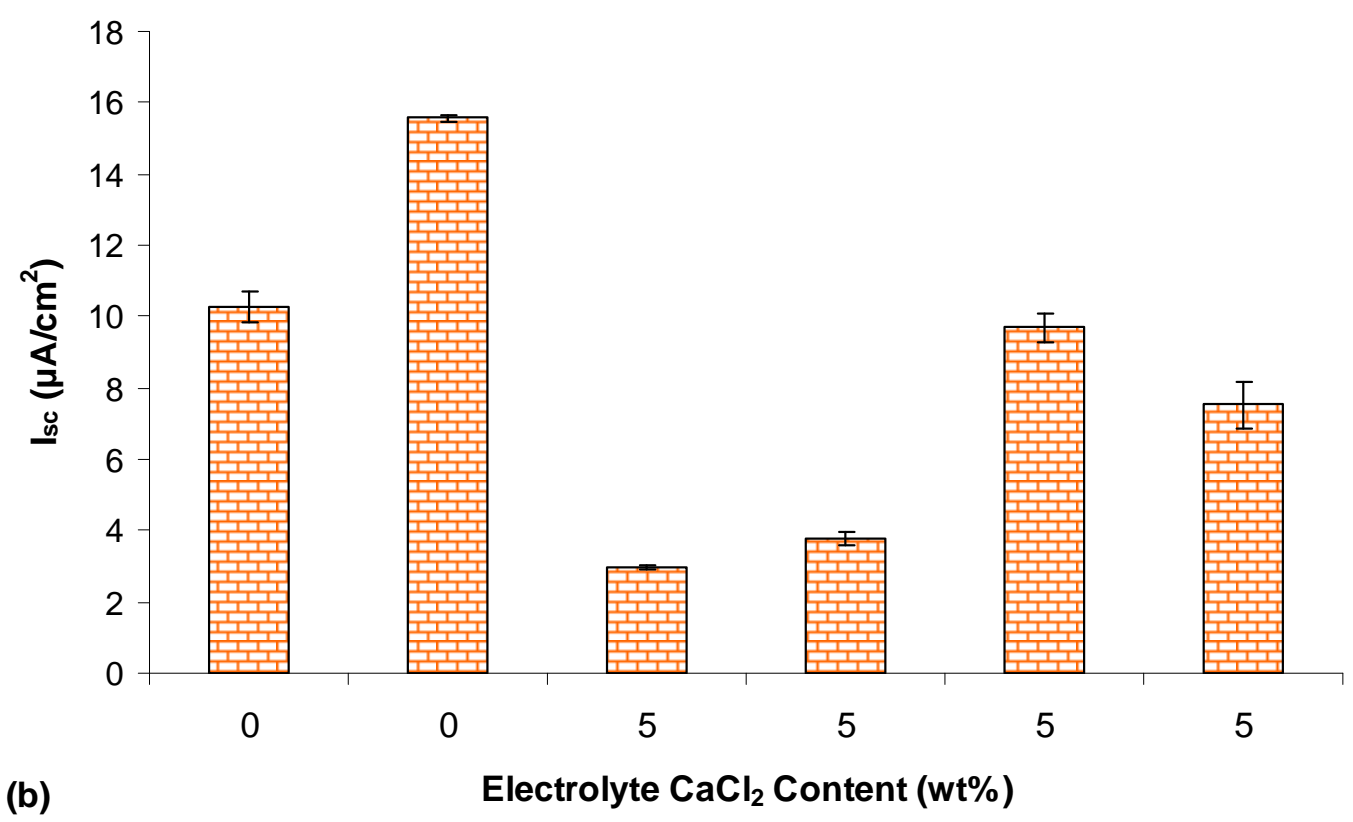

Figure 33. CM-RNCaSil in Water Iodide/Triiodide Electrolyte, with and without $5 \mathrm{wt} \% \mathrm{CaCl}_{2} ;\left(\right.$ a) $V_{o c}$ and (b) $I_{s c}$. 


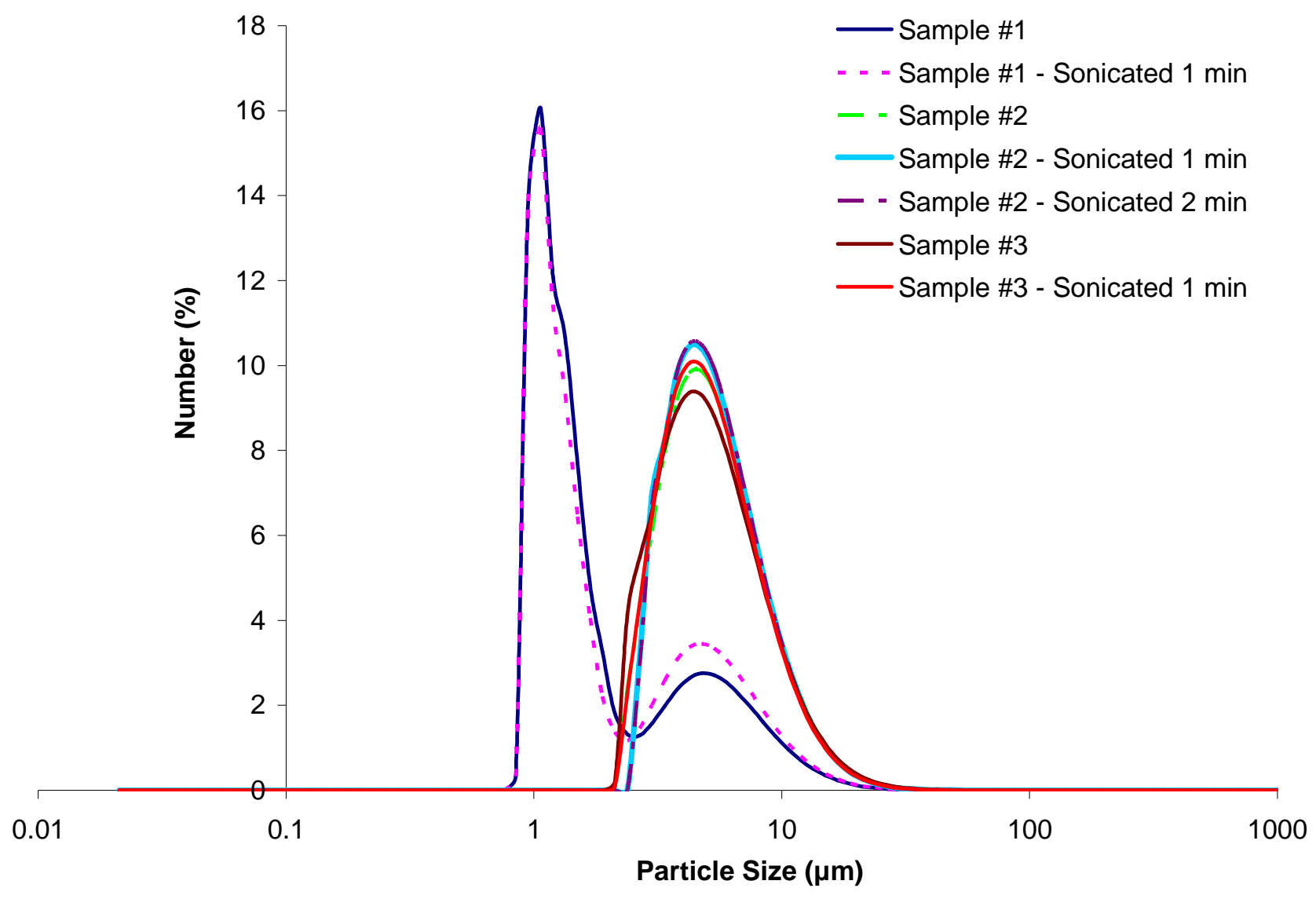

Span: Uniformity:

1.347

0.433

Surface Weighted Mean D[3,2]:

Vol. Weighted Mean D[4,3]:

$10.673 \mu \mathrm{m}$

$15.346 \mu \mathrm{m}$

d(0.1):

$\mathrm{d}(\mathbf{0 . 5})$ :

d(0.9):

$3.006 \mu \mathrm{m}$

$4.960 \mu \mathrm{m}$

$9.686 \mu \mathrm{m}$

Figure 34. Particle Size Distribution Analysis of Three Different Areas of the Same Batch of CM-RNCaSil (Powder).

Also interesting, with regards to CM-RNCaSil, was that it was the only NCaSil that showed a loss of current when $5 \mathrm{wt} \% \mathrm{CaCl}_{2}$ was added to the electrolyte solution. Why this was the case remained unsolved. Speculatively, it could have been due to the interaction of $\mathrm{CaCl}_{2}$ with un-reacted reactants, which had a residence time of almost zero in the reactor during sample preparation. 


\section{Effect of Light Intensity Filters}

Optical density filters (Experimental, Part III 16) were used on a RNCaSil-containing cell to assess the quality of the cell setup in general and specifically the light source used. Findings were hoped to also indicate whether the cell would function efficiently at lower light intensities. As can be observed from Figure 35 , both the current and voltage decreased as the intensity of light was lowered. The $I_{s c}$ decreased in quite a linear fashion, whereas the $V_{o c}$ did not; only the initial decline was linear. At a light intensity factor of 0.4 , the voltage started to fall off more rapidly with decreasing light intensity. Overall, for an intensity of $10 \%$ compared to that of an unfiltered cell the voltage decreased only by 50 $\%$ and the current by $75 \%$. This indicated that the cell performance of the NCaSil cells in the used setup was underestimated by a factor of approximately two.

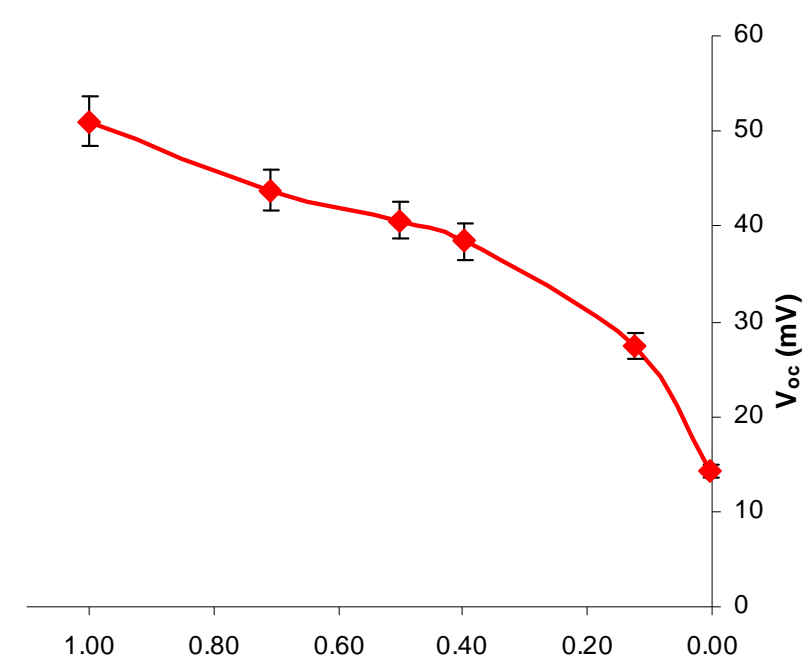

(a)

Light Intensity

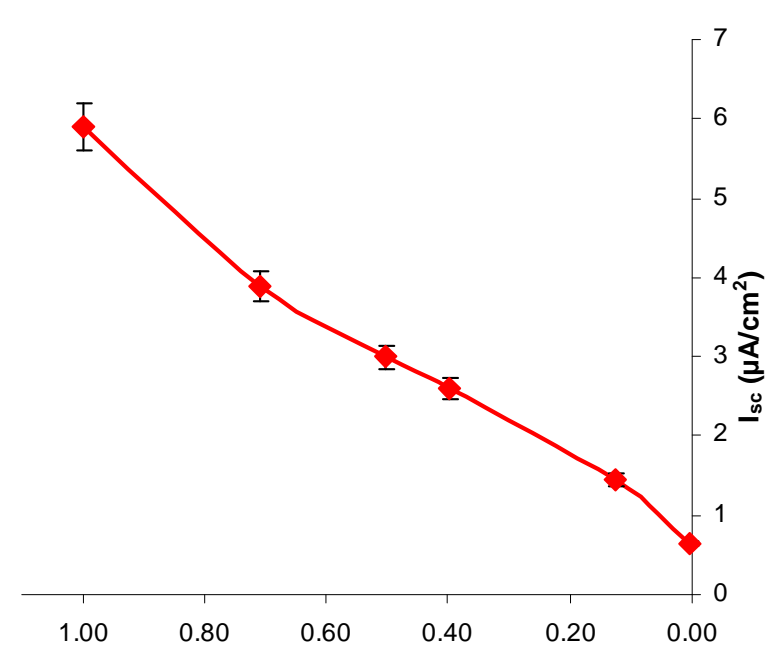

(b)

Figure 35. Effect of Light Intensity of the Cell Performance of RNCaSil in $70 \%$ Ethanol Iodide/Triiodide Electrolyte $\left(0.10 \mathrm{~g}: 1.0 \mathrm{~cm}^{3}\right)$, Pt-coated back electrode in normal gasket; (a) $V_{o c}$ and (b) $I_{s c}$.

However, the UV-vis spectrum of the optical density filters used (Figure 36) showed that, though the optical density filters did not filter out any light above $400 \mathrm{~nm}$, they blocked out almost all light around the main spectral absorbance range of NCaSil (around $270 \mathrm{~nm}$, Part I 4.1) and the iodide/triiodide electrolyte (Part IV 1.1.3 and 1.1.4.2). Thus, although NCaSil and the electrolyte showed absorbance over the whole spectral range, these particular optical filters prevented any light getting through which the un-sensitised cells predominantly required to be active. Thus, it was likely that the performance of NCaSil cells, plotted in Figure 35, were underestimated even further than surmised above. 


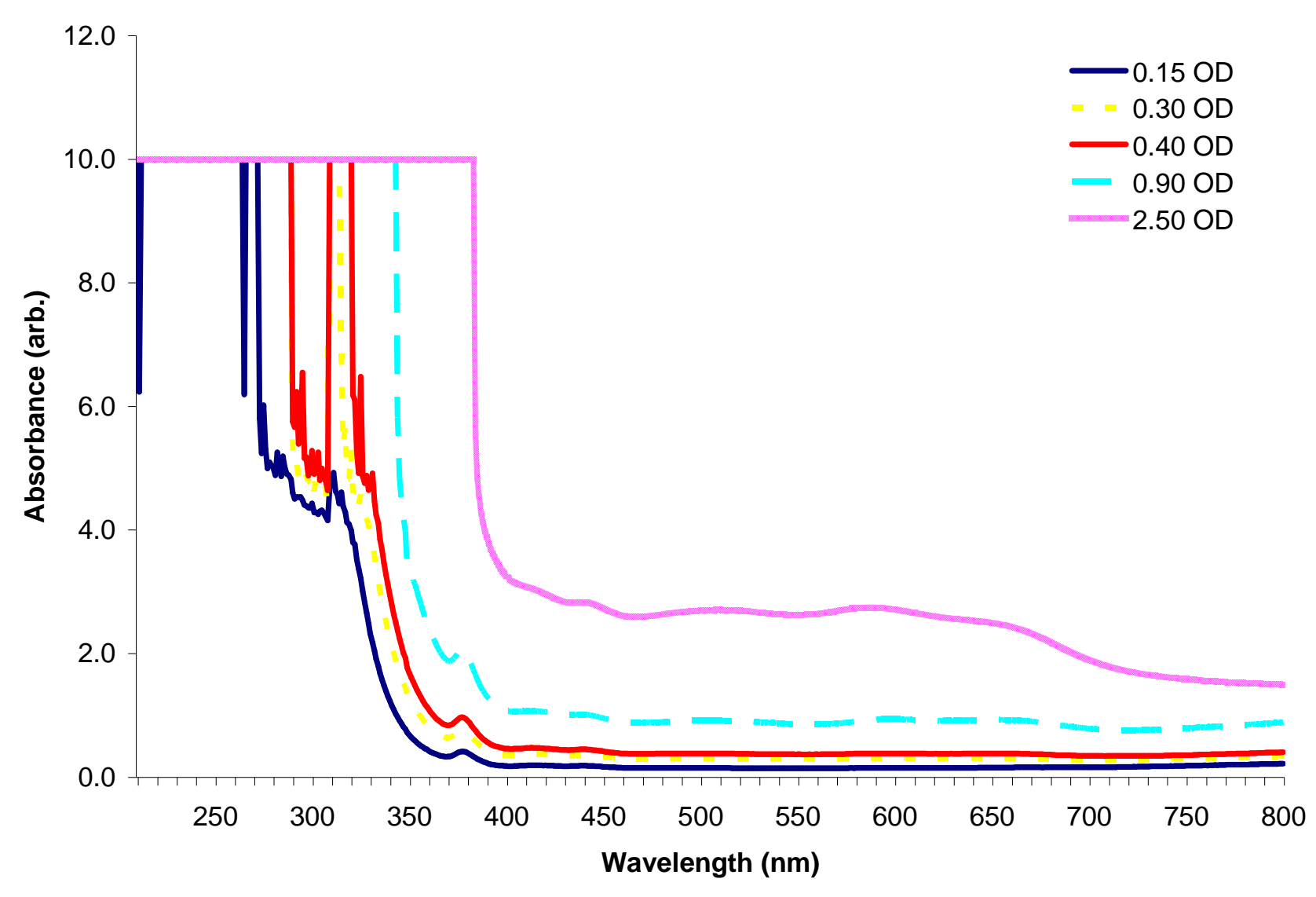

Figure 36. UV-vis of the Optical Density Filters.

The loss of functionality on decreasing the light intensity raised the question whether these cells would be viable under an ambient light source. Figure 37 shows the irradiance spectrum of the arc lamp used in this project (set to an optical intensity of $21.47 \mathrm{~W} / \mathrm{m}^{2}$ ). If one compared this to the spectral irradiance from the sun (Figure 38), it could be seen that the research lamp not only delivered light at a much wider spectral range, it also did so at a much higher intensity. From this, it could have been concluded that NCaSil cells would not have a great cell performance under relatively lower-intensity natural daylight.

Testing under natural sunlight confirmed this. A cell comprised of RNCaSil and $70 \mathrm{wt} \%$ ethanol iodide/triiodide achieved only a $V_{o c}$ of $31.6 \pm 1.6 \mathrm{mV}$ and $I_{s c}$ of $1.5 \pm 0.1 \mu \mathrm{A} / \mathrm{cm}^{2}\left(1: 00 \mathrm{pm}, 24^{\text {th }}\right.$ June 2009), compared to $74.1 \pm 0.8 \mathrm{mV}$ and $9.2 \pm 0.5 \mu \mathrm{A} / \mathrm{cm}^{2}$, respectively, for an equivalent cell tested under the research lamp. However, some consideration must be given to the fact that this was performed in the middle of winter. Furthermore, the loss of only $43 \%$ potential and $84 \%$ current was proportionately much smaller than the decrease in light intensity going from the research arc lamp to the ambient-winter light. 


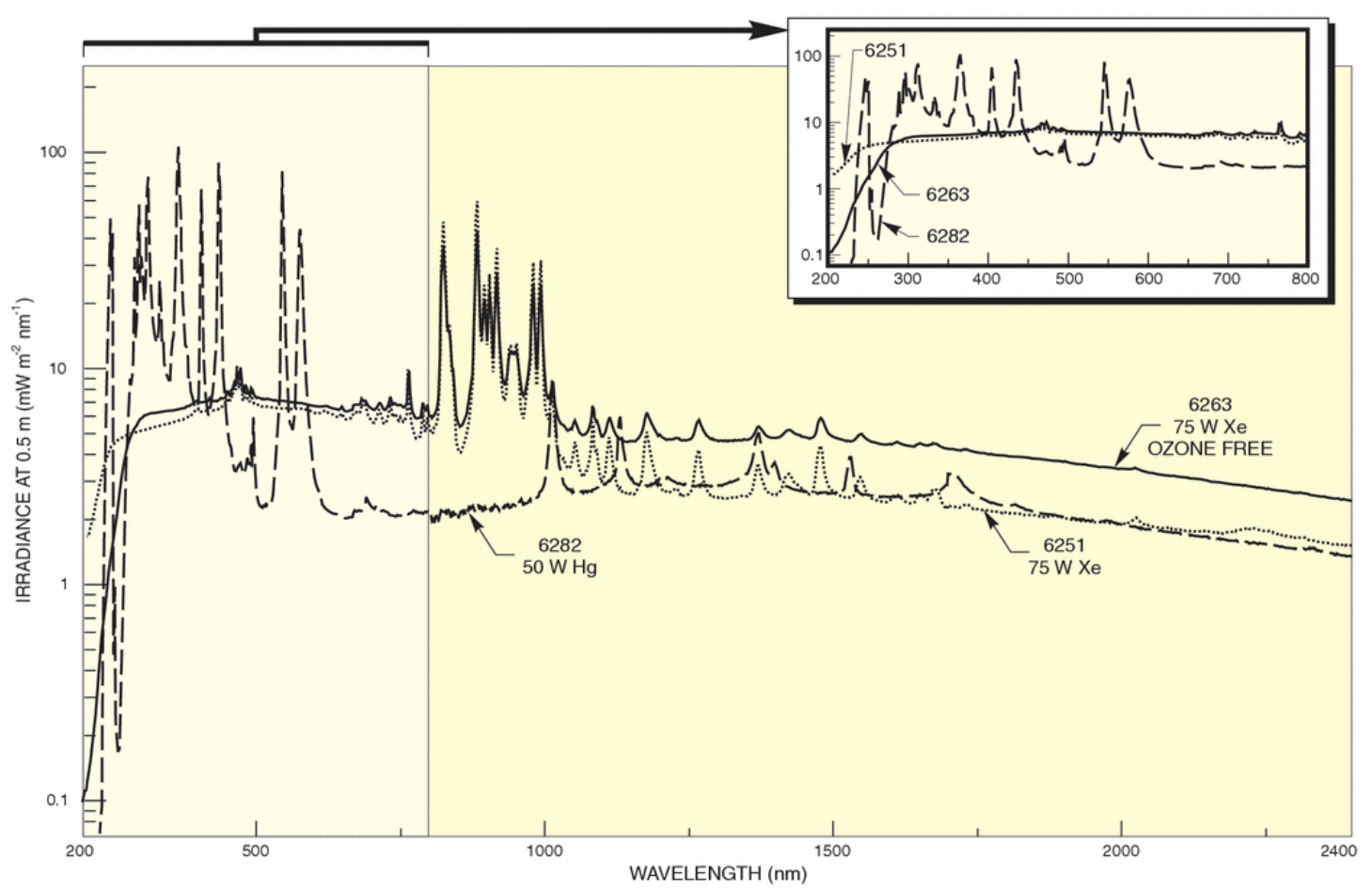

Figure 37. Irradiance from the Ozone-Free, Xenon-Arc Discharge Lamp Used, from 0.5 m.

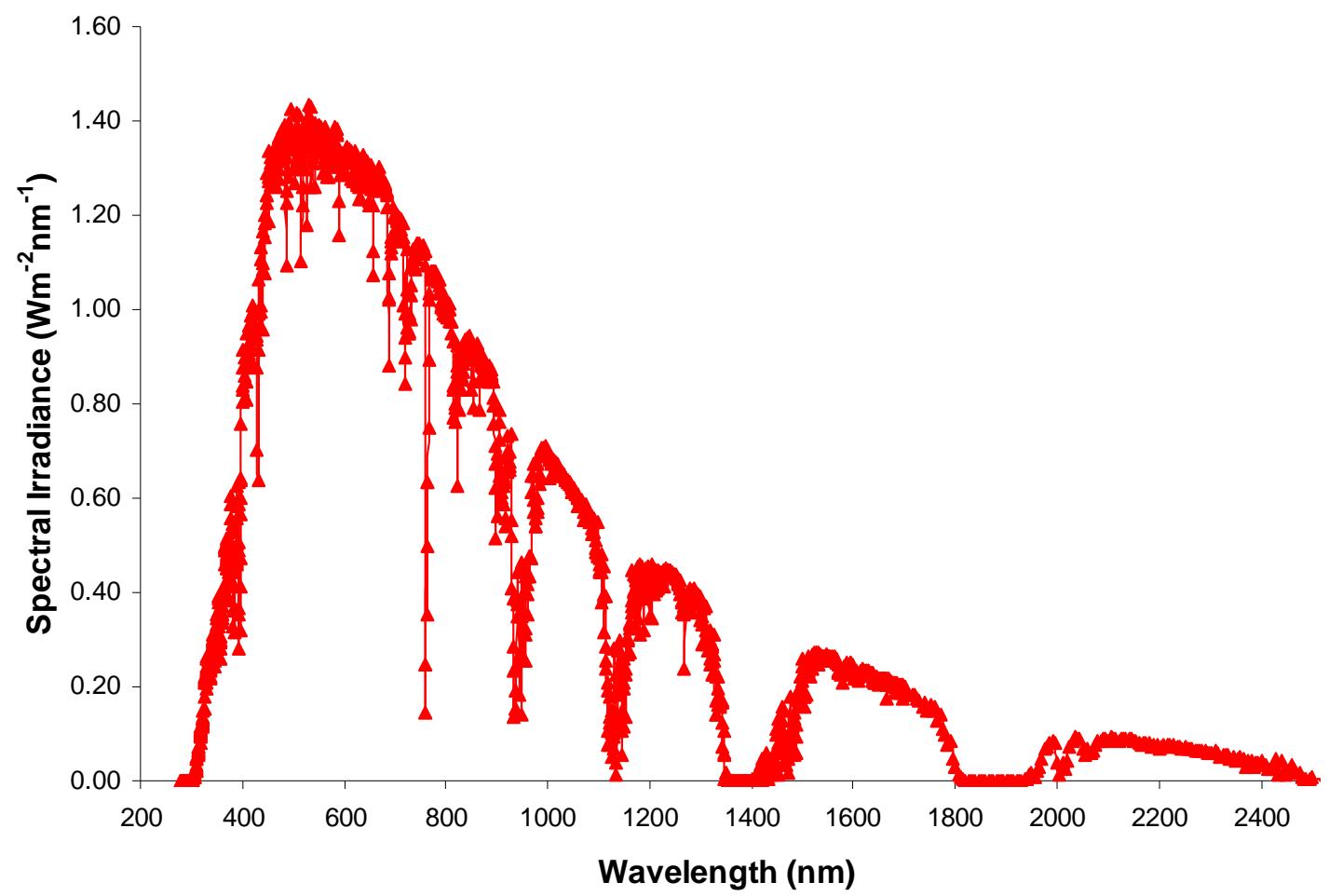

Figure 38. Spectral Irradiance from the Sun. American Society of Testing and Materials (ASTM) G173-03 Reference Spectra Derived from Simple Model of the Atmospheric Radiation Transfer of Sunshine (SMARTS) v. 2.9.2. ${ }^{[29]}$

76 


\section{The Importance of the Silicate Backbone}

The assessment of whether or not the silicate backbone took part in the photochemical process was important for the determination of whether the NCaSil merely provided $\mathrm{Ca}^{2+}$, as indicated in the previous chapter, and the cell process taking place was a thin-layer-electrolyte photogalvanic process, if the silicate was acting was the network material for a soggy sand electrolyte, or as the semiconducting material in a DSSC or photogalvanic cell. To ascertain whether the active component of $\mathrm{NCaSil}$ with iodide/triiodide was $\mathrm{CaI}_{2}$, or the presence of the silicate backbone was important for the cell process, the photoactivity of $\mathrm{CaI}_{2}$ alone was tested and is discussed in this chapter.

The requirement of the silicate backbone was also assessed by comparing the silicate-containing cells to cells containing only iodide/triiodide electrolyte with the equivalent amount of $\mathrm{Ca}^{2+}$ added, observing whether the silicate needed to be in the light path in a beaker cell experiment (Experimental, Part III 9) and assessing whether $\mathrm{CaI}_{2}$-synthetic silicate gels could achieve comparative results.

\section{1 $\quad \mathrm{CaI}_{2}$ Alone}

$\mathrm{CaI}_{2}$ was tested in a gasket cell by itself as a control, with no added electrolyte, the solid quickly becoming wet on absorbing moisture from the atmosphere. The normal gasket cells had a volume of $0.0104 \mathrm{~cm}^{3}$. This meant that there was approximately $1.415 \times 10^{-4} \mathrm{~mol}$ of $\mathrm{Ca}^{2+}$ and $2.830 \times 10^{-4} \mathrm{~mol}$ of $\mathrm{I}^{-} / \mathrm{I}_{3}^{-}\left(\mathrm{CaI}_{2}: \rho=4.0 \mathrm{~g} / \mathrm{cm}^{3}, \mathrm{Mr}=293.9 \mathrm{gmol}^{-1}\right)^{[127]}$ present in the cell. Comparatively, the RNCaSil cells (in a water iodide/triiodide electrolyte) contained around $5.2 \times 10^{-6} \mathrm{~mol} \mathrm{of} \mathrm{Ca}^{2+}$ and $8.233 \times 10^{-3} \mathrm{~mol}$ of $\mathrm{I}^{-} / \mathrm{I}_{3}{ }^{-}$(RNCaSil: $20.9 \% \mathrm{Ca}^{2+}$ ). Thus, the $\mathrm{CaI}_{2}$ cells had more $\mathrm{Ca}^{2+}$ present than $\mathrm{RNCaSil}$ cells, but less iodide/triiodide.

The $\mathrm{CaI}_{2}$ cells functioned with an average $V_{o c}$ of $57.6 \pm 0.9 \mathrm{mV}$ and $I_{s c}$ of $2.9 \pm 0.2 \mu \mathrm{A} / \mathrm{cm}^{2}$ (Figure 39). Comparatively, a water iodide/triiodide electrolyte usually had a lower voltage (varying between 25 to $45 \mathrm{mV}$ ), but a higher current. Even with no $\mathrm{CaCl}_{2}$ added, the iodide/triiodide cells had a current of 8.8 $\pm 0.3 \mu \mathrm{A} / \mathrm{cm}^{2}$, which increased as more $\mathrm{CaCl}_{2}$ was added. This is discussed further below (Part IV 4.3.1). Similarly, whether or not $\mathrm{CaCl}_{2}$ was pre-added to the iodide/triiodide electrolyte, RNCaSilcontaining cells exhibited slightly lower $V_{o c}$ than that of the $\mathrm{CaI}_{2}$ by itself, but higher currents. 


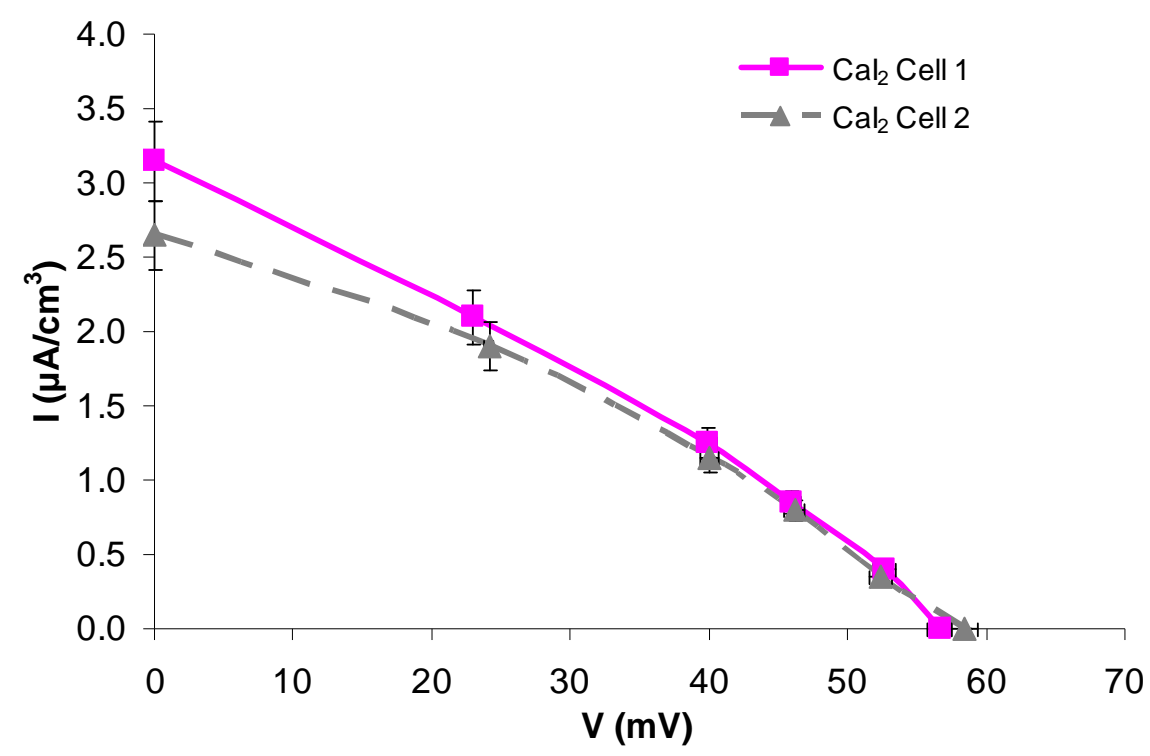

Figure 39. Current versus Voltage of $\mathrm{CaI}_{2}$ in a Gasket Cell.

The relatively low current of the $\mathrm{CaI}_{2}$ cell was attributable to the solid nature of the sample, as it inhibited charge mobility. Presumably, triiodide must have been present in order for there to be a photoactive redox couple, even though no iodine was added to the sample. This was confirmed by UVvis, which exhibited peaks at 351 and $288 \mathrm{~nm}$ (Figure 40), representing triiodide. ${ }^{[60]}$ The lower current obtained for $\mathrm{CaI}_{2}$ could have been indicative of the silicate structure's importance.

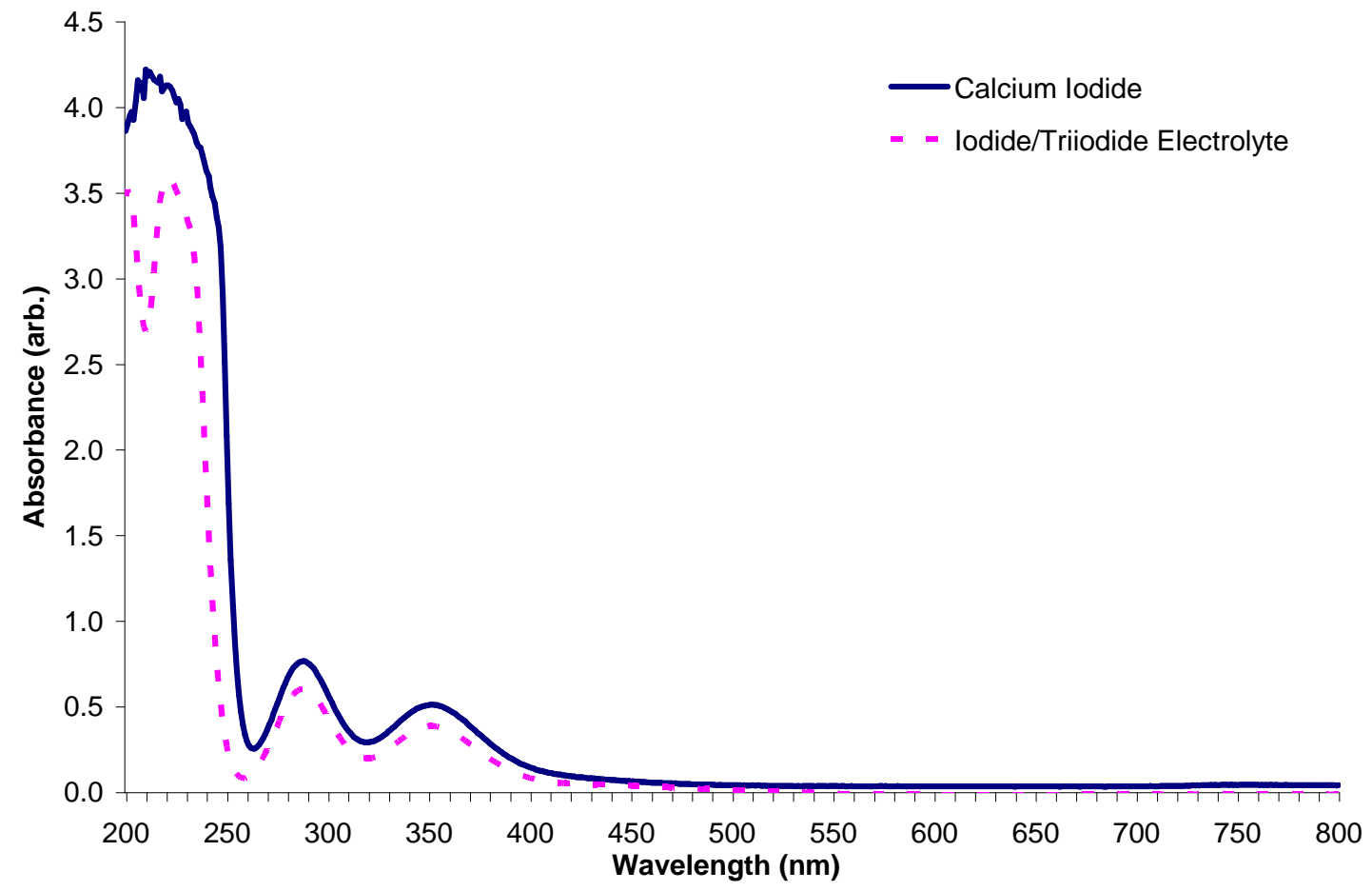

Figure 40. UV-vis of $\mathrm{CaI}_{2}$ in Water Compared to the Iodide/Triiodide Electrolyte. 
Though not decisive (due to the different compositions of $\mathrm{Ca}^{2+}$ and $\mathrm{I}^{-} / \mathrm{I}_{3}^{-}$in the cells), these results indicated that the silicate structure in $\mathrm{NCaSil}$ was indeed important to the cell process and, though $\mathrm{CaI}_{2}$ could function in a cell alone, the silicate backbone contributed significantly to the cell process, allowing for a higher current. The results did not clarify whether the presence of silicate resulted in the formation of a soggy sand electrolyte or whether the silicate was acting as a semiconductor.

\subsection{Electrolyte with Equivalent $\mathrm{Ca}^{2+}$ Additive}

A control cell of just the iodide/triiodide electrolyte was tested, with $\mathrm{CaCl}_{2}$ added to be equivalent to the $\mathrm{Ca}^{2+}$ that would be present from the RNCaSil in an RNCaSil cell, assuming full dissociation (Experimental, Part III 2.2.3). These experiments (utilising beaker cells) showed how integral a part the silicate structure played in the cell process. Where the current and potentials obtained for related RNCaSil cells were approximately $0.4 \mu \mathrm{A} / \mathrm{cm}^{2}$ and $40 \mathrm{mV}$ respectively, those obtained for the $\mathrm{Ca}^{2+}$ equivalent electrolyte cell were only approximately $0.09 \mu \mathrm{A} / \mathrm{cm}^{2}$ and $13 \mathrm{mV}$. Therefore, it was concluded that the RNCaSil was not merely acting as a source of $\mathrm{Ca}^{2+}$.

\subsection{Importance of Silicate in the Light Path}

In order to further determine the importance of the silicate backbone to the cell process, larger cells were set up in beakers with an overhead stirrer. Usually, with the beaker cells, the NCaSil settled to the bottom of the beaker and it was only with constant stirring over time that the silicate eventually dispersed more evenly throughout the electrolyte. Overhead stirring was used and staggered, so that the sample was stirring for 10 minutes, followed by 10 minutes of non-stirring, and so forth.

The current did appear to be affected by whether the silicate was in the light path (Figure 41(b)). The time periods between 20 to 30,40 to 50 and 60 minutes onwards seemed to indicate that stirring increased the current. Whereas, the unstirred periods, between 30 to 40 and 50 to 60 minutes, showed a plateau in the current. However, this data was not absolutely determinative, as other beaker cells also showed an increase in current over time, particularly in the first two hours of illumination (Part IV 8.1). This could be observed in the period between 10 to 20 minutes, where the current continued to climb, free of stirring, and at a rate greater than when stirring occurred. 

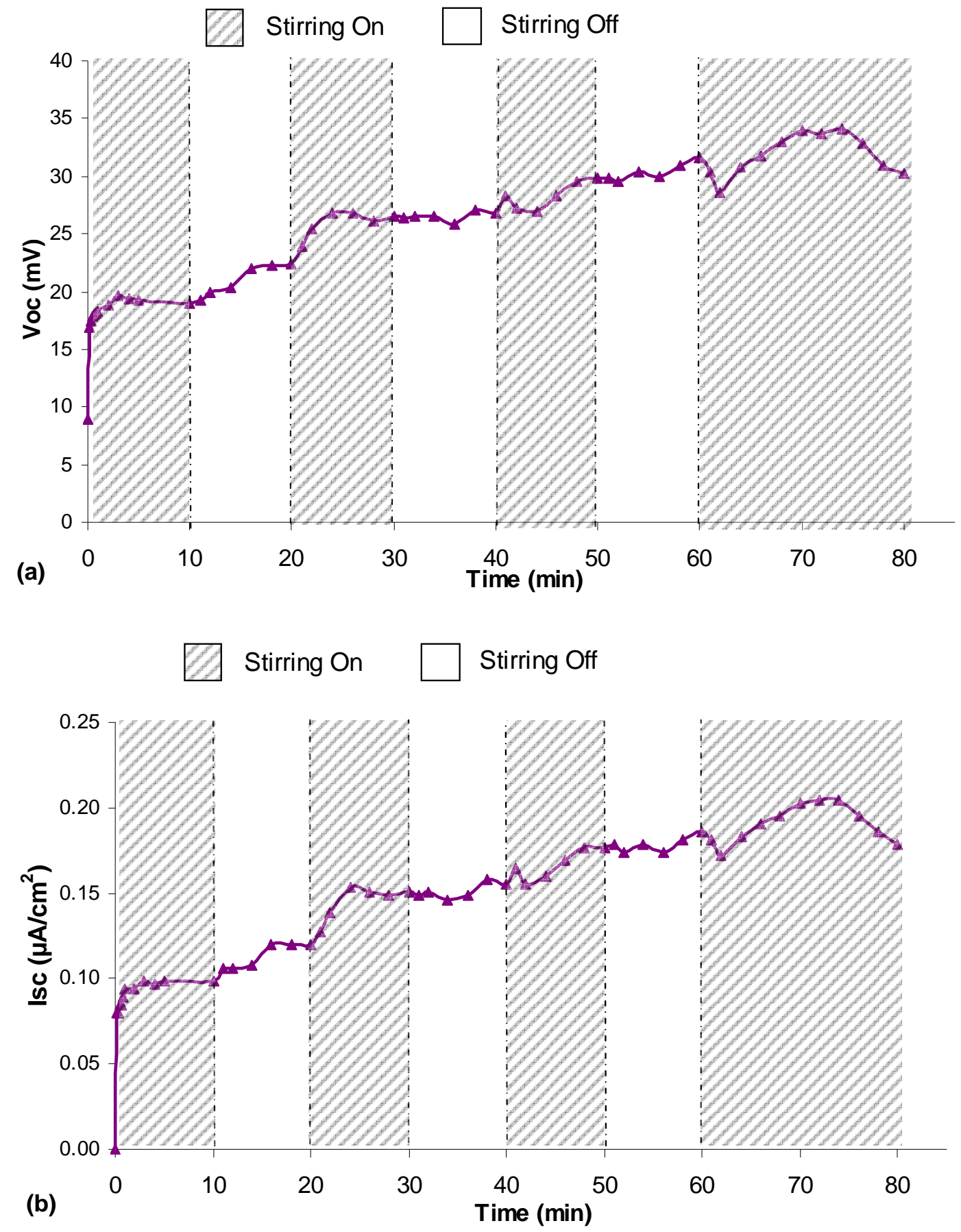

Figure 41. Stirring of RNCaSil with Water Iodide/Triiodide Electrolyte in a Beaker Cell; (a) $V_{o c}$ and (b) $\mathbf{I}_{s c}$.

It appeared that the $V_{o c}$ was indifferent to whether or not the silicate was directly in the light path (Figure 41(a)). Generally, it increased over time, whether or not the magnetic stirrer was on. This upward trend was observed with other beaker cells over the initial few hours of testing (for example, Part IV 5.2.3). This indicated that the iodide/triiodide's photogalvanic process dominated the cell. 
Because the current and voltage were never stagnant over longer periods of time in these beaker cells, it was impossible to determine if the observed effects were real, or just part of the general upwards trend and the increases and plateaus a coincidence. Therefore, this experiment only tentatively indicated that the silicate structure did take part in the photocell process, though it was likely dominated by the iodide/triiodide couple.

\subsection{Synthetic $\mathrm{CaI}_{2}$ Silicate Gels}

Gels were prepared in order to determine whether synthetic silicates were able to exhibit similar properties to a gel NCaSil (discussed in depth in Part IV 8 and 9). Gels made from sodium silicate, $\mathrm{CaI}_{2}$ and, in some gels, other metal sources, proved to be non-photoactive. Some of the gels showed low activity, but this was likely due to the small amount of $\mathrm{CaI}_{2}$ present, which was supported by the fact that a blank gel, free of any $\mathrm{CaI}_{2}$ showed no activity. Synthetic $\mathrm{Ca}^{2+}-\mathrm{TEOS}$ gels also proved nonphotoactive.

The failure of these gels to show any significant photoactivity suggested that the specific backbone of NCaSil was indeed supportive of the photochemical process taking place. This further indicated that

the NCaSils were not simply a source of $\mathrm{Ca}^{2+}$ to aid in charge transport of the electrolyte's photochemical process.

\subsection{Conclusions}

The experiments discussed in this chapter indicated that the silicate backbone of NCaSils played an integral part in the increased cell performance upon the addition of NCaSil to an iodide/triiodide electrolyte. The improvement was not solely due to $\mathrm{Ca}^{2+}$ release from NCaSil. 


\section{The Electrolyte}

\subsection{Different NCaSils:Iodide/Triiodide Electrolyte Ratios}

Different ratios of RNCaSil to the electrolyte were tested. This was carried out in normal gasket cells, with the iodide/triiodide electrolyte. It was expected that this would elucidate a peak-performance ratio, which could then be used for later experiments. A large variance in potential was not anticipated, due to results discussed in chapter 1.1.1, which showed that the addition of NCaSil to the iodide/triiodide electrolyte only raised the $V_{\text {oc }}$ slightly. Greater consequences on the cell current were expected, because adding NCaSil affected the cell's $\mathrm{Ca}^{2+}$ content, changed the viscosity of the electrolyte, and earlier studies had shown that the silicate backbone contributed to higher $I_{\mathrm{sc}}$ values.

No significant change in $V_{\mathrm{oc}}$ was observed until the proportion of RNCaSil to electrolyte solution was greater than or equal to $0.05 \mathrm{~g} / \mathrm{cm}^{3}$ (Figure $42(\mathrm{a})$ ). The iodide/triiodide photogalvanic processes underlying the cell completely dominated its behaviour below this value. The NCaSil had a larger $V_{\mathrm{oc}}$ than the iodide/triiodide electrolyte because NCaSil has a larger band gap of $3.6 \mathrm{eV}$ than that of the major absorption peak of the photogalvanic light-absorbing species of the electrolyte, ${ }^{[60]}$ the triiodide, ${ }^{[106]}$ at $3.44 \mathrm{eV}$. That the potential of the electrolyte was never greatly affected by the addition of NCaSil indicated that the samples were acting as soggy sand electrolytes, where the photoactivity of NCaSil never dominated.

The trend for the $I_{\mathrm{sc}}$ (Figure 42(b)) on increasing the proportion of reinforced NCaSil was clearer than that for the $V_{\mathrm{oc}}$. The $I_{\mathrm{sc}}$ of the electrolyte solution alone was already relatively high, and addition of a small amount of reinforced NCaSil seemed to disturb the electrolyte's photogalvanic process, likely suppressing the iodide/triiodide transitions, indicating that the iodide/triiodide process dominated. It was not until a loading of $0.10 \mathrm{~g} / \mathrm{cm}^{3} \mathrm{RNCaSil}$ was reached that a positive effect of the silicate material was observed. At that concentration, the RNCaSil's acted synergistically with the electrolyte, as in a soggy sand electrolyte.

This hypothesis was substantiated by UV-vis. UV-vis of the water iodide/triiodide electrolyte alone and with RNCaSil showed that there was little change in light absorption (above, Figure 28). Thus, the UVvis absorption was dominated by the electrolyte. This supported the theory that when NCaSil was used in conjunction with the iodide/triiodide electrolyte, the NCaSil's photoactivity was either not expressed 
or only present to a minor degree and, instead, the mixture acted as a soggy sand electrolyte. The UVvis was not determinative, as there were two alternative explanations as to why the UV-vis showed the electrolyte dominating absorbance: (1) the ratio of electrolyte to RNCaSil strongly favoured the former; and (2) the RNCaSil tended to settle to the bottom of the test cuvette. However, data on cell performance upon the addition of RNCaSil to the iodide/triiodide electrolyte (Part IV 1.1.1), supported the indication from the UV-vis that light uptake was dominated by the electrolyte solution.
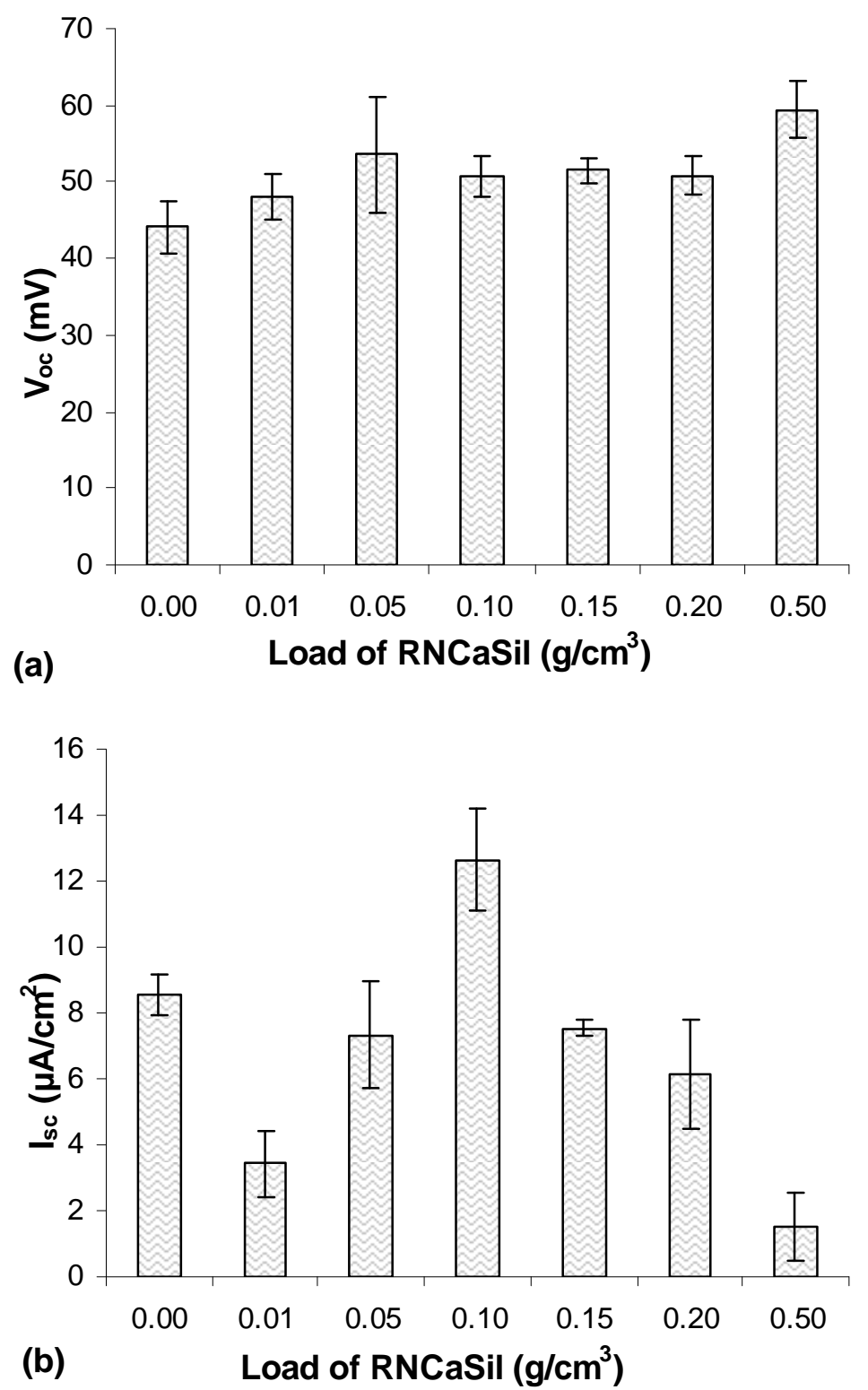

Figure 42. Varying RNCaSil Load in Water Iodide/Triiodide Electrolyte with $5 \mathrm{wt} \% \mathrm{CaCl}_{2}$; (a) $V_{o c}$ and (b) $I_{s c}$. 
As the loading of RNCaSil increased beyond $0.10 \mathrm{~g} / \mathrm{cm}^{3}$, there was a drop in the $I_{\mathrm{sc}}$. This was attributed to RNCaSil blocking transport processes in the cell. The redox couple became sorbed in the RNCaSil and ineffective. The maximum $I_{\mathrm{sc}}$ was obtained with a loading of $0.10 \mathrm{~g} / \mathrm{cm}^{3} \mathrm{RNCaSil}$; most subsequent tests were performed at this loading.

\subsection{Effect of Electrolyte Additives}

\subsection{1 $\mathrm{CaCl}_{2}$ Additive}

Different amounts of $\mathrm{CaCl}_{2}$ (by wt\%) were added to the electrolyte solution and tested in a cell by themselves and with RNCaSil. This was to find the ideal $\mathrm{CaCl}_{2}$-additive content. As expected, the potential was greater in the RNCaSil cells than cells of electrolyte solutions alone (Figure 43(a)). When the electrolyte was tested alone, the $V_{o c}$ generally increased relative to the $\mathrm{Ca}^{2+}$ content. This was consistent with literature reports of DSSCs, ${ }^{[68]}$ where the addition of $\mathrm{Ca}^{2+}$ (a relatively large cation) would shift the conduction band of the semiconducting material slightly more negative, increasing the $V_{o c}$. This was not the case for the RNCaSil cells, and this was attributed to the fact that the RNCaSil already had a sufficient supply of accessible $\mathrm{Ca}^{2+}$. The result obtained from the pure iodide/triiodide electrolyte was much lower than expected (compared to the literature $)^{[106]}$ and was considered a spike.

The current rose as more $\mathrm{Ca}^{2+}$ was added for both the electrolyte alone and the RNCaSil-containing cells (Figure 43(b)). This was expected, given that the addition of $\mathrm{Ca}^{2+}$ should have increased the number of charge carriers present. It was suspected that if the load of $\mathrm{CaCl}_{2}$ were increased much further a decline in current would be observed, due to charge impediments caused by increased viscosity obstructing the ions. However, this could not be tested as $25 \mathrm{wt} \%$ was the maximum amount of $\mathrm{CaCl}_{2}$ that could be made to dissolve in the electrolyte solution.

The only sample where the RNCaSil was clearly more efficient than the electrolyte alone was the 5 wt $\% \mathrm{CaCl}_{2}$ electrolyte sample. Though these powder cells exhibited greater potentials than the electrolyte alone (due to the larger band gap of NCaSil than iodide/triiodide), currents of the RNCaSilcontaining cells were generally lower. It was believed that the reason for this was due to the solubility of $\mathrm{Ca}^{2+}$. At low levels of $\mathrm{CaCl}_{2}$, there was insufficient $\mathrm{Ca}^{2+}$ for the RNCaSil to work synergistically with the electrolyte and the addition of RNCaSil merely disturbed the photogalvanic process of iodide/triiodide, by inhibiting the iodide/triiodide transitions (Part IV 1.1.5). It was not until 5 wt\% $\mathrm{CaCl}_{2}$ in water was reached that a positive combined effect was observed. 

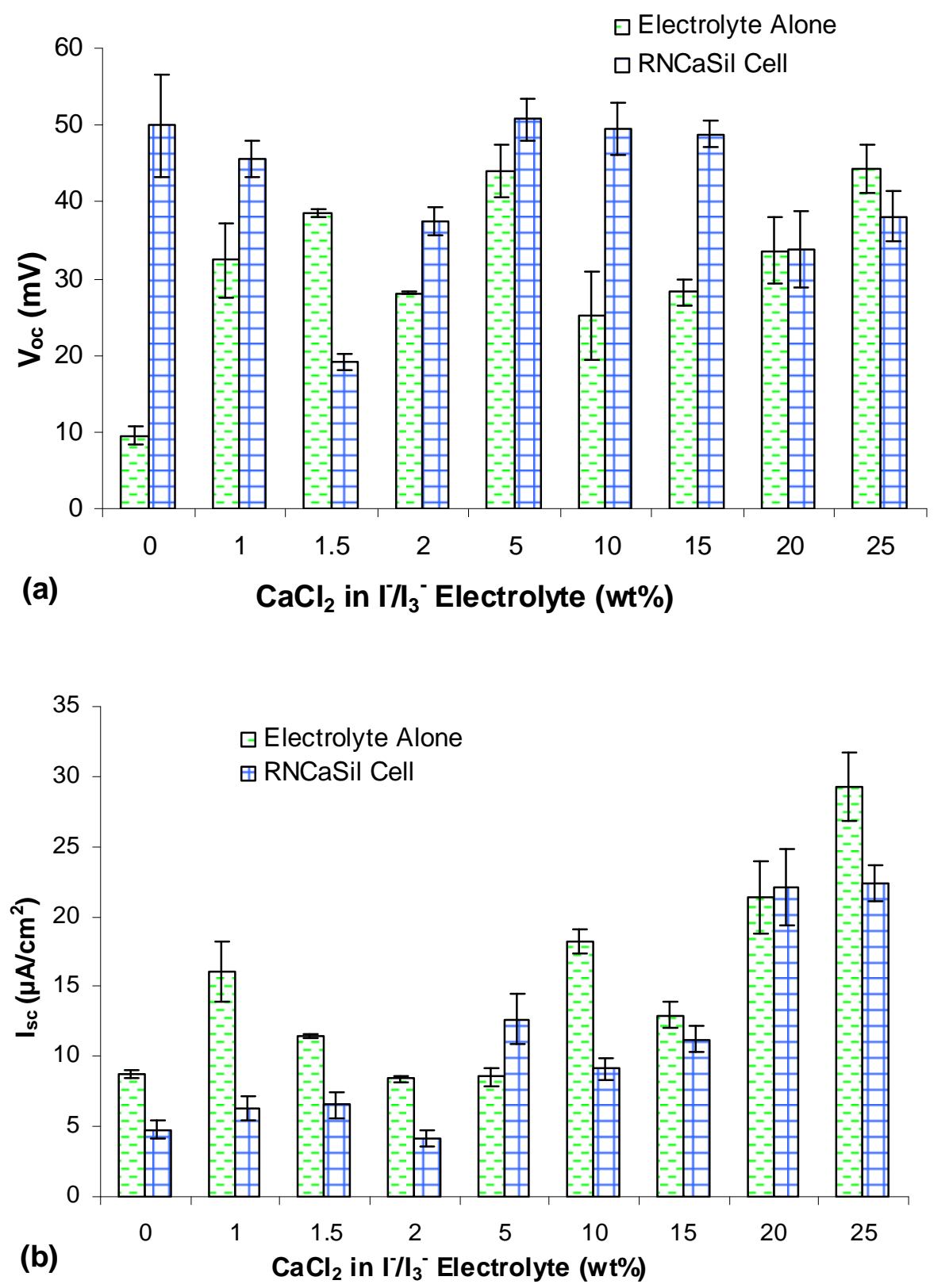

Figure 43. The Effect of $\mathrm{CaCl}_{2}$ content in the Iodide/Triiodide Electrolyte (100\% Water); (a) $V_{o c}$ and (b) $I_{s c}$.

As the concentration of $\mathrm{CaCl}_{2}$ increased further, both the currents of the electrolyte solution alone and with RNCaSil continued to climb, but those of the RNCaSil containing cells were lower than the $I_{s c}$ of their related electrolyte cells. This was attributed to the precipitation of $\mathrm{Ca}(\mathrm{OH})_{2}$ on the addition of the alkaline RNCaSil, when the concentration of $\mathrm{CaCl}_{2}$ became too high, which removed $\mathrm{Ca}^{2+}$ from the electrolyte. $\mathrm{CaCl}_{2}$ is very soluble in water alone $\left(\mathrm{K}_{\mathrm{sp}}=1672 \mathrm{~mol} / \mathrm{dm}^{3}\right.$, calculated). However, $\mathrm{Ca}(\mathrm{OH})_{2}$ is not $\left(\mathrm{K}_{\mathrm{sp}}=6.4 \times 10^{-6} \mathrm{~mol} / \mathrm{dm}^{3}\right)^{[127]}$ and will precipitate at high $\mathrm{pH}$ values. The calculated solubility of $\mathrm{Ca}^{2+}$, depending on the $\mathrm{pH}$ value (or $\left[\mathrm{OH}^{-}\right]$), can be seen in Figure 44. 


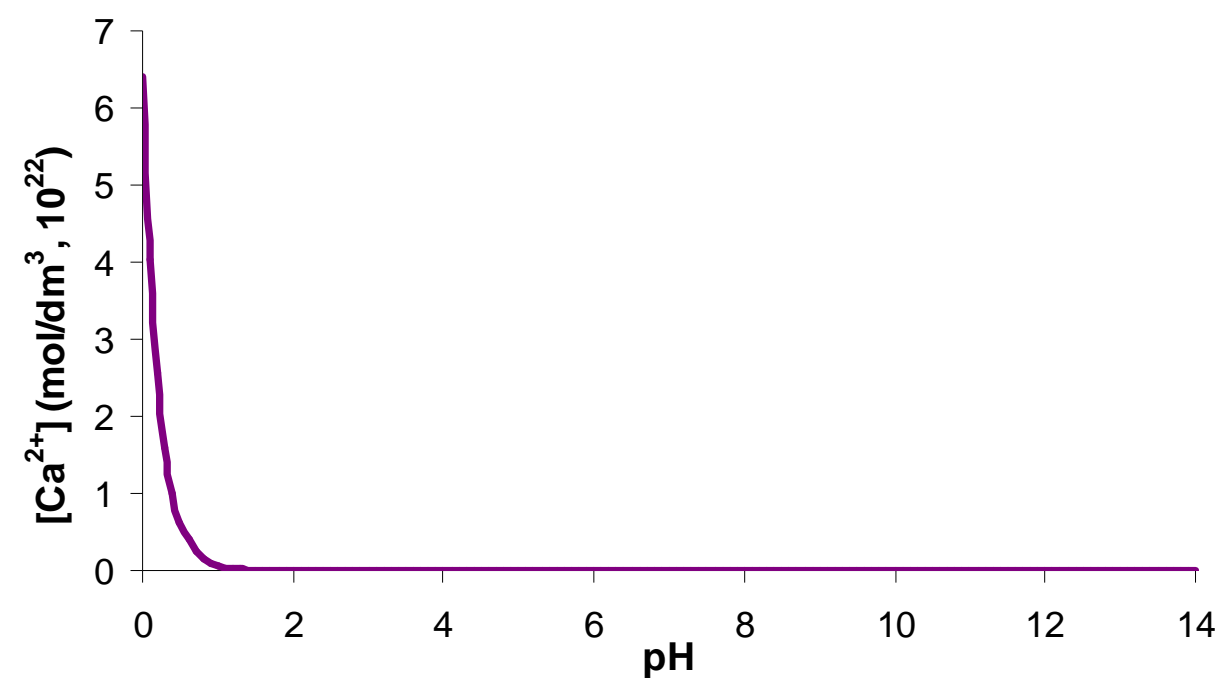

Figure 44. $\mathrm{Ca}^{2+}$ Solubility at Different pHs.

At a $\mathrm{pH}$ of 11.5 , the solubility of $\mathrm{Ca}^{2+}$ is approximately $0.64 \mathrm{~mol} / \mathrm{dm}^{3}$ (see Appendix, Part VII 7, Table 9). At 5 and $10 \mathrm{wt} \%$, the concentration of $\mathrm{CaCl}_{2}$ was $0.527 \mathrm{~mol} / \mathrm{dm}^{3}$ and $1.14 \mathrm{~mol} / \mathrm{dm}^{3}$, respectively. Thus, with the $\mathrm{CaCl}_{2}$ contents tested that were higher than $5 \mathrm{wt} \%, \mathrm{Ca}(\mathrm{OH})_{2}$ would have precipitated.

\subsubsection{Alternative Additives to $\mathrm{Ca}^{2+}$}

In order to determine whether the results obtained by using $5 \mathrm{wt} \% \mathrm{CaCl}_{2}$ in the iodide/triiodide electrolyte, both with and without RNCaSil, were specific to the $\mathrm{Ca}^{2+}$, iodide/triiodide electrolytes were prepared with $\mathrm{SrCl}_{2}$ and $\mathrm{MgCl}_{2}$, equimolar to the $\mathrm{Ca}^{2+}$ in $5 \mathrm{wt} \% \mathrm{CaCl}_{2}$ electrolyte solutions. These were tested both alone and with RNCaSil.

The $\mathrm{Sr}^{2+}$ electrolyte was effective alone (Figure 45); its current almost three times as great as that of the equivalent $\mathrm{Ca}^{2+}$ cell. However, it did not perform well when used in conjunction with RNCaSil. This was the same with the $\mathrm{Mg}^{2+}$. The $\mathrm{Sr}^{2+}$ and $\mathrm{Mg}^{2+}$ cells, with and without RNCaSil, were almost identical; certainly, within experimental error. The degree of similarity, with regards to the attributes of the $\mathrm{Sr}^{2+}$ and $\mathrm{Mg}^{2+}$ cells, was not expected. Given that $\mathrm{Mg}^{2+}$ has an ionic radius of $72 \mathrm{pm},{ }^{[127]}$ a closer resemblance between the $\mathrm{Ca}^{2+}$ and $\mathrm{Sr}^{2+}$ cells was expected. Due to the similarities between $\mathrm{Ca}^{2+}$ and $\mathrm{Sr}^{2+}$ (both are divalent and have similar ionic radii of 100 and $118 \mathrm{pm}$, respectively) ${ }^{[127]}$ it was surprising that the $\mathrm{Sr}^{2+}$ infused electrolyte acted so differently, whether alone or with RNCaSil. 

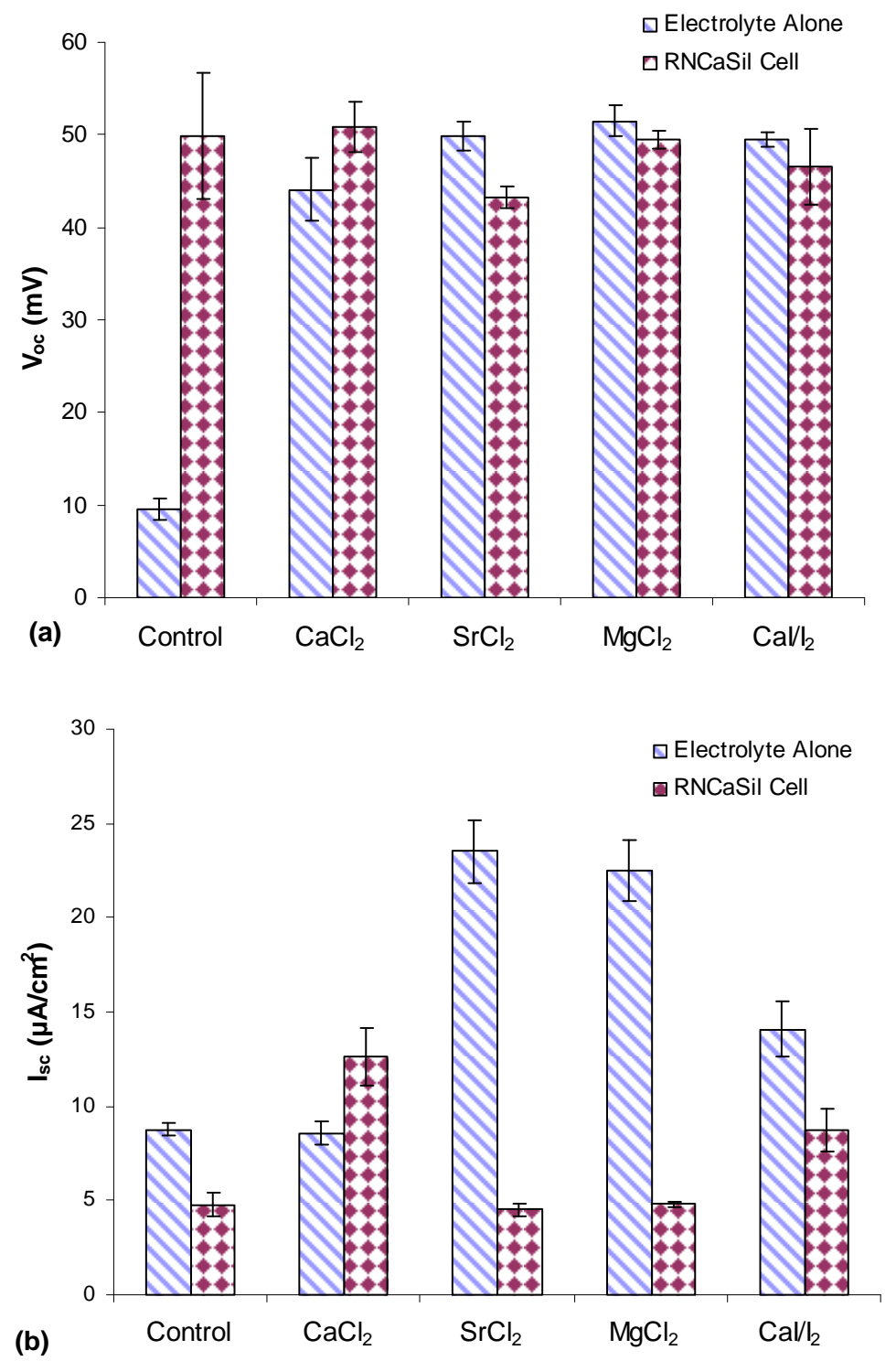

Figure 45. Iodide/Triiodide Electrolyte with Different Additives: $5 \mathrm{wt} \% \mathbf{C a C l}_{2}$ and, Relative to the $\mathbf{C a C l}_{\mathbf{2}} \mathrm{Electrolyte}$, Equimolar Amounts of $\mathrm{SrCl}_{2}$ and $\mathrm{MgCl}_{2}$, and $55.7 \mathrm{~mol} \% \mathrm{Ca}^{2+}$; (a) $V_{o c}$ and (b) $I_{s c}$.

Why the $\mathrm{Sr}^{2+}$ and $\mathrm{Mg}^{2+}$ electrolyte cells behaved so differently from the $\mathrm{Ca}^{2+}$ electrolyte was not resolved. The addition of $5 \mathrm{wt} \% \mathrm{CaCl}_{2}$ to the iodide/triiodide did not significantly affect the current of the electrolyte alone. However, when $\mathrm{Sr}^{2+}$ and $\mathrm{Mg}^{2+}$ were used instead, the current was almost tripled. Interestingly, the $5 \mathrm{wt} \% \mathrm{CaCl}_{2}$ solution was the only electrolyte where the current was altered positively on the addition of RNCaSil. With the pure iodide/triiodide, the current was adversely affected; the RNCaSil appeared to inhibit the photogalvanic process of the electrolyte alone. The current for the $\mathrm{Sr}^{2+}$ and $\mathrm{Mg}^{2+}$ cells were similarly suppressed on the addition of RNCaSil. That the RNCaSil interacted well with a $\mathrm{Ca}^{2+}$ electrolyte (compared to the other two electrolytes) was not surprising, given the obvious affinity of RNCaSil for calcium. 
The results could not be explained by the effect of cations that has been observed for DSSCs (Part I 1.4.1.2), for which it has been found that large cations decrease the current, but have a positive effect on the potential, and conversely for smaller cations. The effect is caused by the cations' interaction with the $\mathrm{TiO}_{2}$ surface, thus one would not expect to see the trend with the electrolytes alone, but only with the RNCaSil containing cells, if they have similar behaviour to $\mathrm{TiO}_{2}$ DSSCs. If the effect were the same for the RNCaSil cells, one would expect the $\mathrm{Mg}^{2+}$ infused cells to have larger currents and smaller potentials and, moving to $\mathrm{Ca}^{2+}$ then $\mathrm{Sr}^{2+}$ cells, the voltage would grow as the $I_{s c}$ declined. That this was not seen was indicative of the fact that any cations present did not interact with the RNCaSil and affect its interfacial energy, in the same way as observed with the $\mathrm{TiO}_{2}$ surface.

Though different surface interactions with cationic species between $\mathrm{TiO}_{2}$ and $\mathrm{RNCaSil}$ were expected, given their different structures, one would have anticipated some interaction of RNCaSil (and indeed all NCaSils) with different cations. This was because of RNCaSil's large and predominantly anionic surface, being covered with - $\mathrm{OH}$ groups. Thus, the interaction between cations and RNCaSil did not have the same impact on the materials potential and driving force of injection efficiency (or current) as has been shown with $\mathrm{TiO}_{2}$. This further indicated that the RNCaSil simply did not act in the same manner as $\mathrm{TiO}_{2}$ and its cells not like DSSCs, but more like soggy sand electrolytes.

It can be noted from the previous discussion (Part IV 4.1) that, whether the RNCaSil interacted with the $\mathrm{Ca}^{2+}$ electrolyte in a cell enhancing fashion, was greatly affected by the electrolyte to RNCasil ratio used. It was possible (and indeed likely) that the RNCaSil $\mathrm{Sr}^{2+}$ and $\mathrm{Mg}^{2+}$ electrolyte cells exhibited poor currents relative to the electrolytes alone, because the optimum ratios were not utilised. It was suspected that the ratio used for this experiment was such that the RNCaSil merely hindered the iodide/triiodide's photogalvanic process, rather than working in conjunction with it.

\subsection{3 $\mathrm{Ca}^{2+}$ Only Added}

To determine the impact that the presence of the $\mathrm{Cl}^{-}$anion (from the $\mathrm{CaCl}_{2}$ additive) had on cell performance, an electrolyte was prepared where only $\mathrm{Ca}^{2+}$ was present and not $\mathrm{Cl}^{-}$. This was achieved by maintaining the same ratios of water, iodine and iodide normally used in the electrolyte, but with the iodide content being supplied by $\mathrm{CaI}_{2}$. The $\mathrm{Ca}^{2+}$ content in this electrolyte was $55.7 \mathrm{~mol} \%$ of that in the $5 \mathrm{wt} \% \mathrm{CaCl}_{2}$ iodide/triiodide electrolyte. 
Interestingly, the currents obtained from the electrolyte alone were higher than those obtained by the 5 wt $\% \mathrm{CaCl}_{2}$ electrolyte, even though the latter had almost twice as much $\mathrm{Ca}^{2+}$ (above, Figure 45). This indicated that the $\mathrm{Cl}^{-}$ion did not enhance the process, but in fact inhibited it, by not partaking in any photo-process, not carrying any charge and impeding the movement of positive charges. Hence, the $\mathrm{Ca}^{2+}$ was clearly the active component of the $\mathrm{CaCl}_{2}$. Such a finding supported a photogalvanic rather than DSSC system, as in the former the electrolyte transports electrons, whereas in the latter, the electrolyte is the hole conductor. This did not elucidate whether semiconductor, thin-layer-electrolyte or soggy sand electrolyte cells were made. In semiconductor photogalvanic cells, the electrolyte transports electrons rather than holes and in the photoactive process of the iodide/triiodide only negatively charged species are present, either of which would be enhanced by the presence of cations. When used in conjunction with RNCaSil, the current was relatively lower, which was attributed to a mismatch of the ratio used.

\subsubsection{Tetrabutylammonium Hexafluorophosphate $\left(\mathrm{PF}_{6}{ }^{+} \mathrm{N}\left(\mathrm{C}_{4} \mathrm{H}_{9}\right)_{4}\right)$}

Having as non-liquid an electrolyte as possible is deemed to be desirable, due to the stability usually associated with more solid, non-evaporable electrolytes. Hence, tetrabutylammonium hexafluorophosphate was tested as an additive to the iodide/triiodide electrolyte in the hopes of lowering the solvent requirement. The electrolyte was tested with RNCaSil (Experimental, Part III 2.2.5).

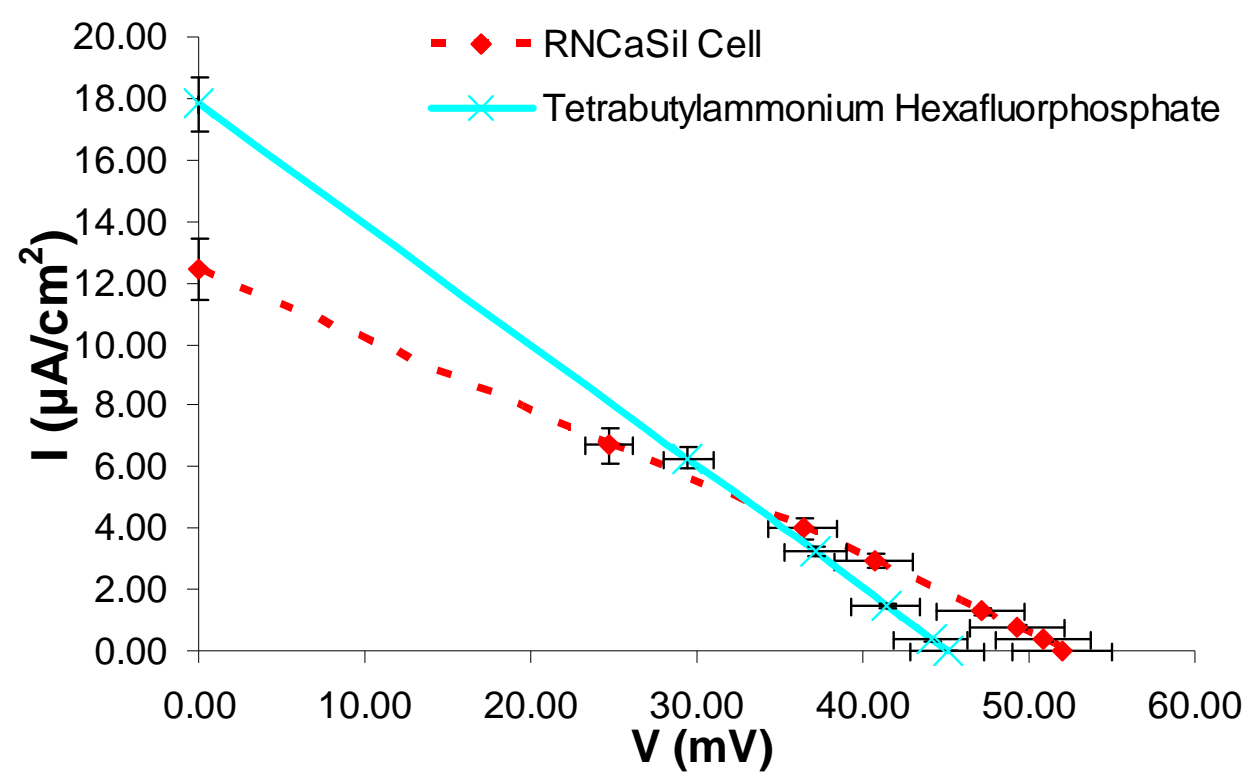

Figure 46. RNCaSil with Tetrabutylammonium Hexafluorophosphate Compared to RNCaSil in Water Based Iodide/Triiodide Electrolyte (5 $\left.\mathrm{wt} \% \mathrm{CaCl}_{2}\right)$. 
An $I_{s c}$ of $17.8 \pm 0.9 \mu \mathrm{A} / \mathrm{cm}^{2}$ and $V_{o c}$ of $45.1 \pm 2.3 \mathrm{mV}$ was achieved (Figure 46). The current was greater than that from RNCaSil used in conjunction with the iodide/triiodide with $5 \mathrm{wt} \% \mathrm{CaCl}_{2}$ and the potential was comparable. However, the sample still required a small amount of water to solvate the iodine and KI. Therefore, although the presence of the ionic tetrabutylammonium hexafluorophosphate increased cell current, the sample was not completely solid.

\subsection{The Solvent Effect}

\subsubsection{Different Solvents - Gasket Tests}

Different solvents were used to solvate the iodide/triiodide. These were made up with $70 \mathrm{wt} \%$ of an organic solvent and $30 \mathrm{wt} \%$ water, the latter being required to get sufficient solvation. The iodide and triiodide concentrations remained unchanged. It should be noted that the conentration of the organic solvent differered, as weight percentages were used. However, there was neglible ionic activity in the organic solvent and the water concentration was kept constant. The different solvents tested were: ethanol, acetone, acetonitrile, 2-ethoxyethanol, ethylene glycol, propan-1-ol, propan-2-ol and tetrahydrofuran (THF). The different electrolyte solutions were tested in normal gasket cells, both alone and with RNCaSil. The data obtained has been plotted with respect to increasing viscosity of the electrolyte solutions and increasing dielectric constants of the solvents in the organic electrolytes (Table 8).

Table 8. Different Viscosities and Dielectric Constants of Electrolyte Solutions Tested.

\begin{tabular}{|c|c|c|}
\hline Iodide/Triiodide Electrolyte Solutions & $\begin{array}{c}\text { Viscosity } \\
(\mathrm{mPaS}) \\
\left(\text { at } 21.9^{\circ} \mathrm{C}\right) \\
\end{array}$ & $\begin{array}{c}\text { Dielectric Constant of } \\
\text { the Organic } \\
\text { Solvents }{ }^{*[130]} \\
\end{array}$ \\
\hline $100 \%$ Water $\left(\mathrm{No} \mathrm{CaCl}_{2}\right)$ & 1.10 & 80.10 \\
\hline $100 \%$ Water $\left(5 \mathrm{wt} \% \mathrm{CaCl}_{2}\right)$ & 1.48 & 80.10 \\
\hline $70 \mathrm{wt} \%$ Ethanol $30 \mathrm{wt} \%$ Water & 2.68 & 25.30 \\
\hline $70 \mathrm{wt} \%$ Acetone $30 \mathrm{wt} \%$ Water & 1.83 & 21.01 \\
\hline $70 \mathrm{wt} \%$ Acetonitrile $30 \mathrm{wt} \%$ Water & 2.04 & 36.64 \\
\hline $70 \mathrm{wt} \%$ 2-Ethoxyethanol $30 \mathrm{wt} \%$ Water & 2.29 & $13.38^{\dagger}$ \\
\hline $70 \mathrm{wt} \%$ Ethylene Glycol $30 \mathrm{wt} \%$ Water & 2.93 & 41.40 \\
\hline 70 wt $\%$ Propan-1-ol 30 wt $\%$ Water & 2.92 & 20.80 \\
\hline 70 wt $\%$ Propan-2-ol 30 wt $\%$ Water & 2.61 & 20.18 \\
\hline $70 \mathrm{wt} \%$ Tetrahydrofuran $30 \mathrm{wt} \%$ Water & 2.84 & 7.52 \\
\hline
\end{tabular}


The dielectric constant (or permittivity) of the solvents is the ratio of the electric displacement $D$ to the electric field strength $E$ when an external field is applied. ${ }^{[130]}$ The values used here are the relative permittivities, which is the ratio of the actual permittivity to the permittivity of a vacuum. They are dimensionless. ${ }^{[130]}$

There was no obvious trend in cell performance relative to electrolyte viscosity or dielectric constant (Figures 47 and 48). This indicated that cell performance was not dominated by physical properties of the electrolyte solvent. The physical properties of the electrolyte solvent would have had some affect, which resulted in micro-trends between a few data points. But, as there was no overall trend, cell performance must also have been dependant on the chemical relationship between the RNCaSil and the electrolytes, i.e., van der Vaals interactions. That this was indeed the case was supported by the fact that the current only increased upon the addition of RNCaSil to the electrolyte with some solvents, namely ethanol, ethoxyethanol and THF. This inferred that a dominating factor was the RNCaSil/solvent interaction and the effect of the solvent on the RNCaSil-iodine interaction, supporting the soggy sand electrolyte postulation.

It was expected that the current would decrease as viscosity increased, as is usually the case. But, here, the electrolytes alone and RNCaSil cells seemed indifferent to their viscosity. That the viscosity appeared to have no pattern of effect indicated that the cells were not diffusion controlled, the way DSSCs are. A conclusion of non-diffusion control was supported by investigations into the effect of cell depth (discussed below, Part IV 5.2).

With respect to the electrolyte alone, cell performance was, at least to some degree, dependent on the donor number $(\mathrm{DN})$ of the solvent, as observed by Kebede et al. ${ }^{[60]}$ They reported a donor-acceptor type of interaction, in which the solvent was the Lewis base while iodine was the Lewis acid, in other words, an iodine-solvent complex formed. The increase of Lewis basicity of solvents (i.e., the increase in DN) strenghthened the interaction between solvents and iodine. It was observed that this resulted in a larger $V_{o c}$ and smaller $I_{s c}$. This trend could be observed from some of the results obtained in this experiment. For example: acetonitrile and ethanol have DNs of 14.1 and 20, respectively. ${ }^{[60,131]}$ Thus, one would have expected the trend of $V_{o c}$ to be acetonitrile < ethanol; and for the $I_{s c}$ to have been ethanol < acetonitrile, which was observed. 

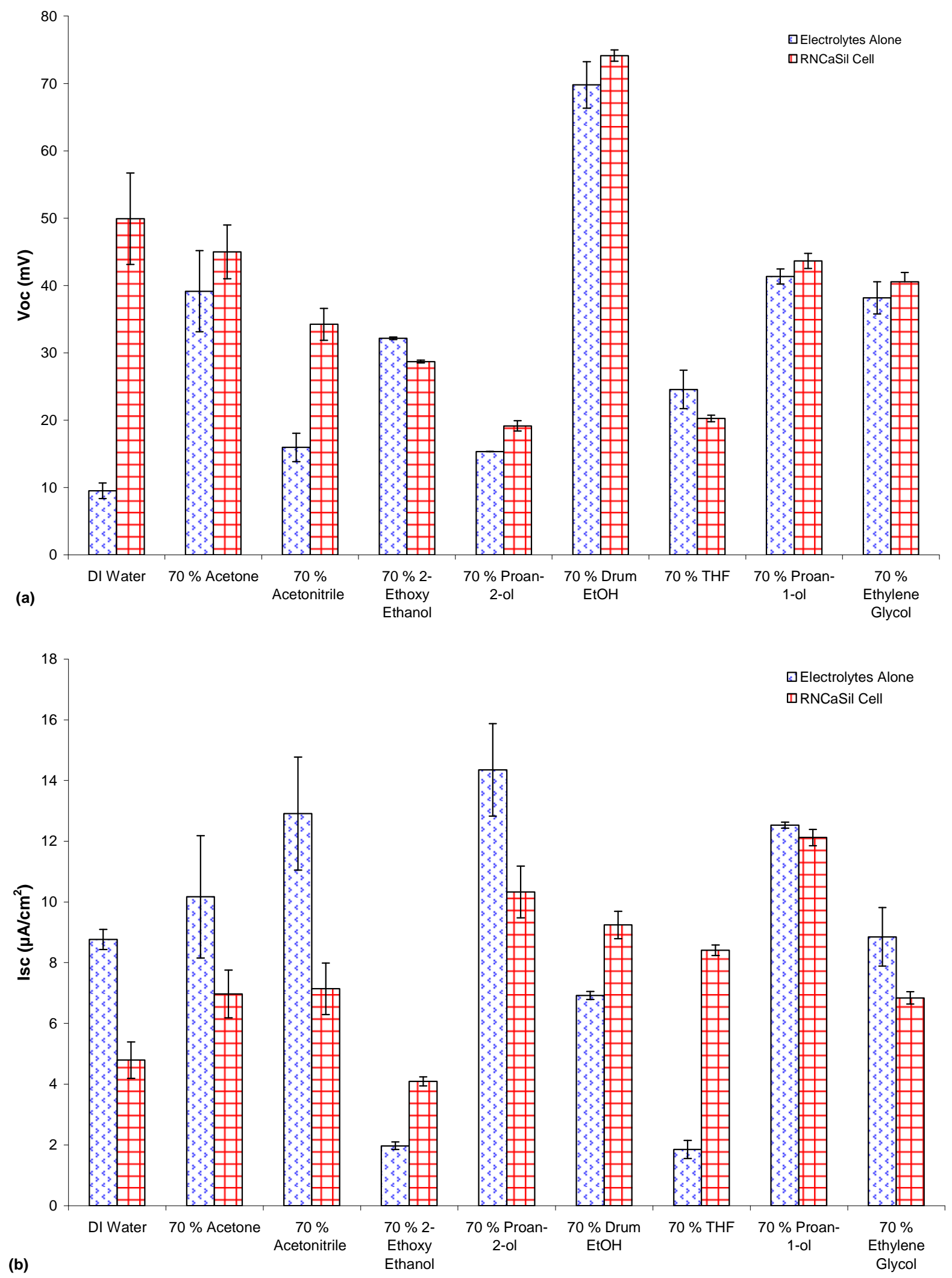

Figure 47. The Effect of Iodide/Triiodide Electrolyte Solvent - Plotted in Order of Increasing Viscosity of the Electrolyte Solution: (a) $V_{o c}$ and (b) $I_{s c}$. The Secondary Solvent $(30$ wt\%) was Water. 

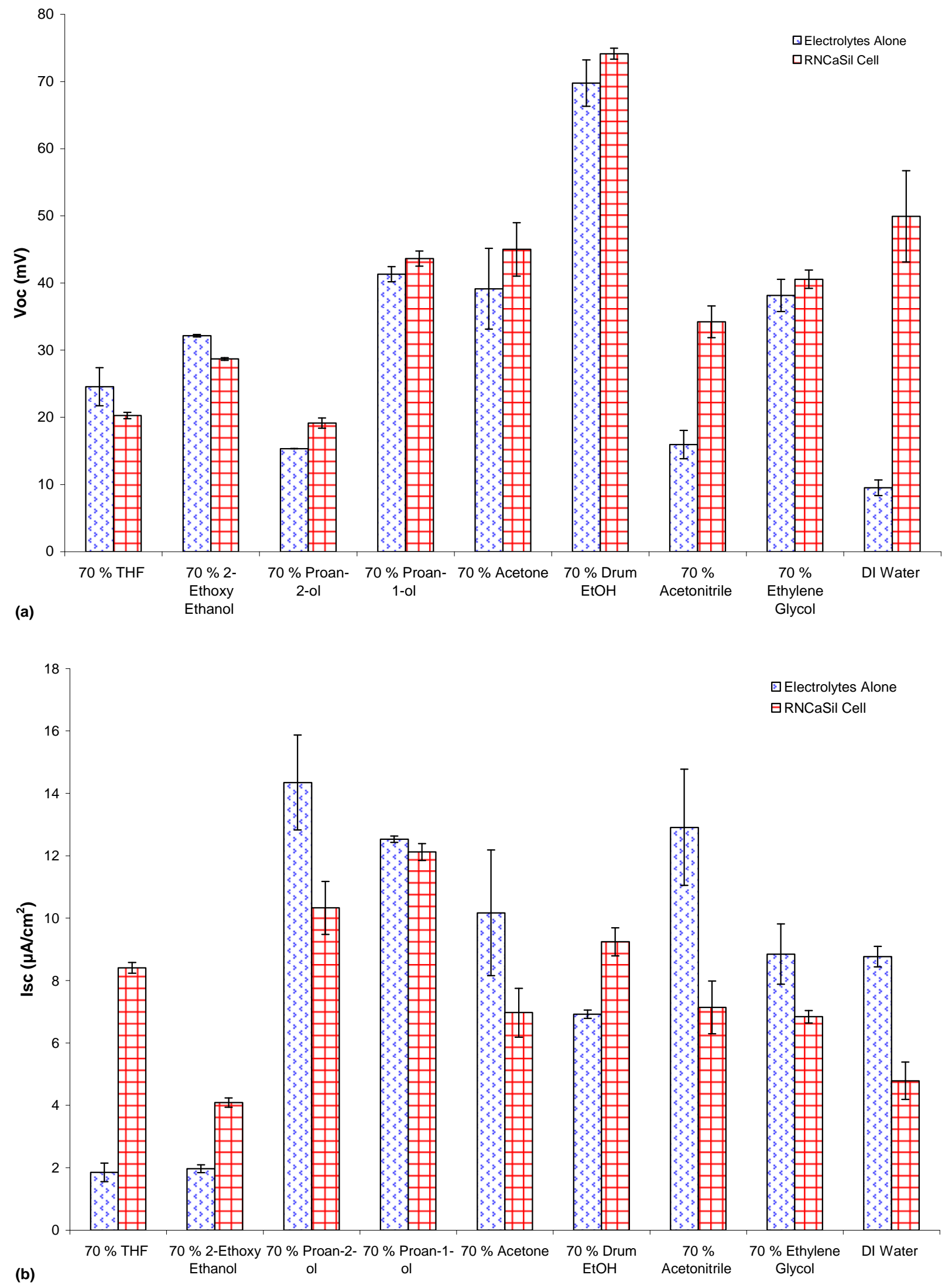

Figure 48. The Effect of Iodide/Triiodide Electrolyte Solvent - Plotted in Order of Increasing Dielectric Constant of the Dominant Solvent: (a) $V_{o c}$ and (b) $I_{s c}$. The Secondary Solvent (30 wt\%) was Water. 
From this point onwards, the focus of the experimental work shifted to the $70 \mathrm{wt} \%$ ethanol iodide/triiodide electrolyte, due to the formation of a gel from such an electrolyte with RNCaSil, which showed superior performances. Hence, the effect of the different solvents was not explored further.

\subsubsection{The Special Case of Ethanol}

Ethanol is a favourable solvent because - unlike other solvents popular with DSSCs, such as acetonitrile - ethanol is non-toxic and environmentally benign. ${ }^{[90]}$ Ethanol interacted with RNCaSil in a way which was conducive to cell performance and which had not been observed with other solvents. Importantly, it was one of the few solvents where the addition of $\mathrm{RNCaSil}$ to its electrolyte (no $\mathrm{CaCl}_{2}$ added) increased cell efficiency (above, Figure 47). With RNCaSil, it showed both greater cell potential and current. Additionally, as is discussed further below (Part IV 8.1), it was the only solvent tested which formed a gel with RNCaSil.

Kebede et al. ${ }^{[60]}$ stated that the interaction between iodine and a solvent can be observed by a blue-shift in the absorption spectra. This was observed for the $70 \mathrm{wt} \%$ ethanol iodide/triiodide electrolyte, as compared to a water-based electrolyte (above, Figure 28), and explained why slightly higher potentials were usually obtained in the ethanol-based electrolyte. They further stated that such an interaction encouraged the formation of triiodide:

Equation 8. Solvent $\mathrm{I}^{+} \mathrm{I}^{-} \leftrightarrow \quad$ Solvent $\mathrm{I}^{+}+\mathrm{I}^{-}$

Equation 9. Solvent $\mathrm{I}^{+} \mathrm{I}^{-}+\mathrm{I}^{-} \quad \leftrightarrow \quad \mathrm{I}_{3}{ }^{-}+$Solvent

As triiodide is the photoactive species, its increased concentration raises cell performance. Further, the rise in ions present causes the conductance to rise. ${ }^{[60]}$

\subsubsection{Long-Term Stability}

Difficulties in sealing off gasket cells for long periods of time resulted in evaporation of the solvent, the amount of which was large compared to the initial sample size, further making maintenance of electrical contact problematic. Consequently, long-term tests of RNCaSil and iodide/triiodide electrolytes prepared in different solvents ( $70 \mathrm{wt} \%$ other and $30 \mathrm{wt} \%$ water), were undertaken in beakers, in the hope that any loss of solvent would be minimal compared to the bulk sample. These beakers tests showed a large solvent effect on cell performance over 72 hours (Figure 49). These initial 
tests were not carried out under constant illumination, as they sought to determine whether the samples were capable of being used even if prepared some time before being tested. Testing beyond 72 hours, under constant illumination, is discussed in depth below (Part IV 8.1).
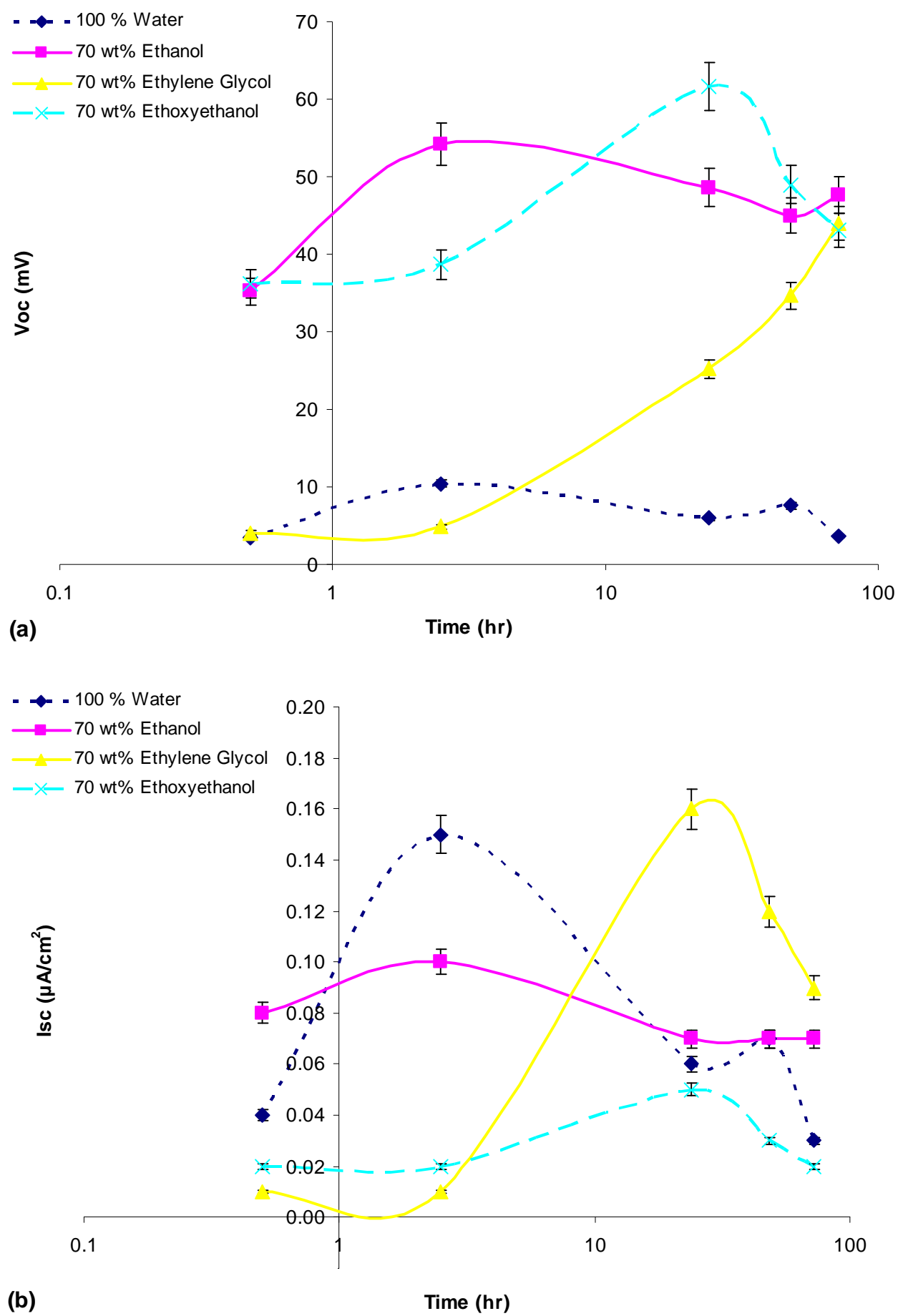

Figure 49. Beaker Tests of RNCaSil in Differently Solvated Iodide/Triiodide (30 wt \% water:70 wt\% other); (a) $V_{o c}$, and (b) $I_{s c}$. Non-constant Illumination. 
Both the current and potential of cells varied significantly over time. The pattern of variation depended on the solvent used in the electrolyte (Figure 49). Notably unstable were the water and $70 \mathrm{wt} \%$ ethylene glycol-based RNCaSil cells. The ethylene glycol cell barely functioned initially, and it was not until 2.5 hours had passed that the current and potential started to rise. Compared to the other samples, the ethylene glycol cell showed the largest increase in $V_{o c}$ and $I_{s c}$ over the 72 hours. This was attributed to the high viscosity of ethylene glycol, which made electron transport through the larger testing vessel difficult and required a longer period of time to initiate. However, after 24 hours, the potential still rapidly rose, while the current started to decline sharply. The water based cell was equally as unstable. It was not known why the potential of the water-based sample was so low in this case. Other beaker tests with RNCaSil and water-based electrolyte did not exhibit such low voltages. Potentials being low (i.e., spikes far out from the average) have been noted on other occasions (for example, Part IV 4.3.1). In any case, the current of the sample proved insignificantly low and unstable.

The 70 wt\% ethoxyethanol-containing cell also showed large variations between data points. The sample additionally had a very low cell performance, which was also observed in gasket cells, as in the above section (Part IV 4.4.1). The reason that ethoxyethanol made a poor solvent for NCaSil cells was that it was readily absorbed onto NCaSils, whereupon it blocked up its pores. Such a large affect was not observed for EENCaSil, as the material only contained approximately $3 \%$ ethoxyethanol.

It was only the $70 \mathrm{wt} \%$ ethanol based cell that showed stability and that did not degrade over time. The good performance of the ethanol-based cells was indicative of the fact that the solvent had favourable interactions with RNCaSil, as compared to other solvents. This was supported by the later finding that, over time, such samples formed much more highly active gels, whereas samples made up from other solvents did not (Part IV 8.1).

\subsubsection{Addition of $\mathrm{CaCl}_{2}$ to $70 \mathrm{wt} \%$ Ethanol Iodide/Triiodide Electrolyte}

Earlier testing had shown that the addition of $\mathrm{CaCl}_{2}$ to the water-based iodide/triiodide electrolyte increased cell current both of the electrolyte alone and when used with RNCaSil (Part IV 4.3.1). The effect of adding $\mathrm{CaCl}_{2}$ to the $70 \mathrm{wt} \%$ ethanol-based (30 wt $\%$ water) iodide/electrolyte is discussed here. The differences between the water-based and $70 \mathrm{wt} \%$ ethanol-based iodide/triiodide electrolytes were made more obvious when both electrolytes were tested with and without 1.5 or $2 \mathrm{wt} \% \mathrm{CaCl}_{2}$, and with and without the presence of RNCaSil, in normal gasket cells. 
The ethanol electrolyte cells had consistently higher voltages than their water-based counterparts (Figure 50(a)). It was interesting to note that usually the potential increased on the addition of $\mathrm{CaCl}_{2}$ to the pure water-based electrolyte. However, this was not the case with the ethanol-based iodide/triiodide cells; both with and without RNCaSil, the potential dropped on the addition of $2 \mathrm{wt} \% \mathrm{CaCl}_{2}$.
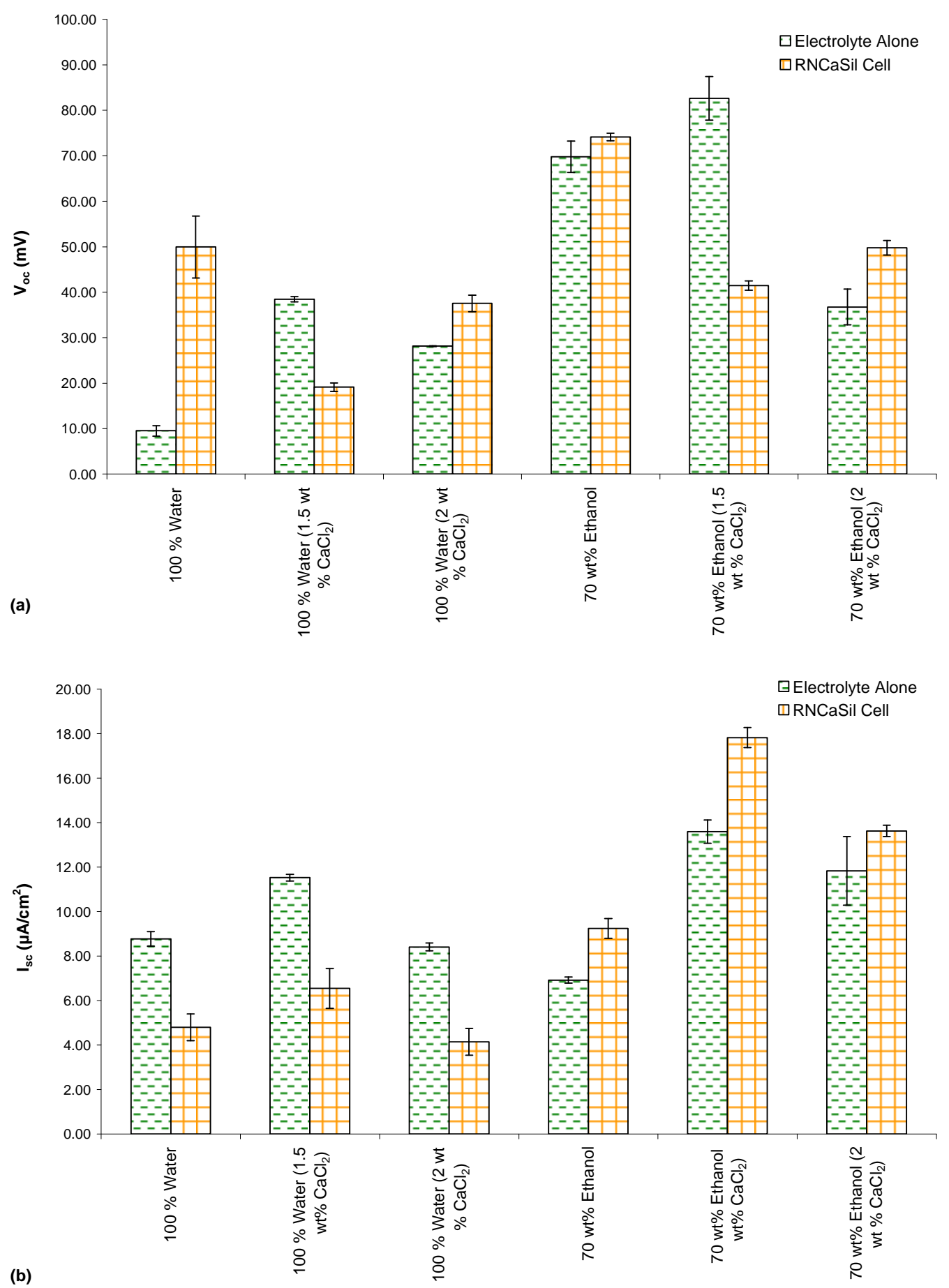

Figure 50. The Effect of Adding $2 \mathrm{wt} \% \mathrm{CaCl}_{2}$ to the Electrolyte of Water or $70 \mathrm{wt} \%$ Ethanol (30 \% Water) Iodide/Triidide Electrolytes, With and Without RNCaSil (Normal Gasket); (a) $V_{o c}$ and (b) $I_{s c}$. 
Also of note was that the ethanol-based cells always showed enhanced cell current on the addition of RNCaSil and this was further increased with the use of 1.5 or $2 \mathrm{wt} \% \mathrm{CaCl}_{2}$ in the electrolyte (Figure 50(b)). Comparatively, the water-based cells showed a loss of current when used in conjunction with $\mathrm{RNCaSil}$, both with and without 1.5 or $2 \mathrm{wt} \% \mathrm{CaCl}_{2}$.

In actual fact, the $2 \mathrm{wt} \% \mathrm{CaCl}_{2}$ did not fully dissolve into the electrolyte, even after stirring for several hours. It was estimated that only around $1.5 \mathrm{wt} \%$ actually dissolved. $1.5 \mathrm{wt} \%$ of $\mathrm{CaCl}_{2}$ in the $30 \mathrm{wt} \%$ water electrolyte is equivalent to $5 \mathrm{wt} \%$ in the water portion. This was particularly interesting when one considered that $5 \mathrm{wt} \%$ seemed to be the "magical" $\mathrm{CaCl}_{2}$ content in the $100 \%$ water based iodide/triiodide electrolyte; where the addition of RNCaSil increased current rather than inhibiting it (Part IV 4.3.1). Therefore, there appeared to be a driving force for having $5 \mathrm{wt} \% \mathrm{CaCl}_{2}$ in the water, regardless of the fact that the water was only $30 \mathrm{wt} \%$ of the electrolyte solvent. This was supported by the results obtained when $1.5 \mathrm{wt} \% \mathrm{CaCl}_{2}$ was used, the entirety of which dissolved into the electrolyte solution. The greatest current achieved here was with $1.5 \mathrm{wt} \% \mathrm{CaCl}_{2}$ added to the iodide/triiodide electrolyte and used in conjunction with RNCaSil. That the $2 \mathrm{wt} \%$ sample did not work as well was also not surprising given that, above $5 \mathrm{wt} \%, \mathrm{Ca}(\mathrm{OH})_{2}$ precipitated (Part IV 4.3.1).

\subsection{Dark Electrolyte Process}

In some solvents, the iodide/triiodide electrolyte alone and with RNCaSil some times exhibited a dark process, which was either "forward" (in the same direction as the light induced process) or "backward" (Figure 51). It was suspected that in all cases, the observed process was due to the iodine/iodide/triiodide species reaching equilibrium, as the processes diminished over time (though the solutions were made 24 hours prior to use in cells). The variance of iodide and triiodide content is known to depend on cell characteristics. ${ }^{[77]}$ There was no clear trend as to why certain solvents allowed for such dark processes and why some were forward and others backward. As the processes faded over time and these observations were not pertinent to the project, it was not investigated any further. It did, however, highlight the importance of long-term testing, which would have allowed for the electrolytes to come to equilibrium upon the addition of NCaSil. 

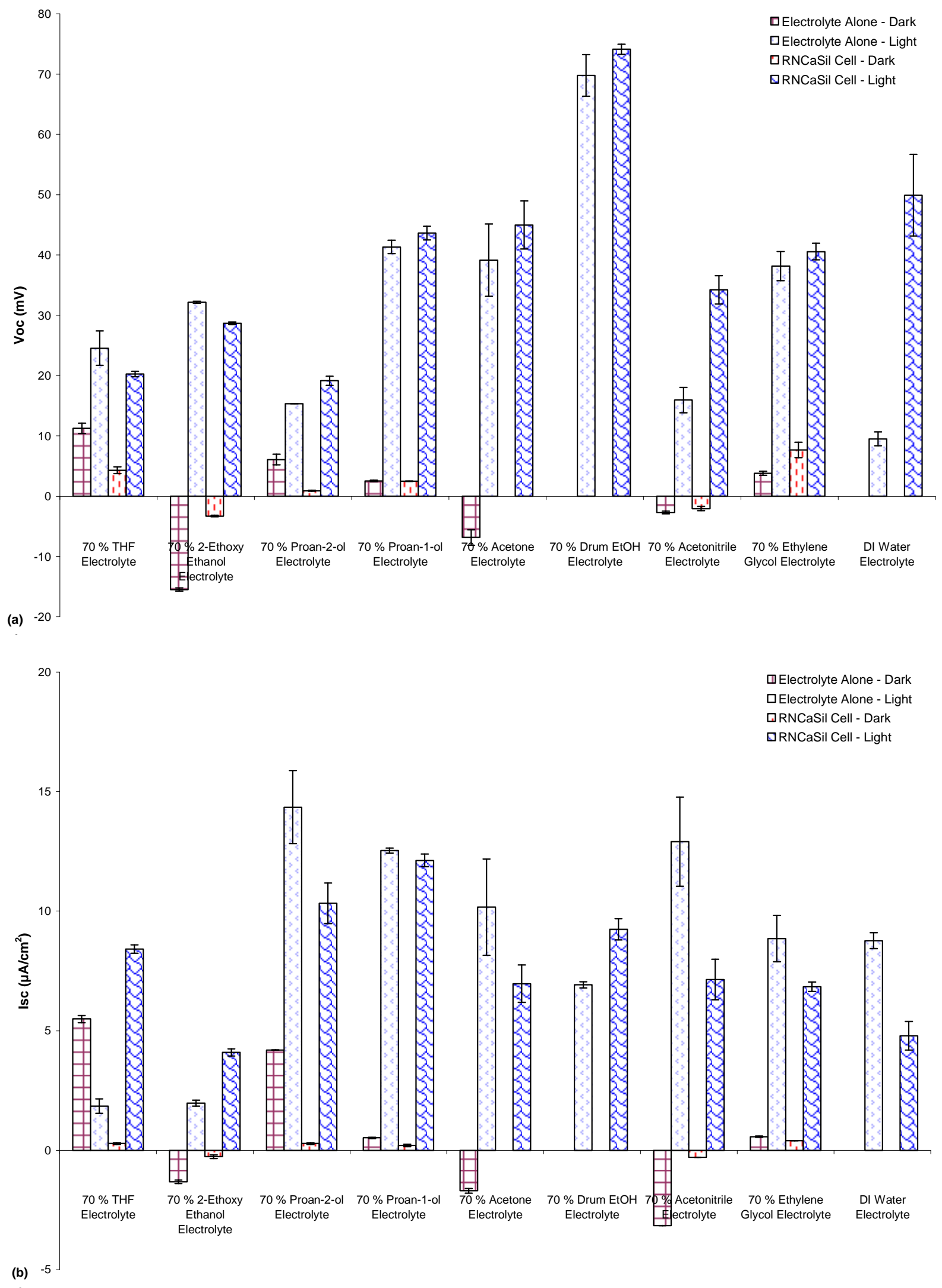

Figure 51. Effect of Solvent on RNCaSil Cells in $30 \mathrm{wt} \%$ Water Iodide/Triiodide, Including Dark Processes. Plotted in Order of Increasing Dielectric Constant of the Solvent; (a) $V_{o c}$ and (b) $I_{s c}$. 


\section{Effect of Cell Surface Area and Sample Depth}

In this chapter, the effect on RNCaSil and iodide/triiodide electrolyte cells of changing the cell surface area and cell depth is examined. The effect of cell surface area has important consequences on whether a cell could be scaled up for commercial use. It was expected that the cell performance would increase on widening the cell, as the surface area was increased. Barring any electrode effects, the rise in current should, ideally, have been linear relative to the increase in surface area. With regards to electrode distance, it has been observed with $\mathrm{TiO}_{2}$ DSSCs that there is an optimal electrode distance (Part I 1.3.1), whereas semiconducting photogalvanic cells only decrease in performance on increasing cell depth (Part I 3.1), and thin-layer-electrolyte photogalvanic cells have shown very little dependence on electrode distance (Part I 3.2). Therefore, the behaviour exhibited on changing electrode distance was also expected to be indicative of cell type.

\subsection{Cell Surface Area}

The same Teflon used to construct the normal gaskets was used to test the effect of cell width and surface area. This meant that the cell depth remained constant, but the surface area of the cell changed. As the cell diameter increased, there appeared to be a slight increase in voltage (Figure 52(a)). This was not expected and was attributed to experimental uncertainty.

As to the current, an unexpected pattern was observed on changing the gasket width (Figure 52(b)). One would have expected that the current, as plotted in $\mu \mathrm{A} / \mathrm{cm}^{2}$, would either have risen as the surface area increased, or the current should have fallen, as recombination may have been disproportionately enhanced, due to electrode effects. Instead, a surface area maximum was observed. It was not thought that the data obtained from the gaskets of $5.05 \mathrm{~mm}$ diameter were due to experimental error. Being the gasket diameter used for most experiments, the results for the $5.05 \mathrm{~mm}$ diameter gasket were within the range expected. This range was quite wide due to its relatively small area, resulting in greater experimental uncertainty.

This strange and non-DSSC behaviour was indicative of a dominating photogalvanic process. The results also suggested that scalability may be an issue. 

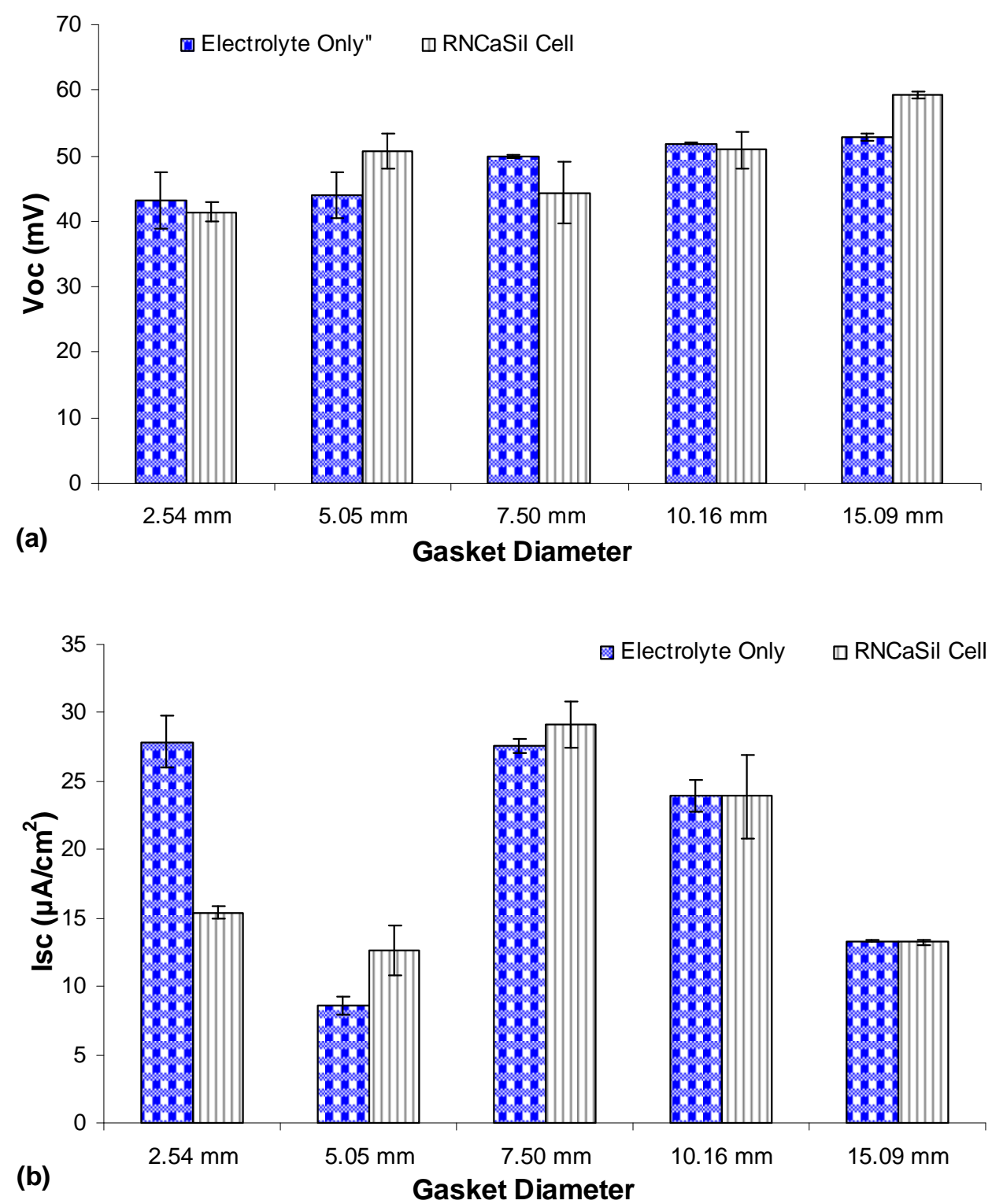

Figure 52. The Effect of Cell Diameter on Cell Performance of RNCaSil in Water-Based Iodide/Triiodide (5 wt \% $\mathrm{CaCl}_{2}$ ) Electrolyte; (a) $V_{o c}$ and (b) $I_{s c}$.

\subsection{Cell Electrode Distance}

\subsubsection{Different Gasket Depths}

Teflon tape ("Ceelon") was used, with a thickness of $0.08 \mathrm{~mm}$ (herein called "0.08 mm gaskets"), compared to the normal gaskets which had a thickness of $0.52 \mathrm{~mm}$. The volume ratio of the normal gasket to Teflon tape gasket was 46:1. The sample surface area of the Teflon tape cells $(6.00 \mathrm{~mm}$ diameter, $1.13 \mathrm{~cm}^{2}$ ) was not quite the same as that of the "normal" gaskets $(5.05 \mathrm{~mm}$ diameter, 0.80 $\mathrm{cm}^{2}$ ). However, it was expected that the much larger difference in cell depth would dominate the 
smaller variance in sample surface area and, thus, any divergence observed would be due to the changes in cell depth.

It is prudent to note at this point the (sometimes) large uncertainties that the NCaSil cells showed, due to inconsistencies in the sample, for example, because of its amorphous nature and the method of sample manufacture (as discussed earlier, Part IV 1.3). This was more likely with the $0.08 \mathrm{~mm}$ gaskets, due to their low volume. The small sample size made it difficult to control the exact ratio of RNCaSil:electrolyte, as excess electrolyte could be pushed out sometimes during cell construction, increasing the proportion of excess RNCaSil.

The pattern exhibited in the normal gaskets on adjusting the RNCaSil content has already been discussed (Part IV 4.1). What was interesting here was that, when in thinner gaskets, the pattern was not the same. Unlike the normal gaskets, there was a clear trend of increasing voltage as the ratio of RNCaSil to electrolyte increased, with a spike at a load of $0.05 \mathrm{~g}$ RNCaSil (Figure 53(a)). The upwards trend for the normal gaskets was subtle and within experimental uncertainty. The voltages were always lower for the $0.08 \mathrm{~mm}$ gasket cells than the normal gasket cells. This disagreed with results obtained by Hara et al. ${ }^{[16]}$, who found that, using a mercurochrome sensitiser with a $\mathrm{TiO}_{2}$ cell, as the cell thickness increased, $V_{o c}$ decreased. This, again, indicated that NCaSil cells were not pure DSSCs.

The $I_{s c}$ exhibited a similar pattern in the $0.08 \mathrm{~mm}$ gasket cells as in the normal cells, in that there was also a maximum $I_{s c}$ (Figure 53(b)). It was interesting that there was no difference between the two gasket depths with the electrolyte solution alone. It was expected that as, in either case, the light source was easily able to pass through the whole sample, the gasket with more sample would display a higher current in a photogalvanic cell. The similar currents were attributed to the fact that, though the normal gaskets had more sample to be activated, the ions also had a greater distance to travel between electrodes. The current of the $0.08 \mathrm{~mm}$ gasket cells peaked between 0.05 and $0.10 \mathrm{~g} / \mathrm{cm}^{3}$ loadings of RNCaSil, before declining. As discussed earlier, with regards to the normal gasket cells (Part IV 4.1), this observed reduction in current was expected, due to higher proportions of the ions (and solvent) of the electrolyte being sorbed onto the surface of the RNCaSil, rendering them immobile.

With respect to the current, from the literature on DSSCs, one would have expected the current to be either higher or lower for all the $0.08 \mathrm{~mm}$ gasket samples, compared to the thicker normal gasket cells. Ito et $a l .{ }^{[53]}$ and Hara et al. ${ }^{[16]}$ both found that $I_{s c}$ rose as a cell's thickness increases up to a certain maximum (as there was more sample), before falling off. Beyond that maximum point, the $I_{s c}$ fell off as 
the distance that the electrons and holes had to travel became too great. ${ }^{[10]}$ For the 0.01 and $0.05 \mathrm{~g} / \mathrm{cm}^{3}$ RNCaSil loads, the current was greater for the $0.08 \mathrm{~mm}$ gasket cells than the normal gasket cells, which was likely due to easier diffusion at lower cell depths and the fact that the light was able to pass through the entire sample. However, this was reversed for the greater three RNCaSil loads. This was attributed to the fact that, at these higher loadings, the thinness of the cells was no longer as relevant. As the density increased, less light was likely to pass through the entire cell, and as much light was likely caught by the sample closest to the light source in $0.08 \mathrm{~mm}$ gasket cells as in normal gasket cells.
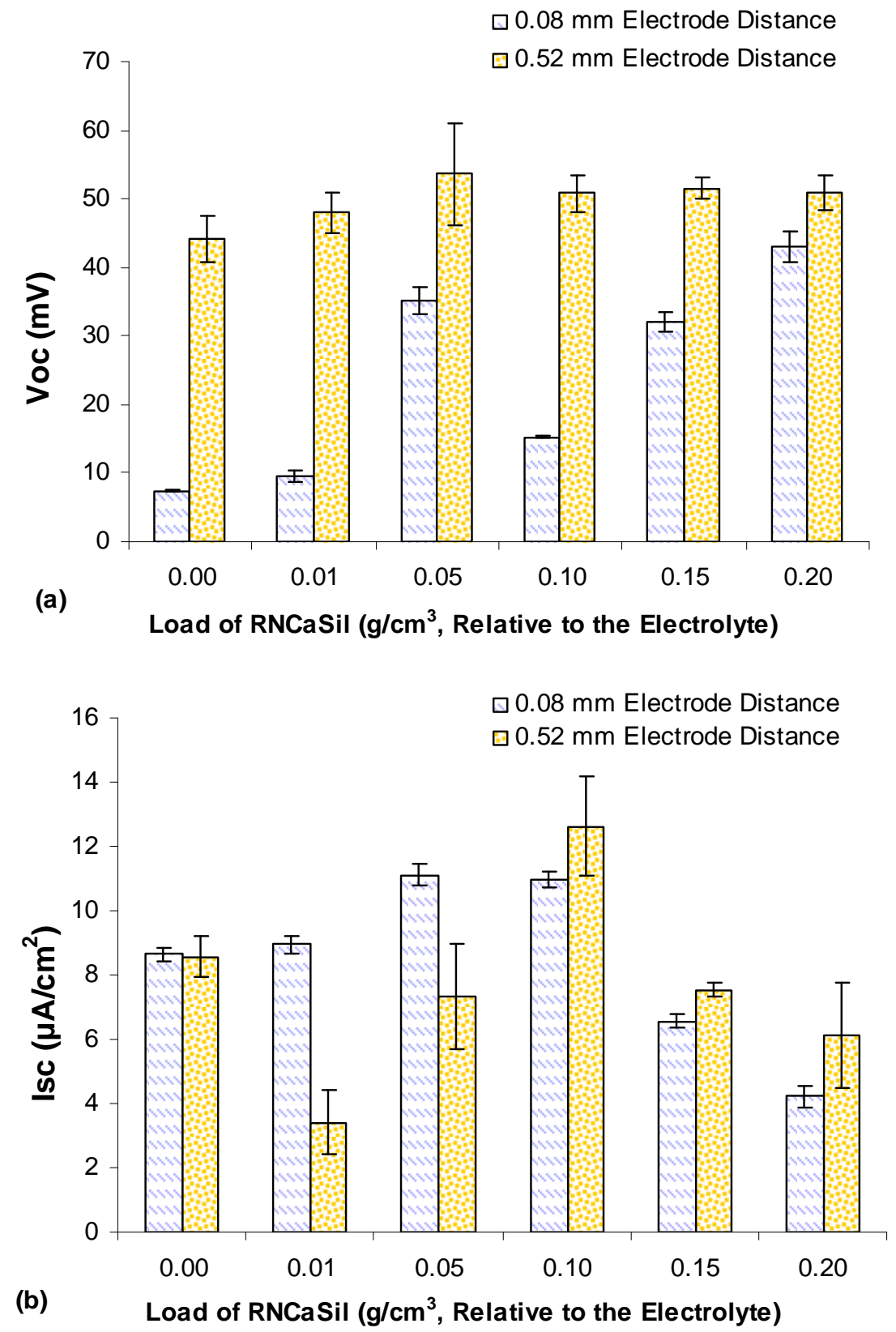

Figure 53. Effect of Electrode Distance with Different Loads of NCaSil to $1.0 \mathrm{~cm}^{3}$ Water-Based Iodide/Triiodide $\left(5 \mathrm{wt} \% \mathrm{CaCl}_{2}\right.$ ) Electrolyte; (a) $V_{o c}$ and (b) $I_{s c}$. 
Thus, the results here were inconclusive. However, it appeared that the normal gaskets were closer to the optimal operating depth than the $0.08 \mathrm{~mm}$ gaskets. It was arguable that the disagreeing results of the $0.08 \mathrm{~mm}$ gasket samples in 0.01 and $0.05 \mathrm{~g} / \mathrm{cm}^{3}$ loadings were due to experimental errors. More exact methods would need to be developed to fully ascertain this. That some of the $0.08 \mathrm{~mm}$ gasket cells had lower currents meant that the cell process was not predominantly diffusion controlled, at least not at these two depths, which suggested non-DSSC behaviour. Furthermore, it was indicative that the cell was not acting as a semiconducting photogalvanic cells. Tentatively, the lack of a clear trend suggested thin-layer-electrolyte photogalvanic cells.

More pertinent was the comparison of the two gasket depths with respect to $I_{s c}$ relative to volume, rather than surface area alone. Given the small samples in the $0.08 \mathrm{~mm}$ gasket compared to the normal gaskets, the cells functioned very well. (Figure 54). The $I_{s c}$ in $\mu \mathrm{A} / \mathrm{cm}^{3}$ of the $0.08 \mathrm{~mm}$ gasket cells were, at best, seventeen times greater than those of the normal gasket cells. This showed how poorly the RNCaSil particles were connected to each other and the electrode in the normal gasket cells.

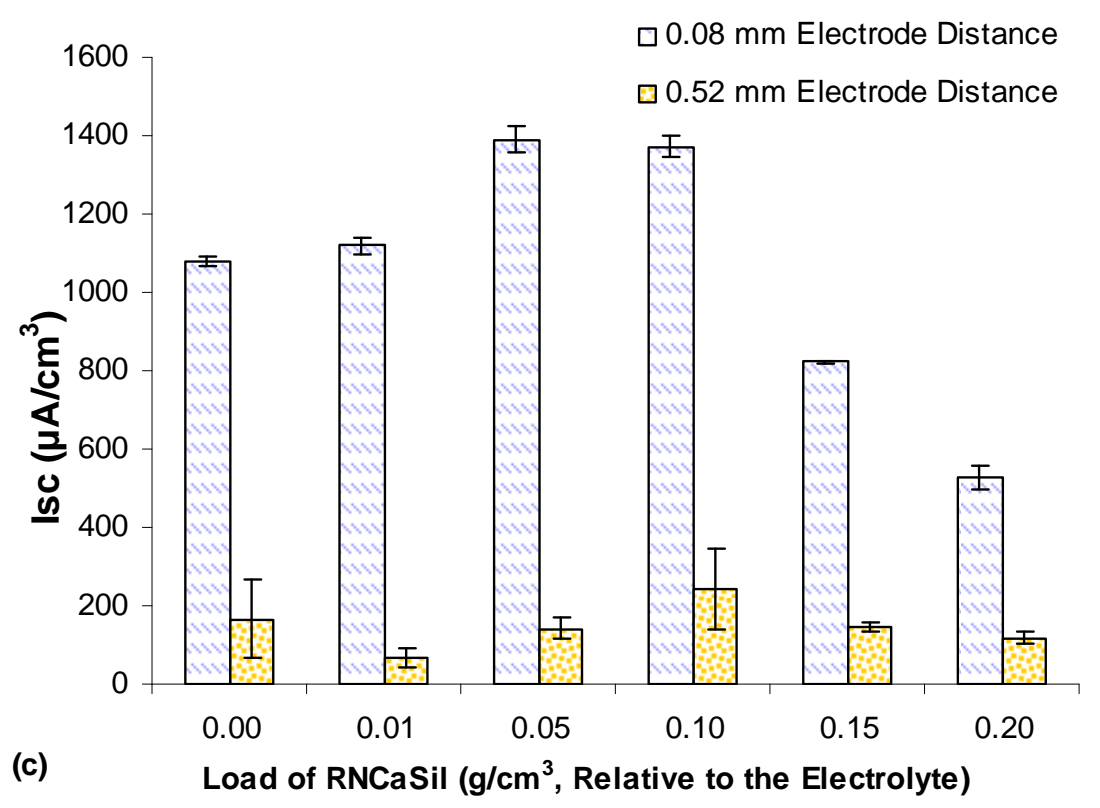

Figure 54. Effect of Electrode Distance with Different Loads of NCaSil to $1.0 \mathrm{~cm}^{3}$ Water-Based Iodide/Triiodide $\left(5 \mathrm{wt} \% \mathrm{CaCl}_{2}\right)$ Electrolyte, $I_{s c}\left(\mu \mathrm{A} / \mathrm{cm}^{3}\right)$.

\subsubsection{Electrode Distance in Beaker Cells}

Results obtained from beaker cells were not directly comparable to those obtained in gasket cells, as the cells were affected by the curvature of the glass, a larger electrode surface area, the presence of 
sample between the beaker wall and the photoelectrode and the generally bulk nature of the beaker cells. In a beaker test with RNCaSil and water iodide/triiodide $\left(5 \mathrm{wt} \% \mathrm{CaCl}_{2}\right)$ electrolyte, the distance between the electrodes was modified to approximately $0.5,2.0$ and $2.5 \mathrm{~cm}$.

The results showed a pattern of increasing current as the electrode distance was widened (Figures 55 and 56). The same trend was shown for beaker cells, where the electrode position relative to the light was modified - namely with the electrodes parallel to the light beam or with the coated electrode closest to the light - though the values obtained were lower. It appeared that the potential was also enhanced on widening the electrode distance, however the results from the beaker cells with modified electrode positions, relative to the light, showed that there was no such general trend. On the contrary, the change in $V_{o c}$ was quite haphazard.

These results again indicated that the current of these cells was not diffusion controlled and not behaving as DSSCs or semiconducting photogalvanic cells, but rather as thin-layer-electrolyte photogalvanic cells.

The effect of the different electrode positions is discussed in depth below (Part IV 7.3).

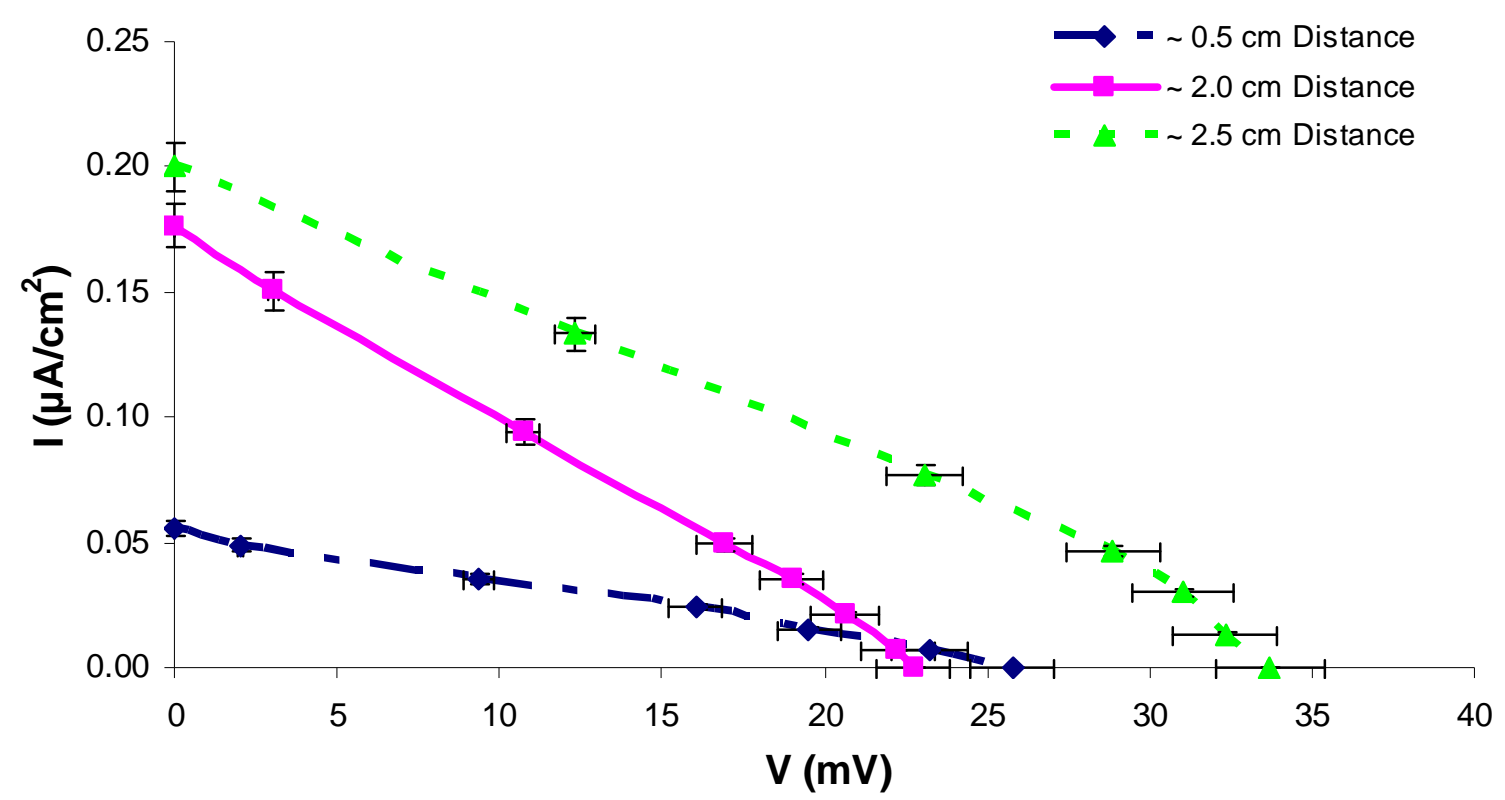

Figure 55. Effect of Electrode Distance on a RNCaSil and Water-Based Iodide/Triiodide (5\% $\mathbf{C a C l}_{2}$ ) Electrolyte Beaker Test (Ratio of $1.0 \mathrm{~g}: 10 \mathrm{~cm}^{3}$ ). Normal Set Up; the Electrodes were Perpendicular to the Light Beam with the C-coated ITO as the Dark Electrode. 

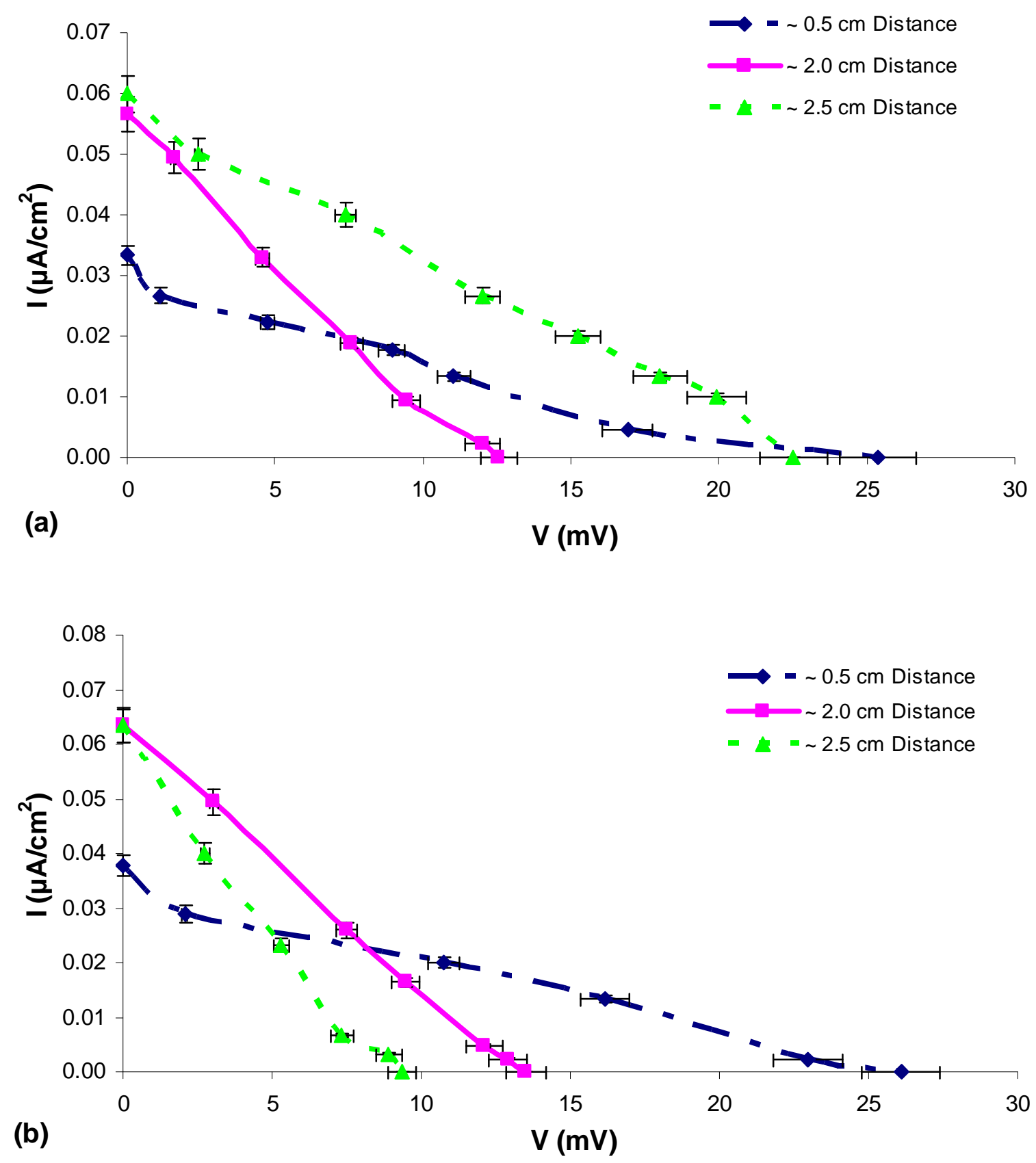

Figure 56. Effect of Electrode Distance on a RNCaSil and Water-Based Iodide/Triiodide (5\% $\mathbf{C a C l}_{2}$ ) Electrolyte Beaker Test (Ratio of $1.0 \mathrm{~g}: 10 \mathrm{~cm}^{3}$ ): (a) Electrodes Parallel to the Light Beam; and (b) Electrodes Perpendicular to the Light Beam with the C-coated ITO as the Photoelectrode.

\subsubsection{Slotted-Vessel Tests}

RNCaSil with iodide/triiodide was tested in a slotted vessel to assess the effect of electrode distances. Because there was a tendency for the RNCaSil cells to increase in current and voltage over time, particularly in the first hour of illumination, these samples were left under the lamp for an hour to stabalise. C- and Pt-coated ITO glass, and Pt-wire back electrodes were tested. Also investigated was 
the effect of the sample between the quartz glass of the slotted-vessel and the photoelectrode (Figure 57), which was not expected to have been partaking in any photocell process. The sample was removed from this space resulting in a "gap" and the cell tested.

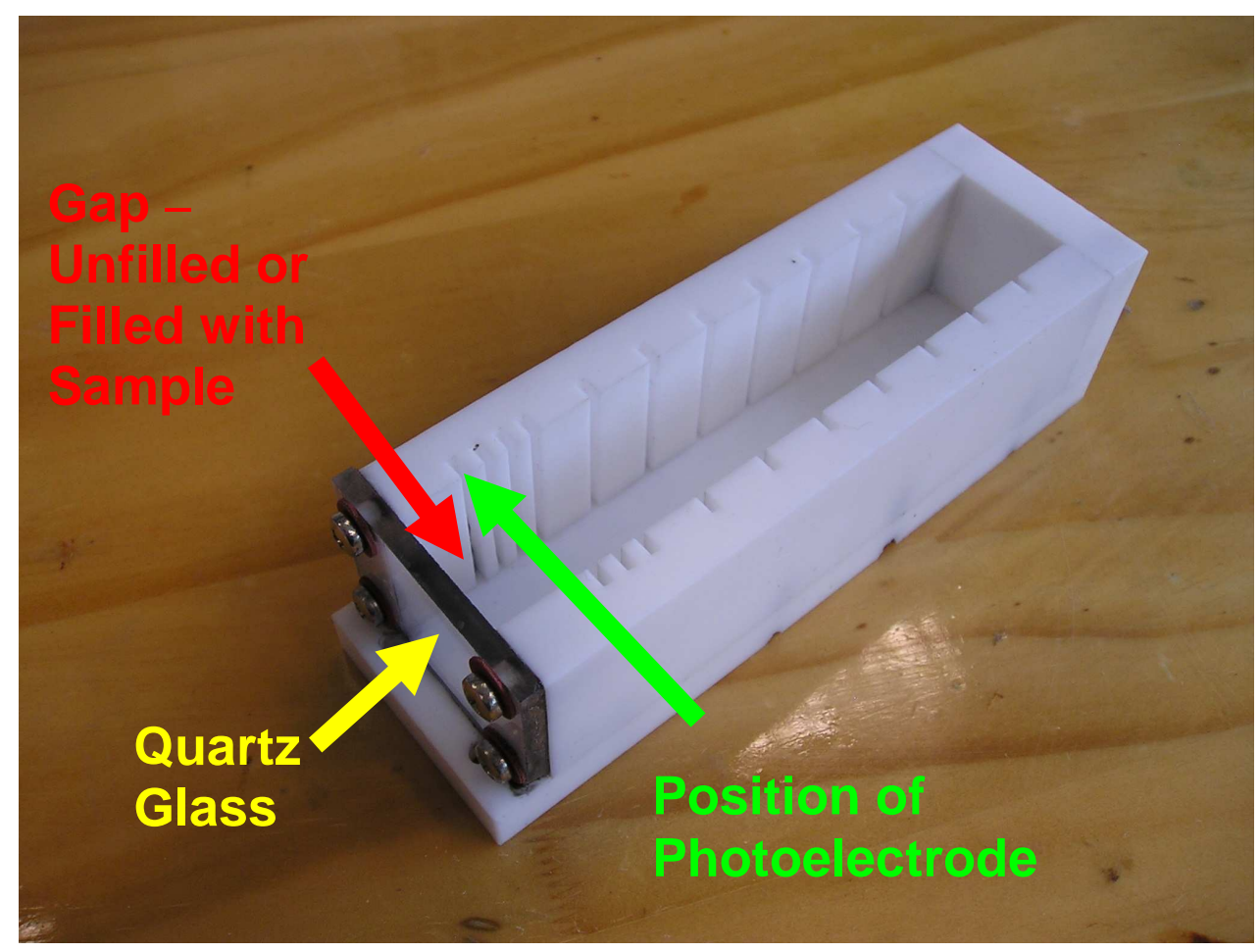

Figure 57. Slotted Vessel - The Gap Between the Quartz Glass and Photoelectrode can be Unfilled or Filled with Sample.

Some of the distances tested in the slotted-vessel were much larger than those in any of the previous tests mentioned. Consequently, it was expected that a clear trend would be observed. However, this was not the case (Figure 58). Both the $V_{o c}$ and $I_{s c}$ appeared indifferent to the distance between the electrodes, which suggested the dominance of a thin-layer-electrolyte photogalvanic process. However, even in an iodide/triiodide dominated photogalvanic cell, some variance was expected with changes in electrode distance. ${ }^{[106]}$ Thus, the process was not purely photogalvanic.

Regardless of the back-electrode coating used, there was no observable trend in potential on changing electrode distance. It could be argued that there were slight increases in voltage as the electrode gap widened. However, this was minimal and likely within experimental uncertainty, particularly given the propensity for the cells to drift over time. The voltages exhibited by the C-coated ITO glass back electrode sample were higher than those normally exhibited by other such composed cells previously investigated. It was suspected that this was due to the C-coating being removed from the ITO and 
entering into the sample, as was visibly observed. This was not observed with the Pt-coated back electrode sample and was attributed to the greater stability of platinum over carbon.

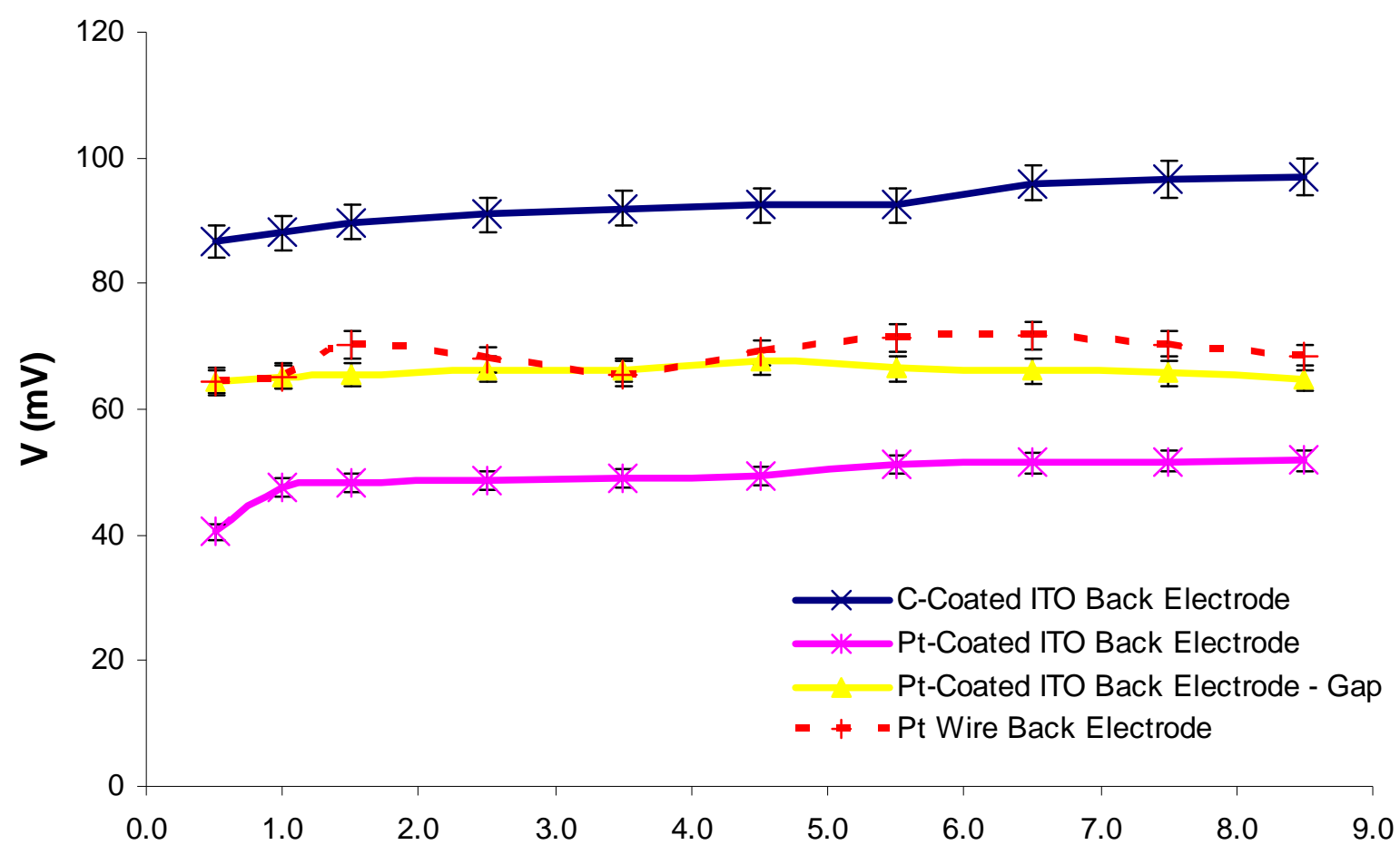

(a)

Distance (cm)

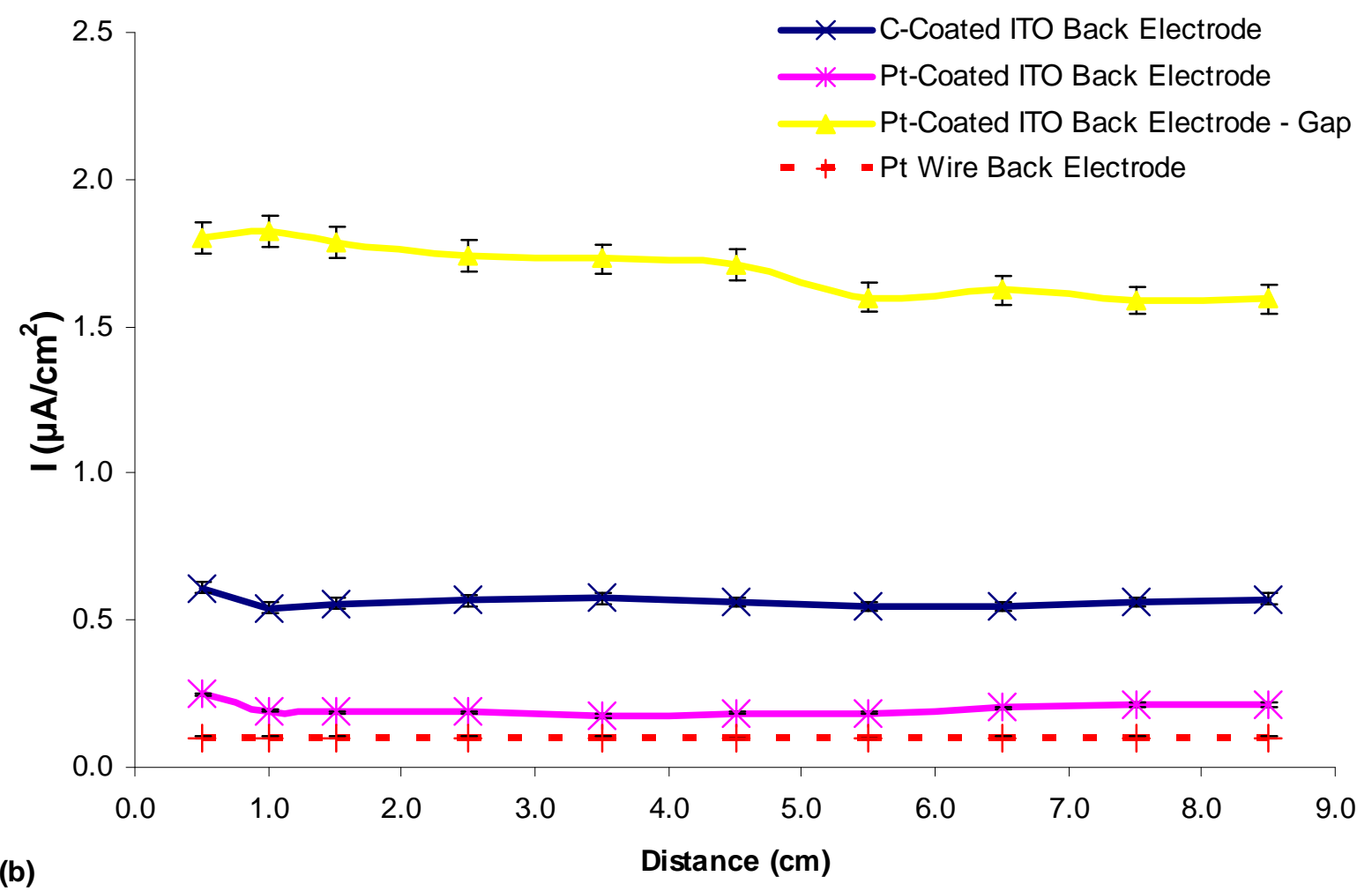

Figure 58. Affect of Distance on (a) $V_{o c}$ and (b) $I_{s c}$ on RNCaSil in 70 wt $\% 30$ wt $\%$ Water-Based Iodide/Triiodide Electrolyte. 
As with the potential, with any of the samples tested, there was no general trend with respect to the current, as the electrode distance was modified. Why this was the case was not resolved. There was a small downward pattern observable with the Pt-coated ITO back electrode sample, with no sample between the quartz glass on the vessel and the front ITO electrode. However, as the other three samples showed no such a trend, this was attributed to the general drift in current that was often observed with bulk cells (for example, see Part IV 3.2). That the Pt-coated ITO back electrode sample did not achieve as great a current as the C-coated back electrode sample was also thought to be due to the removal of carbon from the coating into the sample.

That the Pt-coated ITO back electrode sample, with the gap between the quartz glass on the vessel and the front ITO electrode, showed such an improved current over the other tests with samples filling that gap, was interesting for unrelated reasons. It indicated that having sample between the light source and front electrode inhibited the light path. This was confirmed by tests which removed the surrounding sample of beaker cells from gel samples between the electrodes, as discussed below (see Part IV 9.3).

\subsubsection{Conclusions on Electrode Distance}

It appeared that, at shorter distances, there was an effect on cell current. That the $I_{s c}$ increased with widening of the cell was observed in both gasket and beaker cells. There was no apparent trend for the $V_{o c}$. However, testing in the slotted-vessel exhibited neither a trend in the current or potential, as the electrode distance was changed, and there appeared to be no difference in $I_{s c}$ or $V_{o c}$ whether the electrodes were 0.5 or $8.5 \mathrm{~cm}$ apart. Why this was the case was not known, but clearly indicated that cells composed of RNCaSil and iodide/triiodide were neither purely DSSC or photogalvanic in nature. They were undoubtedly not diffusion controlled. In DSSCs, one would have expected an electrode distance where the $I_{s c}$ and $V_{o c}$ peaked; the current and potential both being affected by cell depth. Both semiconducting and thin-layer-electrolyte photogalvanic cells should also have been affected by electrode distance, though the latter to a lesser degree, as this determined the sample volume. That the RNCaSil-containing cells did not fall exactly into any of these categories suggested that soggy sand electrolytes had been made. 


\section{Back-Reflection}

While doing tests to ascertain the effect of cell depth, the white Teflon tape used as a thin gasket was wound around the entire dark electrode of some cells. In other words, these cells had a white backing, which reflected light back into the cell. With "un-backed" cells, any light that passed through the sample and (coated) back electrode would have dissipated in the black box the cells were tested in.

As cell potential is determined by the different energy levels of the species involved in the photoactive process, there should have been no trend exhibited by the cell potential dependant on whether the cells were backed or not. This did not appear to be the case here (Figure 60(a)). It was not known why the voltage increased with cell backing of the $0.08 \mathrm{~mm}$ thick cells, however it was suspected that much of this was due to experimental uncertainty. The $V_{o c}$ of the RNCaSil cells tended to fluctuate, somewhat inconsistently, between 35 and $55 \mathrm{mV}$. For example, no increase was observed on backing a normal $0.52 \mathrm{~mm}$ thick gasket cell (of 0.10 loading of RNCaSil) with Teflon tape and a $V_{o c}$ of $50.7 \pm 4.8 \mathrm{mV}$ was observed, which was comparable to that of a non-backed cell $(50.8 \pm 2.7 \mathrm{mV})$. This variation was likely due to the amorphous and changeable nature of NCaSil. Alternatively, it could be speculated that the increases were due to secondary absorbances of wavelengths, not absorbed during the first pass of the light, placing the excited electron in a band with a more negative (vs. NHE) potential, thus increasing $V_{o c}$ (Figure 59).

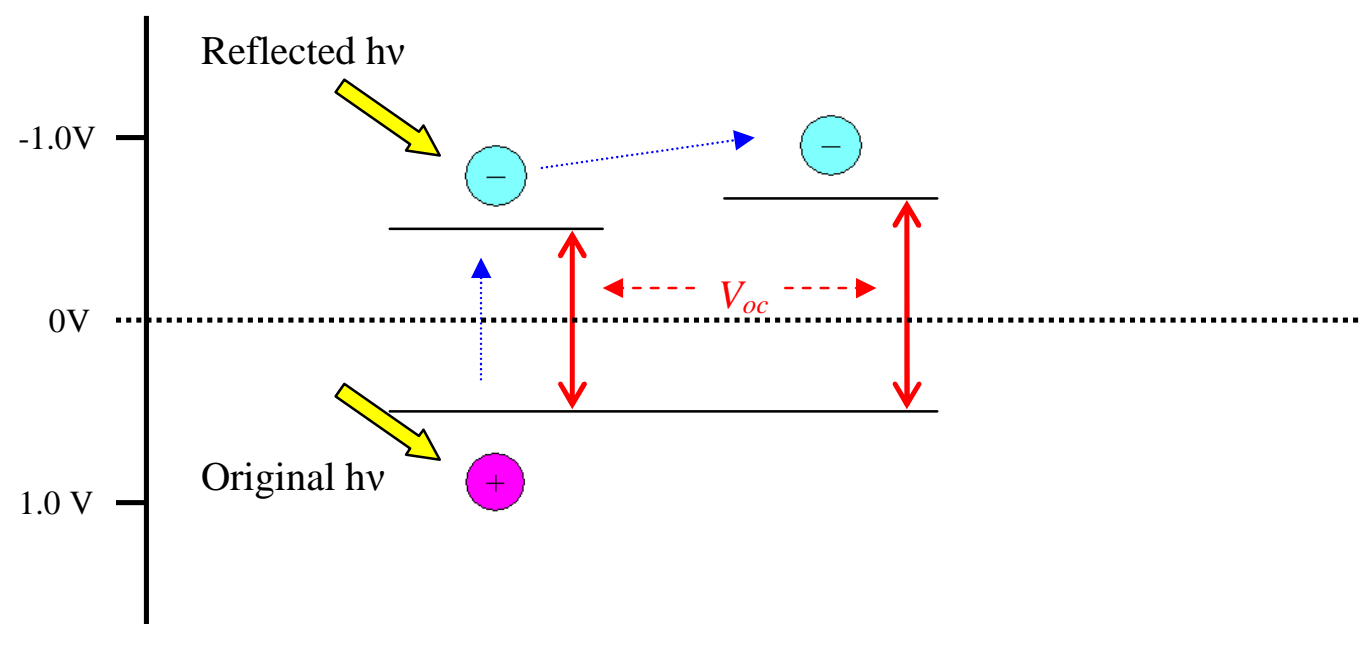

V vs. NHE

Figure 59. Speculated Reason Why the Open-Circuit Potential Appears to Increase Upon Back Reflection. 
The Teflon tape backing clearly increased the cell current, well beyond any experimental uncertainty (Figure 60(b)). This was confirmed by backing a normal gasket cell (of $0.10 \mathrm{~g} / \mathrm{cm}^{3}$ loading of RNCaSil), which gave an $I_{s c}$ of $18.3 \pm 2.2 \mu \mathrm{A} / \mathrm{cm}^{2}\left(352.4 \pm 44.2 \mu \mathrm{A} / \mathrm{cm}^{3}\right)$, a $45 \%$ increase over the equivalent un-backed cell.
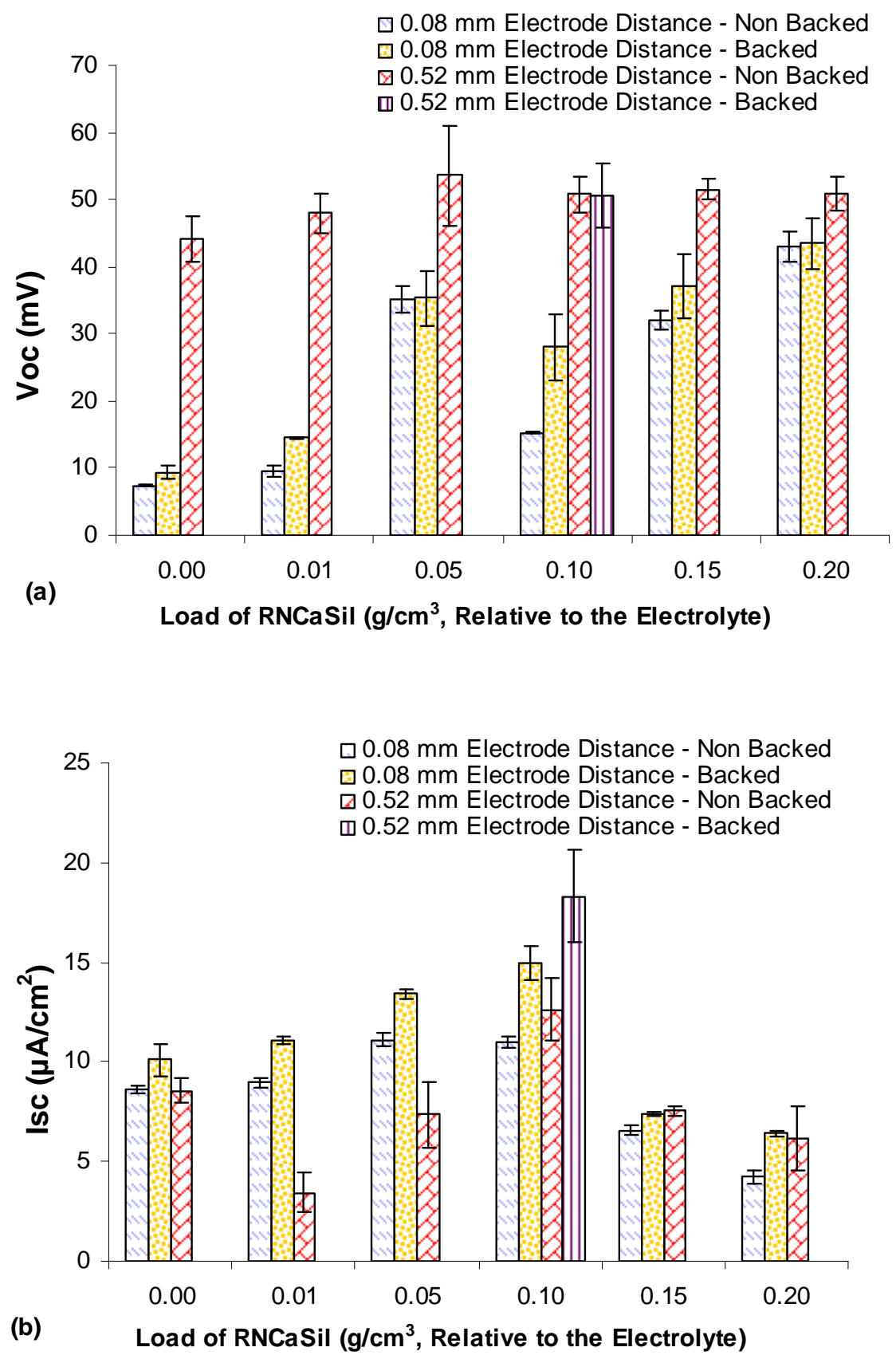

Figure 60. Effect of Cell Backing with RNCaSil in a Water Iodide/Triiodide Electrolyte ( 5 wt\% $\mathrm{CaCl}_{2}$ ); (a) $V_{o c}$ and (b) $I_{s c}$ in $\mu \mathrm{A} / \mathrm{cm}^{2}$. 


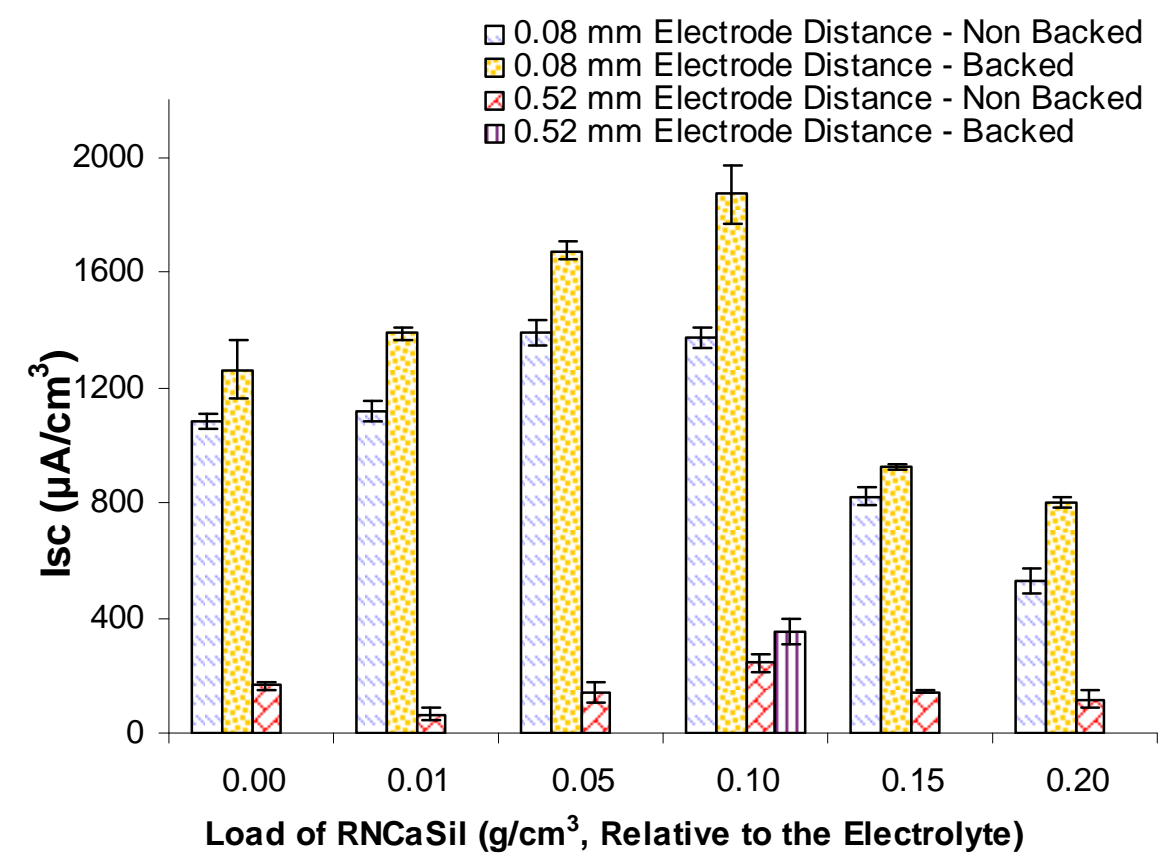

Figure 61. Effect of Cell Backing with RNCaSil in a Water Iodide/Triiodide Electrolyte (5 wt\% $\left.\mathrm{CaCl}_{2}\right)$; $I_{s c}$ in $\mu \mathrm{A} / \mathrm{cm}^{3}$.

The increase in $I_{s c}$ caused by the reflection could also explain the observed rise in $V_{o c}$. If there were a general rise in current, this could also increase the voltage (Figure 62).

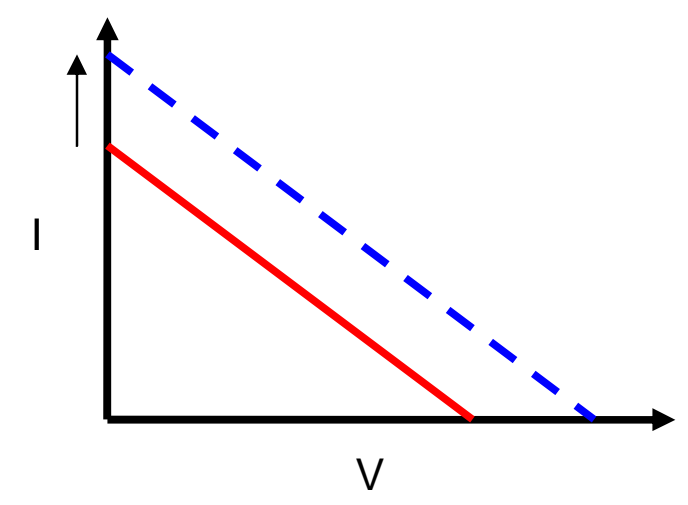

Figure 62. The Increase in Current Could Increase Corresponding Voltages.

Therefore, having light reflected back into the cell from behind the dark electrode increased, at least, the cell current. An enhancement of cell performance due to back-scattered light has also been observed with DSSCs. ${ }^{[132,133]}$ Hore et al. ${ }^{[133]}$ used a light scattering layer comprising of relatively large particles of $\mathrm{TiO}_{2}$ (rutile) or $\mathrm{ZrO}_{2}$, of 500 to $1000 \mathrm{~nm}$ in size, compared to the active $\mathrm{TiO}_{2}$ particles, which were 25 to $40 \mathrm{~nm}$. This scattering layer essentially "trapped" the light, which had been lost through the transparent cell. They found that the use of such a layer could increase current density by 
up to $80 \%$. Further, the use of the scattering layer meant that less active $\mathrm{TiO}_{2}$ had to be used to achieve the equivalent current. A similar effect was observed here. Where the current of the normal RNCaSil to electrolyte ratio used $\left(0.10 \mathrm{~g}\right.$ to $\left.1.0 \mathrm{~cm}^{3}\right)$ was lower for the $0.08 \mathrm{~mm}$ gasket than the normal gasket (Figure 60(b)), this was reversed when the $0.08 \mathrm{~mm}$ gasket cell was backed with Teflon tape. The effect could be enhanced by using multiple reflectors.

Generally, as the load of RNCaSil in the cells increased, the gain observed by backing the $0.08 \mathrm{~mm}$ gasket cells was diminished. The denser the cell contents was the less likely any light was going to reach the back of the cell and be reflected back in. 


\section{$7 \quad$ The Electrodes}

The electrodes used can greatly affect the overall cell performance and eventual marketability of a solar cell. The dark electrode is usually coated with gold, platinum or carbon (Part I 1.1.3) and previous experiments had shown the importance of the coating on the dark electrode. For example, long-term testing resulted in carbon being removed from the back electrode (Part IV 9.3), as did testing of electrode distance in the slotted-vessel (Part IV 5.2.3). Because of this, platinum was often used for coating the back electrode for experiments requiring more stability and for testing more acidic samples, such as the synthetic gels (Part IV 9.5.1). This first section of this chapter compares the use of platinum, carbon or gold for coating the back electrode to assess whether results from cells utilising different back-electrode coatings could be compared to each other.

As ITO glass is relatively expensive, the aim of the experiment discussed in section 2 of this chapter was to determine whether conductive carbon tape on "normal" (non-ITO-coated borosilicate) glass could act as the catalyst required for the iodide/triiodide process and be conductive enough to allow for a reasonable cell current. Following this, sections 3 and 4 discuss the potential use of a Pt-wire back electrode. Finally, section 5 examines the use of RNCaSil and iodide/triiodide in plastic electrodes (ITO-coated polyethylene terephthalate, ITO PET), for which there is much commercial value, due to the flexibility and potential ease of manufacture that plastic offers.

\subsection{Different ITO-glass Electrode Coatings}

RNCaSil was tested with (water-based) iodide/triiodide in normal gaskets with either Au-, Pt- or Ccoated back electrodes. The results obtained showed that the carbon and platinum coating were similarly effective, while the values achieved with gold were lower (Figure 63). It was visually observed that the gold coating was readily removed from the ITO glass, after only short-term, one-off tests. This was likely why the Au-coated electrodes were not as efficient and a large uncertainty with such cells was observed. Due to size restraints of the platinum coater, experiments were predominantly carried out with C-coated back electrodes, but where platinum was used rather than carbon, the data indicated that the results for cells composed of Pt- or C-coated back electrodes were inter-comparable. 

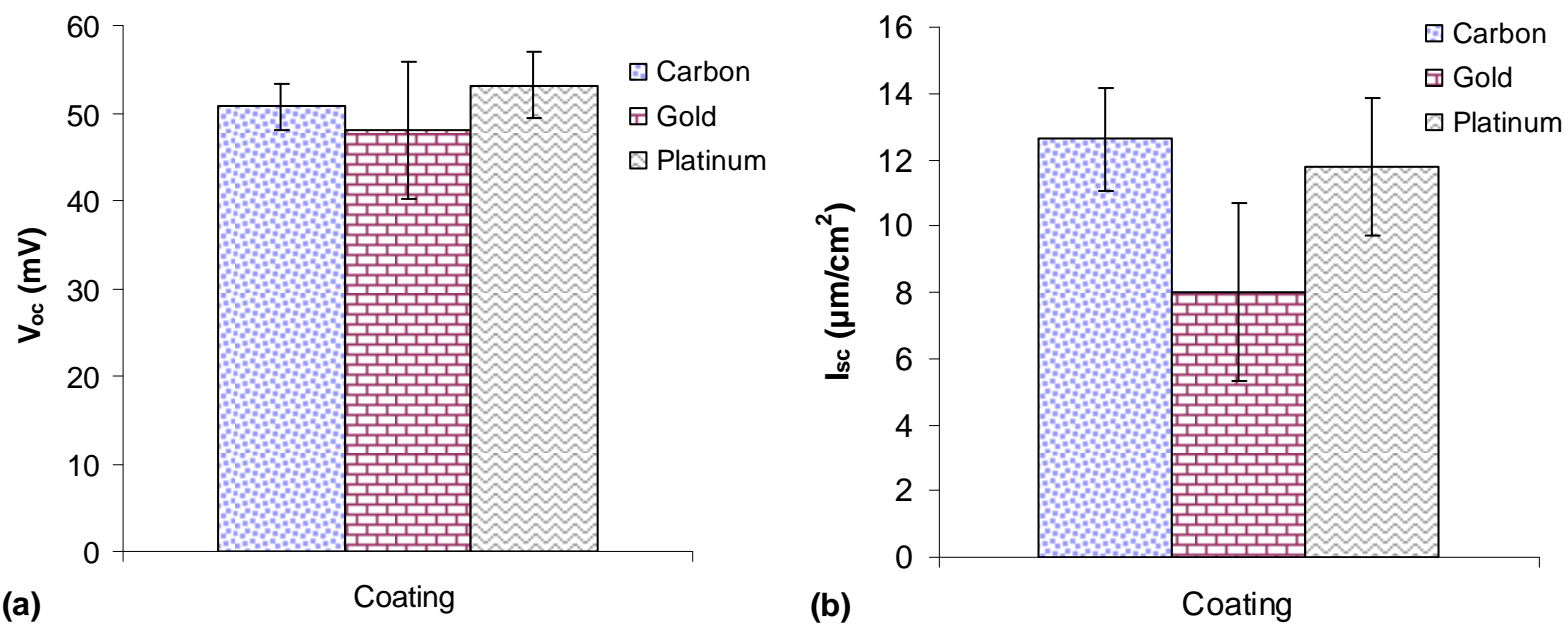

Figure 63. RNCaSil with Water-Based Iodide/Triiodide in Gasket Tests with Different Back-Electrode Coatings; (a) $V_{o c}$ and (b) $I_{s c}$.

\subsection{Carbon-Tape Coated "Normal" Glass}

Carbon tape on normal glass was used as the back electrode and ITO-coated glass as the photoelectrode. This was tested with RNCaSil and $70 \mathrm{wt} \%$ ethanol-based iodide/triiodide electrolyte. The experiment proved unsuccessful. Though able to achieve a reasonable potential, the tape was clearly not conductive enough, as very little current was observed (Figure 64).

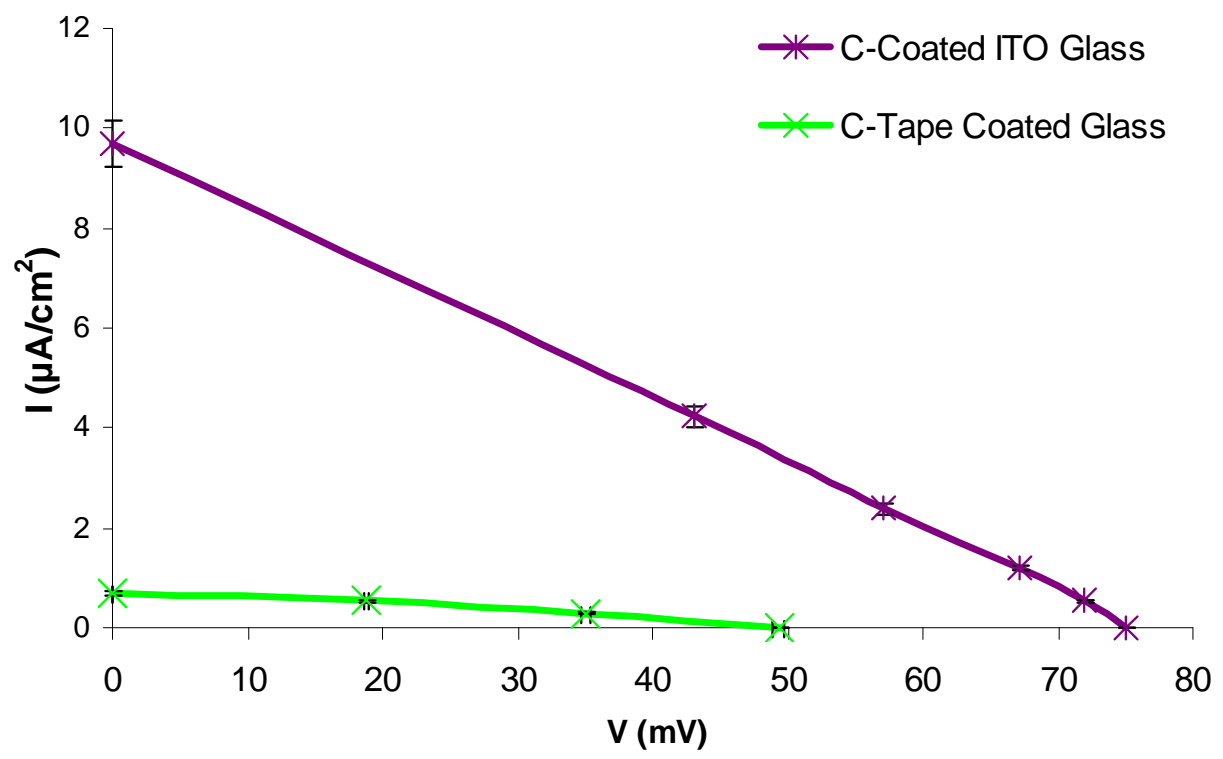

Figure 64. Current versus Voltage of RNCaSil in $70 \%$ Ethanol $30 \%$ Water Iodide/Triiodide: C-Tape Coated Normal Glass Compared to C-coated ITO Glass, Back Electrode. 


\subsection{Position of Electrodes}

The position and angle of the electrodes relative to the light was examined. This was achieved by studying a RNCaSil to iodide/triiodide ( $5 \mathrm{wt} \% \mathrm{CaCl}_{2}$ ) beaker cell, with the electrodes either parallel to the light source, or perpendicular to the lamp, either with the C-coated ITO electrode towards the back of the cell (as a normal dark electrode) or closest to the light (where the photoelectrode usually is) (above, Figure 15). This was repeated for three samples, each with a different distance between the electrodes $(0.5,2.0$ and $2.5 \mathrm{~cm})$.

The results for all three electrode distances were consistent and showed (as expected) that the optimal position of the electrodes was perpendicular to the light source with the C-coated ITO electrode being the back electrode (Figure 65). This data was interesting for two reasons. Firstly, because of how similar the results were for the parallel electrode arrangement and the electrodes with the C-coated ITO closest to the light. Secondly, there was an observable trend that, as the electrode distance widened, the gap between the performances of the different electrode positions increased. At an electrode distance of $0.5 \mathrm{~cm}$, there was no difference in cell potential, and the $I_{s c}$ from the cell with the ideal electrode positions was only approximately one-and-a-half times as great as those of the other two positions. However, as the electrode distance was increased, so did the divergence of cell performance observed as a consequence of the different electrode positions.

At 2.0 and $2.5 \mathrm{~cm}$, there was a marked difference observed between the potentials of the cells, depending on electrode position. This was also the case for the $I_{s c}$. Where, at $0.5 \mathrm{~cm}$, the difference in current was only around a factor of one-and-a-half, at electrode distances of 2.0 and then $2.5 \mathrm{~cm}$, this increased to a factor of three and then four. The diffusion of light was considered to be a possible reason for this observation. The shorter the distance between the electrodes, the more light that would have diffused through and reached the electrode furthest from the light source. Thus, with the $0.5 \mathrm{~cm}$ samples, it was arguable that one could have expected less of a variance between the two perpendicular samples. As one widened the gap between the two electrodes, the distance the light had to travel through the sample increased, and less light reached the back electrode. Hence, a greater difference was observed between when the light hit the uncoated-ITO electrode first and when it had to travel through the sample to reach the uncoated electrode. It was suspected that the parallel samples exhibited similar cell performance to the non-ideal perpendicular samples purely due to coincidence. In other words, that the decrease in cell performance caused by neither electrode facing the light was similar to the effect of the light having to travel through the sample to get to the uncoated-ITO electrode, was unrelated. 

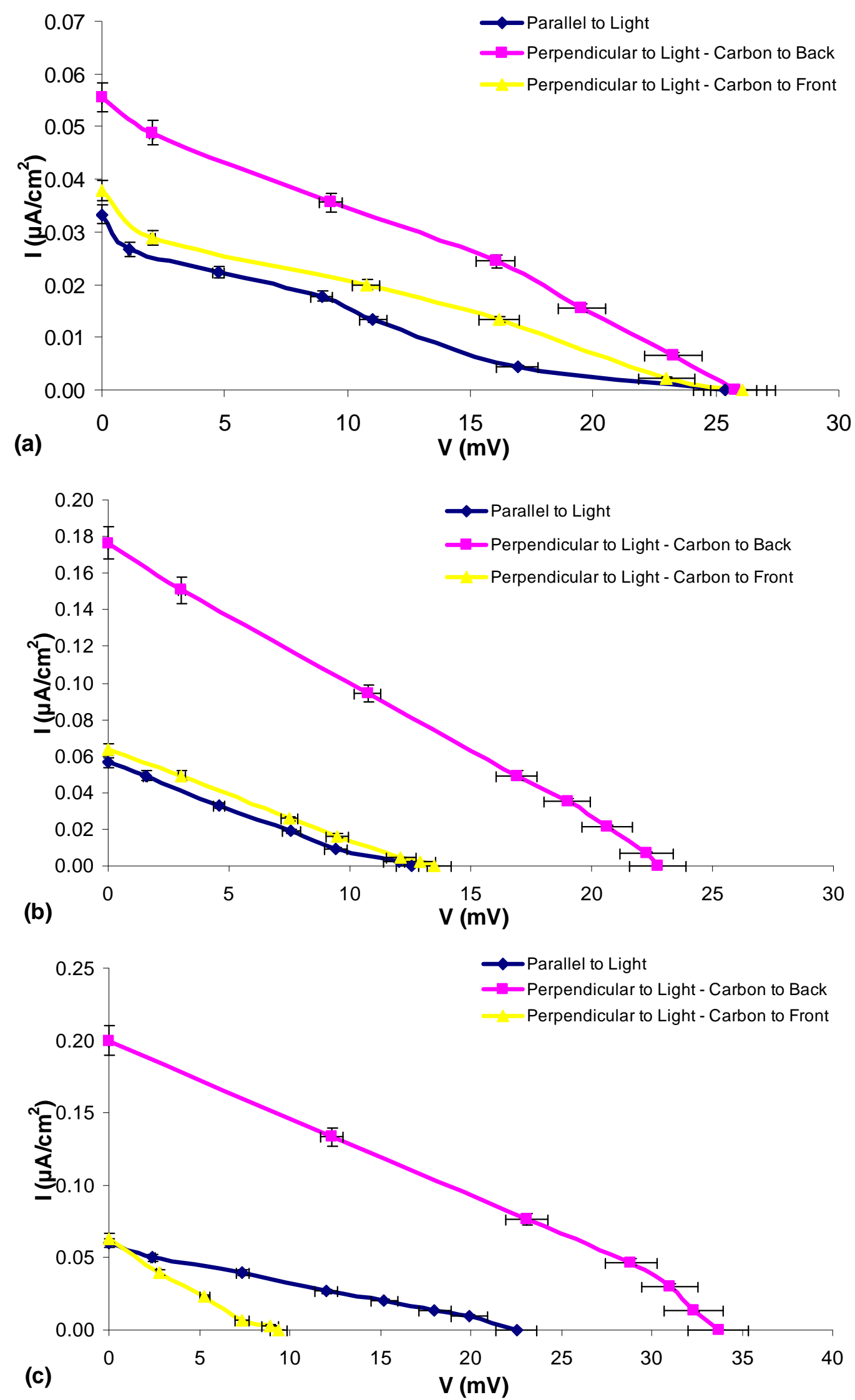

Figure 65. RNCaSil in Water Iodide/Triiodide Electrolyte $\left(5 \mathrm{wt} \% \mathbf{C a C l}_{2}\right)$. Beaker cells with Electrode Distances of: (a) $0.5 \mathrm{~cm}$; (b) $2.0 \mathrm{~cm}$; and (c) $2.5 \mathrm{~cm}$. 


\subsection{Testing in a Quartz Cuvette with a Pt-wire Electrode}

\subsection{1 $\quad \mathrm{I}_{2}$-EENCaSil Thin-Filmed Cell}

A thin film of $\mathrm{I}_{2}$-EENCaSil in $70 \mathrm{wt} \%$ ethanol iodide/triiodid was deposited onto the photoelectrode. A thin connection to the dark electrode was formed and the film allowed to dry overnight. The electrodes were arranged so that light shone onto the cell from between the two electrodes, in a beaker cell, without any filtration from the beaker. The performance of the cell was comparable to the gel cells (Figure 66, see Part IV 8). The higher cell performance was attributed to there being no filter between the light source and the active sides of the electrodes, i.e., the light below $295 \mathrm{~nm}$ was not filtered out by the beaker or the ITO glass.

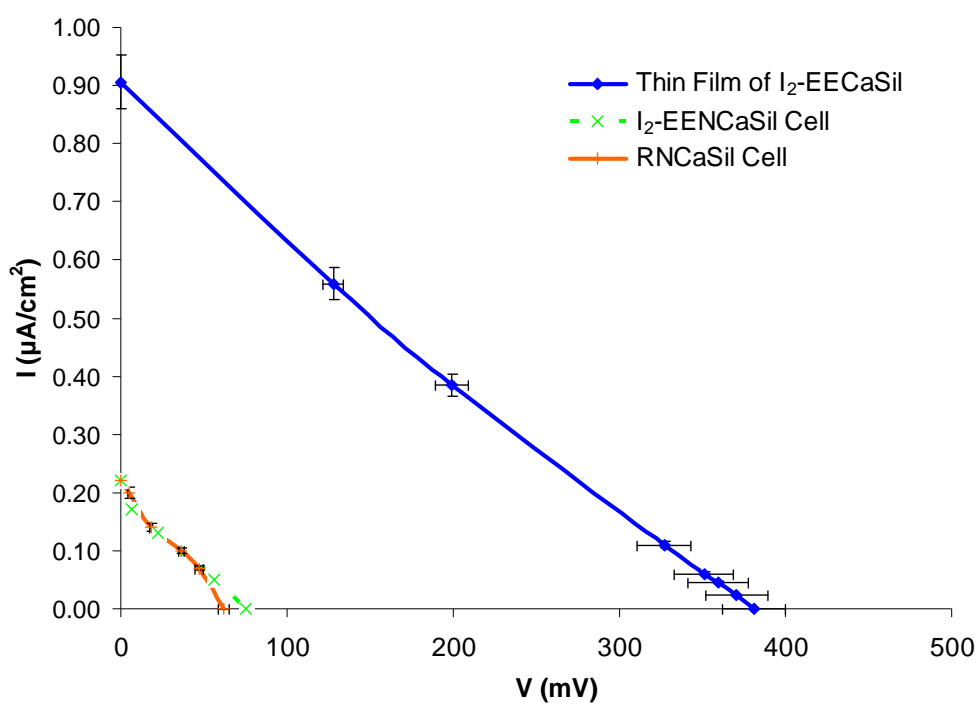

Figure 66. Current versus Voltage of $I_{2}$-RNCaSil Beaker Sample, Compared to the Equivalent Normal $I_{2}$-RNCaSil and RNCaSil Cells. All After 24 Hours Stirring in the Dark.

From this result, it was also thought that it was possible that the cells did not require a C-coated ITO glass back electrode and a Pt-wire electrode could suffice. The use of sheets or grids of platinum as the dark electrode is common with photovoltaics.

\subsubsection{Pt-wire Electrodes}

To determine whether one of the ITO-glass electrodes normally used could be replaced by Pt-wire, tests with RNCaSil and $70 \mathrm{wt} \%$ ethanol $30 \%$ water iodide/triiodide were performed in a quartz cuvette. The Pt-wire was tested both as the dark electrode, with an ITO-glass photoelectrode, and as the photoelectrode, with a Pt-coated ITO-glass dark electrode. For the latter sample, a sheet of ITO glass 
was also placed in front of the cell to filter out light that would not have passed through in the former cell (Experimental, Part III 7). As a comparison, the use of two ITO-glass electrodes (as were normally used) was also tested in the quartz cuvette.

Given that, even with DSSCs, the back electrode is usually either coated glass or a platinum sheet, it was expected that, of the Pt-wire cells, the most efficient would be that with the wire as the dark electrode (Figure 67). It was of interest that both the $V_{o c}$ and $I_{s c}$ of the said cell were greater than the quartz cell with two ITO-glass electrodes. This was attributed to the higher conductance of Pt-wire than ITO glass. When Pt-wire was used as the photoelectrode, lower $V_{o c}$ and $I_{s c}$ were achieved, which was not surprising. The cell performance of this cell was enhanced slightly when an ITO glass sheet was placed in front of the cell. However, this was only minor and attributable to experimental uncertainty.
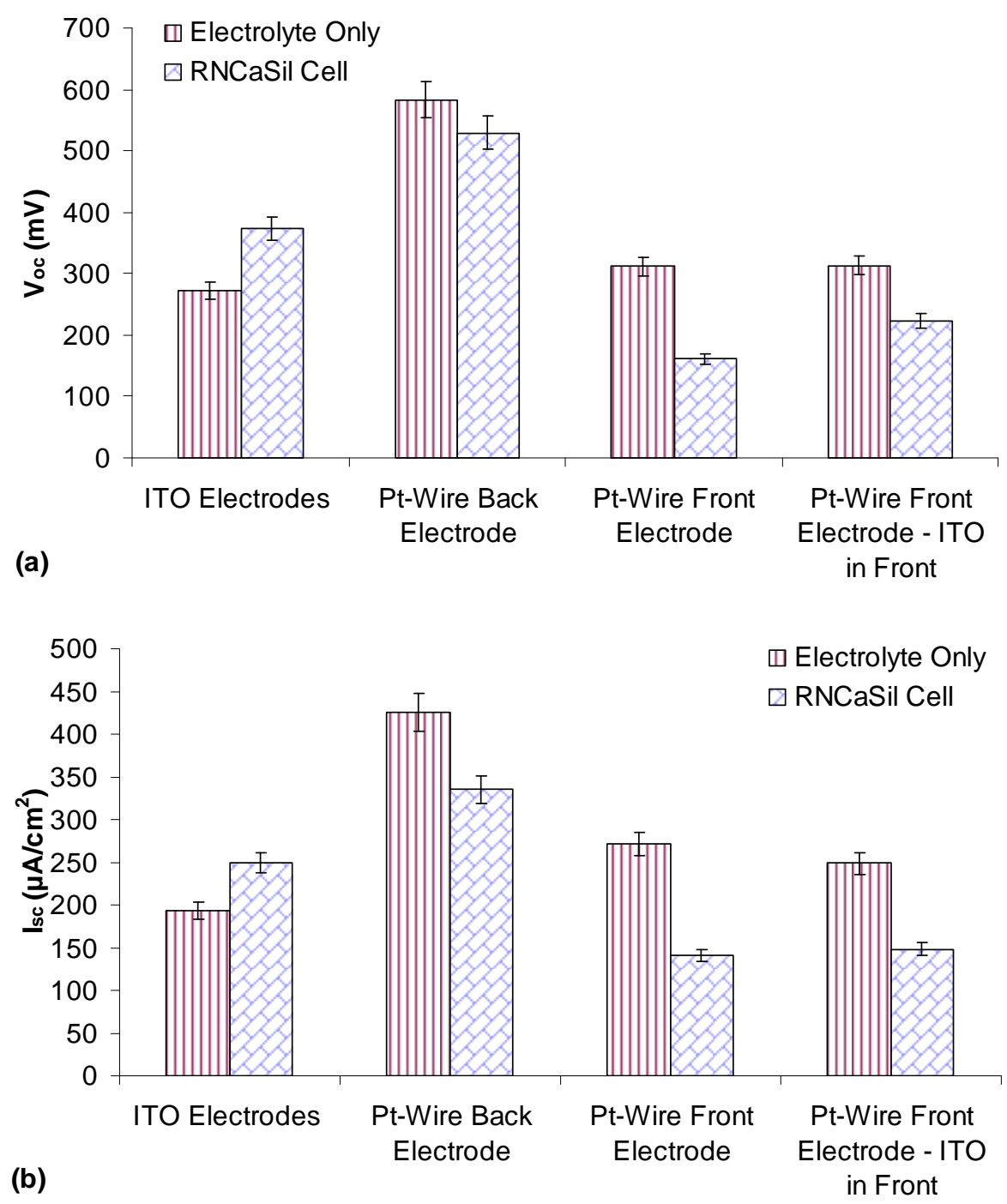

Figure 67. RNCaSil with $70 \%$ Ethanol $30 \%$ Water Iodide/Triiodide Electrolyte in a Quartz Cuvette, with Different Electrode Combinations; (a) $V_{o c}$ and (b) $I_{s c}$. 
What was particularly interesting was that all the quartz cuvette cells, whether using the standard two ITO-glass electrodes or Pt-wire, showed higher currents and potentials than achieved in any other cell, including equivalent gasket cells. The best performing quartz cell was that with the Pt-wire as the dark electrode and the electrolyte alone, which gave a power conversion efficiency of $2.6 \%$. This was far greater than an equivalent gasket cell $\left(5.6 \times 10^{-3} \%\right)$. Furthermore, the $I_{s c}$ and $V_{o c}$ were over sixty and eight fold, respectively, that of the same sample in a gasket cell. Compared to the best g-RNCaSil cell gasket cell (dried and tested with the water iodide/triiodide, discussed below, Part IV 9.1), the Pt-wire back-electrode cell with RNCaSil had a power conversion efficiency over forty four times greater (0.6 $\%$ compared to $13.3 \times 10^{-3} \%$ ) and the $I_{s c}$ and $V_{o c}$ were thirteen and ten times higher, respectively.

Even the less efficient RNCaSil-containing quartz cell, with two ITO-glass electrodes, exhibited a power conversion efficiency of $49.2 \times 10^{-3} \%$, which was over five times larger than the comparative gasket cell $\left(8.5 \times 10^{-3} \%\right.$, the $I_{s c}$ and $V_{o c}$ were twenty seven and five times higher, respectively).

These differences were not due to the cuvettes being made of quartz glass, as with ITO glass (as purchased from Sigma Aldrich) the ITO is coated onto normal glass, not quartz glass. This was confirmed by UV-vis (Figure 68). Consequently, all light of wavelengths lower than $295 \mathrm{~nm}$ were blocked by the use of ITO glass as a photoelectrode. Furthermore, the only cell without ITO glass filtering the light source achieved the lowest results.

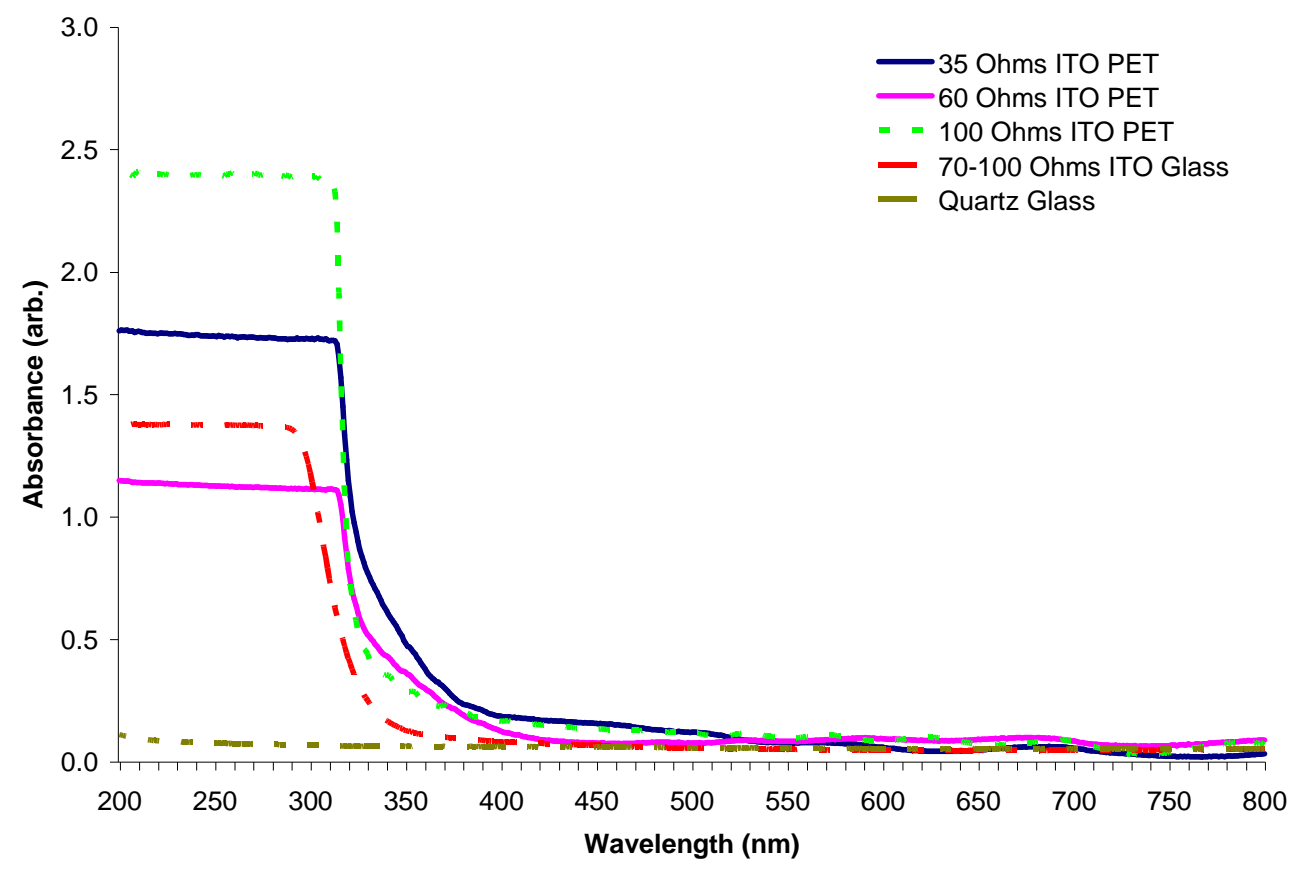

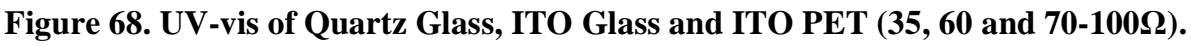


The iodide/triiodide couple has been known to be corrosive towards platinum electrodes. ${ }^{[3]}$ Thus, it was arguable that the obtained values were due to the Pt-wire interacting with the iodide/triiodide, beyond the expected catalysis. This could explain why the iodide/triiodide electrolyte alone acted more favourably with the Pt-wire than when with RNCaSil, which could have hindered the process. It also explained why it was only when there were two ITO-glass electrodes that the RNCaSil cells worked better than the electrolyte alone. However, this did not explain why the cells with the two ITO-glass electrodes work so well compared to gasket cells. Additionally, if the iodide/triiodide couple were so prone to interacting with platinum, one would have expected to see such interaction, at least to some minor degree, when Pt-coated ITO glass was utilised as the dark electrode in gasket cells. However, Ptcoated ITO glass exhibited similar cell performances as C-coated ITO glass and greater stability.

As already stated, the conductivity of the Pt-wire is higher than that of ITO glass, however this did not explain why the cells with the two ITO-glass electrodes worked so well, compared to gasket cells.

NCaSils have been observed to be deactivated when hit directly with intense light, such as by the research lamp through quartz glass. This accounted for why the RNCaSil-containing cell showed a lower current and potential than the electrolyte alone, when Pt-wire was used as the photoelectrode. However, it did not rationalise why the problem was not rectified by placing a sheet of ITO glass in front of the cell. Furthermore, it did not elucidate why the electrolyte also performed better when the Pt-wire was the back electrode.

None of these contemplations remotely clarified why the quartz cells generally performed so much more efficiently than gasket cells. It was not believed that it was related to cell depth. Testing in the slotted vessel (Part IV 5.2.3) showed that there was nothing particular about a cell depth of approximately $1 \mathrm{~cm}$. Thus, it remained a mystery.

\subsection{ITO-coated Plastic Electrodes}

There is a lot of value in having plastic electrodes (Part I 1.1.4). The main problem with plastic electrodes in Graetzel type DSSCs is that they cannot withstand the temperatures required to sinter the $\mathrm{TiO}_{2}$. However, as there were issues with sintering NCaSils, it was predicted that, if the cells were behaving as DSSCs, the cell performance would not be degraded in plastic electrodes, as compared to 
when in ITO-glass electrodes. Consequently, tests were carried out in ITO-coated polyethylene terephthalate (ITO PET). Other cell parameters remained unchanged from those of normal gasket cells, except that Pt-coating was used rather than C-coating for the back electrodes.

It was clear that the RNCaSil and iodide/triiodide sample did not function as well in ITO PET as in ITO glass (Figure 69). Currents in the ITO-PET electrodes were less than one third of those from equivalent ITO-glass cells. That the currents from the ITO-PET cells were similar, regardless of the different resistances of the plastics, indicated that the limiting factor was not the conductance of the sheets, but was related to the RNCaSil sample and its inability to function in combination with the ITOPET electrodes.
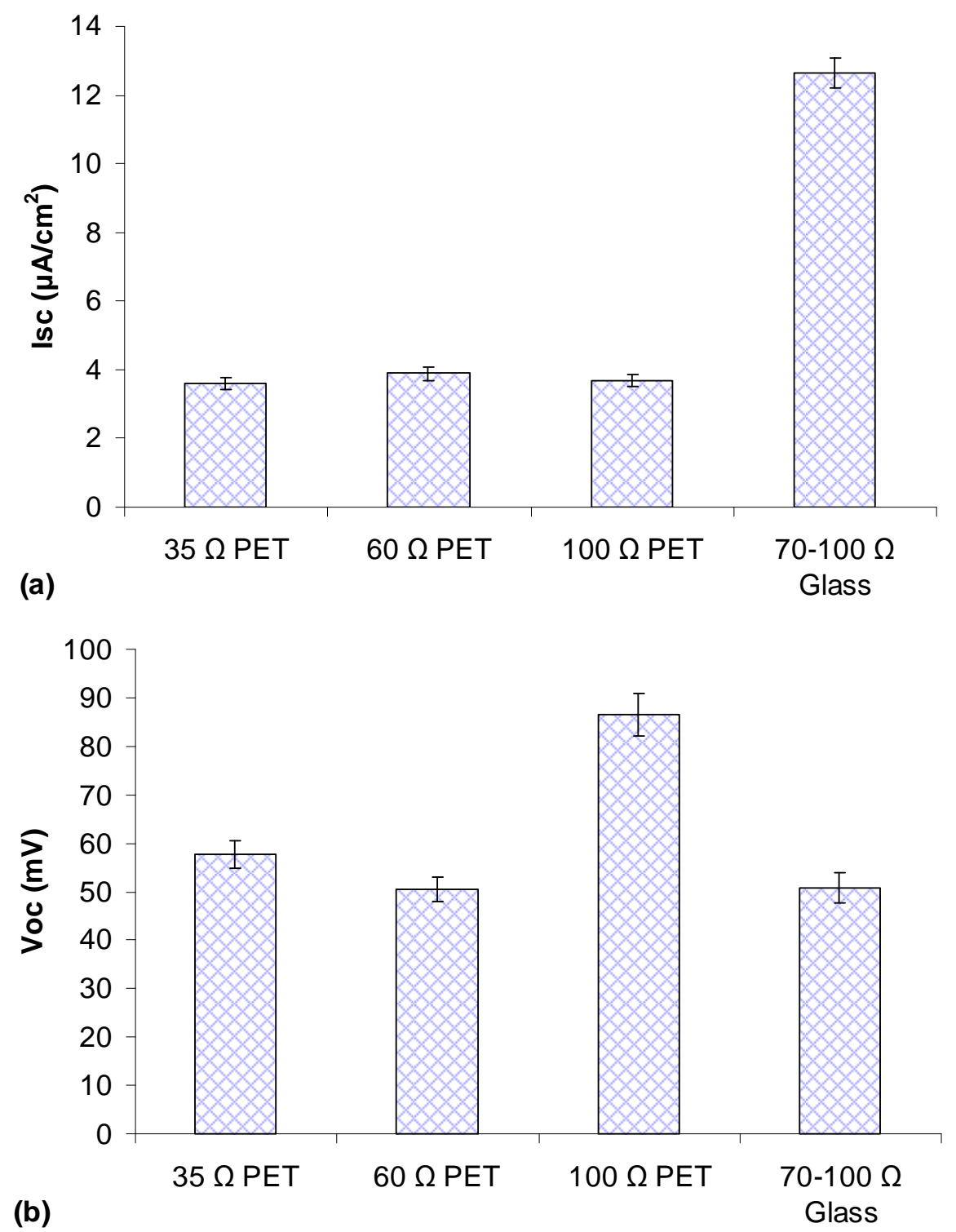

Figure 69. RNCaSil in Water Iodide/Triiodide Electrolyte $\left(5 \mathrm{wt} \% \mathrm{CaCl}_{2}\right)$ in Different Electrodes; (a) $I_{s c}$ and $(\mathrm{b}) V_{o c}$. 
This was attributed to poorer connectivity of the cell sample with the ITO-PET electrodes than ITOglass electrodes. When ITO-PET cells were constructed, the samples were not compressed into the gasket sample area the way that they were with ITO-glass cells. This was due to the flexibility of the PET. When the cells were constructed, they could not be pressed together over the sample area, which allowed the PET over the sample area to flex outwards rather than push down on the sample, resulting in poor sample-to-electrode connectivity (Figure 70). This problem did not arise with non-flexible ITO glass. Therefore, the use of ITO glass throughout this project was valid and sufficient.

(a)
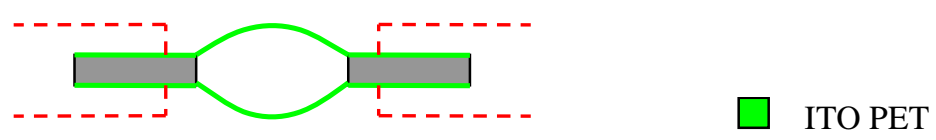

$\square$ Clips

$\square$ Teflon Gasket

(b)

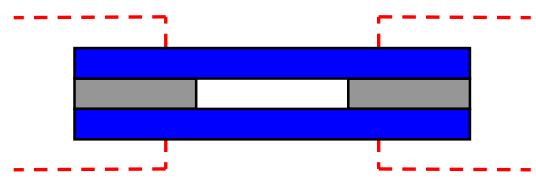

ITO Glass

Figure 70. Side-On Representation of (a) ITO PET; and (b) ITO-Glass Cells.

The $V_{o c}$ did not appear to be suppressed in the ITO PET, which was attributed to the fact that a cell with poor connectivity could still achieve the same potential difference with whatever connectivity was achieved. Why the potential obtained with the $100 \Omega$ ITO PET was so much higher than the other three samples was not known. It was suspected that it was a spike in the data, which often occured with NCaSil cells.

It is prudent to note that, though lower efficiencies were obtained in ITO PET, some loss in cell performance could be considered acceptable, given the benefits gained by using plastic rather than glass. Glass is heavy and lacks flexibility. ${ }^{[15]}$ Conversely, plastic is light, able to be rolled up, cut to any desired shape and could be manufactured by a continuous roll-process. ${ }^{[15]}$ However, the loss of $69 \%$ current here seemed quite a large sacrifice.

\subsubsection{Lamination}

Because of the thinness and flexibility of plastic electrodes, it was thought that it would be possible to laminate cells comprised of ITO-PET electrodes. As far as known, lamination of plastic electrode cells 
had not been reported, though the possibility had been realised. ${ }^{[29]}$ The cells were constructed in the same fashion as normal gasket cells, except that $100 \Omega$ ITO PET was used instead of ITO glass, and non-plastic-coated $\mathrm{Sn}$ - or $\mathrm{Cu}$-wires were used as the contacts.

A cell comprising of tetrabutylammonium hexafluorophosphate with RNCaSil, KI, iodine and water, which had proven to work reasonably well in ITO-glass electrodes (Part IV 4.3.4), exhibited almost no activity when placed in a laminated cell with tin contacts. This was unexpected, as though samples had shown lowered performances in ITO PET than ITO glass, such a large loss of performances was surprising.

Such an unanticipated decreased performance was also exhibited by a gel NCaSil cell (g-NCaSil, Figure 71). This gel was formed by leaving $1.0 \mathrm{~g}$ of RNCaSil and $10 \mathrm{~cm}^{3}$ of $70 \mathrm{wt} \%$ ethanol, iodide/triiodide electrolyte to develop for 5 days (gel formation is discussed in the following chapter). The inactivity of these cells aroused suspicions that: (1) the tin contacts were not conductive enough; (2) the lamination process dried out the cells, such that they were no longer functional; (3) the laminated plastic filtered out much more light; or (4) the laminated cells did not put enough pressure on the sample area, meaning that the contact between the electrodes and the samples was not sufficient.

\subsubsection{Tin or Copper Contacts}

That the use of tin contacts was insufficient was proven by putting the same aforementioned gel ( 5 days later) into identical ITO-PET cells, except that one had tin contacts while the other had copper contacts. It was expected that the cell performances would be decreased somewhat, as, unless under illumination, gel performance decreased over time. Un-laminated, the cell with tin-contact's cell performance was virtually non-existent (Figure 71). Conversely, the cell with copper contacts exhibited an efficiency greater than the original laminated g-NCaSil cell with tin contacts.

The lowered cell performance was not attributable to different conductivities. Copper has a high conductivity of $58.4 \mathrm{MS} / \mathrm{m}\left(25^{\circ} \mathrm{C}\right),{ }^{[127]}$ which was limited by the lower conductivity of the indium used to connect the contact wires to the electrodes, which is only $12 \mathrm{MS} / \mathrm{m}\left(25^{\circ} \mathrm{C}\right) .{ }^{[127]}$ The tin contacts used had an even lower conductivity of $8.7 \mathrm{MS} / \mathrm{m}\left(25^{\circ} \mathrm{C}\right) .{ }^{[127]}$ However, the truly limiting conductivity was that of the ITO Glass at 0.25 to $0.43 \mathrm{MS} / \mathrm{m}^{[134]}$ Therefore, the poor performances were attributed to poor contact between the tin and ITO, likely caused by an oxide layer on the tin. 


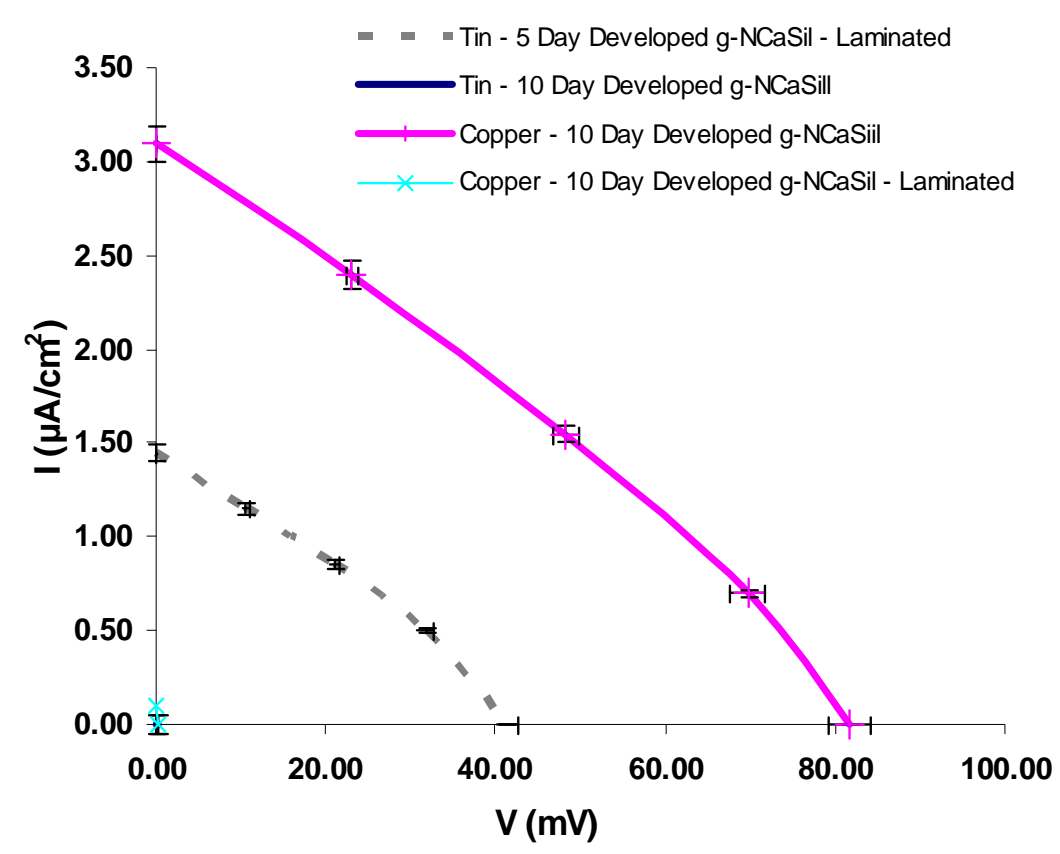

Figure 71. Current over Voltage Plots of g-NCaSil in ITO-PET cells; with Tin or Copper Contacts, after 5 or 10 Days of Gel Formation, Laminated or Not.

Furthermore, the results also implied that the lamination process somehow limited cell performance. To assess the implications of laminating ITO-PET cells, the same g-NCaSil cell with copper contacts was laminated and tested under the research lamp. This caused the cell performance to become negligible (Figure 71). This showed that the lamination process did decrease cell performance and also indicated that, had the original cell with tin contacts not been laminated, its efficiency would have been much greater, and even more so had copper contacts been used instead of tin. Thus, the decreased cell performance was also related to the lamination process.

\subsubsection{Solvent Evaporation Caused by Lamination}

There must have been evaporation of a small amount of sample during the lamination process, at approximately $75^{\circ} \mathrm{C}$. However, the speed and method of the procedure would have limited this. Furthermore, the cells did not look dry, but retained their gel-like appearance. The small amount of solvent loss did not explain the disproportionate reduction in cell efficiency.

\subsubsection{The Laminated Plastic}

UV-vis absorption was performned on the laminated plastic used to laminate the cells. This showed that, though this plastic filtered out lights of wavelengths lower than $300 \mathrm{~nm}$ to a greater extent than the 
ITO PET used, it did not inhibit any wavelengths not filtered out by the PET (Figure 72, see also the transmission spectra, Appendix, Part VII 2, Figure 101). Hence, it was not thought that the plastic used to laminate the cells was insufficiently transparent. The ITO PET was the limiting factor in this respect.

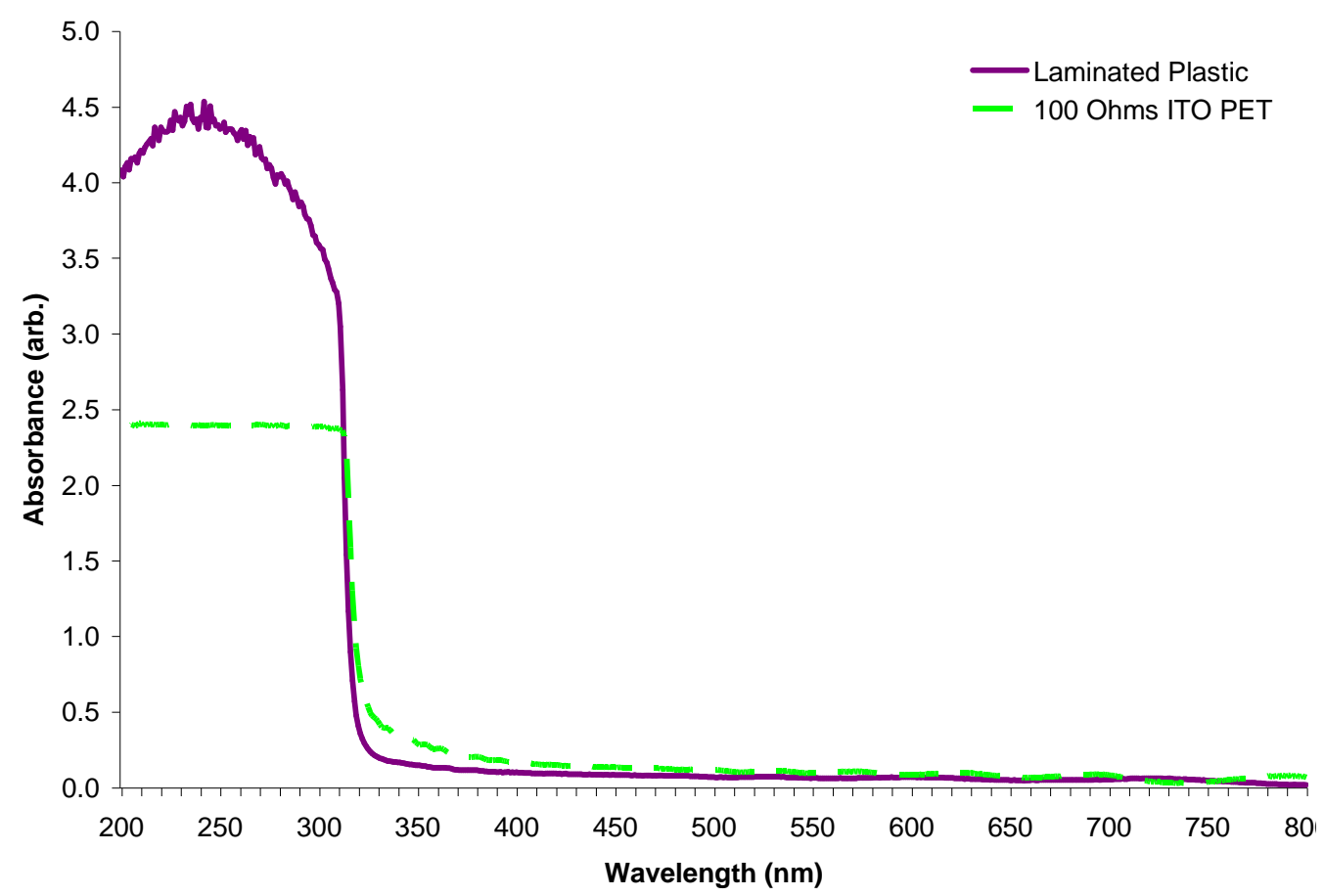

Figure 72. UV-vis of the Laminated Plastic Used to Laminate the Cell Compared to the ITO PET Used.

It was unlikely that the ITO PET infused into the cell samples during lamination, as the process took place at approximately $75^{\circ} \mathrm{C}$, a temperature too low to degrade the PET. ${ }^{[15]}$

\subsubsection{Sample and ITO PET Contact}

As the above two rationales hypothesised, for decreasing cell performances on lamination, were dismissed, the effect was credited to inadequate contact between the sample and the plastic electrodes. Indeed, this was visually observable. Unlike ITO glass, the ITO PET was flexible and bulged slightly outwards (as discussed above, Part IV 7.5), rather than lying perfectly flat and pushing against the sample. There were parts of the cell area where one did not observe the the sample flush against the ITO PET. One way to overcome this, in future, is to use a greater amount of a semi-solid or solid sample than the sample volume of the gasket. This would force the sample to sit against the electrodes.

The flexibility of the PET also made it was difficult to laminate NCaSil powder/liquid electrolyte cells. Due to the liquid nature of the sample, it often did not stay in the sample area during lamination. 


\section{$8 \quad$ Long-Term Testing and Gel Formation}

For a solar cell to have any real application, it must be stable over long periods of time. This chapter discusses the longer-term testing of NCaSils, over several days, in the iodide/triiodide electrolyte, prepared in different solvents. Furthermore, it discusses the formation of gel cells from such long-term testing. These investigations were carried out in beaker cells, as gasket cells were difficult to seal off and tended to dry out over time. Currents obtained in the beaker tests were much lower than those from gasket tests of the same samples. This was because of the curvature of the beaker, a larger surface area (see above, Part IV 5.1) and there being sample between the light source and photoanode (see below, Part IV 9.3). Thus, results from beaker cells could only be compared to those of other beaker cells.

\subsection{Solvent Effect}

Samples of RNCaSil in differently solvated iodide/triiodide electrolytes were left stirring in the dark for 72 hours, except during testing after certain periods of time, after which they were illuminated continuously for a further 72 hours. The phase prior to continuous illumination has already been discussed in some depth earlier (Part IV 4.4.2.1) and, as such, this section focuses on cells illuminated after the 72 hour dark period.

It could be seen (Figure 73) that most samples improved in cell performance over time, both when left in the dark stirring and particularly when illuminated. This effect was observed in both the potential and the current, but particularly the latter, with the exception of the sample in $70 \mathrm{wt} \%$ ethylene glycol iodide/triiodide, which exhibited an improvement in $V_{o c}$. The ethylene glycol sample was by far the most unstable and was the only sample where the $I_{s c}$ decreased on constant illumination. This was attributed to the higher viscosity of ethylene glycol, which made the system require a longer period of time to reach equilibrium. Furthermore, by the end of the 72 hour illumination period, the sample was stark white (Figure 74). It was thought that the reason why the $V_{o c}$ of this sample continued to climb, yet the $I_{s c}$ remained low, was related to this complete photobleaching. The near complete formation of iodide over triiodide created a large potential difference, but removed the photoactive species, eliminating most of the current. 


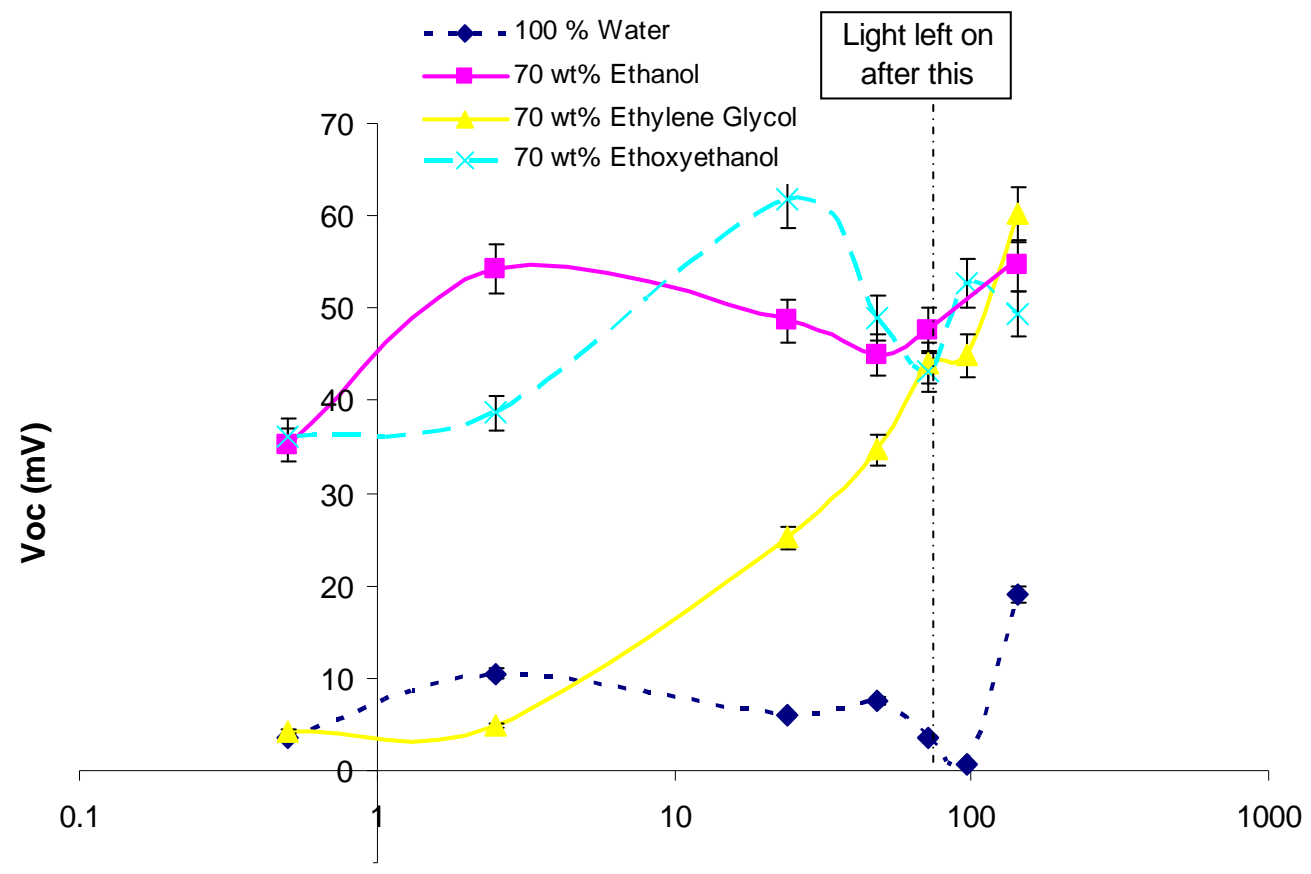

(a)

Time (hr)

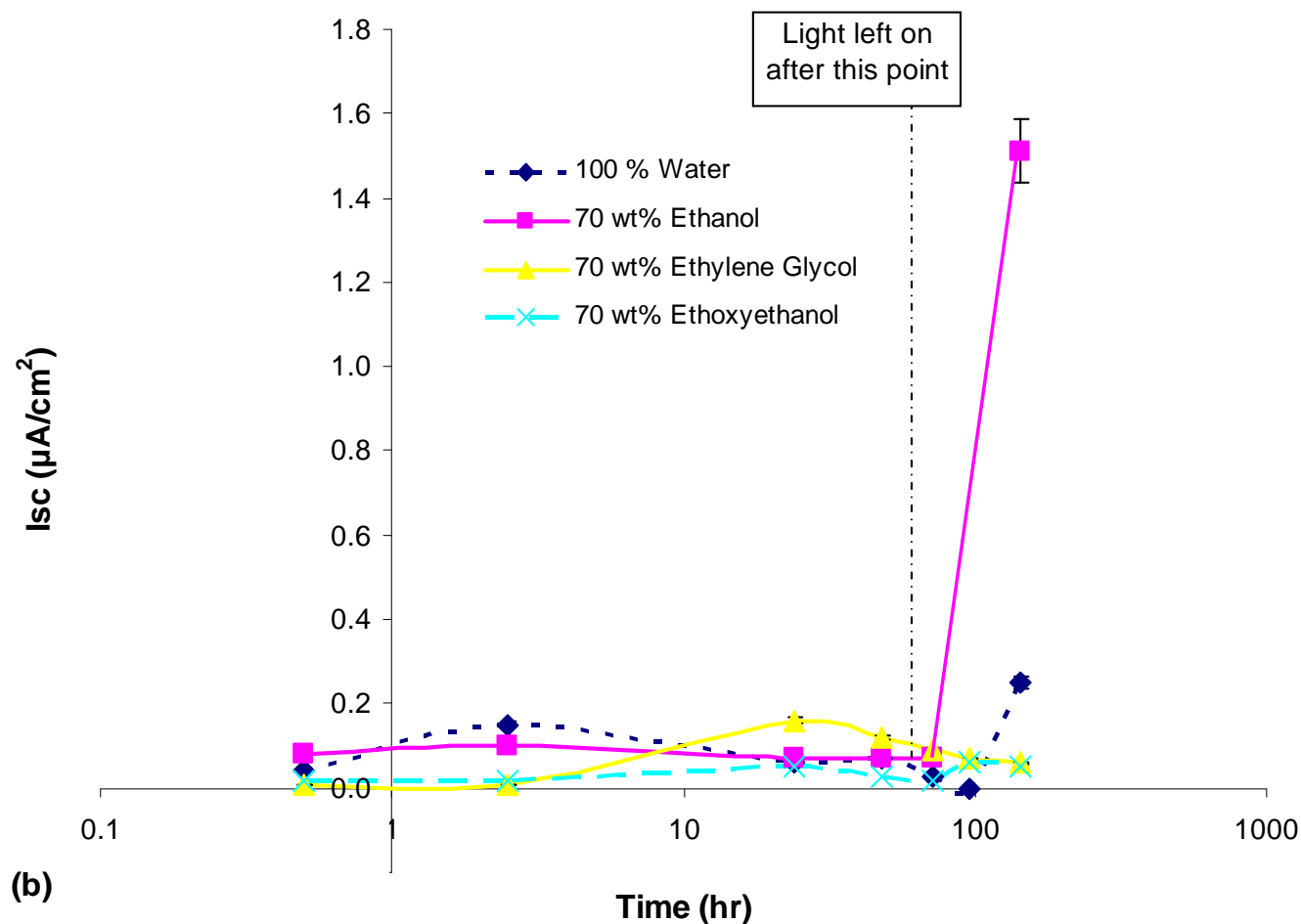

Figure 73. Long-Term Testing of RNCaSil in $30 \mathrm{wt} \%$ Water $70 \mathrm{wt} \%$ Other Solvent Iodide/Triiodide Electrolyte; (a) $V_{o c}$ and $(\mathbf{b}) I_{s c}$.

Why ethoxyethanol made a poor solvent to RNCaSils has already been discussed (Part IV 4.4.2.1), and was attributed to the fact that it was readily absorbed onto NCaSils blocking its pores. Thus, it was not 
surprising that it showed little improvement on constant illumination. The cell performance of the water-solvated electrolyte also improved on constant illumination, but this was incomparable to that observed with the $70 \mathrm{wt} \%$ ethanol sample.

All the samples showed instability; the currents and voltages were inconsistent between each reading, with the exception of the $70 \mathrm{wt} \%$ ethanol sample, which was comparatively stable. This changed after the sample was illuminated continuously for 72 hours. Afterwhich, the $V_{o c}$ increased and the $I_{s c}$ multiplied by over twenty one fold. The reason for this rapid rise in current was the fact that the sample had turned into a single-phased gel, g-NCaSil (Figure 74). The $70 \mathrm{wt} \%$ ethanol-based sample was the only one that did so. Each of the other samples maintained clearly separated solid and liquid phases.

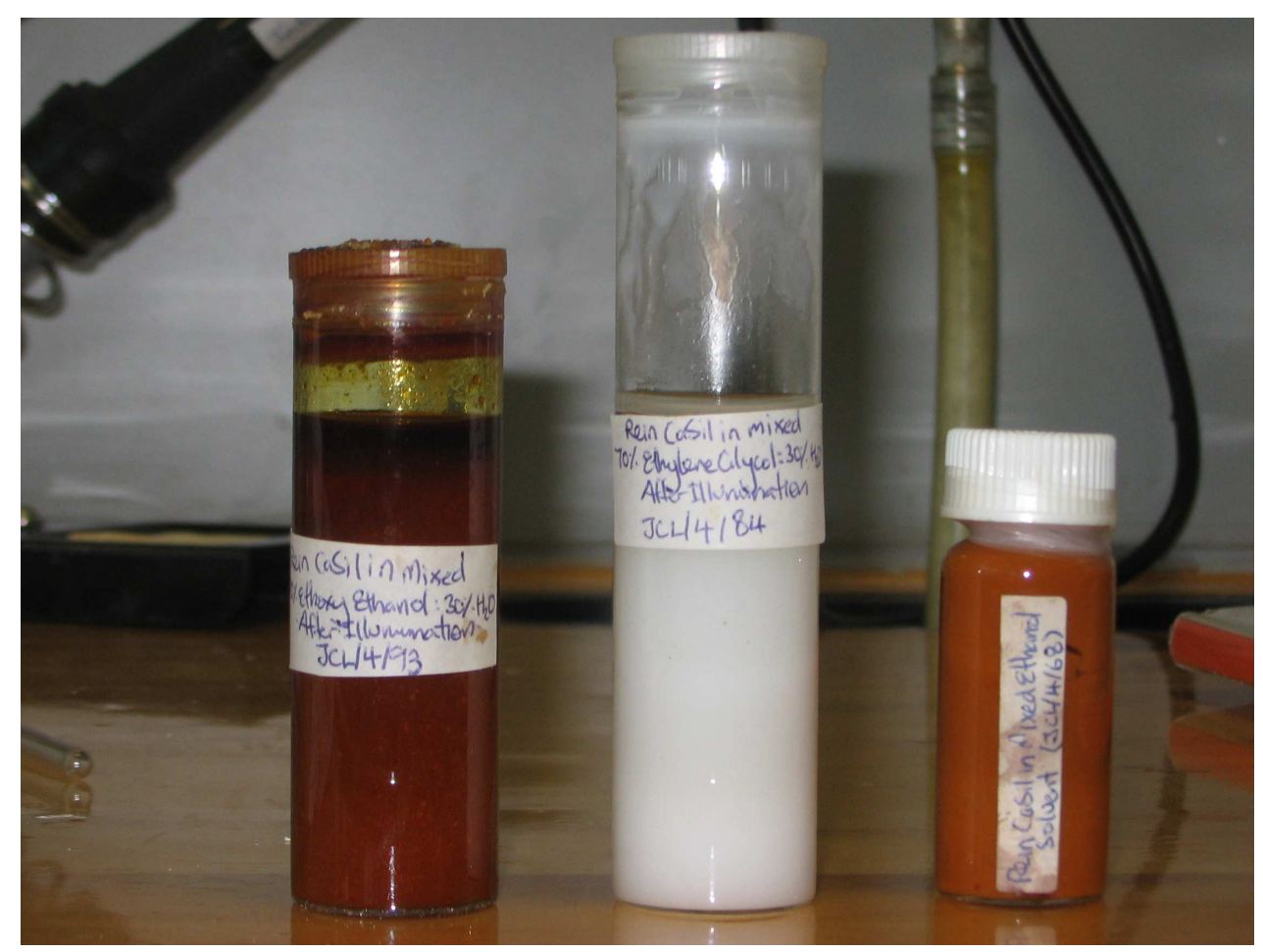

Figure 74. 72 Hours Illumination of RNCaSil in 70 wt\% Ethoxy Ethanol (Left), Ethylene Glycol (Centre) and Ethanol (Right) Iodide/Triiodide Electrolytes.

\subsection{Repeatability of Forming g-NCaSil}

\subsubsection{The RNCaSil}

An initial attempt to repeat the formation of $\mathrm{g}$-NCaSil was successful, but subsequent attempts were not. Initially, it was believed that this was due to a new batch of RNCaSil being used (Batch \#4); the 
previous batch used (Batch \#1) having been used up. Tests performed in gasket cells seemed to support this (Part IV 1.3.1). When differences in batches were resolved, and testing in gaskets indicated a RNCaSil (Batch \#5) consistent with the original RNCaSil used, this was utilised in a beaker test with $70 \mathrm{wt} \%$ ethanol iodide/triiodide. However, though results were initially promising, there was no gel formation and the current peaked at a point lower than that achieved by the g-NCaSils. Thus, the inconsistency of the RNCaSil was not the only issue. As is discussed in the following paragraphs, nor was the level of impurities in the ethanol determinative. Rather, gel formation depended upon solvent evaporation, the rate of which appeared important.

\subsubsection{Purity of the Ethanol}

As the ethanol used in the electrolyte of the first two g-NCaSils had come from the ends of a drum, it was thought that the gels may have arisen as a consequence of an impurity contained in the drum. The failure to form a gel and achieve greater currents from the absolute ethanol sample initially supported this hypothesis. Common impurities in drum ethanol are small amounts of acetic acid and other alcohols. ${ }^{[135]}$ Samples were prepared with absolute ethanol with 0.1 or $1.0 \mathrm{~mol} \%$ acetic acid or 1.0 mol\% butanol. Each of these proved unsuccessful, failing to form gels or achieve significant increases in currents. Thus, the purity of the ethanol was not determinative of gel formation.

\subsubsection{Evaporation}

It was also suspected that perhaps the samples required evaporation of some solvent. Beaker cells were sealed off with parafilm. It was thought that perhaps the g-NCaSil samples had not been fully sealed. Thus, a sample was prepared with the consistent RNCaSil Batch \#5, absolute ethanol (without any additives) and the beaker's parafilm cover was left partially unsealed. A gel was created, which achieved a good current, over $2 \mu \mathrm{A} / \mathrm{cm}^{2}$, comparable to that achieved by cells made from RNCaSil Batch \#1. Therefore, for the formation of g-NCaSil, there needed to be some solvent evaporation. It was possible that gel formation occurred because of an influx of oxygen into the sample from the air, however this did not seem likely as the presence of oxygen would not have assisted the iodide/triiodide's photogalvanic process nor the interaction between the silicate and iodine. Furthermore, X-ray photoelectron spectroscopy performed on the sample showed no such relationship (discussed below, Part IV 9.6). 
The same gel formation or cell efficiencies could not be achieved merely by changing the initial RNCaSil and electrolyte solution ratios, i.e., by using less electrolyte solution to begin with. Though a less-liquid sample was obtained, no gel-formation occurred. It appeared that time was required for gNCaSil to form. In other words, the rate of evaporation was important. This was supported by later analysis performed on the gel, which showed that the RNCaSil structure had been modified completely during gel formation (below, Part IV 9.4 and 9.6).

Though the initial three gel samples were all formed under constant illumination of the research arc lamp, later gels were made merely by leaving the samples on a bench, with access to air and ambient light. The g-V-NCaSil and g-Ce-NCaSil (discussed below, Part IV 9.2) were made as such. Though the time required was greater (168 to 240 hours, compared to 24 to 48 hours under the resarch arc lamp), such formation was arguably more convenient, as the research arc lamp could be used for other experiments, larger samples could be prepared and energy was not expended from the xenon arc lamp.

\subsection{Long-Term Testing of the Electrolyte Alone}

Importantly, the $70 \mathrm{wt} \%$ ethanol iodide/triiodide electrolyte alone (without any form of NCaSil added) did not exhibit such great increases in current (and obviously did not form a gel) on constant illumination (Figure 75). Though the $I_{s c}$ did rise, as was common on constant illumination, the values achieved were minimal compared to those of the g-NCaSils after the same period of illumination.
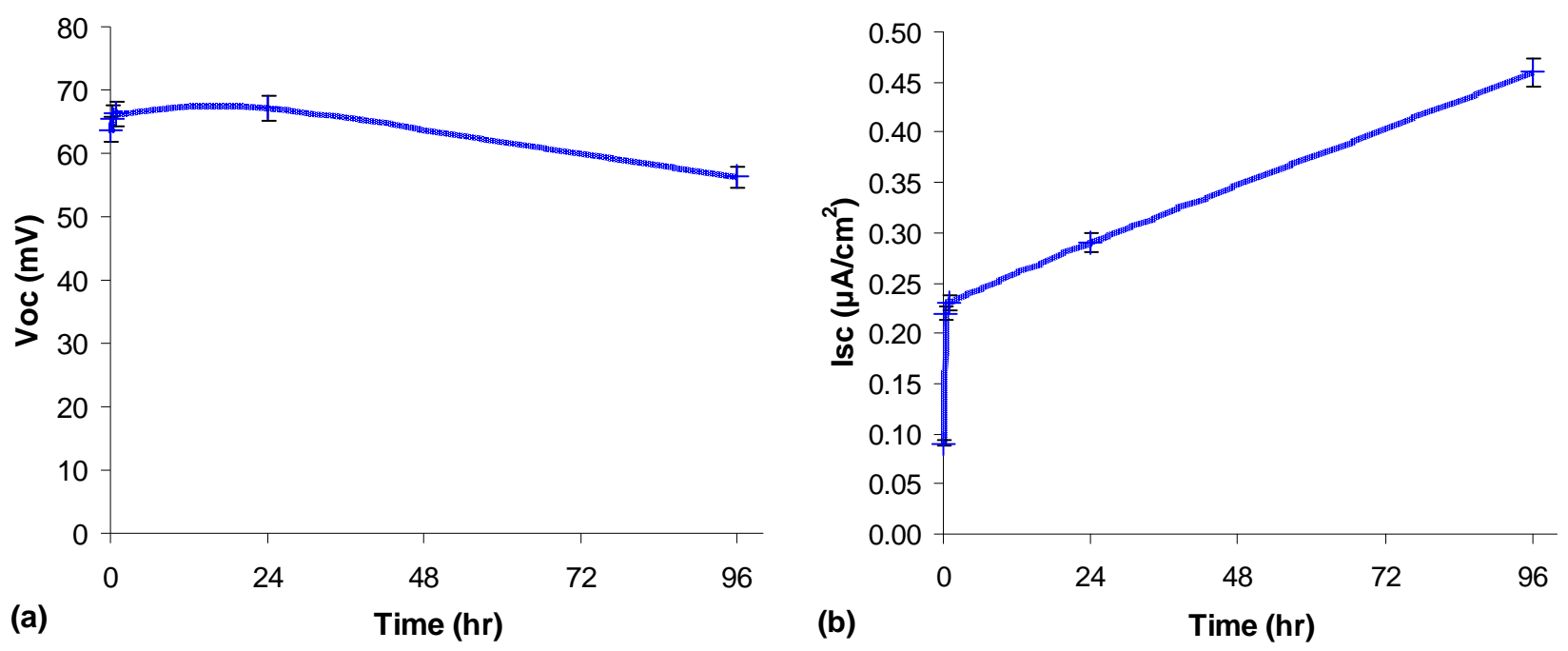

Figure 75. Constant Illumination of the $70 \mathrm{wt} \%$ Ethanol Iodide/Triiodide Electrolyte; (a) $V_{o c}$ and (b) $I_{s c}$. 


\subsection{Requirement of 72 Hour Settling Time}

All the aforementioned samples in this chapter were left stirring in the dark for 72 hours before constant illumination began. A sample was made up to ascertain whether the full 72 hours was required to achieve the same results on constant illumination. The sample was stirred in the dark for only 24 hours, before being continuously illuminated.

Though the RNCaSil used (Batch \#4) was not one found capable of forming a gel, these results indicated that whether the sample was stirred in the dark for 24 or 72 hours did not affect its cell performance on constant illumination (Figure 76). This conclusion was supported by the fact the gNCaSils formed from samples sitting on the bench, free of any stirring or intense illumination.
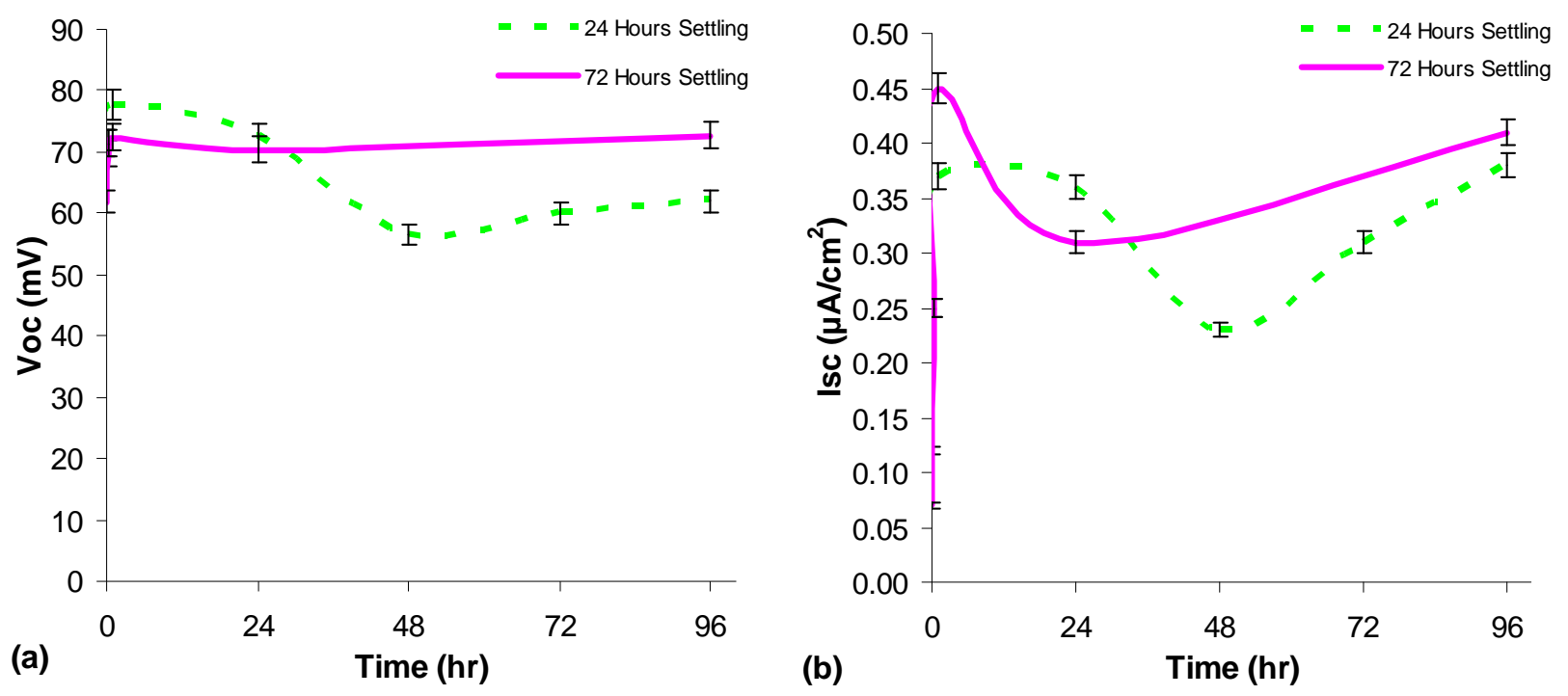

Figure 76. Constant Illumination of RNCaSil (Batch \#4) in 70 wt\% Ethanol Iodide/Triiodide Electrolyte, After 24 or 72 Hours Settling Time; (a) $V_{o c}$ and (b) $I_{s c}$.

Results from the illumination of $\mathrm{I}_{2}$-EENCaSil after either 24 or 72 hours of stirring in the dark differed because the $\mathrm{I}_{2}$ could be lost from the silicate structure over time, particularly with stirring, which was likely why 72 hours of stirring gave an initially high current, which dropped off over time (Figure 77). However, the sample seemed particularly prone to photobleaching, which likely assisted the rapid decline in current after 24 hours of illumination, as discussed below (Part IV 8.5).

It is important to note that, though the time required for gel formation varied depending on the form of NCaSil, whether a sample was stirred in the dark for 24 or 72 hours affected how long a sample had to 
be illuminated before it formed a gel. For example: whereas the $\mathrm{I}_{2}$-EENCaSil sample given a 72 hour settling time resulted in gel formation after 72 hours illumination, the sample given only 24 hours settling time had not. The latter sample took somewhere between 96 and 120 hours of illumination to form a gel.
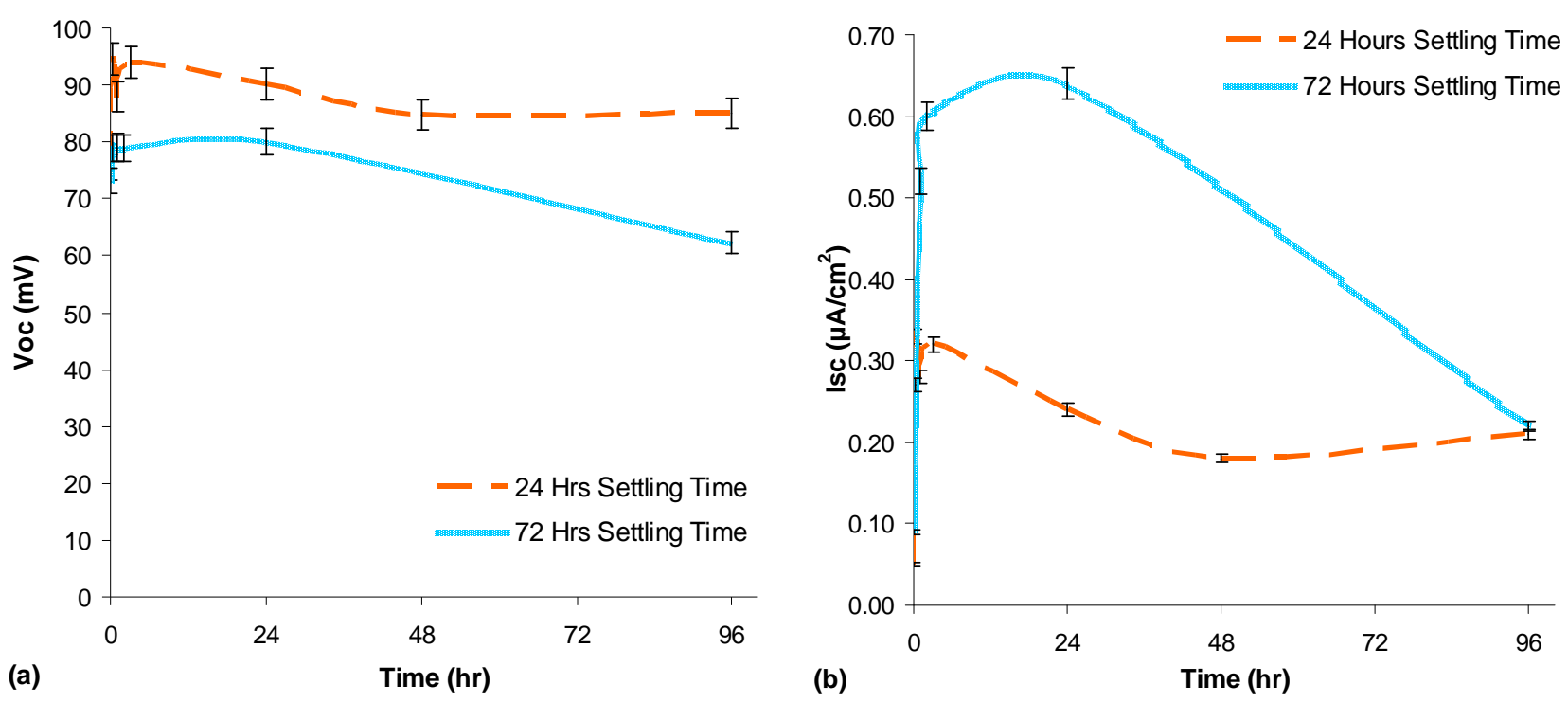

Figure 77. Constant Illumination of $\mathbf{I}_{2}$-EENCaSil in $70 \mathrm{wt} \%$ Ethanol Iodide/Triiodide. After 24 and 72 Hours Settling; (a) $V_{o c}$ and (b) $I_{s c}$.

\subsection{Different Forms of NCaSils in $70 \mathrm{wt} \%$ Ethanol Iodide/Triiodide}

Many different NCaSil forms were tested in $70 \mathrm{wt} \%$ ethanol iodide/triiodide electrolyte to determine which of the NCaSils were capable of forming gels. All samples were left for 72 hours stirring in the dark before being illuminated constantly. Unreinforced NCaSil, EENCaSil and AWNCaSil were all unable to form gels the way that RNCaSil did. The backbone structures of these other NCaSils were unable to be maintained long-term in solution, whether in water or ethanol, and simply broke apart. Comparatively, the RNCaSil was able to form gels, due to its greater stability, which allowed for the silicate backbone to be modified (see below, Part IV 9.4) rather than be completely lost.

Although the initial results for Ru-EENCaSil seemed promising, the sample did not become a gel until after 96 hours illumination, by which time both the potential and current had decreased significantly. Thus, Ru-EENCaSil showed no long-term stability. The decline in the $V_{o c}$ and $I_{s c}$ was attributed to the loss of Ru from the silicate structure over time. 
$\mathrm{I}_{2}$-EENCaSil became a gel after approximately 72 hours. However, after 96 hours, it was relatively dry, and almost completely photobleached. This drying and photobleaching also eventually occurred for the sample stirred in the dark for only 24 hours. While the sample between electrodes remained unbleached, but the surrounding sample was almost completely white. It was thought that the reasons why the performance declined after around 24 hours were the loss of iodine from the silicate structure over time, quick-drying of the sample and photobleaching.

The loss of ruthenium and iodine from the NCaSils and subsequent loss of cell performance was not indicative of the cell type produced. Whether the NCaSil were acting as semiconducting materials or as insulating materials for soggy sand electrolytes, one would have expected cell performance to decrease upon the disconnection of the additives from the NCaSil backbones.

The V-and Ce-NCaSil samples were illuminated for 24 hours, afterwhich they were left sitting on a bench, whereupon gel formation occurred (after 288 hours). As is discussed below (Part IV 9.2), these gels proved quite active. Hence, $\mathrm{V}$ - and $\mathrm{Ce}-\mathrm{NCaSil}$ obviously required more time than RNCaSil to become gels and reach their peak activity. This was possibly due to them being derived from unreinforced NCaSil. 


\section{$9 \quad$ Performance and Analysis of NCaSil Gels}

As discussed in the preceding chapter, over the course of 24 to 48 hours of illumination, RNCaSil formed a gel when used in conjunction with a $70 \mathrm{wt} \%$ ethanol $30 \mathrm{wt} \%$ water based iodide/triiodide electrolyte. In this chapter, the performance of the formed gels in normal gasket cells is discussed, and the gels analysed by NMR, X-ray photoelectron spectroscopy (XPS) and UV-vis. Attempts at synthetically forming the gels are also presented.

\subsection{Cell Performance of g-NCaSil}

g-NCaSil proved to be more efficient in a photovoltaic setup than RNCaSil powder/electrolyte cells. The gel could be used both in its original semi-liquid state alone and when dried into a powder form (suspected to be an aerogel), used in conjunction with an electrolyte. In both forms, even 14 days after its formation, the current observed for a cell employing g-NCaSil was double that of the equivalent RNCaSil powder/electrolyte cells for the semi-liquid state (Figure 78(a)) and for the aerogel state (Figure 79(a)), while the potentials were comparable for the semi-liquid state (Figure 78(b)) and for the aerogel state (Figure 79(b)). The overall cell efficiences were $11.6 \times 10^{-3} \%$ for the semi-liquid state and $13.3 \times 10^{-3} \%$ for the dried aerogel.
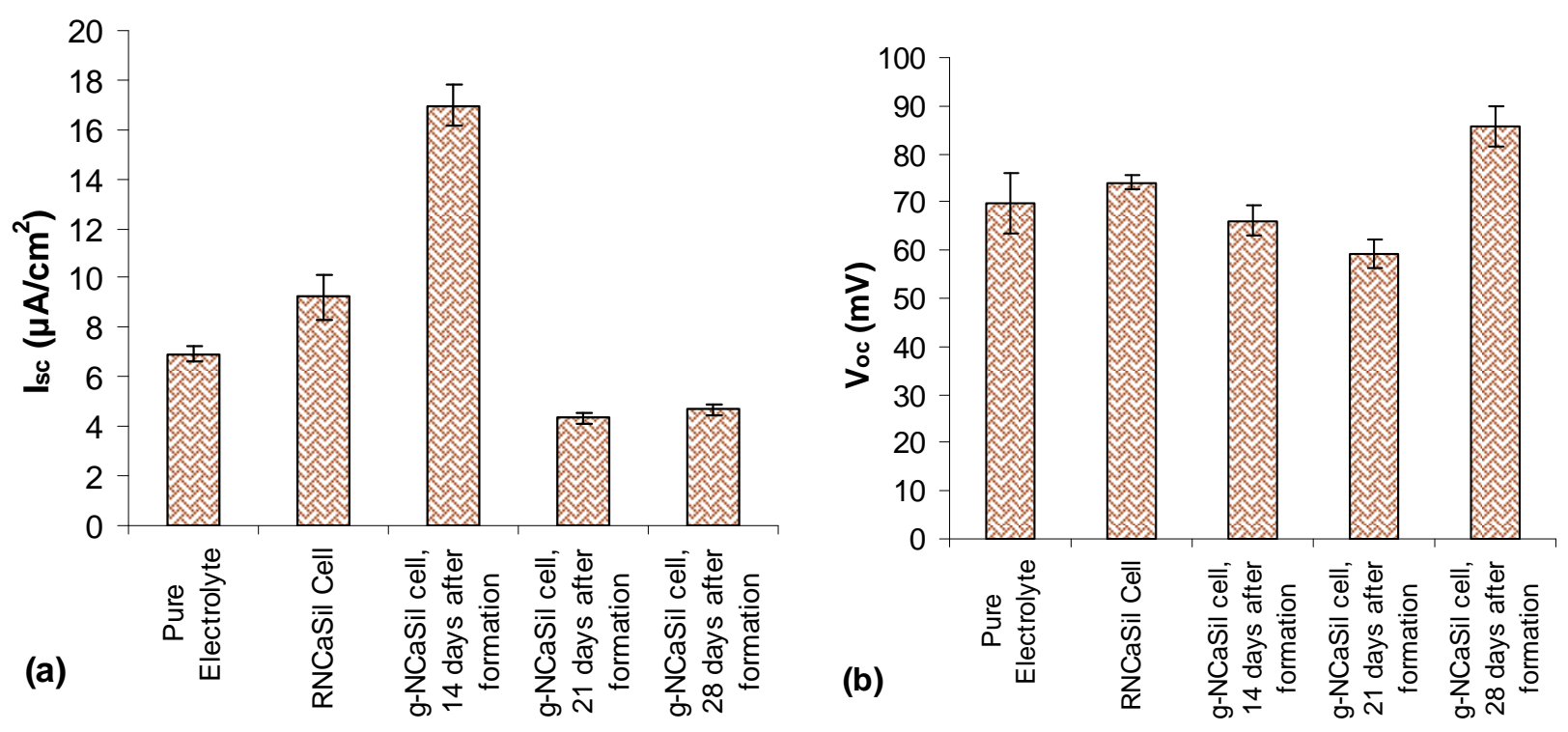

Figure 78. g-NCaSil 14, 21 and 28 Days After Formation, Compared to a 70 wt\% Ethanol 30 wt\% Water Iodide/Triiodide Electrolyte and an Equivalent RNCaSil-Based Cell; (a) $I_{s c}$ and (b) $V_{o c}$. 

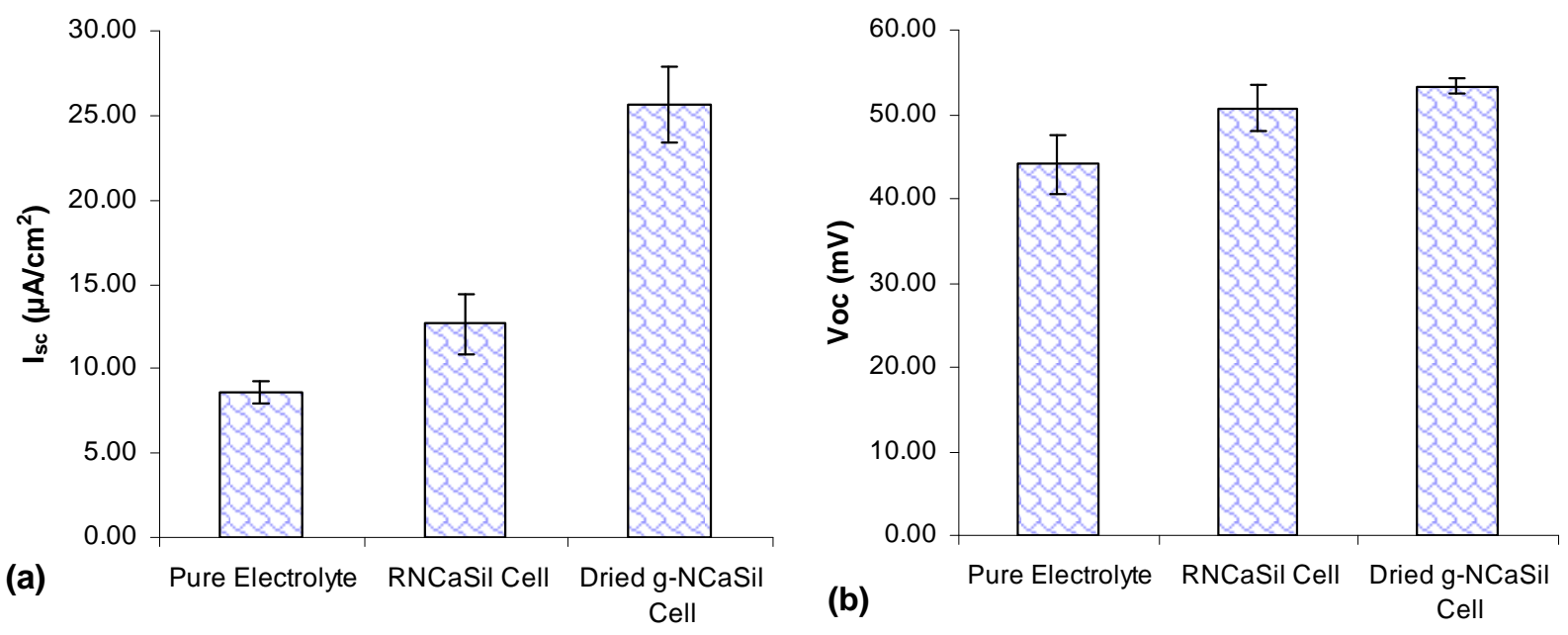

Figure 79. g-NCaSil (dried at $55^{\circ} \mathrm{C}$ ) and RNCaSil with Water-Based Iodide/Triiodide (5 wt $\%$ of $\left.\mathrm{CaCl}_{2}\right)$, and the Electrolyte Alone; (a) $I_{s c}$ and (b) $V_{o c}$.

That the powder/electrolyte cells utilising dried g-NCaSil (Figure 79(a)) achieved greater currents than the more solid-state g-NCaSil tested by itself (Figure 78(a)) was not unexpected. It is well documented that solid-state cells have issues with conductance and with higher proportions of recombination of electron/hole pairs, due to greater difficulties of diffusion of the oxidised triiodide. ${ }^{[96]}$ However, due to advantages in having solid or semi-solid cells (such as reduced sealant issues), the loss of some current could be considered acceptable.

\subsection{Gels Derived from V-and Ce-Doped NCaSils}

As mentioned above (Part IV 8.5) both V- and Ce-treated NCaSil formed gels, after 24 hours of illumination and 288 hours in ambient conditions: g-V-NCaSil and g-Ce-NCaSil. These were tested in gasket cells in their gel states.

g-V-NCaSil exhibited great $I_{s c}$ values, higher than those achieved by g-NCaSil (Figure 80). The potentials obtained were not as high, but were within the range of potentials normally exhibited by NCaSils. Furthermore, the sample proved very stable and gave a consistent performance. Conversely, the g-Ce-NCaSil did not perform so well. Though its $V_{o c}$ was sufficient, its $I_{s c}$ was lower than that of the g-NCaSil and about equivalent to that of the RNCaSil powder/electrolyte cell. It was suspected that the reason for the poor performance was that the gel was too dry. It visibly appeared drier than $\mathrm{g}-\mathrm{V}$ NCaSil and g-NCaSil. It was believed that, had the sample been left on the bench for a lesser amount of time, the current may have been higher. 

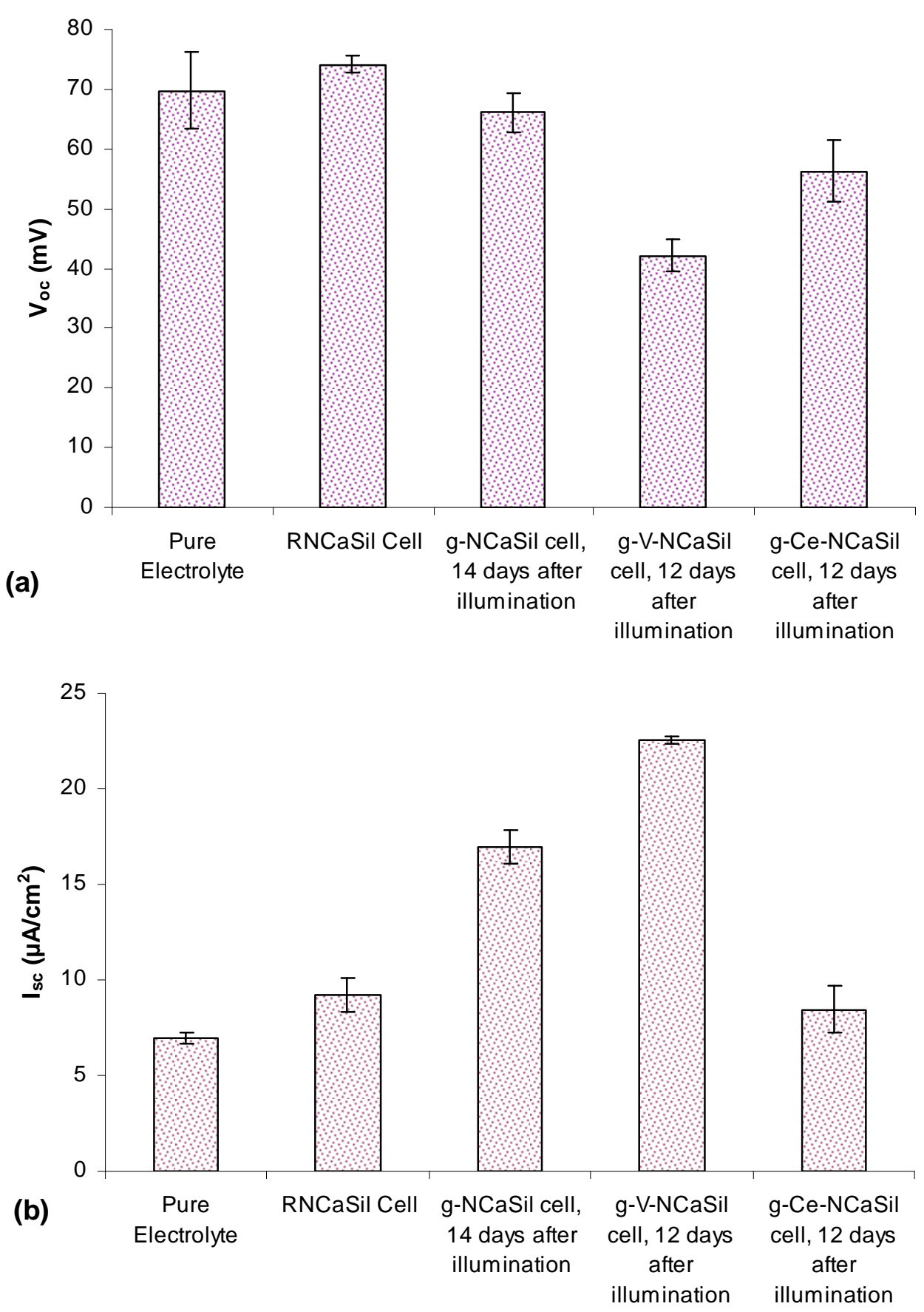

Figure 80. Cells from Gel-State V-NCaSil and Ce-NCaSil; (a) $V_{o c}$ and (b) $I_{s c}$. Note that the g-NCaSil sample was made after 72 hours illumination and tested 14 days after its formation, whereas the g-V-NCaSil and g-Ce-NCaSil were only illuminated for 24 hours and formed gels on the bench over 12 days.

Of course, due to the differences in forming these three gels and the point at which they were each tested, their performances were only illustrative of the potential of such gels. Ideally, one would want to compare gels made under identical conditions, but this was near impossible with the samples left to form gels under ambient conditions, due to the changeability of such conditions. Furthermore, this ideal situation may not be conducive to obtaining the maximum point of operation. The gels may have 
performed more effectively at an earlier or later stage than tested, i.e., when wetter or drier, depending on the form of NCaSil. More work needs to be carried out in this area, under tighter control of operatng conditions, testing the samples periodically over time, in order to find the optimal settings for forming gels from different samples and the point of their maximum cell performances.

\subsection{Effect of the Surrounding Sample}

Because of the way in which beaker cells were constructed, there was sample located between the light source and the photoanode, thought to possibly be inhibiting the amount of light that reached the sample between the electrodes (Part IV 5.2.3). To test whether this was the case, once a g-NCaSil (i.e., semi-solid) sample had been made after 144 hours of illumination, the electrodes and the sample between them was removed and placed into an identical (but empty) beaker, so that the effect of the curvature of the beaker was not removed, but that of the surrounding sample was.

There was little change in potential, as expected, but the current increased by a factor of over two (Figure 81). Thus, it could be concluded that the effect of the surrounding sample was to block the light path. This conclusion was supported by findings referred to earlier (Part IV 5.2.3), where the removal of the sample between the light source and the photoanode in a slotted-vessel resulted in a current over three times as great as that obtained by an equivalent cell that did have sample between the quartz glass window of the vessel and photoanode.

The results also showed that, in terms of measuring efficiency, only the surface area of the electrodes immersed in the sample needed to be considered, and not that of the beaker. In other words, the surface area of the beaker that was illuminated was only indirectly relevant, in that the curvature affected the reflectance of the glass.

When a similar experiment was carried out with another gel (illuminated for 192 hours), but without the use of a new beaker, on removing the surrounding sample, the $V_{o c}$ changed from approximately 68 to $45 \mathrm{mV}$ and the $I_{s c}$ increased from approximately 2.3 to $3.0 \mu \mathrm{A} / \mathrm{cm}^{2}$. However, the intensity of the light on the unprotected sample resulted in very quick drying of the sample. When the light was initially turned on, the $V_{o c}$ was approximately $70 \mathrm{mV}$, but this value declined rapidly. The fast drying of the cell was also likely why the increase in current was not as great as one would have expected, given that the sample was free from any surrounding, light-hindering sample and glass. 

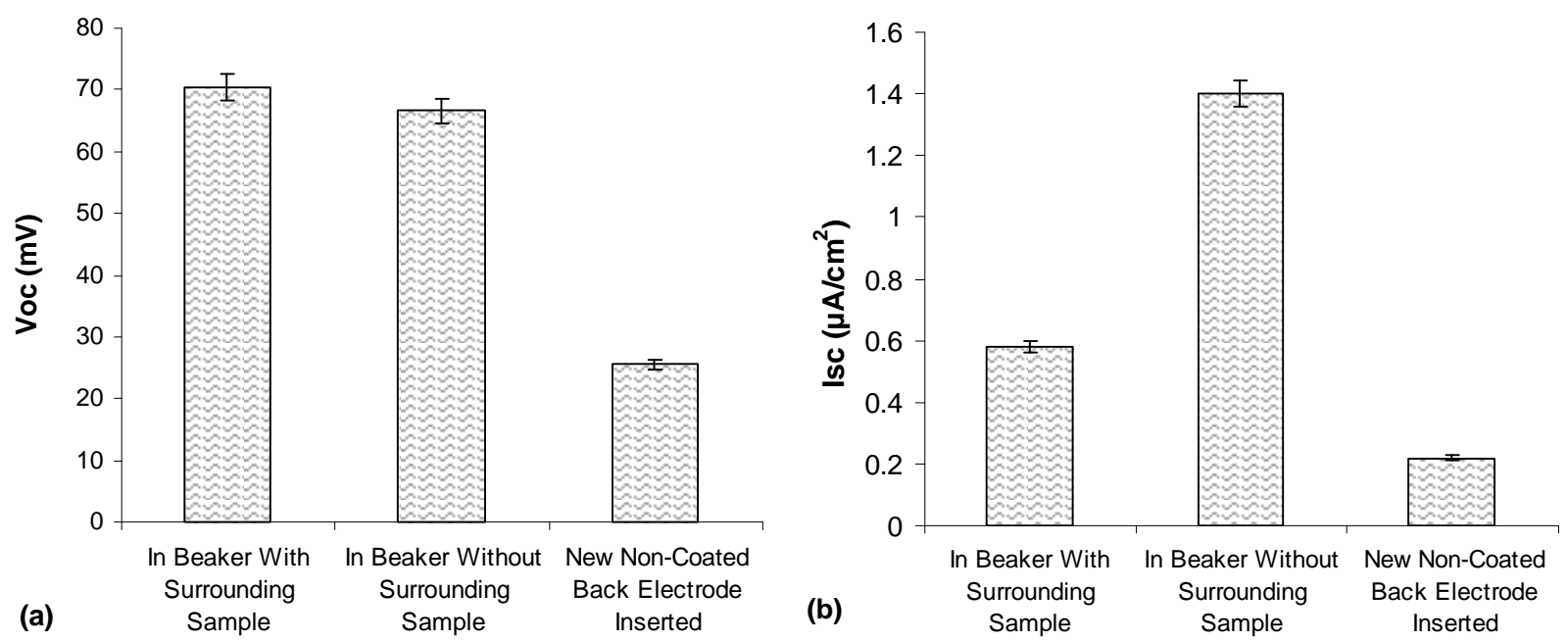

Figure 81. g-NCaSil after 144 hours Illumination; (a) $V_{o c}$ and (b) $I_{s c}$.

With the sample placed in the empty beaker, the dark electrode (C-coated ITO glass) was subsequently replaced by a sheet of un-coated ITO glass. Though cell performance was dramatically reduced, both in terms of the current and potential, the cell did not completely stop working. It was noted that the Ccoating on the original ITO glass had been visibly stripped off. That this had no apparent effect on the cell performance indicated that there must have been enough carbon left on the electrode for the cell to function. The cell continuing to work with an un-coated electrode, inferred that there must have been some carbon floating around in the cell and that this free carbon was somehow enough for some cell function. This made sense, seeing that loss of carbon from the original electrode had been noted. It was also possible that the driving force of the photoactive process of these gel samples was great enough, such that some process was still observed even free of the catalyst on the dark electrode.

\subsection{NMR Analysis of the Gel}

Attempts were made to ascertain the differences in the chemical structures of g-NCaSil, RNCaSil and NCaSil. ${ }^{13} \mathrm{C}$ NMR and ${ }^{1} \mathrm{H}$ NMR analyses showed only the presence of ethanol and water, indicating that ethanol had not been oxidised to acetic acid (see Appendix, Part VII 3, Figures 102 and 103). IR spectra confirmed that no $\mathrm{C}=\mathrm{O}$ moieties were present in any of the samples (see Appendix, Part VII 4, Figure 104). In the NMR spectra, peaks associated with $\mathrm{sp}^{3}$-hybridised $-\mathrm{CH}_{2}-\mathrm{O}\left({ }^{13} \mathrm{C} \mathrm{NMR} \delta 57.3 \mathrm{ppm}\right.$, ${ }^{1} \mathrm{H}$ NMR $\left.\delta 3.5 \mathrm{ppm}\right)$ and $-\mathrm{CH}_{3}$ moieties $\left({ }^{13} \mathrm{C}\right.$ NMR $\delta 16.7 \mathrm{ppm},{ }^{1} \mathrm{H}$ NMR $\left.\delta 1.03 \mathrm{ppm}\right)$ did not exclude the possibility that the ethanol was perhaps interacting with the silicate structures through the alcoholic oxygen. Solid state ${ }^{29} \mathrm{Si}$ NMR studies of dried out samples proved more insightful (Figure 82). 


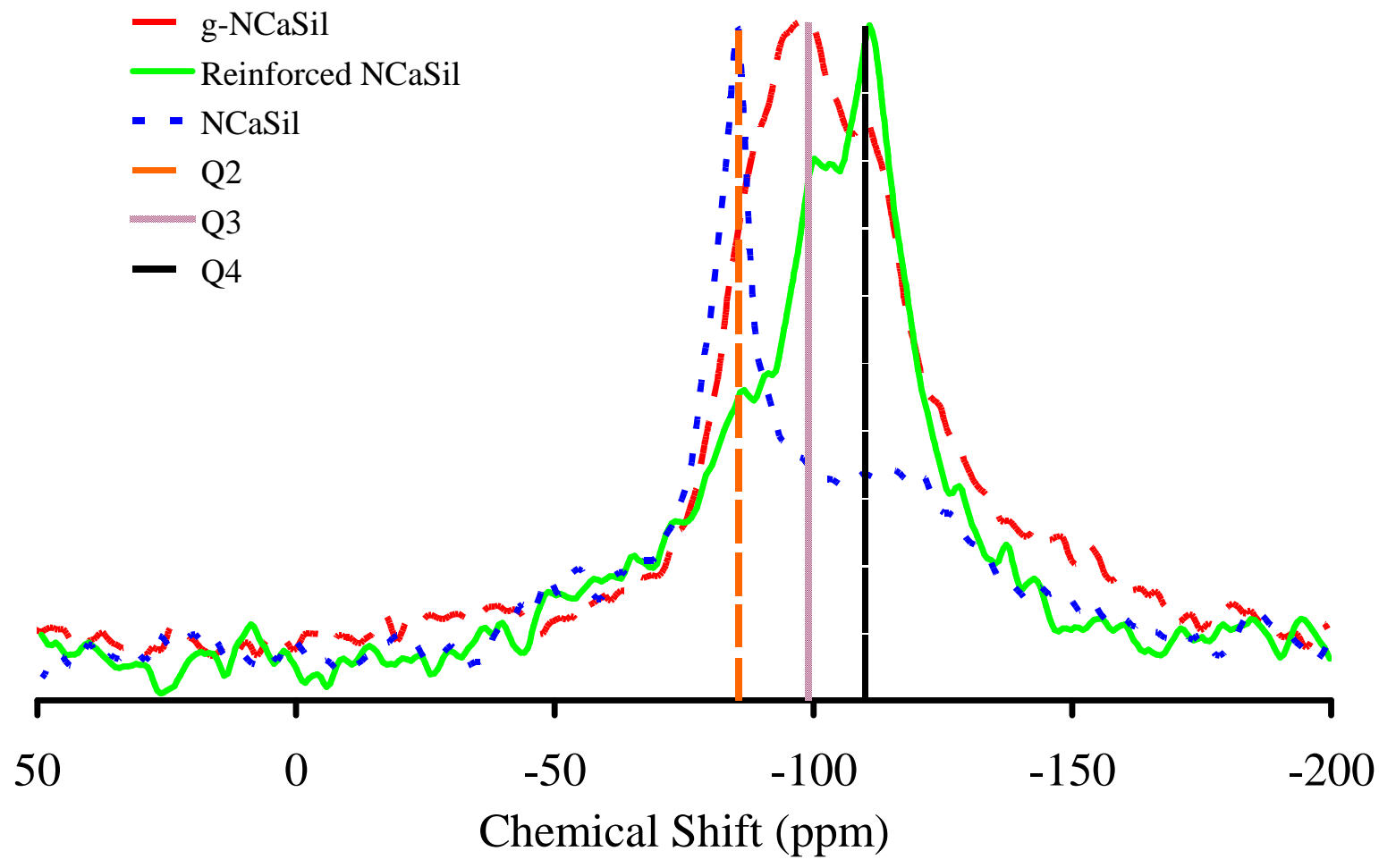

Figure 82. ${ }^{29}$ Si Solid State NMR Spectra of g-NCaSil, RNCaSil and Untreated NCaSil.

The silicon in the three materials compared were in significantly different environments from each other (Figure 82). Untreated NCaSil had almost all its silicon in Q2 environments (meaning it was comprised of chains of corner-bonded silicate tetrahedra, such as found in the mineral wollastonite). ${ }^{[136 \text {, }}$ ${ }^{137]} \mathrm{Q} 4$ environments (typical four corner-bonded $\mathrm{SiO}_{4}$ tetrahedra such as found in cristobalite, $\mathrm{SiO}_{2}$, where all $\mathrm{SiO}_{4}$ tetrahedra are bound to four other $\mathrm{SiO}_{4}$ tetrahedra $)^{[136,137]}$ and $\mathrm{Q} 3$ environments $\left(\mathrm{SiO}_{4}\right.$ tetrahedra are surrounded by three other $\mathrm{SiO}_{4}$ tetrahedrons and one organic or hydroxyl group, as present in silicon oils ${ }^{[136]}$ were only observed at a level of approximately $10 \%$. RNCaSil had predominantly Q4 silicon, with lesser amounts of Q3 followed by Q2 environments (as the reinforcement of $\mathrm{NCaSil}$ deposited a $\mathrm{Q} 4 \mathrm{SiO}_{2}$ layer on the surface of the original material). The gNCaSil formed was largely composed of Q3 centres, the spectrum showed a Q4 environment shoulder off the Q3 peak. This could have been indicative of the formation of a silicon oil related gel, where there was some network in place, but one of low rigidity. Therefore, the ${ }^{29} \mathrm{Si}$ spectrum obtained showed that the silicate structure was different in the gel than in the RNCaSil or NCaSil powders. The close relation to silicon oils raised the question if a corresponding gel could be produced using typical silicon oil forming reactions. This is discussed in the following section. 


\subsection{Synthetic Silicate Gels}

This section discusses attempts to synthetically synthesise the silicate structure of gels derived from RNCaSil and $70 \mathrm{wt} \%$ ethanol (30 wt\% water) iodide/triiodide. NMR studies of the RNCaSil-based gels had suggested a possible correlation of the gels to silicon oils (Part IV 9.4). Therefore, in the first part of this section, the preparation of gels from the condensation of tetraethoxysilane (TEOS) is examined, TEOS being a common precursor to silicon oils. This is followed by a discussion on gels derived from sodium silicate. Both of these gels were also prepared with various metal sources to assess the effect of the presence of different redox couples in the samples, the possible electrochemical interaction of the metals with iodide $\left(e . g ., \mathrm{Cu}^{2+}+2 \mathrm{I}^{-} \rightarrow \mathrm{CuI}_{2}\right.$, the product of which immediately decomposes, $\left.2 \mathrm{CuI}_{2} \rightarrow 2 \mathrm{CuI}+\mathrm{I}_{2}\right)$, ${ }^{[138]}$ and the effect of the sample's colour, which could affect the light absorption range.

\subsubsection{TEOS-Derived Silicate Gels}

\subsubsection{Initial Results}

TEOS was condensed by acid catalysis, the process of which has been well documented in the literature. ${ }^{[118-122]}$ Initial results, using calcium iodide, calcium chloride or calcium hydroxide with TEOS, appeared promising, with the synthetically formed gels displaying currents and voltages even without any electrolyte (iodide/triiodide in $70 \mathrm{wt} \%$ ethanol $30 \mathrm{wt} \%$ water) added. In many cases, the open-circuit voltage $\left(V_{o c}\right)$ decreased as electrolyte was added to the sample (Figure 83(a)), becoming closer to that of the electrolyte alone (approximately $50 \mathrm{mV}$ ), whereas the short-circuit current $\left(I_{s c}\right)$ increased (Figure 83(b)). This indicated that the gels themselves had inherently higher potential differences than the electrolyte, but an inhibited ability to move charge. However, the current improved with the addition of moveable charge carriers. Although the electrolyte was required to allow the current to flow, it was a limiting factor for the cell voltage. Therefore, several cation sources were tested to provide potential electrolyte systems, e.g., $\mathrm{Sr}^{2+}, \mathrm{Mn}^{2+}, \mathrm{Co}^{2+}, \mathrm{Cu}^{2+}, \mathrm{Zn}^{2+}, \mathrm{Fe}^{2+}$ and $\mathrm{Fe}^{3+}$. However, a similar behaviour to the pure calcium cells was observed for several of the other metal sources as well. For example, the voltage of the $\mathrm{Mn}^{2+}$-TEOS gel decreased on the addition of electrolyte solution, but the current increased. 


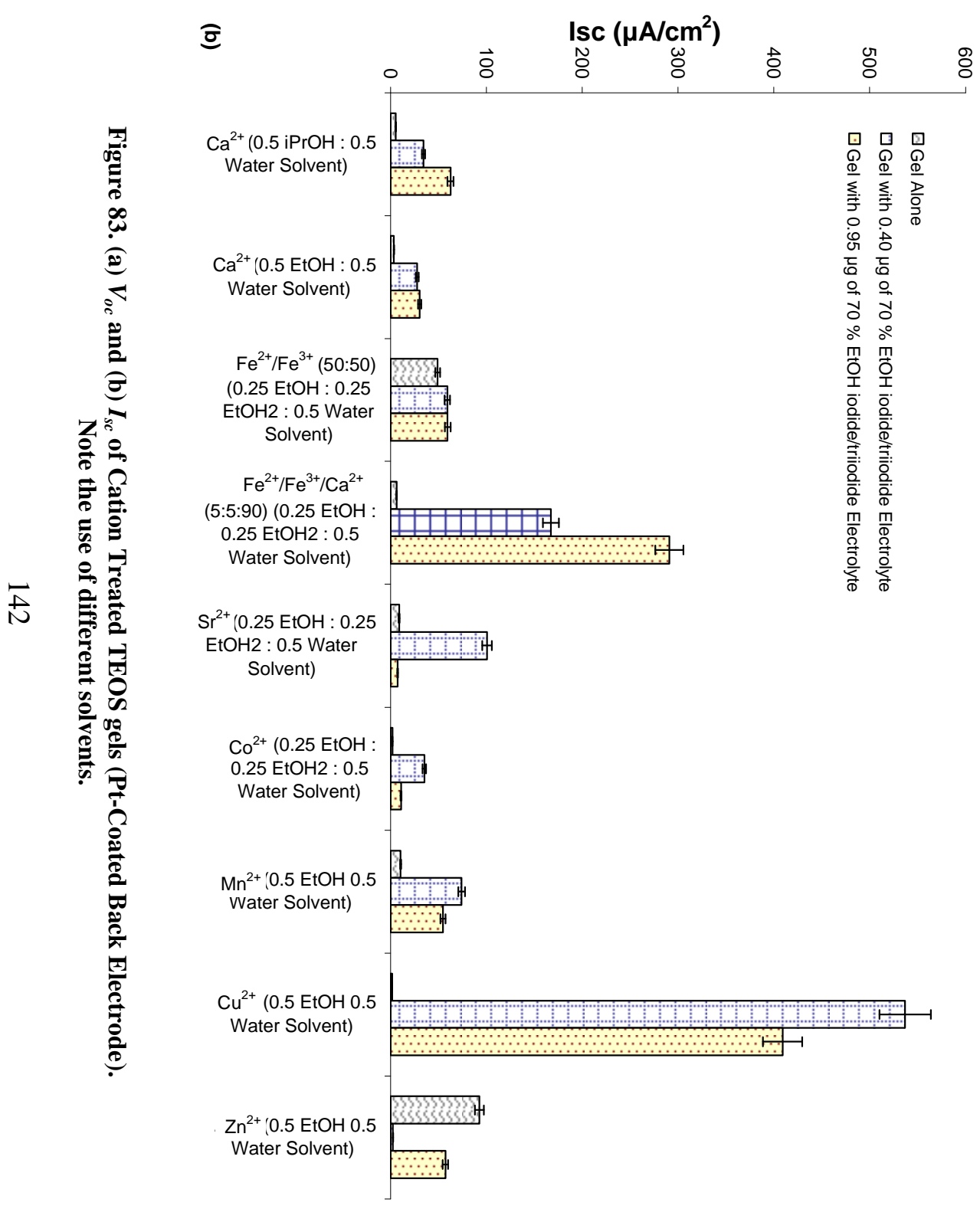

(2)

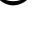

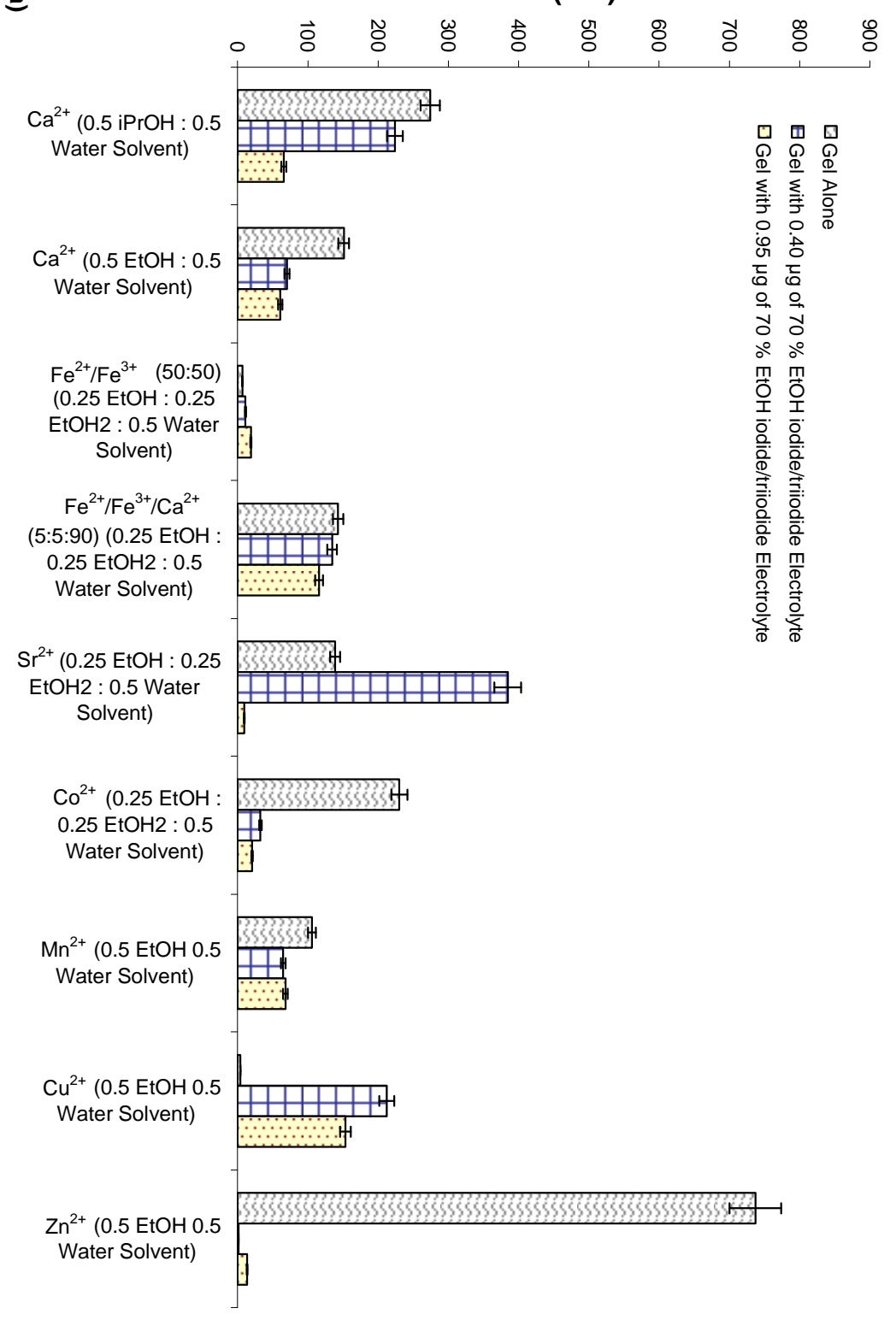


With some samples, the current peaked when only $0.40 \mu \mathrm{g}$ of electrolyte was used and decreased when this amount was increased to $0.95 \mu \mathrm{g}$. This was the case with $\mathrm{Co}^{2+}-\mathrm{Sr}^{2+}-$ and $\mathrm{Cu}^{2}$-TEOS gels. This finding could be explained by the I' concentration rising beyond a maximum point, which caused the viscosity of the sample to increase such that ion mobility was inhibited. ${ }^{[20]}$ The $\mathrm{Zn}^{2+}$-TEOS gel was particularly unusual. It displayed a $V_{o c}$ of almost $740 \mathrm{mV}$ and $I_{s c}$ of over $90 \mu \mathrm{A} / \mathrm{cm}^{2}$ with no electrolyte present. However, the voltage and current both decreased as $0.40 \mu \mathrm{g}$ of electrolyte was added to the gel, but this trend was reversed slightly when more electrolyte was added. This sample is discussed further below.

\subsubsection{Long-Term Testing and the Formation of Batteries}

Despite these initial results, when long-term testing was attempted, products formed after about a week that were not photoactive. Furthermore, it was noticed that the initial currents and voltages of the gels were observable even in the absence of a light source. Thus, it became apparent that the synthetically produced gels were undergoing non-photoactivated electrochemical processes. This was only expected for some of the cells investigated, such as the copper-containing cell, ${ }^{[138]}$ but not for cells involving species such as $\mathrm{Mn}^{2+}$ and the pure $\mathrm{Ca}^{2+}$-based cell. The behaviour of the TEOS cells was in contrast to that of g-NCaSil cells, which were more stable.

The aforementioned $\mathrm{Zn}^{2+}$-treated TEOS gel showed the best cell performance in the absence of any electrolyte, but both the current and voltage became almost non-existent on the addition of $0.40 \mu \mathrm{g}$ of the iodide/triiodide electrolyte, but increased again when $0.95 \mu \mathrm{g}$ of iodide/triiodide electrolyte was added. $\mathrm{Zn}^{2+}$ was able to be reduced by $\mathrm{H}_{2} \mathrm{O}$ (calculated $E_{\text {cell }}=0.024 \mathrm{~V}$ ). On the addition of only $0.40 \mu \mathrm{g}$ of electrolyte, the electrochemical process was suppressed or cancelled out by the photochemical process of the iodide/triiodide couple. When more iodide/triiodide electrolyte was added, it was suspected that the current and voltages observed were predominantly due to this electrolyte, as the values obtained were closer to those observed for the pure electrolyte.

$\mathrm{Fe}^{2+}$ was able to oxidise $\mathrm{H}_{2} \mathrm{O}$ (calculated $E_{\text {cell }}=0.305 \mathrm{~V}$ ) and $\mathrm{Fe}^{3+}$ should have oxidised $\mathrm{H}_{2} \mathrm{O}$ (calculated $E_{\text {cell }}=1.515 \mathrm{~V}$ ) and $\mathrm{I}_{3}{ }^{-}$(calculated $E_{\text {cell }}=0.265 \mathrm{~V}$ ). This explained why the $\mathrm{Fe}^{2+} / \mathrm{Fe}^{3+}$ containing gels showed some current and voltage, which increased with the addition of iodide/triiodide. Given that the $\mathrm{Fe}^{2+} / \mathrm{Fe}^{3+} / \mathrm{Ca}^{2+}$ (5:5:90) gel had so little $\mathrm{Fe}^{2+} / \mathrm{Fe}^{3+}$, it was not surprising that a much lower current was observed for the gel alone than the $\mathrm{Fe}^{2+} / \mathrm{Fe}^{3+}$ (50:50) gel. What was interesting was how much higher the $I_{s c}$ values were for the $\mathrm{Fe}^{2+} / \mathrm{Fe}^{3+} / \mathrm{Ca}^{2+}$ gel than the $\mathrm{Fe}^{2+} / \mathrm{Fe}^{3+}$ gel when electrolyte 
was added, given that the $\mathrm{Ca}^{2+}$ should not have been able to affect any species present in the gel. It was possible that the $\mathrm{Ca}^{2+}$ was merely acting as a charge carrier and its function was enhanced on the addition of the liquid electrolyte.

Three of the synthetically formed gels - namely those based on $\mathrm{Ca}^{2+}, \mathrm{Sr}^{2+}$ and $\mathrm{Mn}^{2+}$ - were not expected to exhibit any electrochemical process at all. Upon closer inspection, IR analysis of the gases released from the TEOS based gels indicated the formation of acetic acid (see Appendix, Part VII 5, Figure 105). Some of the solvent ethanol had been oxidised.

Once the battery processes had faded, all cells remained inactive to light. The addition of the iodide/triiodide electrolyte only showed activity at the level expected for the electrolyte alone.

\subsubsection{Sodium Silicate Derived Gels}

To avoid the side reactions with ethanol, attempts were made to produce gels based on ethylene glycol using sodium silicate. All of these gels also exhibited a release of a carboxylic acid. Different metals were added to the gels, but a blank (or control) was also tested, where no metal had been added. There was no observed current from the gels alone, whether the light was on or not. The gels did exhibit varying potentials, which faded over time. When $0.40 \mu \mathrm{g}$ of electrolyte (70 wt $\%$ ethanol iodide/triiodide) was added, the gels exhibited both current and potential, free from any light source, which deteriorated. The time required for deterioration differed; most samples took around 20 minutes, however some took markedly longer $\left(\mathrm{Mn}^{2+}\right.$ an hour, $\mathrm{Fe}^{2+} 2.5$ hours and $\mathrm{Co}^{2+} 24$ hours). The pre-light processes were allowed to fall to near zero values before the samples were tested under the lamp and the values below measured (Figure 84). Unlike the TEOS gels, these samples were active after the initial electrochemical processes had passed.

The currents and potentials observed for the glycol based cells were found to be significantly lower than those for the g-NCaSil cells, which was expected due to the higher viscosity of ethylene glycol (Table 8). The values were so low, that it was believed that most of the activity observed was due to the small amount of electrolyte present in the sample, rather than any activity of the gels themselves. The cations from the gels assisted in charge transport, but it was not thought that the gels' silicate structures contributed in anyway. 


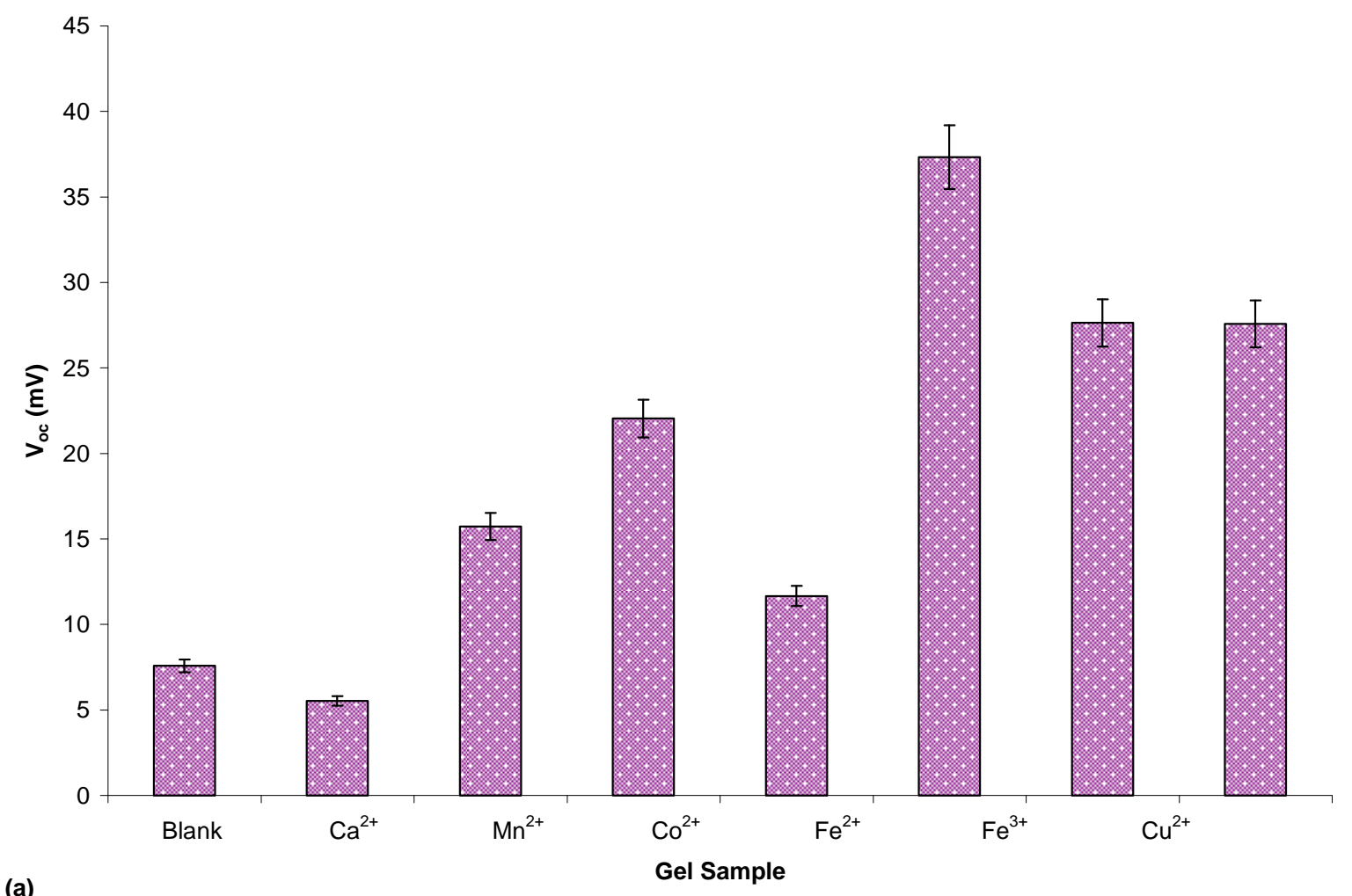

(a)

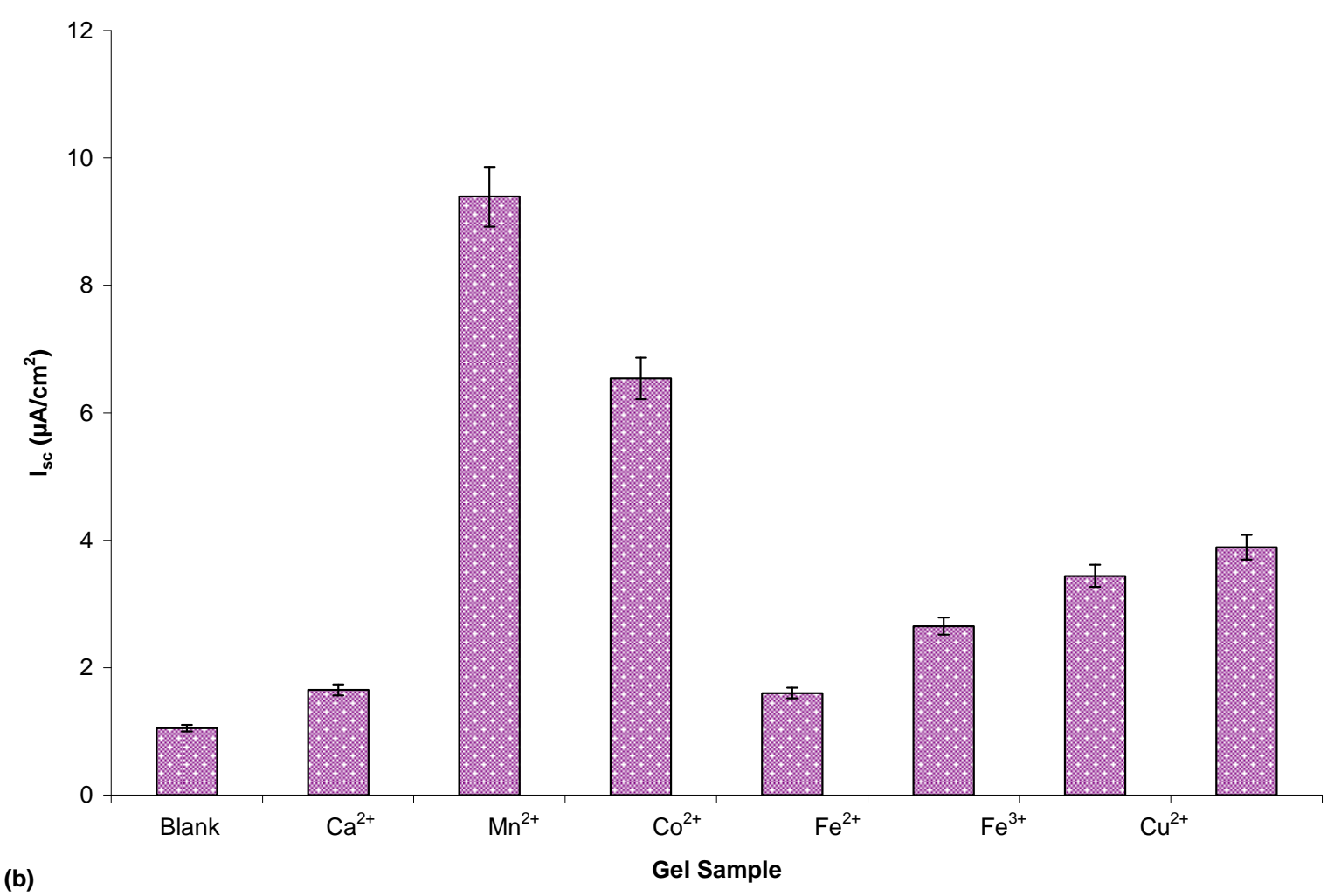

Figure 84. Sodium Silicate Derived Gels Infused with Different Metal Sources (Pt-Coated Back Electrode); (a) $V_{o c}$ and (b) $I_{s c}$. 


\subsubsection{Conclusions}

Overall, the findings regarding synthetically produced gel cells allowed the conclusion to be made that the specific silicate structures of the NCaSils were important to the cell process and that a closer investigation as to the structure of g-NCaSil by X-ray photoelectron spectroscopy (XPS) was required.

\subsection{X-ray Photoelectron Spectroscopy}

XPS carried out on dried g-NCasil samples (from between the electrodes of a beaker cell) confirmed that the NCaSil structure had changed significantly compared to RNCaSil. The Si 2p peaks of the dried gel were more akin to those of $\mathrm{I}_{2}$-treated EENCaSil than RNCaSil (Figure 85). Normal RNCaSil Si 2p had a large silicate peak around $103 \mathrm{eV}(\mathrm{SiOH})$ and a smaller silica peak around $104 \mathrm{eV}\left(\mathrm{SiO}_{2}\right) .{ }^{[15]}$ Silanol was the dominant form observed because of its presence on the surface of the material and the low penetration depth of the spectroscopic method employed. This was in contrast to untreated NCaSil where a large silicate peak at $102 \mathrm{eV}(\mathrm{SiOX})$ was observed, associated with silicon bound over oxygen to electron-withdrawing $\mathrm{Ca}^{2+}$. $\mathrm{I}_{2}$-EENCaSil exhibited a larger peak at $104 \mathrm{eV}$ than $102 \mathrm{eV}$, which was possibly due to $\mathrm{Ca}^{2+}$ binding to the iodine and the loss of SiOX environments. Usually, the contrast between the silica and silicate peaks was quite pronounced. However, with regard to g-NCaSil, the silica peak was not much larger than the silicate peak, indicating that the gel structure resembled both that of RNCaSil and $\mathrm{I}_{2}$-EENCaSil.

The $\mathrm{O}$ 1s spectrum (Figure 86) also indicated that the g-NCaSil was related to $\mathrm{I}_{2}$-EENCaSil. In untreated NCaSil, the main peak was Si-O-Si (around $532 \mathrm{eV}$ ), which was flanked by a smaller Si-O-X peak (around $530 \mathrm{eV}$, where the $\mathrm{X}$ was presumably $\mathrm{Ca}^{2+}$ ) and an even smaller $\mathrm{OH}$ peak (close to 534 $\mathrm{eV}$ ). When treated with iodine, the $\mathrm{Si}-\mathrm{O}-\mathrm{X}$ peak shrank relative to both other peaks, becoming the smallest peak. This was also likely due to an interaction between the $\mathrm{Ca}^{2+}$ and iodine. The spectrum for RNCaSil showed similarly large peaks associated with $\mathrm{Si}-\mathrm{O}-\mathrm{Si}$ and $\mathrm{OH}$ but little $\mathrm{Si}-\mathrm{O}-\mathrm{X}$. The spectrum for g-NCaSil plotted in Figure 86 clearly showed an Si-O-X peak, although it was the smallest of the three present. This supported the earlier assumption that the gel was related to a silicon oil, where Si-Oorganic rest peaks were present. 


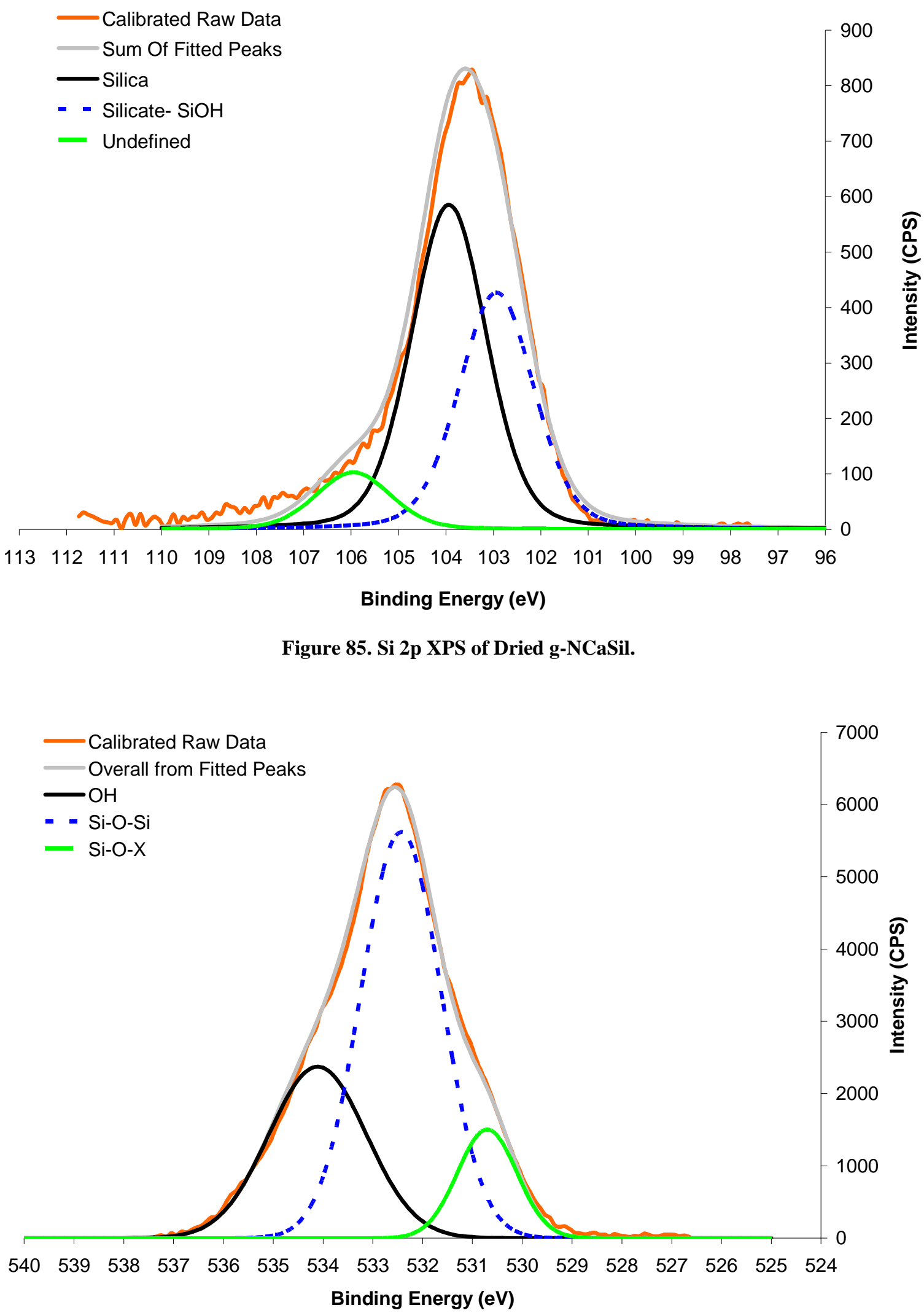

Figure 86. O 1s XPS of Dried g-NCaSil. 
The $\mathrm{Ca}^{2+} 2 \mathrm{p}$ spectrum (Figure 87) showed a $2 \mathrm{p} 1 / 2$ and $3 / 2$ doublet at 351.5 and $348 \mathrm{eV}$, respectively. These peaks in untreated NCaSil and RNCaSil showed up below 351 and $347.5 \mathrm{eV}$ and were shifted up by around $0.5 \mathrm{eV}$ upon treatment of NCaSil with iodine. Interestingly, the peaks for the dried g-NCaSil were shifted to those of the $\mathrm{I}_{2}$-EENCaSil with 351.5 and $347.7 \mathrm{eV}$ respectively. This suggested that, as with $\mathrm{I}_{2}$-EENCaSil, while there was still some coordination of the iodine to the $\mathrm{Ca}^{2+}$ and $\mathrm{Si}$, the iodine was even more closely associated with $\mathrm{Ca}^{2+}$ than in the $\mathrm{I}_{2}$-EENCaSil samples. The shift was not as high as in calcium iodide as a comparison to the X-ray photoelectron spectrum quoted in the literature ${ }^{[139]}$ showed, where the binding energies for calcium were in a range of 355.9 to 358.6 and 352.1 to 356.1 $\mathrm{eV}$ respectively.

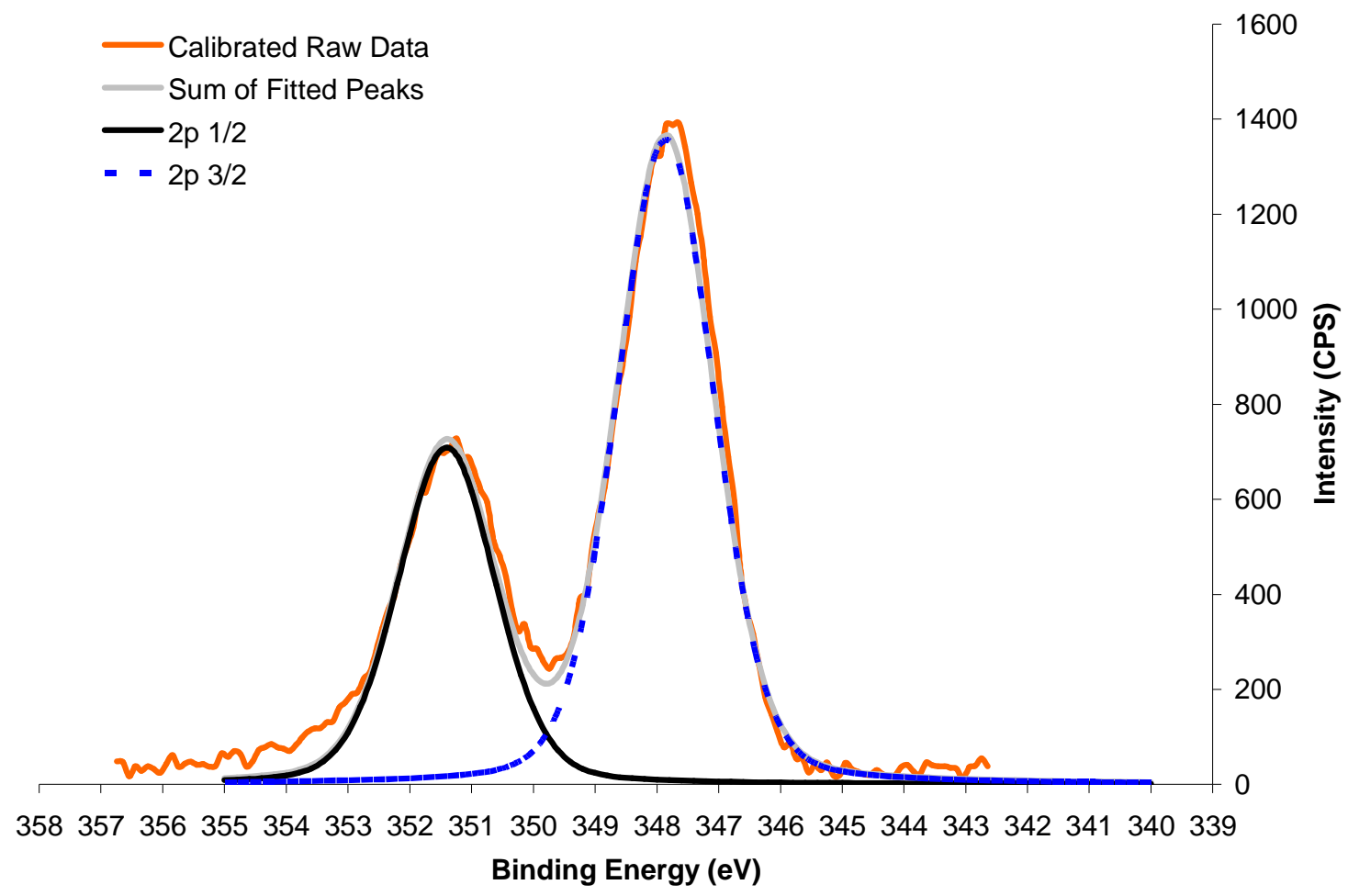

Figure 87. $\mathrm{Ca}^{2+} 2 \mathrm{p}$ XPS of Dried g-NCaSil.

The I $3 \mathrm{~d}$ spectrum of the $\mathrm{g}-\mathrm{NCaSil}$ was similar to that for $\mathrm{I}_{2}$-EENCaSil, both in peak shape and proportion (Figure 88). However, at 619.6 and $631.2 \mathrm{eV}$, the peaks exhibited by g-NCaSil were both higher than those observed from $\mathrm{I}_{2}$-EENCaSil, by around $0.5 \mathrm{eV}$ (as those moieties were 619.2 and $630.6 \mathrm{eV}$, respectively). The peaks additionally differed from those in the literature ${ }^{[139]}$ for I in CaI (624.0 to $624.6 \mathrm{eV}$ and 635.0 to $635.8 \mathrm{eV}$ ). This indicated that the iodine in the g-NCaSil was in environments different from the iodine in $\mathrm{I}_{2}$-EENCaSil and CaI. It was arguable that the calcium and iodine interaction in the g-NCaSil has more CaI character to it than the interaction in the $\mathrm{I}_{2}-\mathrm{EENCaSil}$. 
This was likely because, with regards to $\mathrm{I}_{2}$-EENCaSil, the iodine was introduced in the manufacturing process. Comparatively, the g-NCaSil's formation process likely involved some break down of the NCaSil structure, allowing for a different calcium and iodine interaction.

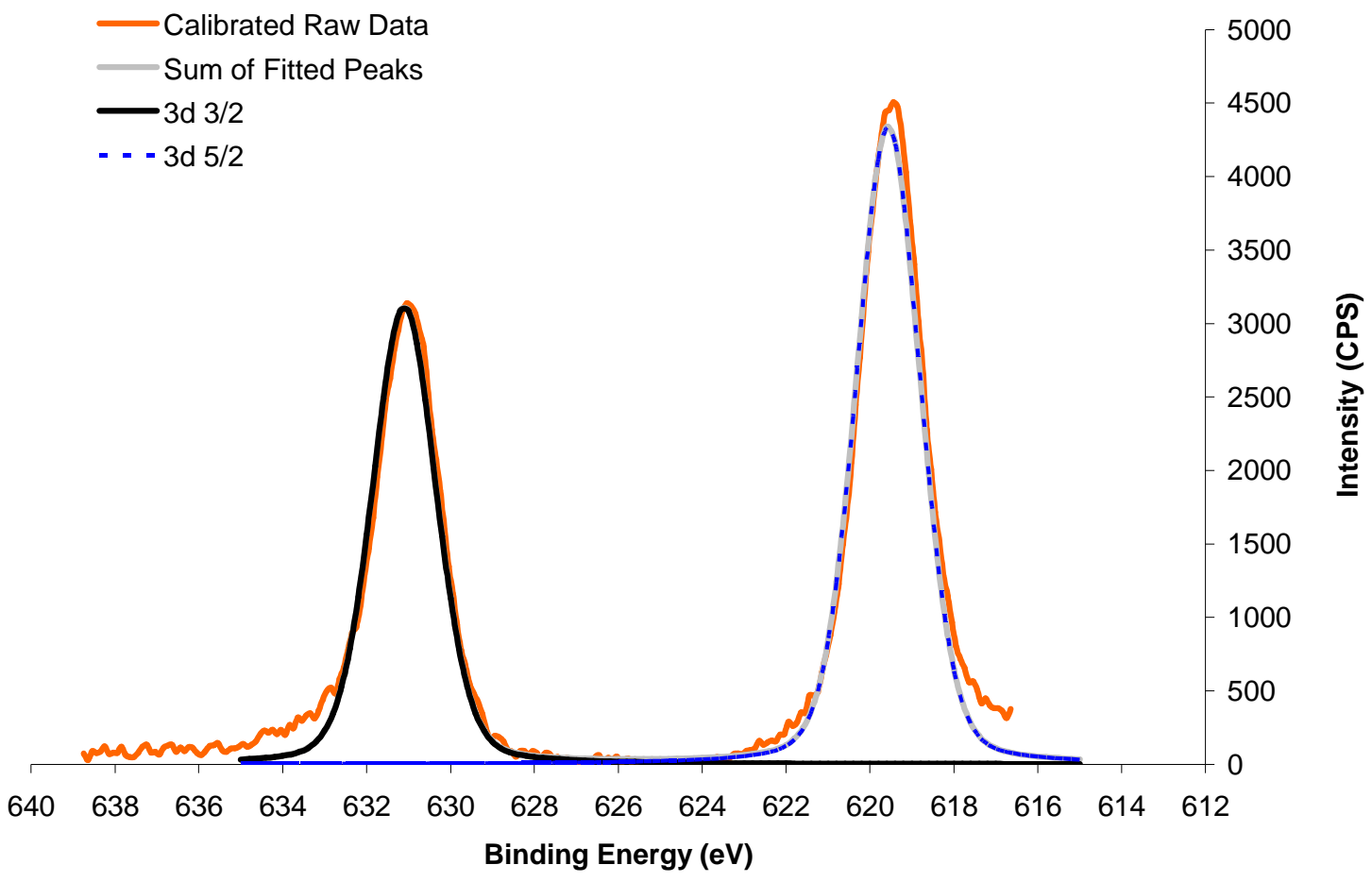

Figure 88. I 3d XPS of Dried g-NCaSil.

In conclusion, XPS showed that, though the gel was more similar to $\mathrm{I}_{2}$-EENCaSil than RNCaSil, it differed from both, its structure possibly lying between the two extremes. That the iodine interaction differed from that in $\mathrm{I}_{2}$-EENCaSil was supported by cyclic voltammetry performed earlier (Part IV 1.1.5). The XPS evidenced that, in g-NCaSil, the iodine added interacted with the $\mathrm{Si}$ and $\mathrm{Ca}$, but predominantly the latter (Figure 89(b)). However, the calcium ions remained bound to the silicate structure. It can be surmised that the retention of binding modes between the calcium ions present and the silicate (as indicated by XPS) in g-NCaSil could be the reason for the activity and stability of gNCaSil, and why the electrochemical decomposition of ethanol was not observed. This was supported by the earlier finding that only the more $\mathrm{Ca}^{2+}$-rich RNCaSil was able to form g-NCaSil (Part IV 1.3.1). Thus, the silicate backbone of NCaSil was important to the cell process and stability and the NCaSil was not merely acting as a source of $\mathrm{Ca}^{2+}$. Furthermore, that the synthetic gels could not emulate the cell performance of the g-NCaSil further illustrated the importance of the iodine-NCaSil interaction and allowed the conclusion to be made that such binding could not occur on just any silicate. 

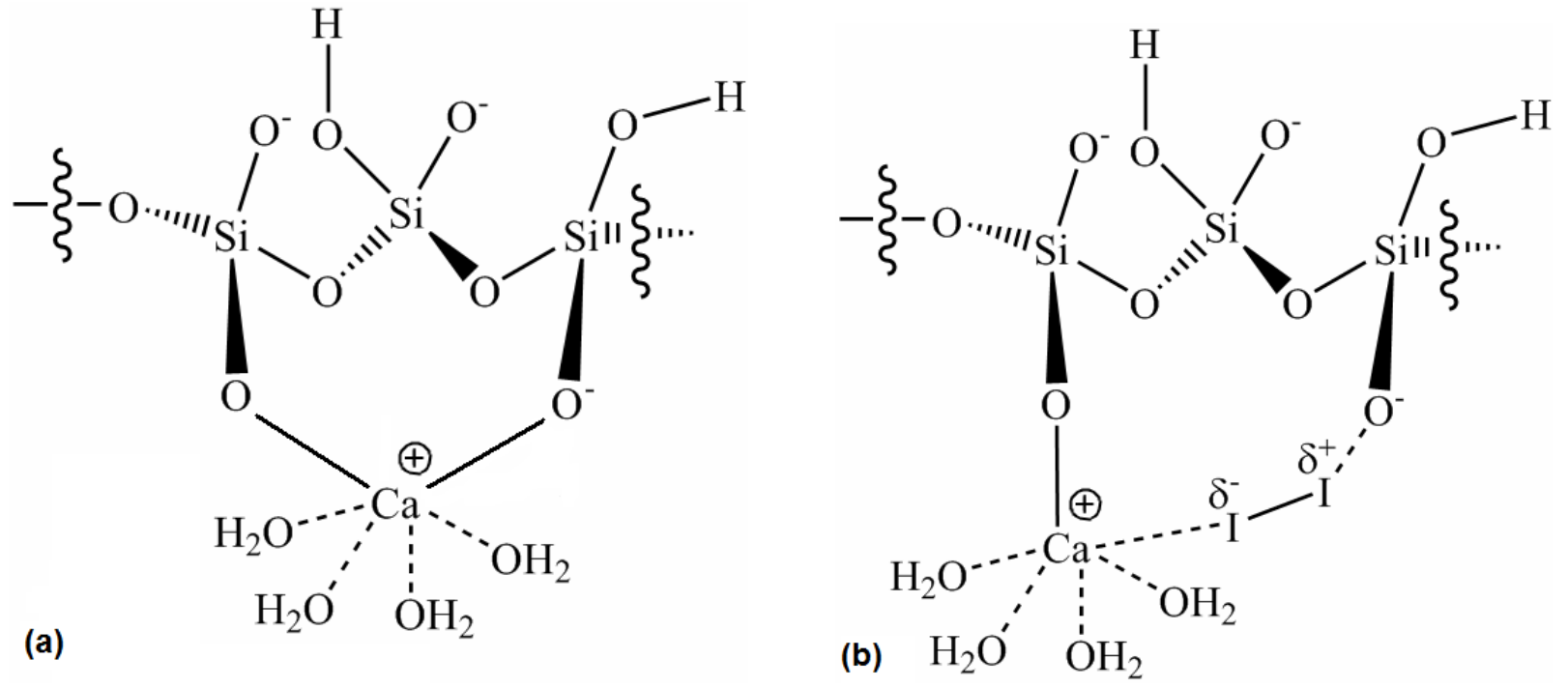

Figure 89. (a) Structure of NCaSil; and (b) Proposed Structure of g-NCaSil.

Interestingly, with DSSCs, triiodide interacting or binding to the semiconductor material would result in recombination of the hole the triiodide carries and the electron in the semiconductor. ${ }^{[68]}$ Water present in DSSCs usually binds to the $\mathrm{TiO}_{2}$, blocking sites from triiodide interaction, decreasing recombination. That the interaction that the silicate had with iodine increased cell performance was, thus, interesting. The likely reason for this was that it was iodine that was interacting with the RNCaSil surface, not triiodide. The generally anionic surface of NCaSils supported this, as the $\mathrm{SiO}^{-}$surface groups would repel triiodide and iodide, leaving them free to carry charge. The same reasoning applied if the cells made were soggy sand electrolytes, as the binding of iodine would leave the iodide and triiodide free to undergo their photogalvanic process. The binding of iodine to the silicate was also thought to be the reason why NCaSils inhibited the electronic transitions of iodine (Part IV 1.1.5), and reduced photobleaching. Furthermore, it inferred that process taking place in the RNCaSil cells was quite different to that of a typical $\mathrm{TiO}_{2}$ DSSC, and more closely related to a soggy sand electrolyte.

\subsection{Energy Dispersive Spectrometry}

Energy Dispersive Spectrometry (EDS) was performed on a dried sample of g-NCaSil and $\mathrm{I}_{2}$ EENCaSil. Relative to silicon, oxygen contents were comparable, however $\mathrm{I}_{2}$-EENCaSil showed the presence of twice as much calcium as the g-NCaSil, whereas the g-NCaSil exhibited twice as much iodine as the $\mathrm{I}_{2}$-EENCaSil. That the g-NCaSil had a greater cell performance indicated that the association of iodine with NCaSil was beneficial to cell performance, and possibly more so than the calcium content of the silicate. 


\subsection{UV-vis}

With the long-term testing of g-NCaSil (168 hours), a colour differential in the beaker developed. The sample was a darker red-brown towards the light source and a paler yellow colour towards the back of the cell (Figure 90). In other words, triiodide migrated towards the light, whereas the dark side of the cell contained only iodide. This was confirmed by UV-vis analysis, which showed that the concentration of iodide $(220 \mathrm{~nm})^{[60]}$ and triiodide $(290 \text { and } 360 \mathrm{~nm})^{[60]}$ differed throughout the beaker cell overtime (Figure 91). When RNCaSil was added to the electrolyte, the proportion of iodide decreased relative to the triiodide. However, this appeared to be reversed after constant illumination for 4 days. After 7 days illumination, the colour differential became apparent. UV-vis spectroscopic analysis of samples from three areas of the beaker was carried out with samples from in front of the photoanode, between the electrodes and behind the dark electrode. It could be seen that, as one moved from the end of the beaker closest to the light to the dark end of the beaker, the triiodide content decreased. There was only a small decrease in triiodide going from the front of the beaker to the area between the electrodes. However, the sample behind the electrodes, towards the dark end of the beaker cell, was almost devoid of any triiodide at all.

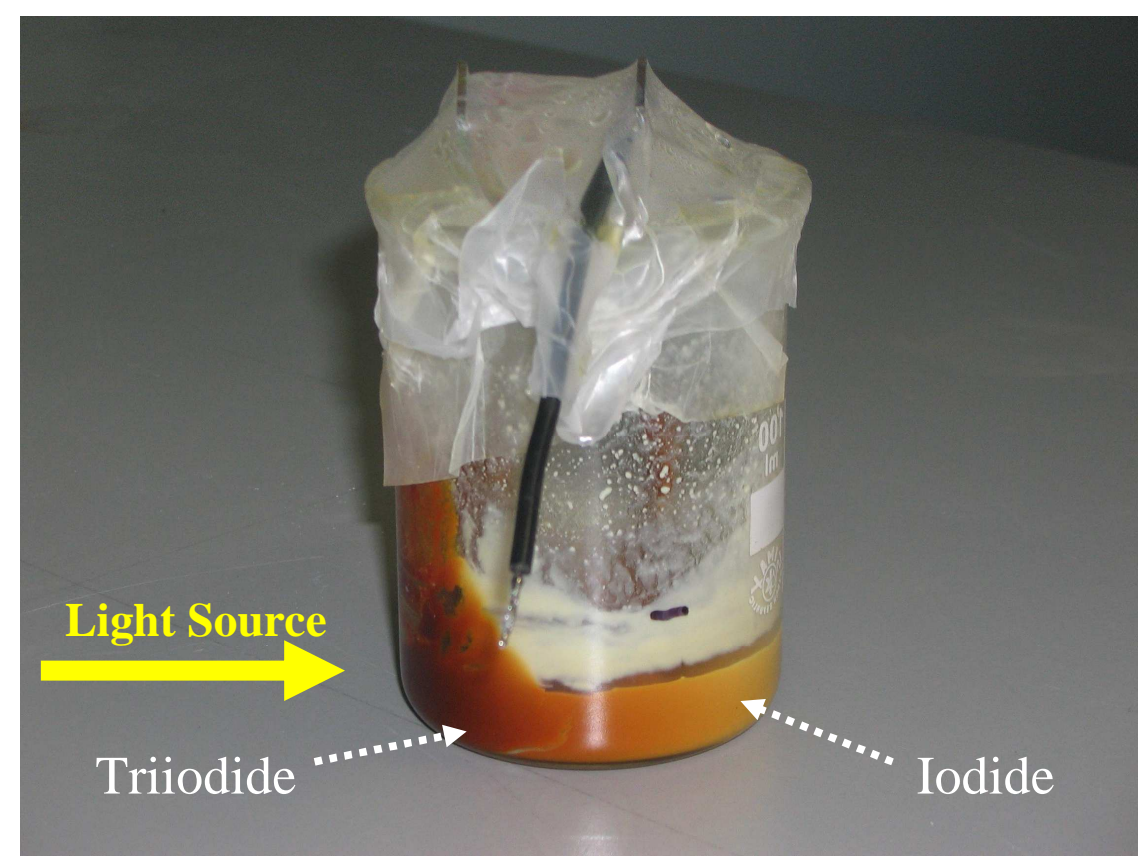

Figure 90. RNCaSil in 70 wt\% Ethanol (30 wt\% Water) Iodide/Triiodide Electrolyte After 168 Hours Illumination.

That triiodide tended toward the light, inferred that it was the photoactive species. ${ }^{[106]}$ If the cell were a DSSC or a semiconductor photogalvanic cell, there would be no reason why the triiodide would 
concentrate near the light source. Triiodide is a photoproduct in DSSCs (when iodide reduces the lightabsorbing material) and partakes in the semiconductor photogalvanic process, however this would only explain the presence of triiodide between the electrodes, not in front of the transparent electrode. Therefore, the spectra indicated that the cell was photogalvanic in nature, and the iodide/triiodide couple dominated. In the photogalvanic process, the triiodide should be regenerated by the combination of iodine and iodide between the electrodes, which explains why it was still present there. However, as there was no regeneration of it behind the dark electrode, overtime it departed this region of the cell. Importantly, this did not appear to affect cell performance. Therefore, the behaviour showed the dependence of the cell on the iodide and triiodide processes, further supporting the soggy sand electrolyte postulation.

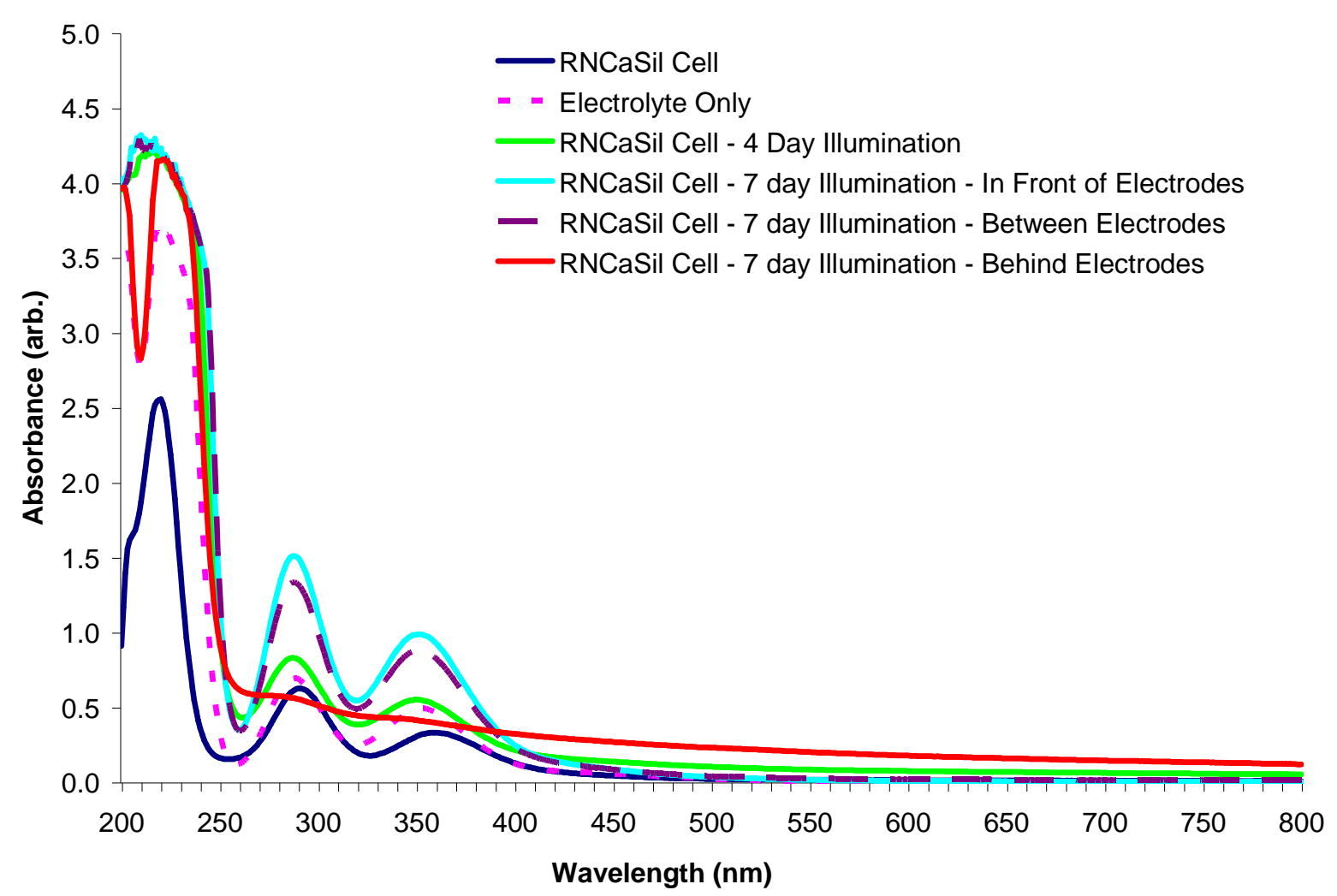

Figure 91. UV-vis of Different Regions of the Beaker of Long-Term Tested g-NCaSil.

\subsection{X-ray Diffraction}

$\mathrm{X}$-ray diffraction was also performed on a dried sample of g-NCaSil, however this did not prove overly insightful. It indicated the presence of ethanol, calcite $\left(\mathrm{CaCO}_{3}\right.$, the presence of which is common in NCaSils) and cristobalite or $\mathrm{Q}^{\mathrm{SiO}_{2}}$ (see Appendix, Part VII 6, Figures 106 and 107). 


\section{Sintering}

If the NCaSils were behaving as semiconducting materials in the cells discussed thus far, a potential drawback of the cells was that they were not sintered to the substrate. Hence, there was a low connectivity between particles and between the semiconducting material and the electrode. Electron/hole pairs generated on a particle with no connectivity to either the electrode or a particle connected to the electrode were not utlised, which resulted in low power conversion efficiencies. Sintering NCaSil to the photoelectrode would have increased cell performance if the NCaSil-containing cells were behaving as DSSCs.

On the other hand, if the NCaSils were not acting as semiconducting materials, but as insulating or network materials in soggy sand electrolytes, the sintering of the NCaSils to the substrate was expected to decrease cell performance, as the NCaSils would no longer be available to interact as greatly with the iodide/triiodide electrolyte.

Here, attempts to increase the connectivity between NCaSil and the electrodes are discussed.

\subsection{Silver Paint}

Using silver paint as a conductive adhesive between NCaSils and the electrode did not work, as the affinity of the silicates surface for silver was too great. Silver blocked the pores of NCaSils, rendering them inactive.

\subsection{Sodium Silicate}

Sodium silicate was tested as a binder of NCaSils to ITO glass. This gave negligible results, both in potential or current. The coating appeared neither very conductive nor photoactive. Sodium silicate is known to dissolve glass, ${ }^{[140]}$ due to its alkalinity. ${ }^{[141]}$ It was suspected that the sodium silicate dissolved ITO off the glass and even dissolved some glass. Furthermore, this methodology gave a rough surface, which made it difficult to achieve good contact with the opposite electrode. 


\subsection{Hydrothermal Conversion}

Hydrothermal treatment of NCaSil was performed at $210^{\circ} \mathrm{C}$ to coat ITO glass (Experimental, Part III 11.3) and desposit a silicate coating of approximately $15 \mu \mathrm{m}$ thickness. This was tested with $70 \mathrm{wt} \%$ ethanol (30 wt\% water) iodide/triiodide in a $0.08 \mathrm{~mm}$ thick gasket. Both the potentials and currents exhibited by the cell were poor and lower than the values one would have expected had the cell consisted of NCaSil powder used in conjunction with the same electrolyte or even of the electrolyte alone (Figure 92).

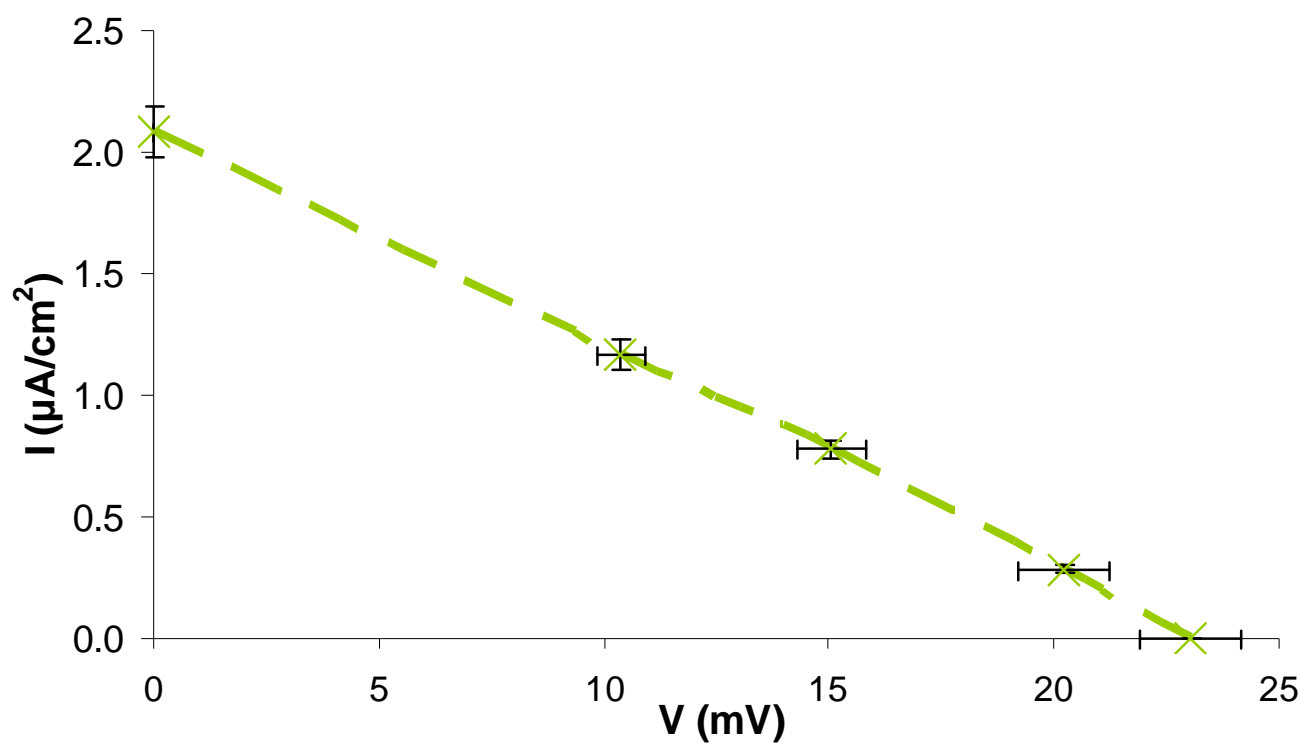

Figure 92. Current versus Voltage of NCaSil Sintered onto ITO Glass with 70 wt\% Ethanol (30 wt\% Water) Iodide/Triiodide (0.08 mm Gasket, Pt-Coated ITO Glass Back Electrode).

The poor performance of the cells suggested that the NCaSil was not acting as a semiconductor. The cell was dominated by the photogalvanic process of the iodide/triiodide, which was inhibited by the layer of NCaSil on the electrode, due to its low conductivity. Furthermore, the sintered NCaSil was not available to act as the network material in a soggy sand electrolyte arrangement. 


\section{Dye Sensitisation}

RNCaSil was dye sensitised with tris(2,2'-bipyridyl)dichloro-ruthenium (II) hexahydrate. As previous experiments had shown that sintering RNCaSil was not beneficial for cell performance, the RNCaSil was not sintered onto ITO glass before immersing in a solution of the dye, as is usually done with DSSCs. ${ }^{[23,40]}$ The RNCaSil alone was left in the dye solution. The resulting yellow-orange powder was tested with $70 \mathrm{wt} \%$ ethanol $30 \mathrm{wt} \%$ water iodide/triiodide (Experimental, Part III 12).

The resultant cells (Figure 93) exhibited lower currents $\left(5.8 \pm 0.2 \mu \mathrm{A} / \mathrm{cm}^{2}\right)$ and voltages $(59.5 \pm 1.0$ $\mathrm{mV})$ than those obtained with un-sensitised RNCaSil cells under the same conditions $(9.2 \pm 0.4$ $\mu \mathrm{A} / \mathrm{cm}^{2}$ and $74.1 \pm 0.8 \mathrm{mV}$, respectively, Part IV 4.4.1).

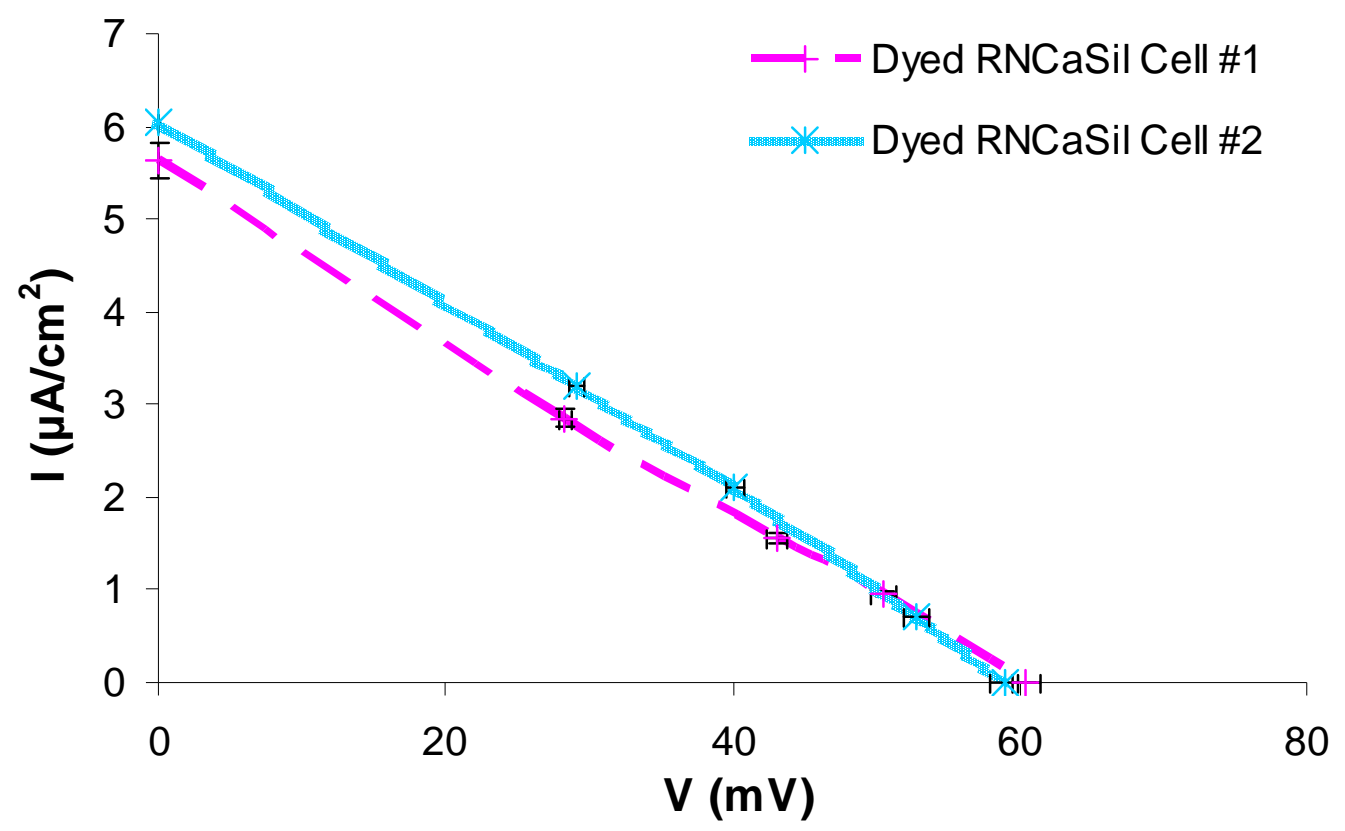

Figure 93. Current versus Voltage of Dye-Sensitised RNCaSil with 70 wt\% Ethanol 30 wt\% Water Iodide/Triiodide (Tested in a Normal Gasket with Pt-Coated Back Electrode).

Given that the evidence, thus far, indicated that the RNCaSil was not acting as a semiconducting material, it was not surprising that dye sensitisation did not increase cell performance. If any electron/hole pairs were generated on the dye through light uptake, the holes may have oxidised the iodide, but the electrons would not have been passed through onto the semiconducting material efficiently or in any way conducive to cell performance (Figure 94). Though the dye complex was positively charged $\left(\mathrm{Ru}^{2+}\right)$, the ligands surrounding the ruthenium were bulky and electron rich, and 
would have been repelled by the anionic surface of RNCaSil. Furthermore, the dye used had no ligands able to bind to the RNCaSil surface. Thus, the dye was likely only loosely bound to the RNCaSil surface, electron transfer from the dye to RNCaSil would have been poor, and recombination rates potentially high. Modification of the dye was likely required.

Additionally, as the RNCaSil was not sintered onto the electrode, the connectivity between the RNCaSil and substrate was poor. Consequently, any electrons that were transferred to the RNCaSil were unlikely to have reached an electrode. Furthermore, the method of dye sensitisation meant that the entirety of the RNCaSil particles were coated with dye, resulting in any generated electrons being somewhat trapped within the RNCaSil particles (Figure 94).

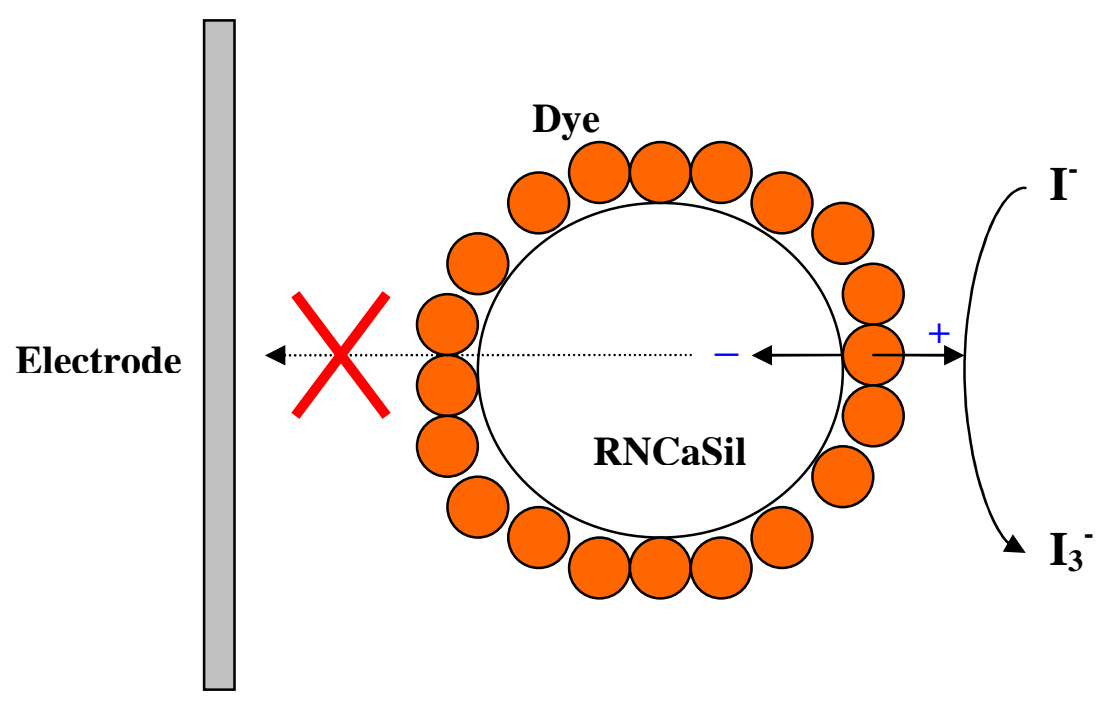

Figure 94. Representation of What May Occur With Dye Sensitised RNCaSil Cells in Iodide/Triiodide.

Therefore, even with dye sensitisation of the RNCaSil, the iodide/triiodide photogalvanic process continued to dominate. Dye sensitisation appeared to lower the efficiency of the iodide/triiodide. This was attributed to light being wasted when adsorbed by the dye rather than triiodide. Furthermore, the decrease in cell performance on dye sensitisation supported the assertion that RNCaSil and the iodide/triiodide formed a soggy sand electrolyte, as sensitising the RNCaSil particles removed its ability to act synergistically with the electrolyte, resulting in data similar to that obtained for the used electrolyte alone $\left(6.9 \pm 0.3 \mu \mathrm{A} / \mathrm{cm}^{2}\right.$ and $69.8 \pm 3.5 \mathrm{mV}$, Part IV 4.4.1). It was also possible that dye sensitisation prevented the RNCaSil particles from acting as a scattering material and increasing the light path in the iodide/triiodide cell. This is discussed further below (Part IV 12.3). 


\section{Conclusions on "Soggy Sand" Electrolytes}

As discussed in the Introduction (Part I 4.3), soggy sand electrolytes using $\mathrm{SiO}_{2}$ or $\mathrm{TiO}_{2}$ show much promise as electrolytes for use in DSSCs. This is due to their stability, increased conductivities and the enhanced cell performances they induce. Although some of the results achieved with RNCaSils and treated NCaSils showed great improvement over the baseline of the electrolytes used alone, none reached the power conversion efficiencies that would be required for commercial viability. It was possible that the reason for this was the limitation of the iodide/triiodide couple. More specifically, that it was potentially the triiodide that was the predominant light absorbing species in these cells and not the NCaSils. In this chapter, evidence discussed so far of the RNCaSil and iodide/triiodide cells functioning as soggy sand electrolytes is summarised. Following this, the RNCaSil cells are compared to $\mathrm{TiO}_{2}$ and $\mathrm{SiO}_{2}$ tested in equivalent cells and the possibility of the NCaSils also acting as a scattering materials briefly expounded.

\subsection{Prior Evidence of Soggy Sand Electrolytes}

Much evidence arose from the experiments already discussed that the use of NCaSils with the iodide/triiodide electrolyte likely formed soggy sand electrolytes, rather than DSSCs or semiconductor photogalvanic cell. This was because two factors were satisfied: (1) the iodide/triiodide redox couple's photogalvanic process dominated the cells's photoactivity and the NCaSils were not acting as semiconducting materials; and (2) the cells were not purely photogalvanic, but exhibited synergy between NCaSil and iodide/triiodide, such that soggy sand electrolytes had been made.

\subsubsection{Dominance of the Photogalvanic Process of the Iodide/Triiodide}

That the iodide/triiodide couple's photogalvanic process dominated cells even in the presence of NCaSils was evidenced by the limited performances shown by the NCaSil-containing cells. All of the potentials obtained from NCaSil-containing cells did not vary greatly from the baseline potentials of the electrolytes alone (Part IV 1.1 and 4.3.1). Though the potentials were sometimes higher, given the wider band gap of NCaSil, a larger difference was expected. The currents of NCaSil-containing cells were also not as high as one would have expected them to be had the NCaSils been acting as semiconducting materials. The curves obtained for plots of $I \times V$ were linear, whether RNCaSil was present in the cell or not (Part IV 1.1.1). This ohmic behaviour is not normally observed in DSSCs, 
which exhibit more rectangular curves. Furthermore, UV-vis performed on the electrolyte with RNCaSil showed no great variance from the UV-vis spectrum of the redox couple alone (Part 1.1.4.2), and the addition of different cations to the electrolyte showed non-DSSC behaviour (Part IV 4.3.2).

When testing the RNCaSil-containing cell with wavelength cut-off filters, the decrease in power output occurred at similar wavelengths as reported in the literature for the iodide/triiodide photogalvanic cell (Part IV 1.1.3). Additionally, the colour differential and iodide/triiodide gradient that arose in the beaker cell from the long-term testing with g-NCaSil strongly supported the dependence on the iodide/triiodide couple, as it could not have been explained if the NCaSil were acting as a semiconducing material either in a DSSC or photogalvanic cell.

Finally, the decrease in cell performance on sintering or using a dye sensitiser further indicated that the NCaSils were not acting as semiconducting materials.

\subsubsection{A Soggy Sand Electrolyte}

The NCaSil-containing cells did not merely exhibit the cell performance of the iodide/triiodide redox couple, but showed evidence of being synergistic in nature and soggy sand electrolytes. Particularly indicative was the fact that current decreased upon adding only a small amount of NCaSil to the electrolyte, but increased once a certain NCaSil to electrolyte ratio had been reached (Part IV 4.1). When only a small quantity of NCaSil was used, NCaSil impeded the photogalvanic process of the iodide/triiodide couple, but at a certain ratio a synergistic effect was attained and a cell performance greater than that of the electrolyte alone was observed.

Performances of the NCaSil cells were typically enhanced over the electrolyte solutions alone because of favourable interactions of the electrolyte with the NCaSils, such that conductivity was increased. Potentials could have been enhanced slightly due to some photoactivity of the NCaSils, which had wider band gaps than the triiodide/iodide. However, the differences in potentials fell into experimental error and, overall, the cell behaviour was dominated by the iodide/triiodide couple. The NCaSils had alkaline surfaces, such that they were unlikely to attract iodide or triiodide to their surfaces, but rather potassium (KI was used as the iodide source). This further explained why XPS showed that it was iodine that interacted with NCaSils, not iodide/triiodide and why the iodine and NCaSil interaction was so important in g-NCaSil (Part IV 9.6). The separation of the potassium from iodide and triiodide 
allowed the latter two species to move more freely throughout the cell, increasing conductivity. Thus, the combination of iodide/triiodide and NCaSil acted like a soggy sand electrolyte.

Furthermore, the cells displayed behaviour different from what one would have expected of a purephotogalvanic process. For example, on testing with the wavelength cut-off filters, the decline in power output exhibited was convex to the origin, when the literature had reported a concave curve for the pure electrolyte (Part IV 1.1.3). Additionally, the NCaSil cells showed non-DSSC and non-photogalvanic behaviour on changing the cell surface area and electrode distance (Part IV 5).

\subsection{Comparison to $\mathrm{TiO}_{2}$ and $\mathrm{SiO}_{2}$}

As a rough test for the feasibility of the assertion that NCaSil and iodide/triiodide cells were behaving as soggy sand electrolytes, equivalent cells to the NCaSil cells in 0 or 5 wt $\% \mathrm{CaCl}_{2}$, water-based iodide/triiodide were fabricated and tested with $\mathrm{SiO}_{2}$ (precipitated) and $\mathrm{TiO}_{2}$ (anatase). Though potentials were comparable (Figure 95(a)), the powder NCaSil cells did not exhibit greater currents than the equivalent $\mathrm{SiO}_{2}$ and $\mathrm{TiO}_{2}$ cells (Figure 95(b)). It was only the metal-treated $\mathrm{NCaSils}$ that exhibited greater cell performances. The potentials obtained were quite comparable, indicating that it was predominantly the iodide/triiodide electrolyte that determined the potential. Furthermore, both the $\mathrm{SiO}_{2}$ and $\mathrm{TiO}_{2}$ cells showed linear current over potential plots, comparable to the iodide/triiodide electrolyte alone or when with an NCaSil, indicating that it was also the iodide/triiodide process than governed all of these cells.

Battacharyya et el. ${ }^{[88]}$ found that electrolyte containing $\mathrm{SiO}_{2}$ achieved a greater conductivity than $\mathrm{TiO}_{2}$, due its more acidic surface $(\sim \mathrm{pH} 3)$ having a greater interaction with anions. This meant that its cations were free to move charge. However, our results showed a greater current from the $\mathrm{TiO}_{2}$-containing electrolyte, when free from the $\mathrm{CaCl}_{2}$ additive. Differences could be attributed to the $\mathrm{LiClO}_{4}$ electrolyte used by Battacharyya et al. and probable differences between the $\mathrm{TiO}_{2}$ and $\mathrm{SiO}_{2}$ samples tested in this study and those used by Battacharyya et al. In the work presented here, surface area and particle size analysis of the $\mathrm{SiO}_{2}$ and $\mathrm{TiO}_{2}$ used showed that, though the latter had a lower surface area (BET isotherm of 11 compared to $81 \mathrm{~m}^{2} / \mathrm{g}$ ), it consisted of much smaller particles ( $c a .0 .1 \mu \mathrm{m}$ compared to 3$4 \mu \mathrm{m}$, Figure 96). That the $\mathrm{TiO}_{2}$-containing electrolyte exhibited a greater current implied that the dominating feature of the cell performance was particle size rather than surface area. This postulation was supported by the earlier findings of variances in cell performance in CM-RNCaSil, when samples had similar surface areas but varying particle sizes (Part IV 1.3.2). 

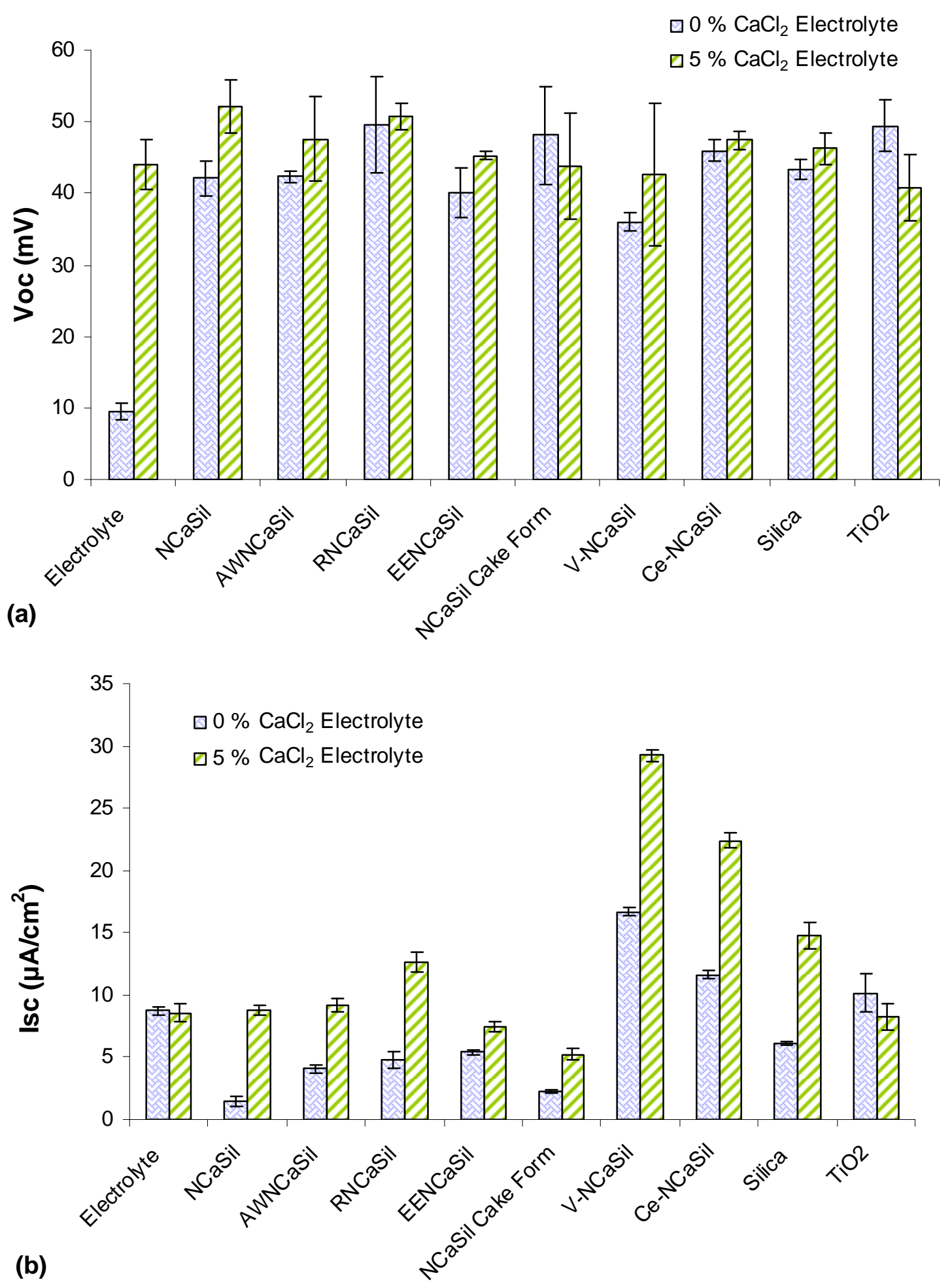

Figure 95. Comparison of $\mathrm{SiO}_{2}$ and $\mathrm{TiO}_{2}$ in $5 \mathrm{wt} \%$ Iodide/Triiodide Electrolyte; (a) $V_{o c}$ and (b) $I_{s c}$.

On the addition of $5 \mathrm{wt} \% \mathrm{CaCl}_{2}$, large increases in current were observed for the $\mathrm{SiO}_{2}$, but decreases obtained from the $\mathrm{TiO}_{2}$-containing electrolyte. This was attributed to the more favourable surface interactions of $\mathrm{SiO}_{2}$ with $\mathrm{Cl}^{-}$rather than $\mathrm{TiO}_{2} . \mathrm{TiO}_{2}$ is only mildly acidic ( pH 5). Hence its 
interactions with anions were only very weak, and adding $5 \mathrm{wt} \% \mathrm{CaCl}_{2}$ arguably only increased the viscosity of the sample without increasing the number of available charge carriers.

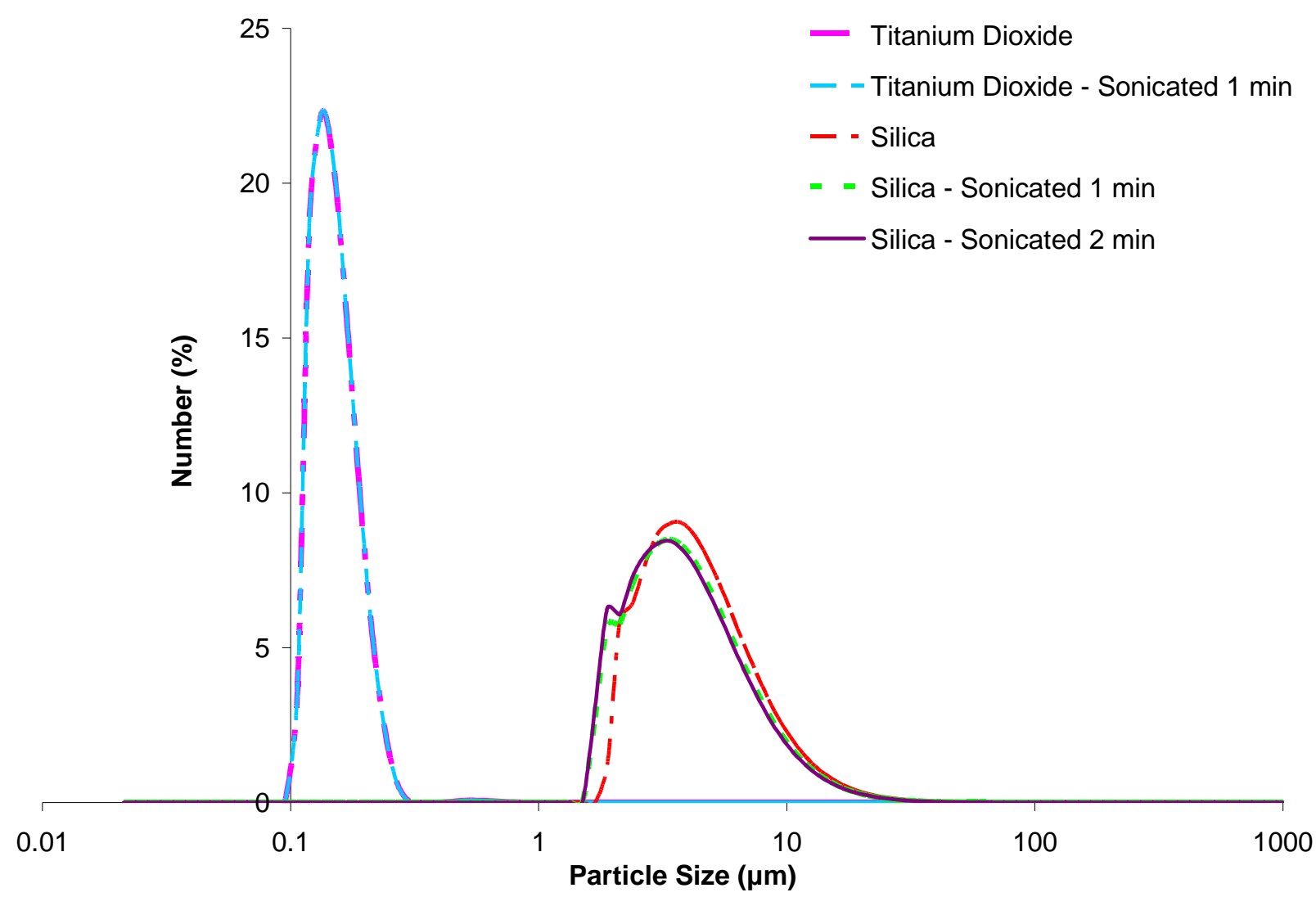

Span:

1.668

Surface Weighted Mean D[3,2]:

$12.335 \mu \mathrm{m}$

d(0.1):

$2.026 \mu \mathrm{m}$
Uniformity:

0.549

Vol. Weighted Mean D[4,3]:

$21.763 \mu \mathrm{m}$

d(0.5):

d(0.9):

$3.683 \mu \mathrm{m}$
$8.171 \mu \mathrm{m}$

Figure 96. Particle Size Distribution Analysis of $\mathrm{TiO}_{2}$ and $\mathrm{SiO}_{2}$.

Interfacial conductivity enhancement rationalised why the NCaSils always showed an improvement in current on the addition of $5 \mathrm{wt} \% \mathrm{CaCl}_{2}$ to the water-based electrolyte and also the observations that, free of $\mathrm{CaCl}_{2}$, the best cell performance was achieved by the NCaSil with the highest surface area, but, with $5 \mathrm{wt} \% \mathrm{CaCl}_{2}$, the determining feature of cell performance was the inherent stability of the NCaSil (Part IV 1.1). The importance of the retention of $\mathrm{Ca}^{2+}$ to the NCaSil structure for long-term stability and activity, due to the interaction between iodine and surface-bound $\mathrm{Ca}^{2+}$, was supported by XPS of 
the g-NCaSil (Part IV 9.6). Furthermore, NCaSils have a very alkaline surface, thereby attracting cations. When no $\mathrm{CaCl}_{2}$ was added to the electrolyte, the driving force for the removal of $\mathrm{Ca}^{2+}$ from the surface of NCaSils was greater. Thus, cell current was determined by the $\mathrm{Ca}^{2+}$ accessibility (and surface area) of the NCaSil, due to the increase in conductance caused by the presence of $\mathrm{Ca}^{2+}$ in the electrolyte. This further explained why the currents obtained were so low, as there was likely not as great a synergistic effect between the $\mathrm{NCaSil}$ and iodide/triiodide electrolyte, free from $\mathrm{CaCl}_{2}$. However, when $\mathrm{CaCl}_{2}$ was pre-added to the water-based electrolyte, the NCaSils that were stable when in liquids were able to retain more $\mathrm{Ca}^{2+}$ on their surfaces, thus allowing for the formation of soggy sand electrolytes and higher currents.

That the V-and Ce-treated NCaSils did exhibit increases in current on their addition to the electrolyte, when free of $\mathrm{CaCl}_{2}$, was attributed to the ability to lose $\mathrm{V}$ and $\mathrm{Ce}$ from their structures, thus retaining some of the silicate-bound $\mathrm{Ca}^{2+}$.

g-NCaSils were formed in $70 \mathrm{wt} \%$ ethanol (30 wt $\%$ water) solvated iodide/triiodide, free from any $\mathrm{CaCl}_{2}$. The ability to retain $\mathrm{Ca}^{2+}$ in the electrolyte solution, even though free of a $\mathrm{Ca}^{2+}$ additive was attributed to the lower dissolution of $\mathrm{Ca}^{2+}$ in ethanol than water and also the evaporation of some solvent, which was required for gel formation. The interfacial conductivity enhancement between $\mathrm{NCaSils}$ and the iodide/triiodide also assisted in clarifying why both the water-based and $70 \mathrm{wt} \%$ ethanol iodide/triiodide showed peak performances when $5 \mathrm{wt} \%$ of $\mathrm{CaCl}_{2}$ was added to the water content of the electrolytes. As well as $\mathrm{Ca}(\mathrm{OH})_{2}$ precipitating out at concentrations much greater than 5 wt $\%$ in $\mathrm{Ca}^{2+}$, the $5 \mathrm{wt} \%$ was likely around the equilibrium amount of $\mathrm{Ca}^{2+}$ required to allow for RNCaSil to retain $\mathrm{Ca}^{2+}$ on its surface to form soggy and electrolytes.

$\mathrm{SiO}_{2}$ has tended to be the oxide of choice for soggy sand, semi-solid electrolytes. ${ }^{\left[77,{ }^{88-90]}\right.} \mathrm{RNCaSil}$ competed quite nicely with it in an equivalent set up. However, metal treated V- and Ce-NCaSil currents far surpassed that of the $\mathrm{SiO}_{2}$-containing cell. This was particularly the case with $\mathrm{V}-\mathrm{NCaSil}$, which exhibited currents twice as great as those of the $\mathrm{SiO}_{2}$-containing cells, with or without $5 \mathrm{wt} \%$ $\mathrm{CaCl}_{2}$. For stability reasons, both the $\mathrm{g}-\mathrm{V}$ - and $\mathrm{g}$-Ce-NCaSils are likely of more interest for such application. Also of interest is the use of the g-NCaSil derived from RNCaSil, which gave a cell current of over $25 \mu \mathrm{A} / \mathrm{cm}^{2}$. Furthermore, g-NCaSil was made from an ethanol solvent (70 wt\%), which has been shown to form stable soggy sand electrolytes with iodide/triiodide and $\mathrm{SiO}_{2} \cdot{ }^{[00]}$ Therefore, using NCaSil with iodide/triiodide or a gel form NCaSil as the electrolyte of a DSSC could be investigated in the future. 


\subsection{NCaSils Acting as Scattering Materials?}

It was also possible that the NCaSils were acting as scattering materials in the soggy sand electrolytes. Particles that scatter and lengthen the path of light can increase the spectral absorbance range of cells. ${ }^{[142]}$ For example, in DSSCs, Kang et al. ${ }^{[142]}$ used relatively large particles of $\mathrm{TiO}_{2}$, approximately 70 to $100 \mathrm{~nm}$ in size, as light-scattering particles with smaller, high surface area $\mathrm{TiO}_{2}$ particles $(30 \mathrm{~nm})$. This was to increase absorption between 600 to $800 \mathrm{~nm}$. They managed to achieve a $28 \%$ increase in cell performance as compared to a nanocyrstalline film cell.

Changes in power output on using wavelength filters (Part IV 1.1.3) and UV-vis of RNCaSil cells (Part IV 1.1.4.2) indicated that the NCaSils did not widen the spectral absorbance range of the electrolyte, as the results obtained (with respect to wavelengths) were consistent with the iodide/triiodide alone. However, it was possible that the NCaSils increased the normal light absorbance of the redox couple. The UV-vis reflectance spectrum of RNCaSil (Figure 97) showed that it reflected over $90 \%$ of light of wavelengths between 200 to $800 \mathrm{~nm}$. The reflectance spectrum obtained of RNCaSil was not what was expected given the absorbance spectrum (Part I 4.1). The spectra exhibited high reflectance and absorbance across the entire spectrum, with values adding up to over $100 \%$. Why the spectra were not mirror images of each other was not known. The lowest region of reflection, at approximately $255 \mathrm{~nm}$, was comparative to the highest absorbance peak observed, at approximately $272 \mathrm{~nm}$. However, at $310 \mathrm{~nm}$, the reflectance and absorbance spectra both exhibited peaks.

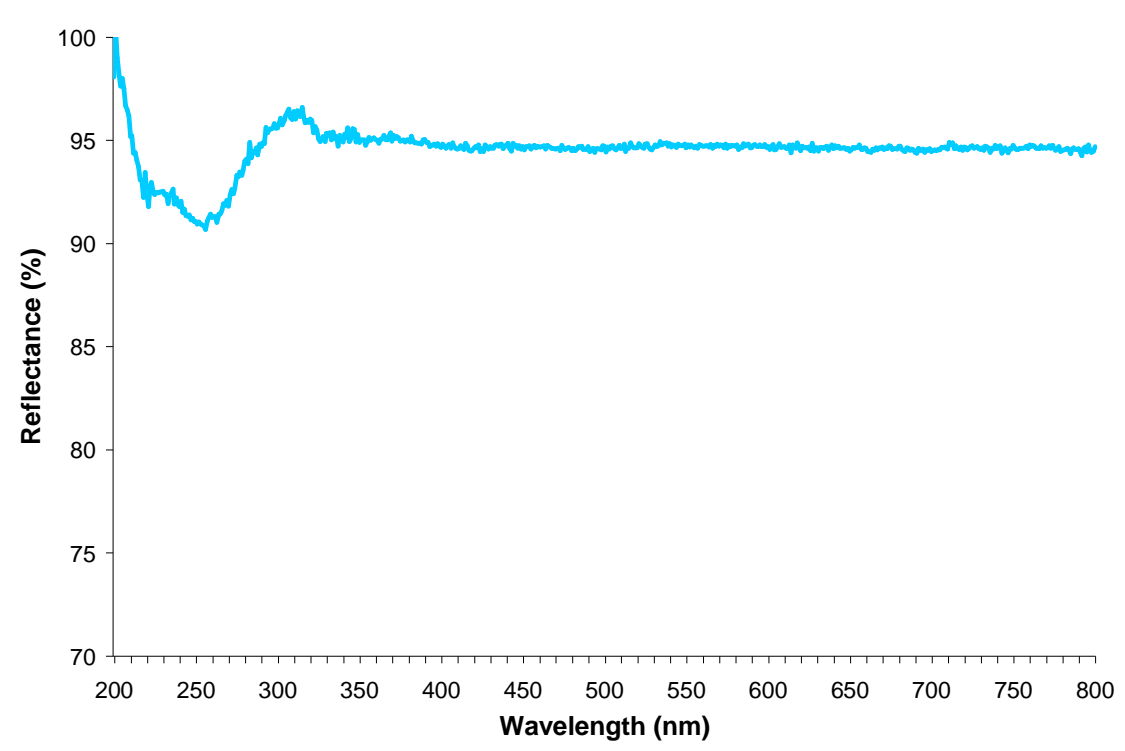

Figure 97. UV-vis Reflectance of Dry RNCaSil (Note the Y-Axis Scale Starts at 70 \%). 
It was concluded that the NCaSils were predominantly acting as scattering agents. This was for three reasons. Firstly, the reflectances observed were higher than the absorbances across the entire spectrum. Secondly, experimental work with NCaSil cells had shown that the NCaSils were not acting as semiconducting materials, inferring that their photoactivity was not contributing to cell performance. Thirdly, prior evidence had shown that particle size was more determinative of cell performance than surface area (Part IV 1.3.2 and Part IV 12.2), suggesting that scattering occurred and not absorbance.

Furthermore, the reflectance spectrum of RNCaSil agreed with data obtained earlier as to the optical properties the material (Part I 4.1, Table 4), which indicated a reflectance of around $90 \%$ in the visible region (measured via Tappi and ISO brightness) and overall luminescent brightness of $95.7 \%$. Therefore, no more than $5 \%$ of incidence light was absorbed by RNCaSil. That some absorbance by the NCaSils occurred was supported by the slightly higher potentials observed for NCaSil-containing cells. However, in most cases, the light absorbance by NCaSil and increase in potential was minimal and within the experimental error of the experiments. 


\section{FUTURE WORK}

In light of the conclusion that the use of NCaSils with the iodide/triiodide forms soggy sand electrolytes, any future work should explore this further. There is much potential in looking at the use of these soggy sand electrolytes in DSSCs, for example, with $\mathrm{TiO}_{2}$-based cells. As it is desirable for DSSCs to be as solid-like as possible, as well as testing with the powder/liquid samples, the cells should be tested with the gel forms of NCaSil, particularly the treated NCaSils (cerium and vanadium), which proved very promising. As the gels are not fully solid, they should be able to interpenetrate the semiconductor's structure, to obtain good connectivity with the surface, whether dyed or not. The gellike state should offer more stability than the liquid state of the iodide/triiodide redox couple, not only because of the more solid nature of the electrolyte, but also because the stability that comes with the presence of NCaSils in the electrolyte, such as the suppression of photobleaching (Part IV 1.1.5).

In investigating the use of gel NCaSils, more work could be done with regards to the formation of the gels themselves. For example, one could look at how dry each gel should be to achieve peak performance, which is expected to be different depending on the starting NCaSil. Whether gel formation could be made faster by bubbling air or oxygen through the sample could also be assessed.

As there have been promising reports of the use of ionic liquids with $\mathrm{SiO}_{2}$, electrolytes comprised of NCaSils with an iodide-based ionic liquid (such as a 1,3-dialkyimidazolium iodide) could be explored. The iodide/triiodide may be able to pass through the larger pores of NCaSils, which are usually around $200 \mathrm{~nm}$ in diameter. ${ }^{[13]}$ The resulting cells could prove very stable, using a solid electrolyte. However, achieving good connectivity with the semiconducting material's surface could prove problematic.

Finally, cell testing should be examined in ITO PET, due to the flexibility that such electrodes offer. If the soggy sand electrolytes utilised are comprised of the NCaSil gels or ionic liquid/NCaSil mixtures, lamination of the cells should be investigated.

If the DSSCs constructed from NCaSil-containing soggy sand electrolytes prove to be less efficient than cells constructed from comparative materials, it should be borne in mind NCaSil has a low production cost (of about a US\$1 per $\mathrm{kg}$ ) and facile manufacture. This could mean that, if its derived cells were found to have comparably lower conversion efficiencies, this could be outweighed if one considered efficiency to be a balance between light conversion and cost per percentage of that conversion. NCaSil-containing cells are likely to have a very high per dollar conversion efficiency. 


\section{CONCLUSIONS}

The results obtained throughout this project undoubtedly indicated that NCaSil-containing cells were dominated by the iodide/triiodide electrolyte's photogalvanic process. This was supported by the similarity between the electrolyte alone and NCaSil-containing cells with regards to the linear currentover-voltage curves, UV-vis analysis, cell performance at different wavelengths and the fact that there were only small increases in performance observed upon adding the NCaSils to the electrolyte. As such, the NCaSils were not acting as semiconducting materials, either in the capacity of DSSCs or semiconducting photogalvanic cells. However, the cells were not behaving as simple thin-layerelectrolyte photogalvanic cells either, as indicated by the complete indifference shown to change in electrode distance (where some decrease in performance was expected on increasing the distance), the different power output curve shape upon filtering out certain wavelengths of light and the increase in cell performance on using the iodide/triiodide electrolyte in conjunction with NCaSil. This suggested that the NCaSils were acting as insulating/network materials, when used with the iodide/triiodide electrolyte, to form soggy sand electrolytes.

That the NCaSils were acting synergistically with the electrolyte was supported by indications of the importance of the silicate backbone to cell performance. Tests where $\mathrm{CaI}_{2}$ was investigated showed lower performances, as did samples of iodide/triiodide with the equivalent amount of $\mathrm{Ca}^{2+}$ added as would be present in an RNCaSil-containing cell. Furthermore, testing with synthetic silicon oil gels was unsuccessful. XPS performed on the more highly efficient g-NCaSil, formed from RNCaSil and iodide/triiodide (70 wt \% ethanol $30 \mathrm{wt} \%$ water) showed the importance of the binding of iodine to $\mathrm{Ca}^{2+}$ and silicon.

The NCaSil cells were compared to two materials, which had been documented as insulating materials, $\mathrm{TiO}_{2}$ and $\mathrm{SiO}_{2}$. RNCaSil was the only non-metal-treated NCaSil that exhibited comparative cell performances. However, Ce- and V-NCaSil exhibited very promising results, with currents over double that of the equivalent $\mathrm{TiO}_{2}$ and $\mathrm{SiO}_{2}$ cells.

Free from any $\mathrm{Ca}^{2+}$ additive, the determining feature of cell performance was the $\mathrm{Ca}^{2+}$ accessibility of the NCaSils and, thus, surface area. This was attributed to the increase in conductivity within the iodide/triiodide electrolyte from the influx of $\mathrm{Ca}^{2+}$ from the NCaSils, rather than any synergistic effect. However, when $5 \mathrm{wt} \% \mathrm{CaCl}_{2}$ was added to the electrolyte (water-based), the driving force for the 
removal of $\mathrm{Ca}^{2+}$ from the NCaSil surface was lowered and cell performance became dependent on the NCaSil's stability. This was because the more stable NCaSils were able to maintain more of their $\mathrm{Ca}^{2+}$, which allowed them to form soggy sand electrolytes through the interaction of iodine with surfacebound $\mathrm{Ca}^{2+}$. Thus, an increase in cell performance on the addition of the NCaSils to the electrolyte was observed. Above $5 \mathrm{wt} \% \mathrm{CaCl}_{2}$ (or $1.5 \mathrm{wt} \%$ in the $70 \mathrm{wt} \%$ ethanol-based electrolyte), $\mathrm{Ca}(\mathrm{OH})_{2}$ precipitated, due to the alkalinity of the NCaSils, which resulted in a decrease in cell current compared to the electrolyte tested alone.

The performance of NCaSil was dependent on the mixing order when forming the material; NaSil should be added to $\mathrm{Ca}(\mathrm{OH})_{2}$. This was not due to the resultant particle sizes, which were the same regardless of mixing order, but the higher $\mathrm{Ca}^{2+}$ content. NCaSils made as such were more readily able to form gels. That the higher $\mathrm{Ca}^{2+}$ content allowed for gel formation was not surprising due to the dominant binding of iodine to $\mathrm{Ca}^{2+}$ as shown by XPS and cyclic voltammetry. The NCaSils gels exhibited enhanced cell performances over their non-gel counterparts, particularly the Ce- and Vtreated g-NCaSils. Furthermore, the gels showed promise as soggy sand electrolytes in DSSCs, because of the greater stability offered by the semi-solid state over all other NCaSils investigated.

Therefore, any future work should look at using NCaSils with iodide/triiodide as soggy sand electrolytes in DSSCs. This should include both the powder/liquid samples as well as the gel forms of NCaSils, particularly the Ce- and V-g-NCaSil. The electrolytes should be solvated in $70 \mathrm{wt} \%$ ethanol $30 \mathrm{wt} \%$ water, with $1.5 \mathrm{wt} \% \mathrm{CaCl}_{2}$ added to the solution. Furthermore, the dark electrode should be backed with a highly reflective surface (such as the Teflon tape tested here), to act as a scattering surface to maximise the light path. 


\section{APPENDIX}

1 IR Spectra of RNCaSil and Differently Solvated Iodide/Triiodide

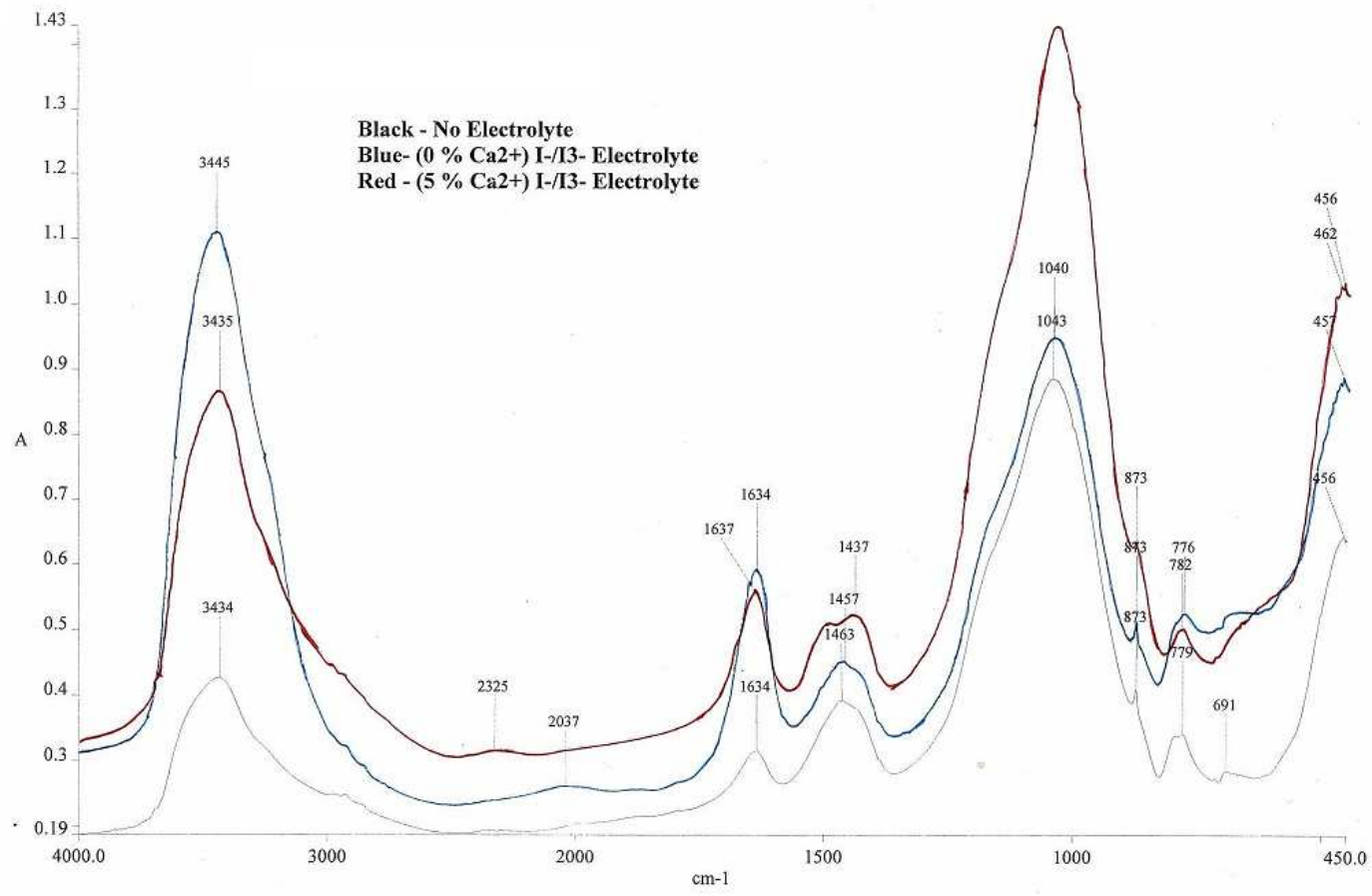

Figure 98. IR Spectrum of RNCaSil Alone and With Water-Based Iodide/Triiodide ( 0 and 5 wt $\left.\% \mathbf{C a C l}_{2}\right)$.

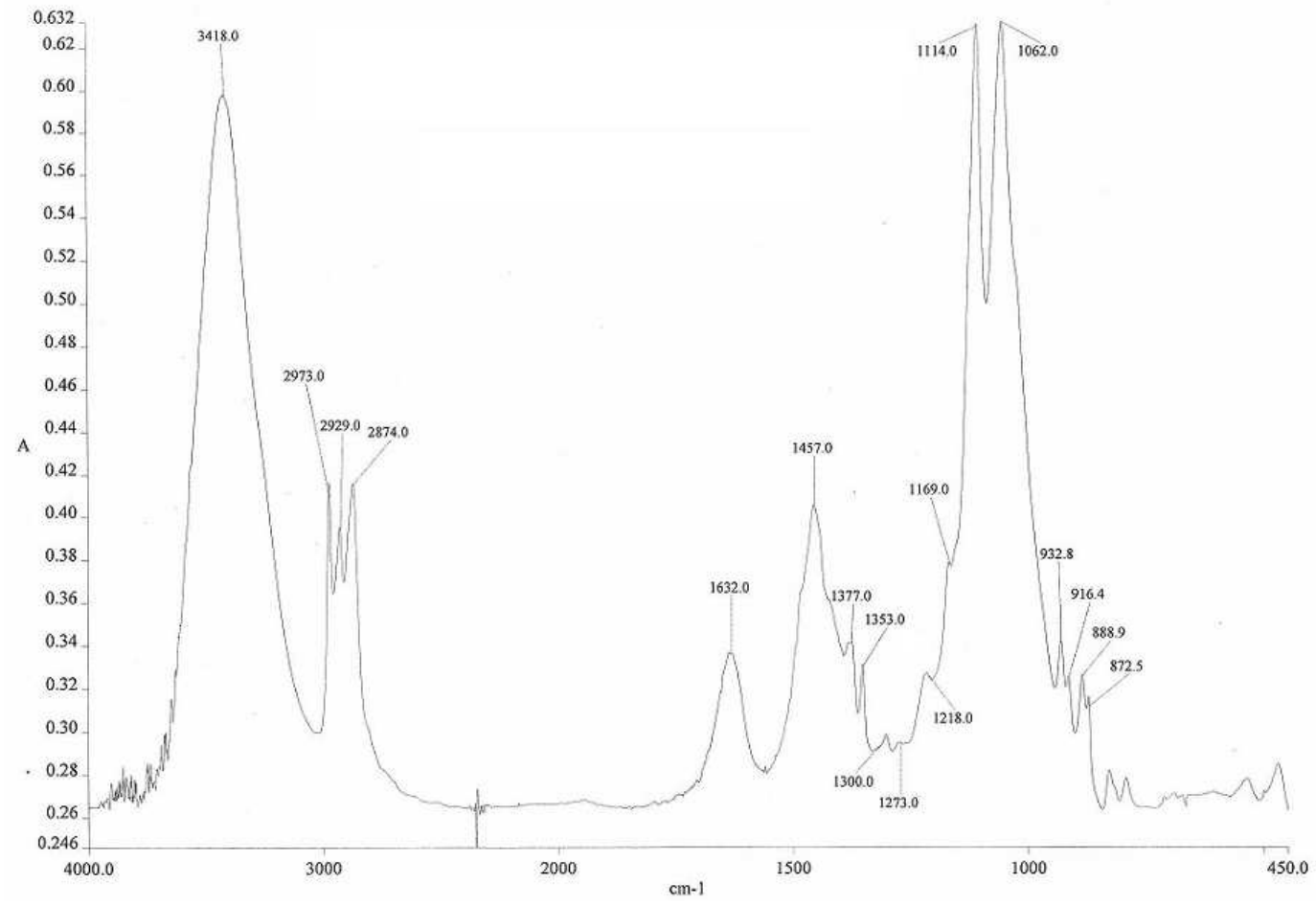

Figure 99. IR Spectrum of RNCaSil With 70 wt \% 2-Ethoxyethanol Iodide/Triiodide. 


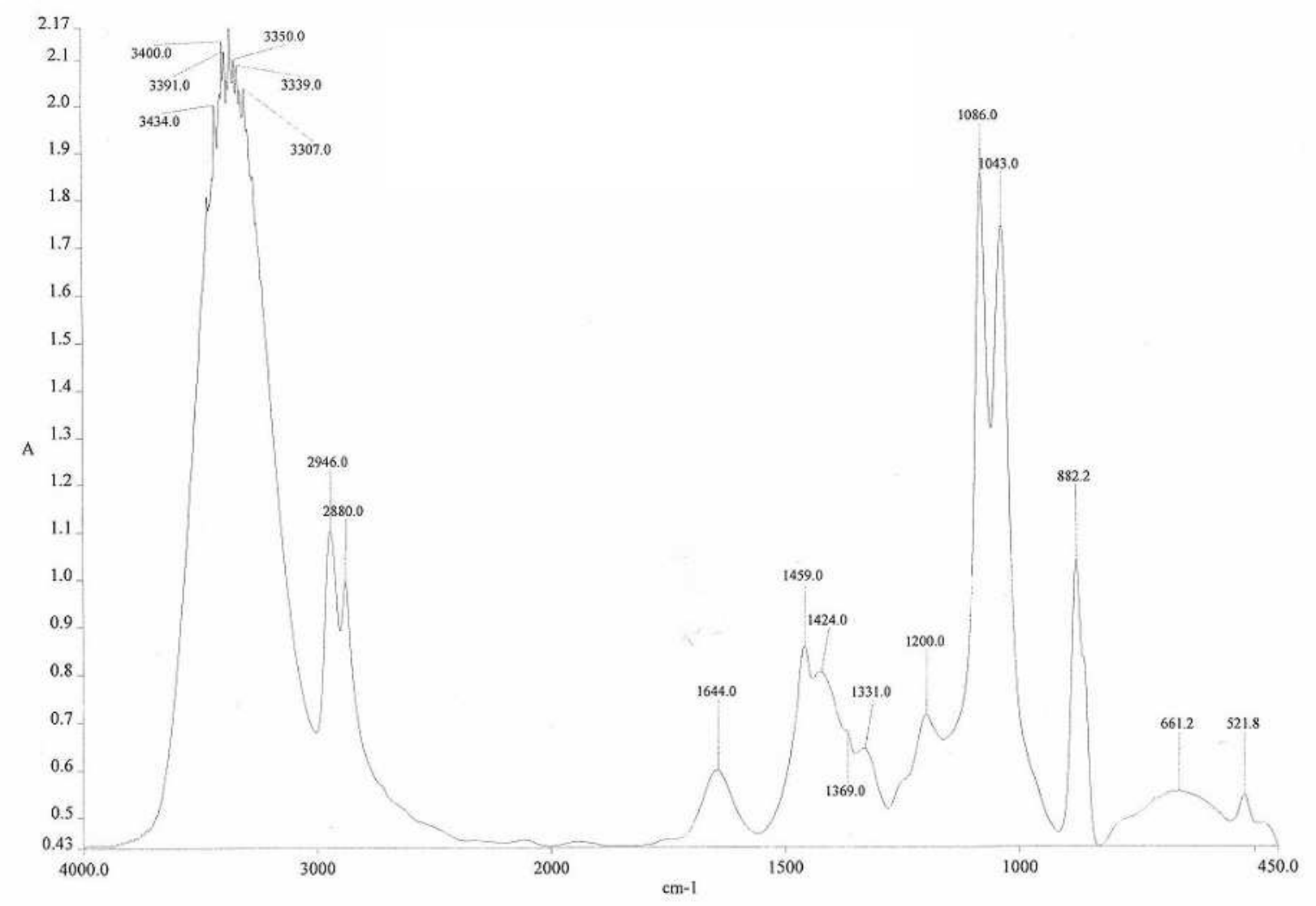

Figure 100. IR Spectrum of RNCaSil With 70 wt\% Ethylene Glycol Iodide/Triiodide.

\section{UV-vis Transmission Spectrum of Laminated Plastic and ITO PET}

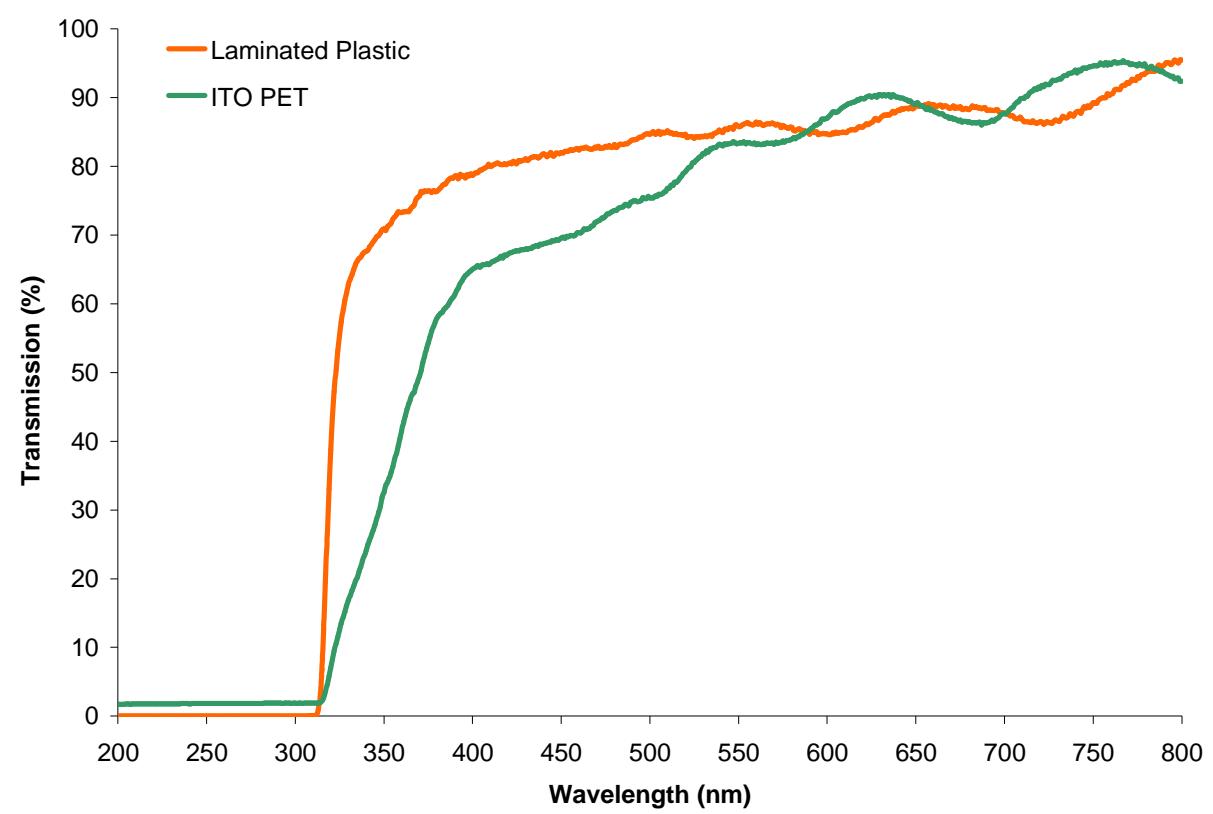

Figure 101. UV-vis Transmission Spectrum of the Laminated Plastic Used and ITO PET. 


\section{$3 \quad{ }^{1} \mathrm{H}$ and ${ }^{13} \mathrm{C}$ NMR Spectra of g-NCaSil}

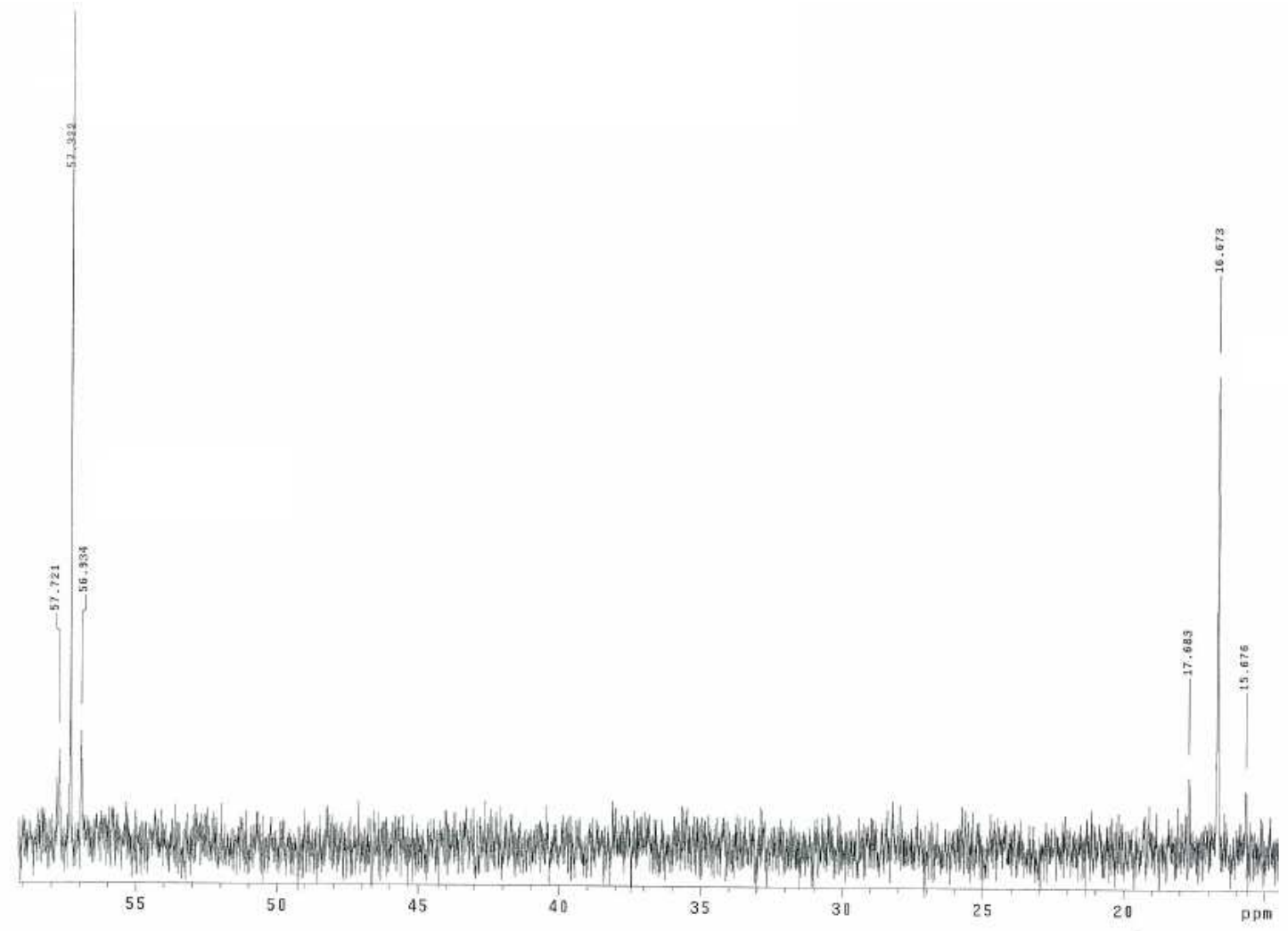

Figure 102. ${ }^{13} \mathrm{C}$ NMR Spectrum of g-NCaSil after 72 hours Illumination.

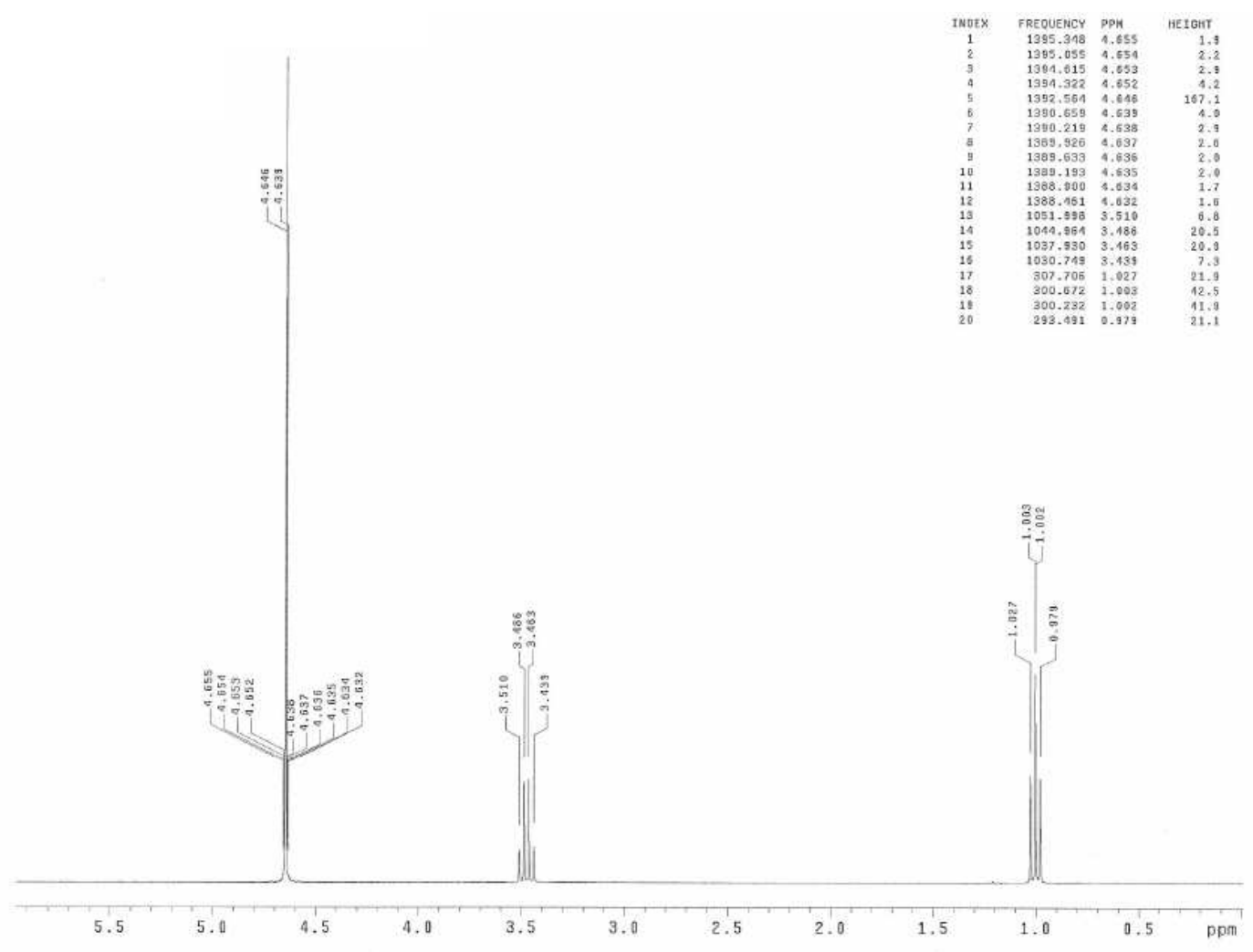

Figure 103. ${ }^{1} \mathrm{H}$ NMR Spectrum of g-NCaSil after 72 hours Illumination. 


\section{$4 \quad$ IR Spectrum of $\mathrm{g}-\mathrm{NCaSil}$}

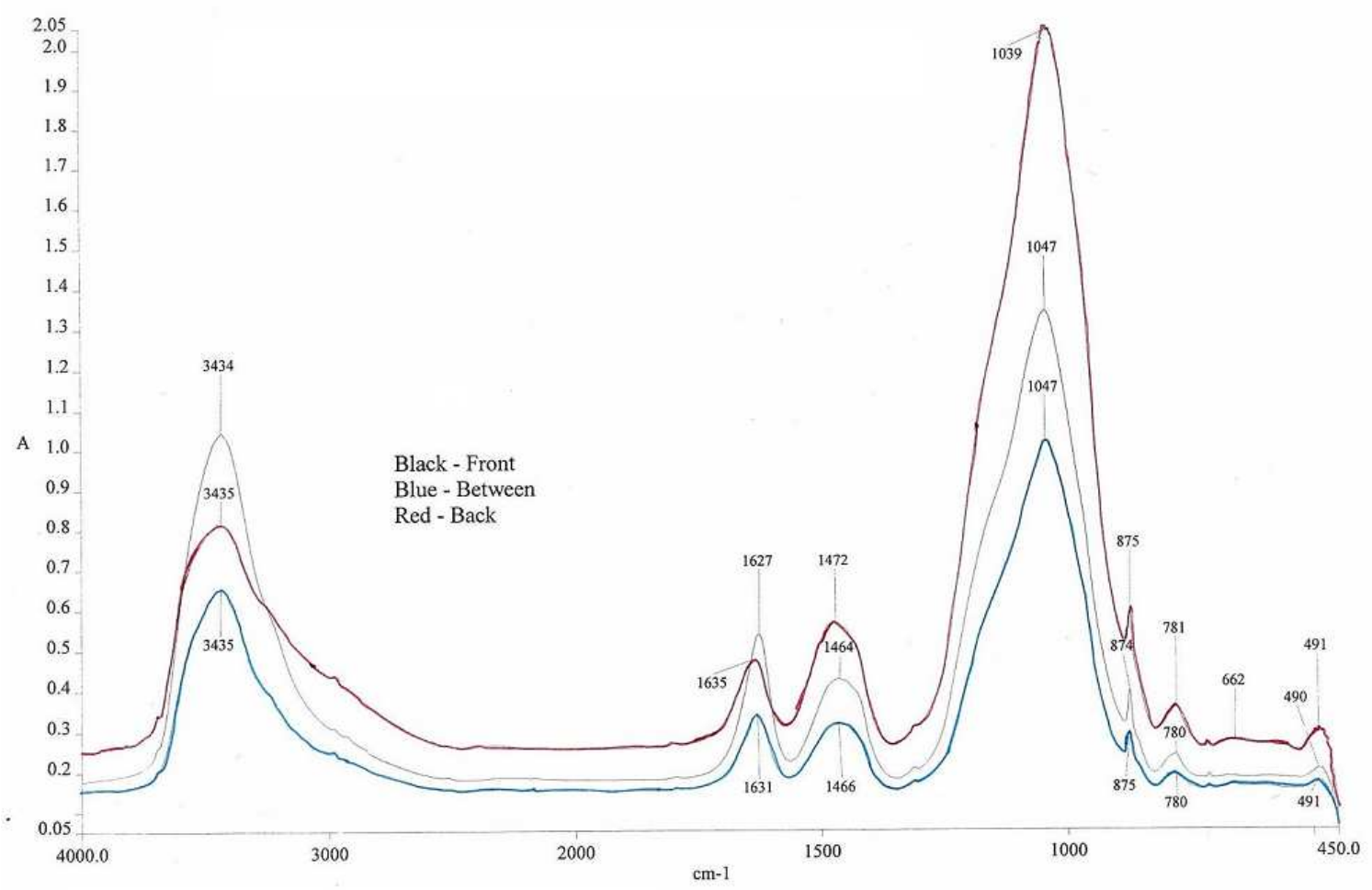

Figure 104. IR Spectra of g-NCaSil after 7 Days Illumination: The Sample in Front of the Photo electrode, Between the Electrodes and Behind the Back Electrode.

\section{$5 \quad$ Gas IR Spectrum of Gas from TEOS Gel Formation}

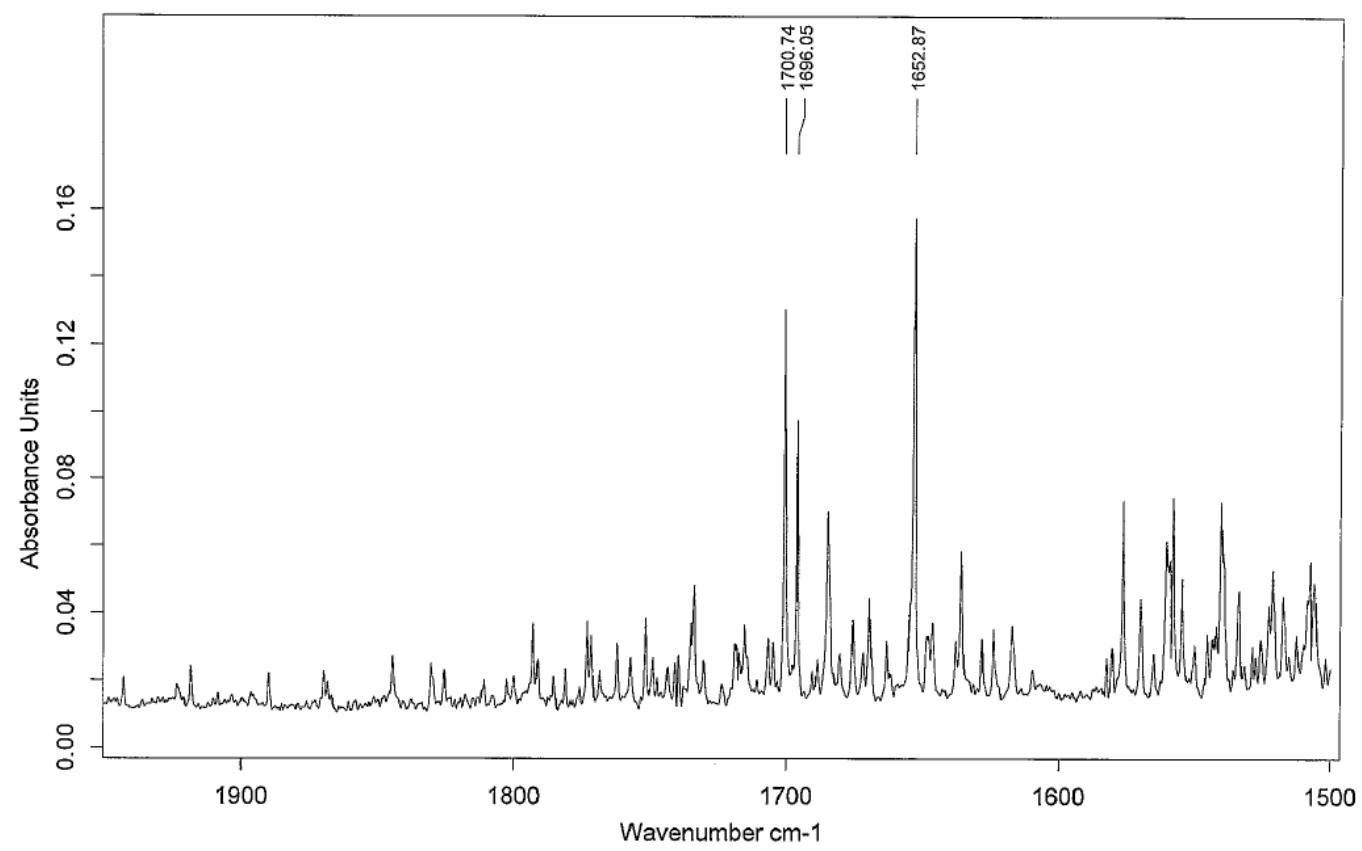

Figure 105. Gas IR Spectrum of Gas Produced from the Formation of TEOS Gels. 


\section{$6 \quad$ XRD of g-NCaSil}

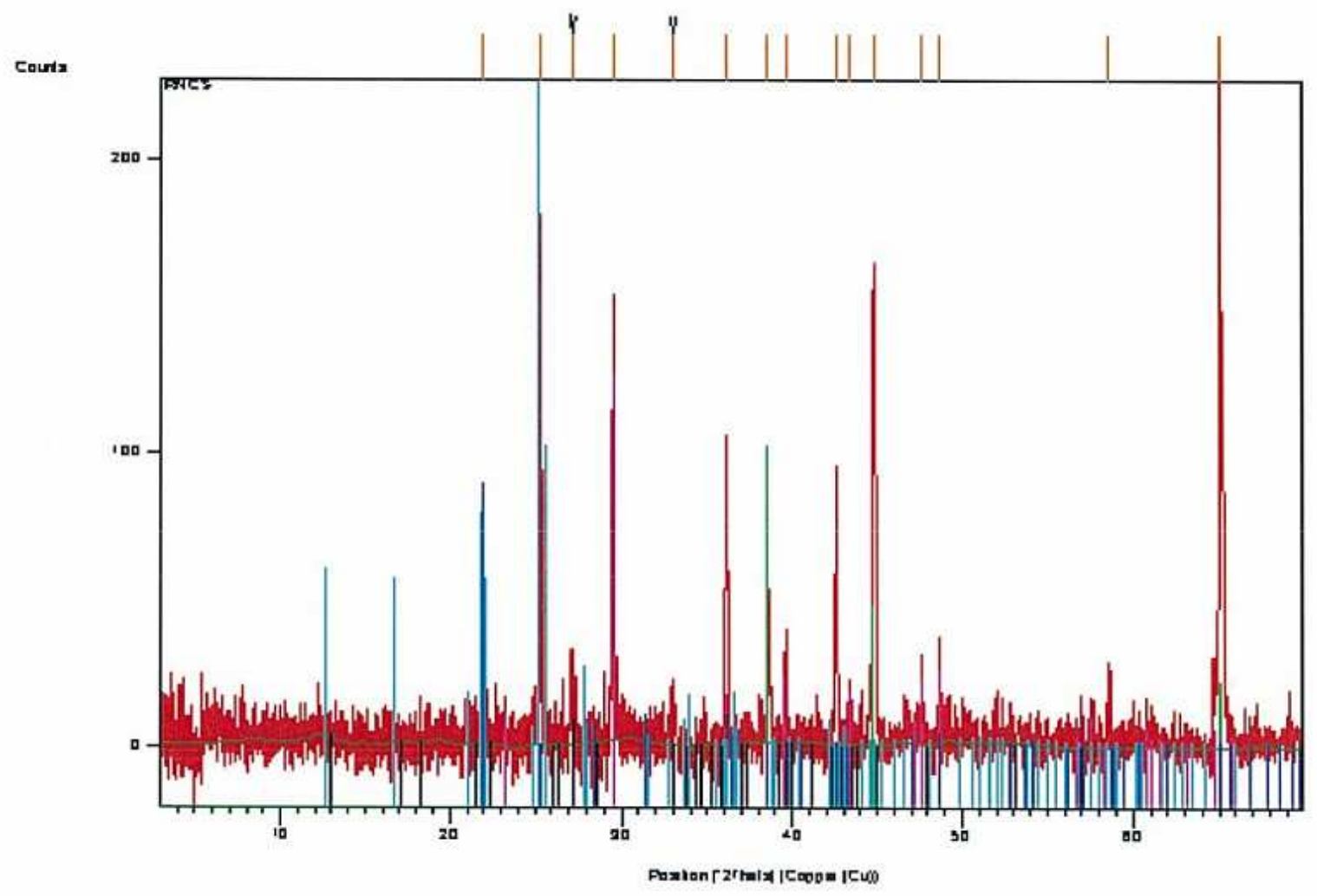

Figure 106. XRD Spectrum of Dried g-NCaSil.

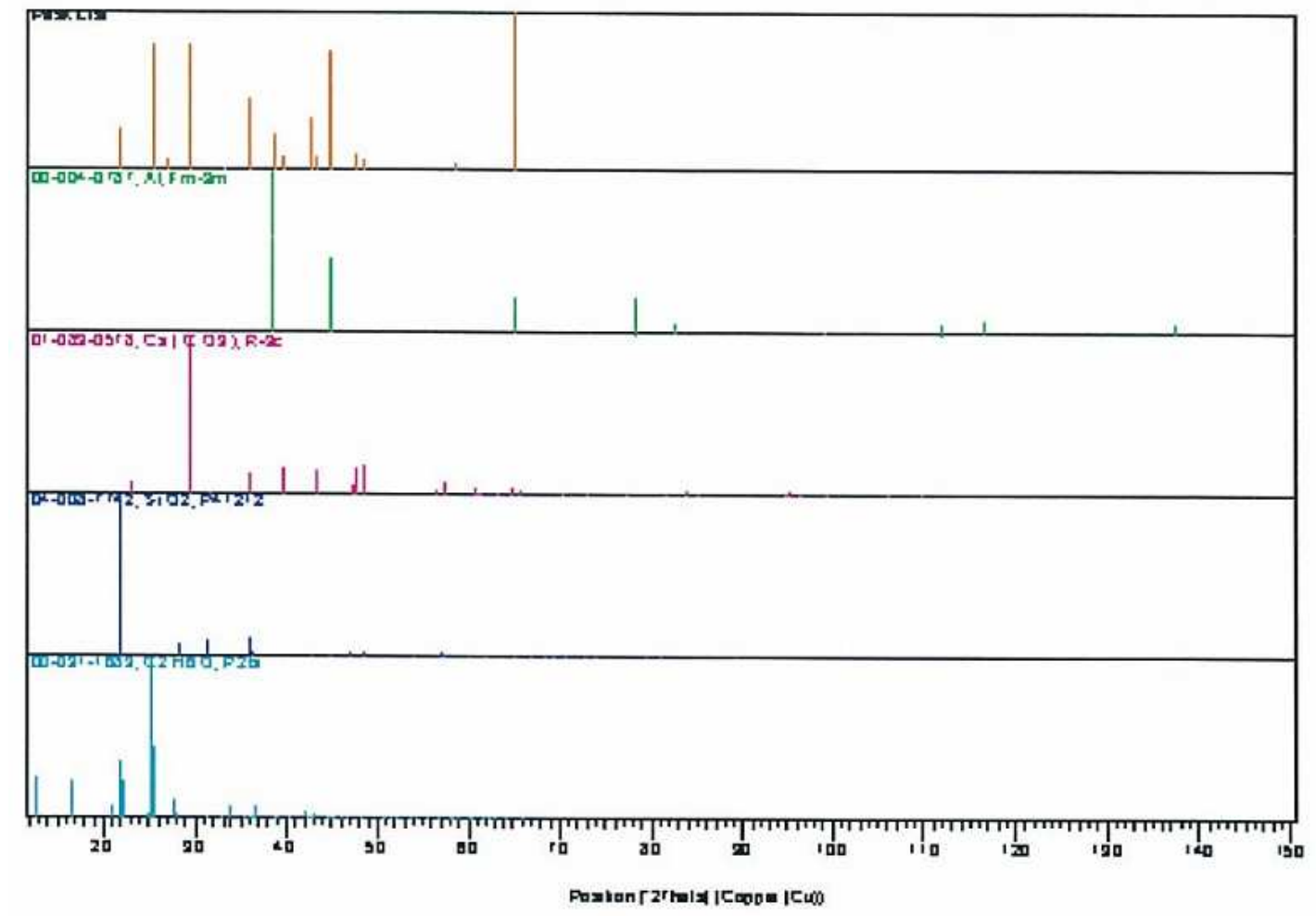

Figure 107. XRD Spectrum Graphic Analysis of Dried g-NCaSil. 


\section{$7 \quad$ Solubility of $\mathrm{Ca}^{2+}$ at Different pHs}

$\mathrm{Ca}(\mathrm{OH})_{2(\mathrm{~s})} \leftrightarrow \mathrm{Ca}^{2+}+2 \mathrm{OH}^{-}$

$\mathrm{K}_{\mathrm{sp}}=\left[\mathrm{Ca}^{2+}\right]\left[\mathrm{OH}^{-}\right]^{2}=6.4 \times 10^{-6} \mathrm{~mol} / \mathrm{dm}^{3}$

$[\mathrm{OH}]=10^{-(14-\mathrm{pOH})}$

Table 9. Solubility of $\mathrm{Ca}^{2+}$ Dependent on the Concentration of $\mathrm{OH}$.

\begin{tabular}{|c|c|c|c|}
\hline $\mathbf{p H}$ & {$[\mathbf{O H}]$} & {$[\mathbf{O H}]^{2}$} & $\left.{ }^{2} \mathbf{C a}^{2+}\right]$ \\
\hline 0.0 & $1.00 \mathrm{E}-14$ & $1.00 \mathrm{E}-28$ & $6.40 \mathrm{E}+22$ \\
\hline 0.1 & $1.26 \mathrm{E}-14$ & $1.58 \mathrm{E}-28$ & $4.04 \mathrm{E}+22$ \\
\hline 0.2 & $1.58 \mathrm{E}-14$ & $2.51 \mathrm{E}-28$ & $2.55 \mathrm{E}+22$ \\
\hline 0.3 & $2.00 \mathrm{E}-14$ & $3.98 \mathrm{E}-28$ & $1.61 \mathrm{E}+22$ \\
\hline 0.4 & $2.51 \mathrm{E}-14$ & $6.31 \mathrm{E}-28$ & $1.01 \mathrm{E}+22$ \\
\hline 0.5 & $3.16 \mathrm{E}-14$ & $1.00 \mathrm{E}-27$ & $6.40 \mathrm{E}+21$ \\
\hline 0.6 & $3.98 \mathrm{E}-14$ & $1.58 \mathrm{E}-27$ & $4.04 \mathrm{E}+21$ \\
\hline 0.7 & $5.01 \mathrm{E}-14$ & $2.51 \mathrm{E}-27$ & $2.55 \mathrm{E}+21$ \\
\hline 0.8 & $6.31 \mathrm{E}-14$ & $3.98 \mathrm{E}-27$ & $1.61 \mathrm{E}+21$ \\
\hline 0.9 & $7.94 \mathrm{E}-14$ & $6.31 \mathrm{E}-27$ & $1.01 \mathrm{E}+21$ \\
\hline 1.0 & $1.00 \mathrm{E}-13$ & $1.00 \mathrm{E}-26$ & $6.40 \mathrm{E}+20$ \\
\hline 1.1 & $1.26 \mathrm{E}-13$ & $1.58 \mathrm{E}-26$ & $4.04 \mathrm{E}+20$ \\
\hline 1.2 & $1.58 \mathrm{E}-13$ & $2.51 \mathrm{E}-26$ & $2.55 \mathrm{E}+20$ \\
\hline 1.3 & $2.00 \mathrm{E}-13$ & $3.98 \mathrm{E}-26$ & $1.61 \mathrm{E}+20$ \\
\hline 1.4 & $2.51 \mathrm{E}-13$ & $6.31 \mathrm{E}-26$ & $1.01 \mathrm{E}+20$ \\
\hline 1.5 & $3.16 \mathrm{E}-13$ & $1.00 \mathrm{E}-25$ & $6.40 \mathrm{E}+19$ \\
\hline 1.6 & $3.98 \mathrm{E}-13$ & $1.58 \mathrm{E}-25$ & $4.04 \mathrm{E}+19$ \\
\hline 1.7 & $5.01 \mathrm{E}-13$ & $2.51 \mathrm{E}-25$ & $2.55 \mathrm{E}+19$ \\
\hline 1.8 & $6.31 \mathrm{E}-13$ & $3.98 \mathrm{E}-25$ & $1.61 \mathrm{E}+19$ \\
\hline 1.9 & $7.94 \mathrm{E}-13$ & $6.31 \mathrm{E}-25$ & $1.01 \mathrm{E}+19$ \\
\hline 2.0 & $1.00 \mathrm{E}-12$ & $1.00 \mathrm{E}-24$ & $6.40 \mathrm{E}+18$ \\
\hline 2.1 & $1.26 \mathrm{E}-12$ & $1.58 \mathrm{E}-24$ & $4.04 \mathrm{E}+18$ \\
\hline 2.2 & $1.58 \mathrm{E}-12$ & $2.51 \mathrm{E}-24$ & $2.55 \mathrm{E}+18$ \\
\hline 2.3 & $2.00 \mathrm{E}-12$ & $3.98 \mathrm{E}-24$ & $1.61 \mathrm{E}+18$ \\
\hline 2.4 & $2.51 \mathrm{E}-12$ & $6.31 \mathrm{E}-24$ & $1.01 \mathrm{E}+18$ \\
\hline 2.5 & $3.16 \mathrm{E}-12$ & $1.00 \mathrm{E}-23$ & $6.40 \mathrm{E}+17$ \\
\hline 2.6 & $3.98 \mathrm{E}-12$ & $1.58 \mathrm{E}-23$ & $4.04 \mathrm{E}+17$ \\
\hline 2.7 & $5.01 \mathrm{E}-12$ & $2.51 \mathrm{E}-23$ & $2.55 \mathrm{E}+17$ \\
\hline 2.8 & $6.31 \mathrm{E}-12$ & $3.98 \mathrm{E}-23$ & $1.61 \mathrm{E}+17$ \\
\hline 2.9 & $7.94 \mathrm{E}-12$ & $6.31 \mathrm{E}-23$ & $1.01 \mathrm{E}+17$ \\
\hline 3.0 & $1.00 \mathrm{E}-11$ & $1.00 \mathrm{E}-22$ & $6.40 \mathrm{E}+16$ \\
\hline 3.1 & $1.26 \mathrm{E}-11$ & $1.58 \mathrm{E}-22$ & $4.04 \mathrm{E}+16$ \\
\hline 3.2 & $1.58 \mathrm{E}-11$ & $2.51 \mathrm{E}-22$ & $2.55 \mathrm{E}+16$ \\
\hline 3.3 & $2.00 \mathrm{E}-11$ & $3.98 \mathrm{E}-22$ & $1.61 \mathrm{E}+16$ \\
\hline 3.4 & $2.51 \mathrm{E}-11$ & $6.31 \mathrm{E}-22$ & $1.01 \mathrm{E}+16$ \\
\hline 3.5 & $3.16 \mathrm{E}-11$ & $1.00 \mathrm{E}-21$ & $6.40 \mathrm{E}+15$ \\
\hline 3.6 & $3.98 \mathrm{E}-11$ & $1.58 \mathrm{E}-21$ & $4.04 \mathrm{E}+15$ \\
\hline 3.7 & $5.01 \mathrm{E}-11$ & $2.51 \mathrm{E}-21$ & $2.55 \mathrm{E}+15$ \\
\hline 3.8 & $6.31 \mathrm{E}-11$ & $3.98 \mathrm{E}-21$ & $1.61 \mathrm{E}+15$ \\
\hline 3.9 & $7.94 \mathrm{E}-11$ & $6.31 \mathrm{E}-21$ & $1.01 \mathrm{E}+15$ \\
\hline 4.0 & $1.00 \mathrm{E}-10$ & $1.00 \mathrm{E}-20$ & $6.40 \mathrm{E}+14$ \\
\hline
\end{tabular}

\begin{tabular}{|c|c|c|c|}
\hline $\mathbf{p H}$ & {$[\mathbf{O H}]$} & {$[\mathbf{O H}]^{2}$} & {$\left[\mathbf{C a}^{2+}\right]$} \\
\hline 4.1 & $1.26 \mathrm{E}-10$ & $1.58 \mathrm{E}-20$ & $4.04 \mathrm{E}+14$ \\
\hline 4.2 & $1.58 \mathrm{E}-10$ & $2.51 \mathrm{E}-20$ & $2.55 \mathrm{E}+14$ \\
\hline 4.3 & $2.00 \mathrm{E}-10$ & $3.98 \mathrm{E}-20$ & $1.61 \mathrm{E}+14$ \\
\hline 4.4 & $2.51 \mathrm{E}-10$ & $6.31 \mathrm{E}-20$ & $1.01 \mathrm{E}+14$ \\
\hline 4.5 & $3.16 \mathrm{E}-10$ & $1.00 \mathrm{E}-19$ & $6.40 \mathrm{E}+13$ \\
\hline 4.6 & $3.98 \mathrm{E}-10$ & $1.58 \mathrm{E}-19$ & $4.04 \mathrm{E}+13$ \\
\hline 4.7 & $5.01 \mathrm{E}-10$ & $2.51 \mathrm{E}-19$ & $2.55 \mathrm{E}+13$ \\
\hline 4.8 & $6.31 \mathrm{E}-10$ & $3.98 \mathrm{E}-19$ & $1.61 \mathrm{E}+13$ \\
\hline 4.9 & $7.94 \mathrm{E}-10$ & $6.31 \mathrm{E}-19$ & $1.01 \mathrm{E}+13$ \\
\hline 5.0 & $1.00 \mathrm{E}-09$ & $1.00 \mathrm{E}-18$ & $6.40 \mathrm{E}+12$ \\
\hline 5.1 & $1.26 \mathrm{E}-09$ & $1.58 \mathrm{E}-18$ & $4.04 \mathrm{E}+12$ \\
\hline 5.2 & $1.58 \mathrm{E}-09$ & $2.51 \mathrm{E}-18$ & $2.55 \mathrm{E}+12$ \\
\hline 5.3 & $2.00 \mathrm{E}-09$ & $3.98 \mathrm{E}-18$ & $1.61 \mathrm{E}+12$ \\
\hline 5.4 & $2.51 \mathrm{E}-09$ & $6.31 \mathrm{E}-18$ & $1.01 \mathrm{E}+12$ \\
\hline 5.5 & $3.16 \mathrm{E}-09$ & $1.00 \mathrm{E}-17$ & $6.40 \mathrm{E}+11$ \\
\hline 5.6 & $3.98 \mathrm{E}-09$ & $1.58 \mathrm{E}-17$ & $4.04 \mathrm{E}+11$ \\
\hline 5.7 & $5.01 \mathrm{E}-09$ & $2.51 \mathrm{E}-17$ & $2.55 \mathrm{E}+11$ \\
\hline 5.8 & $6.31 \mathrm{E}-09$ & $3.98 \mathrm{E}-17$ & $1.61 \mathrm{E}+11$ \\
\hline 5.9 & $7.94 \mathrm{E}-09$ & $6.31 \mathrm{E}-17$ & $1.01 \mathrm{E}+11$ \\
\hline 6.0 & $1.00 \mathrm{E}-08$ & $1.00 \mathrm{E}-16$ & $6.40 \mathrm{E}+10$ \\
\hline 6.1 & $1.26 \mathrm{E}-08$ & $1.58 \mathrm{E}-16$ & $4.04 \mathrm{E}+10$ \\
\hline 6.2 & $1.58 \mathrm{E}-08$ & $2.51 \mathrm{E}-16$ & $2.55 \mathrm{E}+10$ \\
\hline 6.3 & $2.00 \mathrm{E}-08$ & $3.98 \mathrm{E}-16$ & $1.61 \mathrm{E}+10$ \\
\hline 6.4 & $2.51 \mathrm{E}-08$ & $6.31 \mathrm{E}-16$ & $1.01 \mathrm{E}+10$ \\
\hline 6.5 & $3.16 \mathrm{E}-08$ & $1.00 \mathrm{E}-15$ & $6.40 \mathrm{E}+09$ \\
\hline 6.6 & $3.98 \mathrm{E}-08$ & $1.58 \mathrm{E}-15$ & $4.04 \mathrm{E}+09$ \\
\hline 6.7 & $5.01 \mathrm{E}-08$ & $2.51 \mathrm{E}-15$ & $2.55 \mathrm{E}+09$ \\
\hline 6.8 & $6.31 \mathrm{E}-08$ & $3.98 \mathrm{E}-15$ & $1.61 \mathrm{E}+09$ \\
\hline 6.9 & $7.94 \mathrm{E}-08$ & $6.31 \mathrm{E}-15$ & $1.01 \mathrm{E}+09$ \\
\hline 7.0 & $1.00 \mathrm{E}-07$ & $1.00 \mathrm{E}-14$ & $6.40 \mathrm{E}+08$ \\
\hline 7.1 & $1.26 \mathrm{E}-07$ & $1.58 \mathrm{E}-14$ & $4.04 \mathrm{E}+08$ \\
\hline 7.2 & $1.58 \mathrm{E}-07$ & $2.51 \mathrm{E}-14$ & $2.55 \mathrm{E}+08$ \\
\hline 7.3 & $2.00 \mathrm{E}-07$ & $3.98 \mathrm{E}-14$ & $1.61 \mathrm{E}+08$ \\
\hline 7.4 & $2.51 \mathrm{E}-07$ & $6.31 \mathrm{E}-14$ & $1.01 \mathrm{E}+08$ \\
\hline 7.5 & $3.16 \mathrm{E}-07$ & $1.00 \mathrm{E}-13$ & $6.40 \mathrm{E}+07$ \\
\hline 7.6 & $3.98 \mathrm{E}-07$ & $1.58 \mathrm{E}-13$ & $4.04 \mathrm{E}+07$ \\
\hline 7.7 & $5.01 \mathrm{E}-07$ & $2.51 \mathrm{E}-13$ & $2.55 \mathrm{E}+07$ \\
\hline 7.8 & $6.31 \mathrm{E}-07$ & $3.98 \mathrm{E}-13$ & $1.61 \mathrm{E}+07$ \\
\hline 7.9 & $7.94 \mathrm{E}-07$ & $6.31 \mathrm{E}-13$ & $1.01 \mathrm{E}+07$ \\
\hline 8.0 & $1.00 \mathrm{E}-06$ & $1.00 \mathrm{E}-12$ & $6.40 \mathrm{E}+06$ \\
\hline 8.1 & $1.26 \mathrm{E}-06$ & $1.58 \mathrm{E}-12$ & $4.04 \mathrm{E}+06$ \\
\hline & & & \\
\hline
\end{tabular}




\begin{tabular}{|c|c|c|c|}
\hline $\mathbf{p H}$ & {$[\mathbf{O H}]$} & {$[\mathbf{O H}]^{2}$} & {$\left[\mathbf{C a}^{2+}\right]$} \\
\hline 8.2 & $1.58 \mathrm{E}-06$ & $2.51 \mathrm{E}-12$ & $2.55 \mathrm{E}+06$ \\
\hline 8.3 & $2.00 \mathrm{E}-06$ & $3.98 \mathrm{E}-12$ & $1.61 \mathrm{E}+06$ \\
\hline 8.4 & $2.51 \mathrm{E}-06$ & $6.31 \mathrm{E}-12$ & $1.01 \mathrm{E}+06$ \\
\hline 8.5 & $3.16 \mathrm{E}-06$ & $1.00 \mathrm{E}-11$ & $6.40 \mathrm{E}+05$ \\
\hline 8.6 & $3.98 \mathrm{E}-06$ & $1.58 \mathrm{E}-11$ & $4.04 \mathrm{E}+05$ \\
\hline 8.7 & $5.01 \mathrm{E}-06$ & $2.51 \mathrm{E}-11$ & $2.55 \mathrm{E}+05$ \\
\hline 8.8 & $6.31 \mathrm{E}-06$ & $3.98 \mathrm{E}-11$ & $1.61 \mathrm{E}+05$ \\
\hline 8.9 & $7.94 \mathrm{E}-06$ & $6.31 \mathrm{E}-11$ & $1.01 \mathrm{E}+05$ \\
\hline 9.0 & $1.00 \mathrm{E}-05$ & $1.00 \mathrm{E}-10$ & $6.40 \mathrm{E}+04$ \\
\hline 9.1 & $1.26 \mathrm{E}-05$ & $1.58 \mathrm{E}-10$ & $4.04 \mathrm{E}+04$ \\
\hline 9.2 & $1.58 \mathrm{E}-05$ & $2.51 \mathrm{E}-10$ & $2.55 \mathrm{E}+04$ \\
\hline 9.3 & $2.00 \mathrm{E}-05$ & $3.98 \mathrm{E}-10$ & $1.61 \mathrm{E}+04$ \\
\hline 9.4 & $2.51 \mathrm{E}-05$ & $6.31 \mathrm{E}-10$ & $1.01 \mathrm{E}+04$ \\
\hline 9.5 & $3.16 \mathrm{E}-05$ & $1.00 \mathrm{E}-09$ & $6.40 \mathrm{E}+03$ \\
\hline 9.6 & $3.98 \mathrm{E}-05$ & $1.58 \mathrm{E}-09$ & $4.04 \mathrm{E}+03$ \\
\hline 9.7 & $5.01 \mathrm{E}-05$ & $2.51 \mathrm{E}-09$ & $2.55 \mathrm{E}+03$ \\
\hline 9.8 & $6.31 \mathrm{E}-05$ & $3.98 \mathrm{E}-09$ & $1.61 \mathrm{E}+03$ \\
\hline 9.9 & $7.94 \mathrm{E}-05$ & $6.31 \mathrm{E}-09$ & $1.01 \mathrm{E}+03$ \\
\hline 10.0 & $1.00 \mathrm{E}-04$ & $1.00 \mathrm{E}-08$ & $6.40 \mathrm{E}+02$ \\
\hline 10.1 & $1.26 \mathrm{E}-04$ & $1.58 \mathrm{E}-08$ & $4.04 \mathrm{E}+02$ \\
\hline 10.2 & $1.58 \mathrm{E}-04$ & $2.51 \mathrm{E}-08$ & $2.55 \mathrm{E}+02$ \\
\hline 10.3 & $2.00 \mathrm{E}-04$ & $3.98 \mathrm{E}-08$ & $1.61 \mathrm{E}+02$ \\
\hline 10.4 & $2.51 \mathrm{E}-04$ & $6.31 \mathrm{E}-08$ & $1.01 \mathrm{E}+02$ \\
\hline 10.5 & $3.16 \mathrm{E}-04$ & $1.00 \mathrm{E}-07$ & $6.40 \mathrm{E}+01$ \\
\hline 10.6 & $3.98 \mathrm{E}-04$ & $1.58 \mathrm{E}-07$ & $4.04 \mathrm{E}+01$ \\
\hline 10.7 & $5.01 \mathrm{E}-04$ & $2.51 \mathrm{E}-07$ & $2.55 \mathrm{E}+01$ \\
\hline 10.8 & $6.31 \mathrm{E}-04$ & $3.98 \mathrm{E}-07$ & $1.61 \mathrm{E}+01$ \\
\hline 10.9 & $7.94 \mathrm{E}-04$ & $6.31 \mathrm{E}-07$ & $1.01 \mathrm{E}+01$ \\
\hline 11.0 & $1.00 \mathrm{E}-03$ & $1.00 \mathrm{E}-06$ & $6.40 \mathrm{E}+00$ \\
\hline 11.1 & $1.26 \mathrm{E}-03$ & $1.58 \mathrm{E}-06$ & $4.04 \mathrm{E}+00$ \\
\hline
\end{tabular}

\begin{tabular}{|c|c|c|c|}
\hline $\mathbf{p H}$ & {$[\mathbf{O H}]$} & {$[\mathbf{O H}]^{2}$} & {$\left[\mathbf{C a}^{2+}\right]$} \\
\hline 11.2 & $1.58 \mathrm{E}-03$ & $2.51 \mathrm{E}-06$ & $2.55 \mathrm{E}+00$ \\
\hline 11.3 & $2.00 \mathrm{E}-03$ & $3.98 \mathrm{E}-06$ & $1.61 \mathrm{E}+00$ \\
\hline 11.4 & $2.51 \mathrm{E}-03$ & $6.31 \mathrm{E}-06$ & $1.01 \mathrm{E}+00$ \\
\hline 11.5 & $3.16 \mathrm{E}-03$ & $1.00 \mathrm{E}-05$ & $6.40 \mathrm{E}-01$ \\
\hline 11.6 & $3.98 \mathrm{E}-03$ & $1.58 \mathrm{E}-05$ & $4.04 \mathrm{E}-01$ \\
\hline 11.7 & $5.01 \mathrm{E}-03$ & $2.51 \mathrm{E}-05$ & $2.55 \mathrm{E}-01$ \\
\hline 11.8 & $6.31 \mathrm{E}-03$ & $3.98 \mathrm{E}-05$ & $1.61 \mathrm{E}-01$ \\
\hline 11.9 & $7.94 \mathrm{E}-03$ & $6.31 \mathrm{E}-05$ & $1.01 \mathrm{E}-01$ \\
\hline 12.0 & $1.00 \mathrm{E}-02$ & $1.00 \mathrm{E}-04$ & $6.40 \mathrm{E}-02$ \\
\hline 12.1 & $1.26 \mathrm{E}-02$ & $1.58 \mathrm{E}-04$ & $4.04 \mathrm{E}-02$ \\
\hline 12.2 & $1.58 \mathrm{E}-02$ & $2.51 \mathrm{E}-04$ & $2.55 \mathrm{E}-02$ \\
\hline 12.3 & $2.00 \mathrm{E}-02$ & $3.98 \mathrm{E}-04$ & $1.61 \mathrm{E}-02$ \\
\hline 12.4 & $2.51 \mathrm{E}-02$ & $6.31 \mathrm{E}-04$ & $1.01 \mathrm{E}-02$ \\
\hline 12.5 & $3.16 \mathrm{E}-02$ & $1.00 \mathrm{E}-03$ & $6.40 \mathrm{E}-03$ \\
\hline 12.6 & $3.98 \mathrm{E}-02$ & $1.58 \mathrm{E}-03$ & $4.04 \mathrm{E}-03$ \\
\hline 12.7 & $5.01 \mathrm{E}-02$ & $2.51 \mathrm{E}-03$ & $2.55 \mathrm{E}-03$ \\
\hline 12.8 & $6.31 \mathrm{E}-02$ & $3.98 \mathrm{E}-03$ & $1.61 \mathrm{E}-03$ \\
\hline 12.9 & $7.94 \mathrm{E}-02$ & $6.31 \mathrm{E}-03$ & $1.01 \mathrm{E}-03$ \\
\hline 13.0 & $1.00 \mathrm{E}-01$ & $1.00 \mathrm{E}-02$ & $6.40 \mathrm{E}-04$ \\
\hline 13.1 & $1.26 \mathrm{E}-01$ & $1.58 \mathrm{E}-02$ & $4.04 \mathrm{E}-04$ \\
\hline 13.2 & $1.58 \mathrm{E}-01$ & $2.51 \mathrm{E}-02$ & $2.55 \mathrm{E}-04$ \\
\hline 13.3 & $2.00 \mathrm{E}-01$ & $3.98 \mathrm{E}-02$ & $1.61 \mathrm{E}-04$ \\
\hline 13.4 & $2.51 \mathrm{E}-01$ & $6.31 \mathrm{E}-02$ & $1.01 \mathrm{E}-04$ \\
\hline 13.5 & $3.16 \mathrm{E}-01$ & $1.00 \mathrm{E}-01$ & $6.40 \mathrm{E}-05$ \\
\hline 13.6 & $3.98 \mathrm{E}-01$ & $1.58 \mathrm{E}-01$ & $4.04 \mathrm{E}-05$ \\
\hline 13.7 & $5.01 \mathrm{E}-01$ & $2.51 \mathrm{E}-01$ & $2.55 \mathrm{E}-05$ \\
\hline 13.8 & $6.31 \mathrm{E}-01$ & $3.98 \mathrm{E}-01$ & $1.61 \mathrm{E}-05$ \\
\hline 13.9 & $7.94 \mathrm{E}-01$ & $6.31 \mathrm{E}-01$ & $1.01 \mathrm{E}-05$ \\
\hline 14.0 & $1.00 \mathrm{E}+00$ & $1.00 \mathrm{E}+00$ & $6.40 \mathrm{E}-06$ \\
\hline
\end{tabular}




\section{REFERENCES}

[1] G. Yu, J. Gao, J. C. Hummelen, F. Wudl, A. J. Heeger, Science 1995, 270, 1789.

[2] M. Graetzel, in European Conference on Hybrid and Organic Solar Cells, Paris, 2006.

[3] N. Robertson, Angew. Chem. Int. Ed. 2006, 45, 2338.

[4] D. L. Talavera, G. Nofuentes, J. Aguilera, M. Fuentes, Renewable Sustainable Energy Rev. 2007, 11, 447.

[5] M. Graetzel, Nature 2001, 414, 338.

[6] L. B. Roberson, M. A. Poggia, J. Kowalik, G. P. Smestad, L. A. Bottomley, L. M. Tolberta, Coord. Chem. Rev. 2004, 248, 1491.

[7] L. L. Kazmerski, J. Electron. Spectrosc. Relat. Phenom. 2006, 150, 105.

[8] R. Swanson, in Proceedings of the 20th European PV Solar Energy Conference, Barcelona, 2004.

[9] G. P. Smestad, M. Graetzel, J. Chem. Educ. 1998, 75, 752.

[10] J. Hagen, W. Schaffrath, P. Otschik, R. Fink, A. Bacher, H.-W. Schmidt, D. Haarer, Synth. Met. 1997, 89, 215.

[11] M. Graetzel, J. Photochem. Photobiol., C Photochem. Rev. 2003, 4, 145.

[12] G. E. Tulloch, J. Photochem. Photobiol., A 2004, 164, 209.

[13] O. Kluth, B. Rech, L. Houben, S. Wieder, G. Schőpe, C. Beneking, H. Wagner, A. Lőffl, H. W. Schock, Thin Solid Films 1999, 351, 247.

[14] S. J. Tark, M. G. Kang, S. Park, J. H. Jang, J. C. Lee, W. M. Kim, J. S. Lee, D. Kim, Current Applied Physics 2009, 9, 1318.

[15] A. F. Nogueira, C. Longo, M.-A. De Paoli, Coord. Chem. Rev. 2004, 248, 1455.

[16] K. Hara, T. Horiguchi, T. Kinoshita, K. Sayama, H. Sugihara, H. Arakawa, Sol. Energy Mater. Sol. Cells 2000, 64, 115.

[17] M. Graetzel, Curr. Opin. Colloid Interface Sci. 1999, 4, 314.

[18] D. Gebeyehu, N. S. Sariciftcia, D. Vangeneugden, R. Kieboomsb, D. Vanderzandeb, F. Kienbergerc, H. Schindler, Synth. Met. 2001, 125, 279.

[19] D. Gebeyehu, C. J. Brabec, N. S. Sariciftci, Thin Solid Films 2002, 403-404, 271.

[20] K. Hara, T. Horiguchi, T. Kinoshitab, K. Sayama, H. Arakawa, Sol. Energy Mater. Sol. Cells 2001, 70151.

[21] L. H. Slooff, M. M. Wienk, J. M. Kroon, Thin Solid Films 2004, 451-452, 634.

[22] Y.-J. Lin, L. Wang, W.-Y. Chiu, Thin Solid Films 2006, 511-512, 199.

[23] P. Qin, X. Yang, R. Chen, L. Sun, J. Phys. Chem. C 2007, 111, 1853.

[24] B. Bowerman, V. Fthenakis, United States Department of Energy, New York, 2001.

[25] J. B. Baxter, E. S. Aydil, Sol. Energy Mater. Sol. Cells 2006, 90, 607.

[26] L. Bay, K. West, B. Winther-Jensen, T. Jacobsen, Sol. Energy Mater. Sol. Cells 2006, 90341.

[27] D. C. Olson, J. Pirisa, R. T. Collinsb, S. E. Shaheena, D. S. Ginleya, Thin Solid Films 2006, 496, 26.

[28] S. Deb, K, Sol. Energy Mater. Sol. Cells 2005, 88, 1.

[29] A. Du Pasquier, Electrochim. Acta 2007, 52, 7469.

[30] G. Boschloo, H. Lindström, E. Magnusson, A. Holmberg, A. Hagfeldt, J. Photochem. Photobiol., A 2002, 148, 11.

[31] C. Y. Jiang, X. W. Sun, K. W. Tan, G. Q. Lo, A. K. K. Kyaw, D. L. Kwong, Appl. Phys. Lett. 2008, 92, 143101.

[32] B. O'Regan, M. Graetzel, Nature 1991, 353, 737.

[33] F. Williams, A. J. Nozik, Nature 1984, 312, 21.

[34] W. Shockley, H. J. Queisser, Journal of Applied Physics 1961, 32, 510. 
[35] A. de Vos, Endoreversible Thermodynamics of Solar Energy Conversion, Oxford Science Publishers, Oxford, 1992.

[36] S. O. Kasap, Optoelectronics and Photonics: Principles and Practices, Prentice-Hall Inc., 2001.

[37] A. Harriman, Nature 1978, 276, 15.

[38] P. Wang, S. M. Zakeeruddin, J. E. Moser, M. K. Nazeeruddin, T. Sekiguchi, M. Graetzel, Nat. Mater. 2003, 2, 402.

[39] S. M. Zakeeruddin, M. K. Nazeeruddin, R. Humphry-Baker, P. Pechy, P. Quagliotto, C. Barolo, G. Viscardi, M. Graetzel, Langmuir 2002, 18, 952.

[40] D. Kuang, S. Ito, B. Wenger, C. Klein, J.-E. Moser, R. Humphry-Baker, S. M. Zakeeruddin, M. Graetzel, J. Am. Chem. Soc. 2006, 128, 4146.

[41] Y. Chi, P.-T. Chou, Chem. Soc. Rev. 2007, 36, 1421.

[42] O. Schwarz, D. van Loyen, S. Jockusch, N. J. Turro, H. Dürr, J. Photochem. Photobiol., A 2000, 132, 91 .

[43] F. Cecchet, A. M. Gioacchini, M. Marcaccio, F. Paolucci, S. Roffia, M. Alebbi, C. A. Bignozzi, J. Phys. Chem. B 2002, 106, 3926.

[44] P.-H. Xie, Y.-J. Hou, T.-X. Wei, B.-W. Zhang, Y. Cao, C.-H. Huang, Inorg. Chim. Acta 2000, 308, 73.

[45] F. Mazille, Z. Fei, D. Kuang, D. Zhao, S. M. Zakeeruddin, M. Graetzel, P. J. Dyson, Inorg. Chem. 2006, 45, 1585.

[46] Z. Fei, D. Kuang, D. Zhao, C. Klein, W. H. Ang, S. Zakeeruddin, M. Graetzel, P. J. Dyson, Inorg. Chem. 2006, 45, 10407.

[47] M. Spath, P. M. Sommeling, J. A. M. van Roosmalen, H. J. P. Smit, N. P. G. van der Burg, D. R. Mahieu, N. J. Bakker, J. M. Kroon, Progress iin PhotoVoltaics 2003, 11, 207.

[48] R. J. Ellingson, J. B. Asbury, S. Ferrere, A. N. Ghosh, T. Lian, A. J. Nozik, Phys. Chem. 1999, 21277.

[49] K. Tennakone, G. R. R. Kumara, I. R. M. Kottegoda, V. S. P. Perera, Chem. Commun. 1998, 10,3825 .

[50] T. Oekermann, T. Yoshidaa, H. Tadac, H. Minour, Thin Solid Films 2006, 511-512, 354.

[51] K. Sayama, H. Suguhara, H. Arakawa, Chem. Mater. 1998, 10, 3825.

[52] T. Hoshikawa, T. Ikebe, R. Kikuchi, K. Eguchi, Electrochim. Acta 2006, 515286.

[53] S. Ito, S. M. Zakeeruddin, R. Humphry-Baker, P. Liska, R. Charvet, P. Comte, M. K. Nazeeruddin, P. Péchy, M. Takata, H. Miura, S. Uchida, M. Graetzel, Adv. Mater. 2006, 28, 1202.

[54] M. Ni, M. K. H. Leung, D. Y. C. Leung, K. Sumathy, Sol. Energy Mater. Sol. Cells 2006, 90, 1331.

[55] K. D. Benkstein, N. Kopidakis, J. v. d. Lagemaat, A.J. Frank, J. Phys. Chem. B 2003, 107, 7759.

[56] Y. Saito, S. Kambe, T. Kitamura, Y. Wada, S. Yanagida, Sol. Energy Mater. Sol. Cells 2004, $83,1$.

[57] C. J. Barbe, F. Arendse, P. Comte, M. Jirousck, F. Lenzmann, V. Shklover, M. Graetzel, J. Am. Chem. Soc. 1997, 80, 3157.

[58] R. E. A. Dillon, D. F. Shriver, Chem. Mater. 1999, 11, 3296.

[59] M. Telford, Materials Today 2005, July/August, 15.

[60] Z. Kebede, S.-E. Lindquist, Sol. Energy Mater. Sol. Cells 1999, 57, 259.

[61] A. Fukui, R. Komiya, R. Yamanaka, A. Islam, L. Han, Sol. Energy Mater. Sol. Cells 2006, 90 649.

[62] H. Kusama, H. Arakawa, Sol. Energy Mater. Sol. Cells 2004, 81, 87.

[63] J. Wu, Z. Lan, J. Lin, M. Huang, P. Li, J. Power Sources 2007, 173, 585.

[64] H. Tributsch, Coord. Chem. Rev. 2004, 248, 1511.

[65] G. Oskam, B. V. Bergeron, G. J. Meyer, P. C. Searson, J. Phys. Chem. B 2001, 105, 6867. 
[66] H. Nusbaumer, J.-E. Moser, S. M. Zakeeruddin, M. K. Nazeeruddin, M. Graetzel, J. Phys. Chem. B 2001, 105, 10461.

[67] Q.-B. Meng, K. Takahashi, X.-T. Zhang, I. Sutanto, T. N. Rao, O. Sato, A. Fujishima, Langmuir 2003, 19, 3572.

[68] Y. Liu, A. Hagfeldt, X.-R. Xiao, S.-E. Lindquist, Sol. Energy Mater. Sol. Cells 1998, 55267.

[69] H. Wang, J. Bell, J. Desilvestro, M. Bertoz, G. Evans, J. Phys. Chem. C 2007, 111, 15125.

[70] K. Fredin, J. Nissfolk, G. Boschloo, A. Hagfeldt, J. Electroanal. Chem. 2007, 609, 55.

[71] C. Shi, S. Daia, K. Wang, X. Apn, L. Zeng, L. Hu, F. Konga, L. Guo, Electrochim. Acta 2005, 502597.

[72] H. Paulsson, L. Kloo, A. Hagfeldt, G. Boschloo, J. Electroanal. Chem. 2006, 586, 56.

[73] C. A. Kelly, F. Farzad, D. W. Thompson, J. M. Stipkala, G. J. Meyer, Langmuir 1999, 15, 7047.

[74] B. Enright, G. Redmond, D. Fitzmaurice, J. Phys. Chem. 1994, 98, 6195.

[75] P. Wang, S. M. Zakeeruddin, R. Humphry-Baker, M. Graetzel, Chem. Mater. 2004, 16, 2694.

[76] R. Kawano, H. Matsui, C. Matsuyama, A. Sato, M. A. B. H. Susan, N. Tanabe, M. Watanabe, J. Photochem. Photobiol., A 2004, 164, 87.

[77] P. Wang, S. M. Zakeeruddin, P. Comte, I. Exnar, M. Graetzel, J. Am. Chem. Soc. 2003, 125, 1166.

[78] W. Kubo, T. Kittamura, K. Nanabusa, Y. Wada, S. Yanagida, Chem. Commun. 2002, 247.

[79] E. Stathatos, P. Lianos, V. Jovanovski, B. Orel, J. Photochem. Photobiol., A 2005, 169, 57.

[80] P. Wang, S. M. Zakeeruddin, P. Comte, I. Exnar, M. Graetzel, J. Am. Chem. Soc. 2003, 125, 1166.

[81] M. Kosmulski, W. J. Janusz, J. Colloid Interface Sci. 2002, 242, 104.

[82] M. Gorlov, H. Pettersson, A. Hagfeldt, L. Kloo, Inorg. Chem. 2007, 463566.

[83] B. Orel, A. S. Vuk, V. Jovanovski, R. Jese, L. S. Perse, S. B. Hocevar, E. A. Hutton, B. Ogorevc, A. Jesih, Electrochem. Commun. 2005, 7, 692.

[84] D. Kuang, P. Wang, S. Ito, S. M. Zakeeruddin, M. Graetzel, J. Am. Chem. Soc. 2006, 128, 7732.

[85] M. Zistler, P. Wachter, P. Wasserscheid, D. Gerhard, A. Hinsch, R. Sastrawan, H. J. Gores, Electrochim. Acta 2006, 52, 161.

[86] R. Kawano, M. Watanabe, Chem. Commun. 2005, 2107.

[87] N. Yamanaka, R. Kawano, W. Kubo, T. Kitamura, Y. Wada, M. Watanabe, S. Yanagida, Chem. Commun. 2005740.

[88] A. J. Battacharyya, J. Maier, Adv. Mater. 2004, 16, 811.

[89] H. Wang, H. Li, B. Xue, Z. Wang, Q. Meng, L. Chen, J. Am. Chem. Soc. 2005, 127, 6394.

[90] H. An, B. Xue, D. Li, H. Li, Q. Meng, L. Guo, L. Chen, Electrochem. Commun. 2006, 8, 170.

[91] B. Xue, H. Wang, Y. Hu, H. Li, Z. Wang, Q. Meng, X. Huang, L. Chen, O. Sato, A. Fujishima, C. R. Chimie 2006, 9, 627.

[92] B. Xue, H. Wang, H. YongSheng, H. Li, Z. Wang, Q. Meng, X. Huang, O. Sato, L. Chen, A. Fujishima, Photochem. Photobiol. Sci 2004, 3, 918.

[93] M. Berginc, M. Hočevar, U. O. Krašovec, A. Hinsch, R. Sastrawan, M. Topič, Thin Solid Films 2008, 516, 4645.

[94] B.-F. Xue, H.-X. Wang, Y.-S. Hu, H. Li, Z.-X. Wang, Q.-B. Meng, X.-J. Huang, L.-Q. Chen, O. Sato, A. Fujishima, Chinese Phys. Lett. 2004, 21, 1828.

[95] H. Yang, Y.-F. Cheng, F.-Y. Li, Z.-G. Zhou, T. Yi, Chun-Hui, N.-Q. Jia, Chinese Phys. Lett. 2005, 22, 2116.

[96] A. Fujishima, X.-T. Zhang, Proc. Japan Acad. Ser. B 2005, 81, 33.

[97] K. Tennakone, G. R. R. A. Kumara, P M, A. R. Kumarasinghe, K. G. U. Wijayantha, Sirim, Semiconductor Science and Technology 1995, 10, 1689. 
[98] K. Tennakone, G. R. R. A. Kumara, P M, I. R. M. Kottegoda, K. G. U. Wijayantha, V. S. P. Perera, J. Phys. D: Appl. Phys. 1998, 31, 1492.

[99] B. Mahrov, G. Boschloo, A. Hagfeldt, H. Siegbahn, H. Rensmo, J. Phys. Chem. B 2004, 108, 11604.

[100] B. O'Regan, F. Lenzmann, J. Phys. Chem. B 2004, 108, 4342.

[101] U. Bach, D. Lupo, P. Compte, J. E. Moser, F. Weissortel, J. Salbeck, H. Spreitzer, M. Graetzel, Nature 1998, 395, 583.

[102] G. Kruger, R. Plass, M. Graetzel, H.-J. Matthieu, Appl. Phys. Lett. 2002, 81, 367.

[103] U. Bach, Y. Tachibana, J.-E. Moser, S. A. Haque, J. R. Durrant, M. Graetzel, D. R. Klug, J. Am. Chem. Soc. 1999, 121.

[104] H. J. Snaith, A. J. Moule, C. Klein, K. Meerholz, R. H. Friend, M. Graetzel, Nano Letters 2007, $248,3372$.

[105] W. J. Albery, Acc. Chem. Res. 1982, 15, 142.

[106] K. L. Stevenson, W. F. Erbelding, Solar Energy 1981, 27, 139.

[107] B. A. Parkinson, A. Heller, B. Miller, Appl. Phys. Lett. 1978, 33, 521.

[108] W. J. Albery, A. W. Foulds, K. J. Hall, A. R. Hillman, Nature 1979, 282, 793.

[109] W. J. Albery, M. D. Archer, Nature 1977, 270, 399.

[110] T. Borrmann, A. J. McFarlane, J. H. Johnston, Personal Communication 2004.

[111] J. H. Johnston, A. J. McFarlane, T. Borrmann (IPONZ Patent Application 537747) 2006.

[112] T. Borrmann, A. J. McFarlane, J. H. Johnston, in Smart Materials and Smart Technologies Workshop NZIC Conference, Chemistry at the Interface, Nelson New Zealand, 2003.

[113] T. Borrmann, A. James McFarlane, J. H. Johnston, A. Markwitz, N. Dytlewski, Surf. Interface Anal. 2005, 37, 695.

[114] T. Shedlovsky, A. Brown, J. Am. Chem. Soc. 1934, 56, 1066.

[115] T. Borrmann, J. H. Johnston, A. J. McFarlane, M. J. Richardson, S. O'Connor, Personal Communication 2007.

[116] M. Grätzel, Curr. Opin. Colloid Interface Sci. 1999, 4, 314.

[117] T. Borrmann, J. H. Johnston, A. J. McFarlane, K. J. MacKenzie, A. Nukui, Powder Diffraction 2008 23, 204.

[118] Y. Abe, T. Gunji, Prog. Polym. Sci. 2004, 29, 149.

[119] Y. Abe, R. Shimano, K. Arimitsu, T. Gunji, Journal of Polymer Science: Part A: Polymer Chemistry 2003, 41, 2250.

[120] J. Cihlář, Colloids and Surfaces A: Physicochemical and Engineering Aspects 1993, 70, 239.

[121] N. Miyata, K.-i. Fuke, Q. Chen, M. Kawashita, T. Kokubo, T. Nakamura, Biomaterials 2002, $23,3033$.

[122] A. Meiszterics, K. Sinkó, Colloids and Surfaces A: Physicochem. Eng. Aspects 2008, 319, 143.

[123] Newport, Oriel Product Training: Spectral Irradiance 2009.

[124] M. Law, L. Greene, J. Johnson, R. Saykally, P. Yang, Nat. Mater. 2005, 4, 455.

[125] T. Borrmann, J. H. Johnston, A. J. McFarlane, K. J. MacKenzie, A. Nukui, Personal Communication 2008.

[126] F. A. Miller, C. H. Wilkins, Anal. Chem. 1952, 24, 1253.

[127] G. Ayward, T. Findlay, SI Chemical Data, 5th ed., John Wiley \& Sons, Queensland, 2002.

[128] O. Chailapakul, M. Amatatongchai, P. Wilairat, K. Grudpan, D. Nacapricha, Talanta 2004, 64, 1253.

[129] American Society of Testing and Materials (ASTM) G173-03 reference spectra derived from Simple Model of the Atmospheric Radiation Transfer of Sunshine (SMARTS) v. 2.9.2

[130] D. R. Lide, CRC Handbook of Chemistry and Physics, 86th ed., CRC Press, Taylor \& Francis, New York, 2005-2006.

[131] W. R. Fawcett, Langmuir 1989, 5, 661.

[132] A. Usami, Chem. Phys. Lett. 1997, 277, 105. 
[133] S. Hore, C. Vetter, R. Kern, H. Smit, A. Hinsch, Sol. Energy Mater. Sol. Cells 2006, 90, 1176.

[134] C. Lv, A. Govindasamy, H. Tsuboi, M. Koyama, A. Endou, M. Kubo, C. A. D. Carpio, A. Miyamoto, http://www.sccj.net/event/nenkai/2006sp/program/abstract-pdf/2O06.pdf (Accessed 26 June 2009).

[135] Y. Marcus, S. Glikberg, Pure \& Appl. Chem. 1985, 57, 855.

[136] G. Engelhardt, D. Michel, High resolution solid-state NMR of silicates and zeolites, John Wiley \& Sons, Chichester, 1987.

[137] E. Lippmaa, M. Magi, A. Satnoson, G. Engelhardt, A.-R. Grimmer, J. Am. Chem. Soc. 1980, $102,4889$.

[138] A. Holleman, E. Wiberg, N. Wiberg, Inorganic Chemistry, Academic Press, San Diego, 2001.

[139] R. L. Mills, B. Dhandapani, M. Nansteel, J. He, T. Shannon, A. Echezuria, International Journal of Hydrogen Energy 2001, 26, 339.

[140] S. Davison, R. Newton, Conservation and Restoration of Glass, 2 ed., Butterworth-Heinemann, London, 2003.

[141] Clark-Products-Ltd, Safety Data Sheet: Sodium Silicate Solution, 2007.

[142] S. H. Kang, J.-Y. Kim, H. S. Kim, H.-D. Koh, J.-S. Lee, Y.-E. Sung, J. Photochem. Photobiol., A 2008, 200, 294. 